\title{
Ein mechanisch basiertes Dehnungsmodell für ermüdungsbeanspruchten Beton
}

Von der Fakultät für Bauingenieurwesen und Geodäsie der Gottfried Wilhelm Leibniz Universität Hannover

zur Erlangung des Grades

Doktor-Ingenieur

(Dr.-Ing.)

genehmigte Dissertation von

Dipl.-Ing. Christoph von der Haar 
Referent:

Korreferenten: $\quad$ Prof. Dr. phil. Dr. techn. Konrad Bergmeister

Prof. Dr.-Ing. Ludger Lohaus

Vorsitz:

Prof. Dr.-Ing. Steffen Marx

Prof. Dr.-Ing. Martin Achmus 


\section{Kurzfassung}

Beton weist unter zyklischen Lasten ein komplexes Verformungsverhalten auf. Dieses beeinflusst die Spannungsverteilung auf der Querschnittsebene und vermag sich positiv auf die Lebensdauer von Stahlbeton- und Spannbetonkonstruktionen auszuwirken. Das normative Ermüdungsnachweiskonzept prognostiziert die Lebensdauer von Betonbauteilen allein auf der Grundlage von Wöhlerlinien. Die Verformungsentwicklung des Betons und die Spannungsumlagerungen auf der Querschnittsebene finden keine Berücksichtigung. Die auftretenden Verformungsprozesse und -mechanismen sind allerdings bis heute auch nicht abschließend geklärt, sodass zurzeit keine Weiterentwicklung des Ermüdungsnachweiskonzeptes möglich ist.

In dieser Arbeit wird das Verformungsverhalten von ermüdungsbeanspruchtem Beton auf der Grundlage eines additiven Dehnungsmodells betrachtet. In dem Modell wird von der Hypothese ausgegangen, dass unter einer Ermüdungsbeanspruchung elastische, viskose, thermische und schädigungsinduzierte Dehnungen im Beton auftreten. Die einzelnen Dehnungsanteile entwickeln sich individuell in Abhängigkeit von der Zahl der Lastwechsel, der Versuchsdauer und den zyklischen Beanspruchungsparametern. Kernstück der Arbeit ist die Bestimmung eines kriechaffinen Beanspruchungsniveaus, um den viskosen Verformungsanteil in Abhängigkeit von der Ermüdungsbeanspruchung abschätzen zu können. Als kriechaffines Beanspruchungsniveau wird dasjenige Spannungsniveau bezeichnet, welches unter einer konstanten Druckbeanspruchung die gleichen viskosen Verformungen hervorruft wie unter der Ermüdungsbeanspruchung bei gleicher Beanspruchungsdauer. Das kriechaffine Beanspruchungsniveau wird auf der Grundlage einer rheologischen Modellvorstellung und für verschiedene Kriechtheorien abgeleitet und für unterschiedliche Mittelspannungen, Spannungsamplituden und Belastungsfrequenzen bestimmt.

Auf der Grundlage des kriechaffinen Beanspruchungsniveaus und bekannter Zusammenhänge für die elastischen und thermischen Dehnungen können drei der vier Dehnungsanteile des additiven Dehnungsmodells beschrieben werden, sodass letztlich auch der schädigungsinduzierte Dehnungsanteil identifizierbar wird. Die gemessenen Dehnungen ermüdungsbeanspruchter Betonproben werden entsprechend der additiven Modellvorstellung zerlegt und analysiert. Es zeigt sich, dass sich der schädigungsinduzierte Dehnungsanteil in Abhängigkeit der logarithmierten Bruchlastwechselzahl linear entwickelt. Dementsprechend spiegelt sich der lineare Zusammenhang zwischen dem Oberspannungsniveau und der logarithmierten Bruchlastwechselzahl, wie er in den Wöhlerlinien für Beton sichtbar ist, auch im Dehnungsverhalten des Betons wider. Andererseits nehmen die Bruchdehnungen ermüdungsbeanspruchter Betonproben einen konstanten Wert an, wenn die gemessenen Verformungen um die thermischen und viskosen Dehnungsanteile korrigiert werden.

Schlagwörter: Beton, Ermüdungsbeanspruchung, Dehnungsentwicklung, additives Dehnungsmodell, kriechaffines Spannungsniveau 



\begin{abstract}
Concrete exhibits complex deformation behaviour under cyclic loads. This results in considerable stress redistributions in the cross-sections, which could have a positive effect on the service life of concrete constructions. The normative fatigue design concept predicts the service life based on $S-N$ curves only; cross-sectional deformations and stress redistributions are neglected. However, the occurring deformation processes and mechanisms are not yet fully understood, which makes it very difficult to develop the existing fatigue design concept further.

In this dissertation, the deformation behaviour of concrete is considered according to an additive strain model. The model assumes that under cyclic loads elastic, viscous, thermal, and damageinduced strains occur in concrete. The strain components develop independently of each other as a function of the number of load cycles, the test duration, and stress level. This dissertation focuses on the determination of a creep-relevant stress level to estimate the viscous deformation component. The creep-relevant stress level is the stress level which causes the same viscous deformations under constant loads as under cyclic loads for the same test duration. The creeprelevant stress level is derived from a rheological model and for different creep theories and is determined for different stress levels and load frequencies.

Based on the creep-relevant stress levels and known relationships for elastic and thermal strains, three of the four strain components of the additive strain model can be described. Thus, the damage-induced strain component can also be identified. Experimentally measured fatigue strains of concrete specimens are separated into the strain components assumed by the additive strain model. The components are evaluated and analysed individually. It was found that the damageinduced strain component develops linearly as a function of the logarithmic load cycles to failure in the examined cycle range. Accordingly, the linear relationship between the maximum compressive stress level and the logarithmic load cycles to failure is reflected in the strain behaviour, which is visible in the $S-N$ curves for concrete. On the other hand, the ultimate fatigue strain seems to be constant if the measured deformations are corrected by the thermal and viscous strains.
\end{abstract}

Keywords: concrete, fatigue loading, strain development, additive strain model, creep-relevant load level 



\section{Vorwort}

Die vorliegende Arbeit entstand während meiner Tätigkeit als wissenschaftlicher Mitarbeiter am Institut für Massivbau der Leibniz Universität Hannover.

Mein besonderer Dank gilt meinem Doktorvater, Herrn Prof. Dr.-Ing. Steffen Marx, für die Anregungen und die wissenschaftliche Begleitung meiner Arbeit.

Herrn Prof. Dr. phil. Dr. techn. Konrad Bergmeister und Herrn Prof. Dr.-Ing. Ludger Lohaus danke ich für das entgegengebrachte wissenschaftliche Interesse an meiner Arbeit und die Übernahme der Korreferate. Herrn Prof. Dr.-Ing. Martin Achmus danke ich für die Übernahme des Prüfungsvorsitzes.

Des Weiteren möchte ich mich bei all meinen Kollegen und wissenschaftlichen Hilfskräften herzlich bedanken die mir stets mit Rat und Tat geholfen haben, diese Arbeit zu vollenden.

Mein größter Dank gilt meiner Familie und meiner Frau Miriam für die jahrelange Unterstützung und das entgegengebrachte Verständnis. 



\section{Inhaltsverzeichnis}

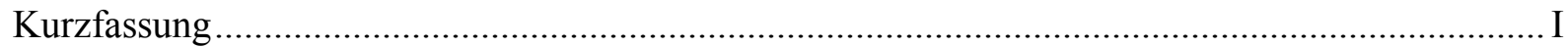

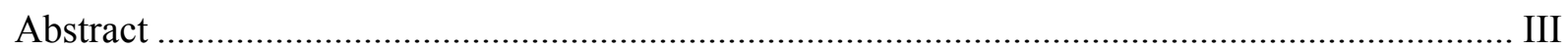

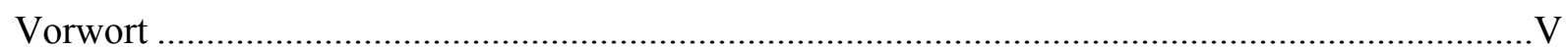

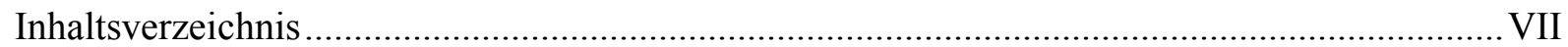

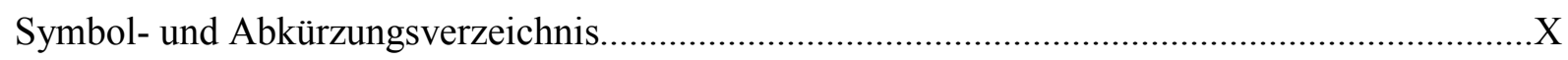

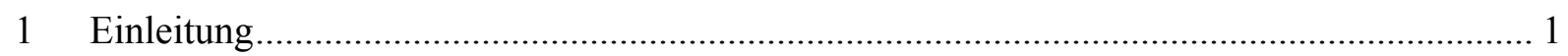

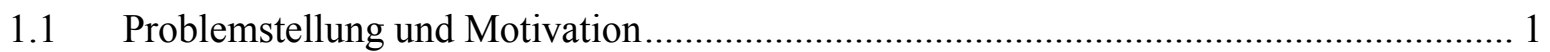

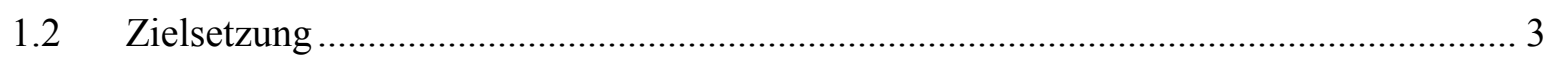

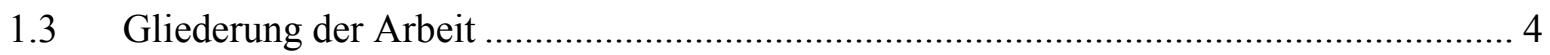

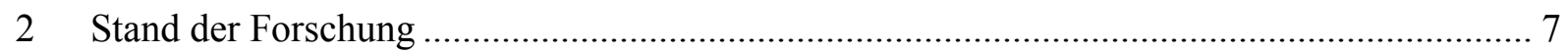

2.1 Materialverhalten unter monoton steigender Beanspruchung........................................ 7

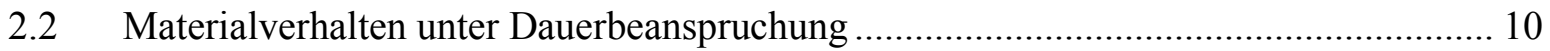

2.2.1 Phänomenologisches Materialverhalten .......................................................... 10

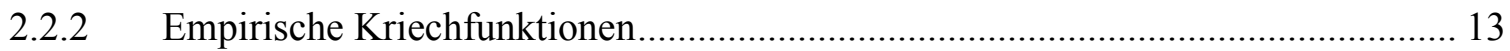

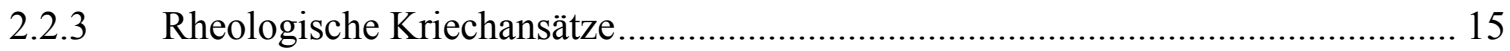

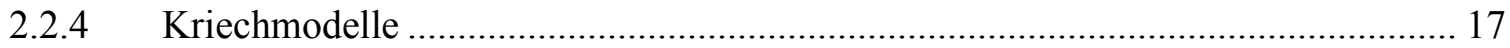

2.3 Materialverhalten unter zyklischer, verformungsgeregelter Beanspruchung ................ 23

2.4 Materialverhalten unter zyklischer, kraftgeregelter Beanspruchung ............................. 25

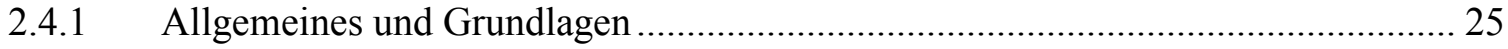

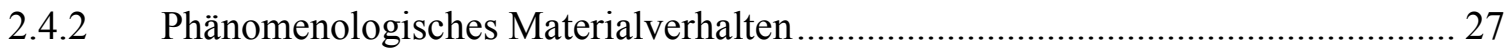

2.5 Energetische Betrachtung der Schädigungsprozesse im Beton...................................... 37

2.6 Dehnungsmodelle für ermüdungsbeanspruchten Beton.............................................. 39

2.6.1 Modelle basierend auf dem Envelope-Konzept.................................................. 39 
2.6.2 Energetisches Ermüdungsschädigungsmodell................................................ 40

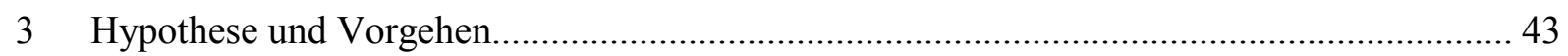

3.1 Einordnung der Arbeit in den Kontext der Literaturstudie ......................................... 43

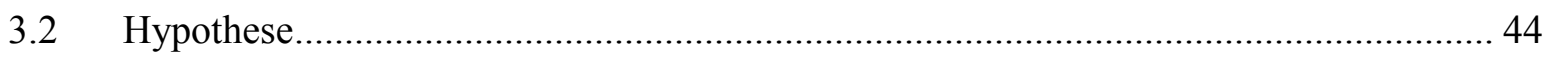

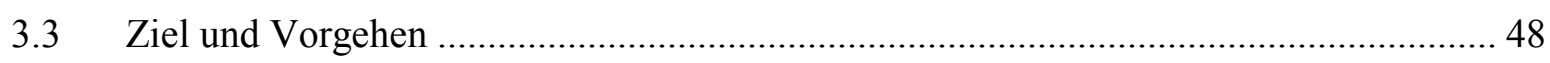

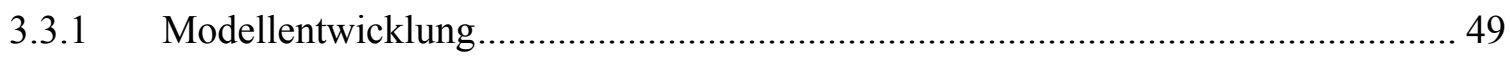

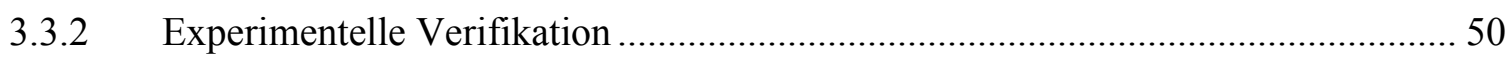

4 Modellbildung und Ableitung kriechaffiner Beanspruchungsniveaus .................................. 53

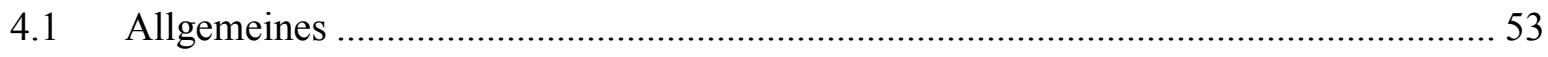

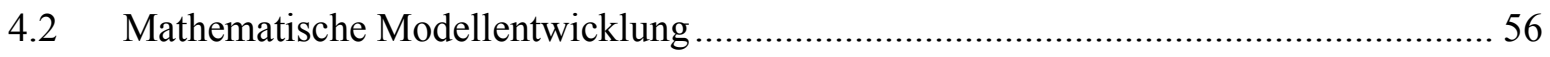

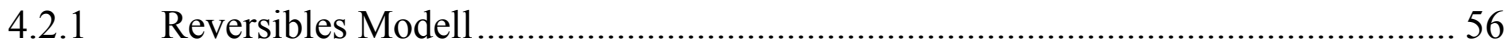

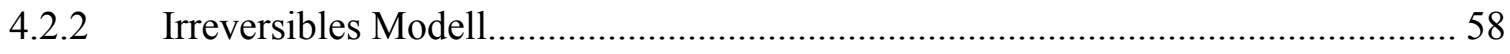

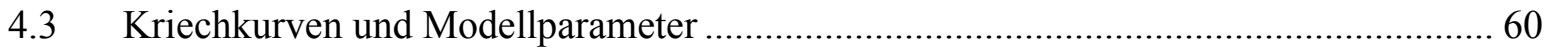

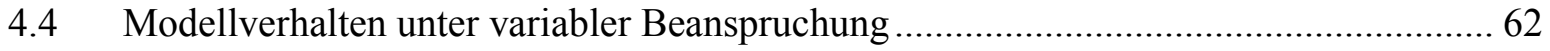

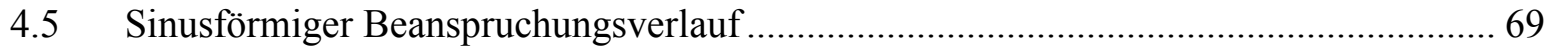

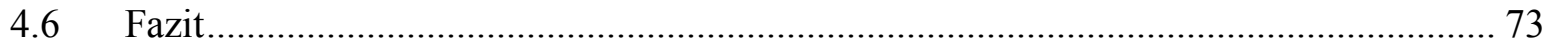

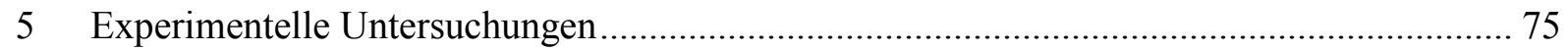

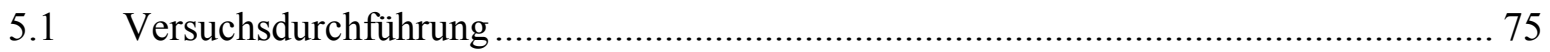

5.1.1 Betoneigenschaften und Probekörperherstellung ................................................. 75

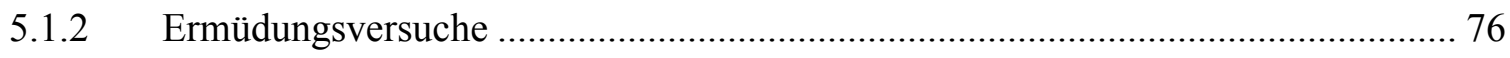

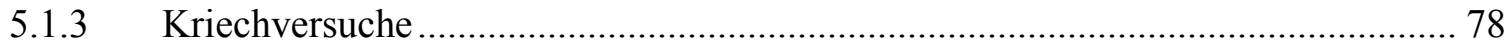

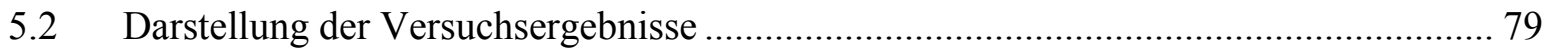

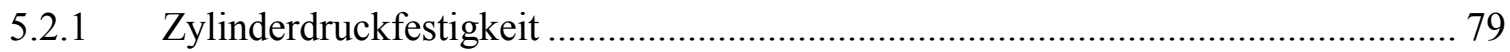

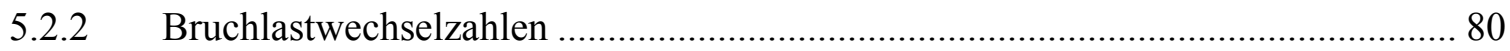

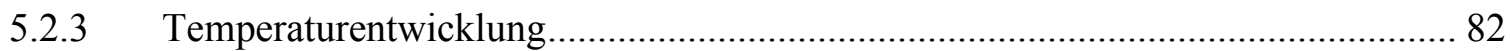

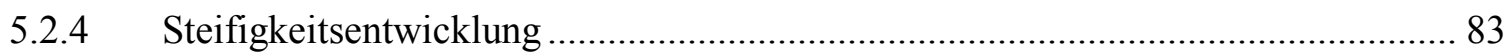




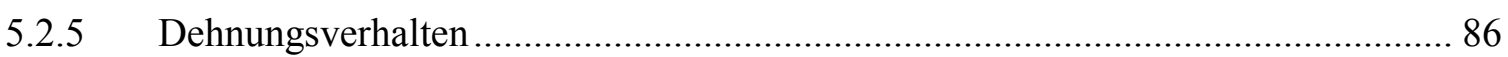

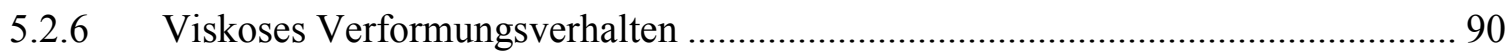

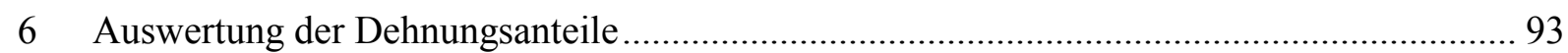

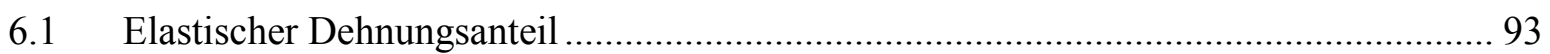

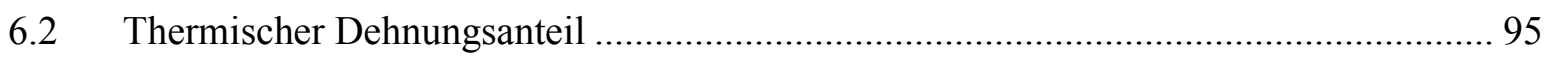

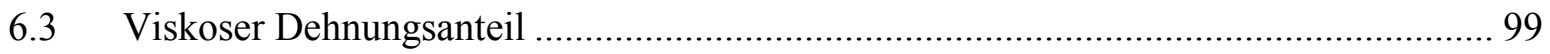

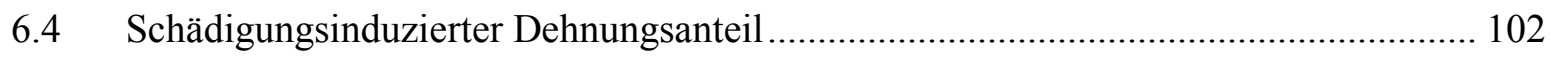

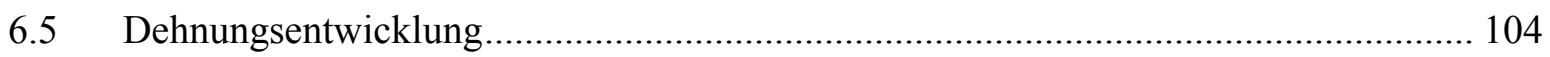

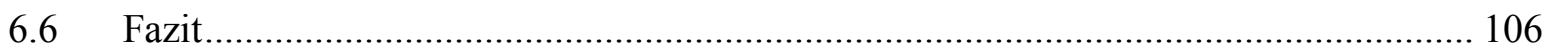

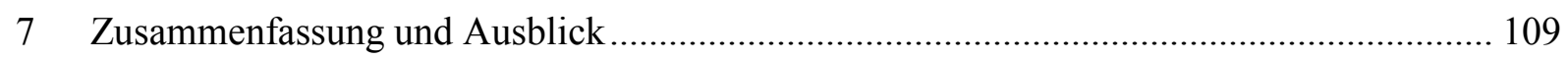

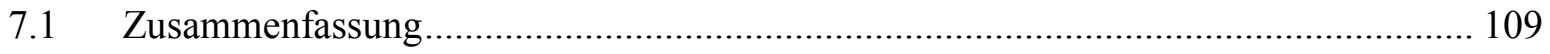

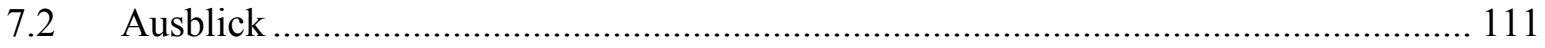

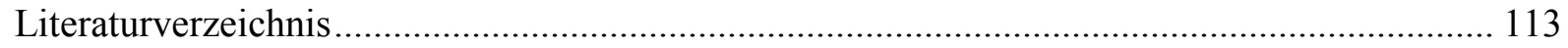

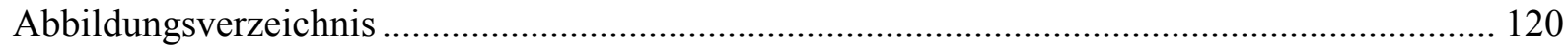

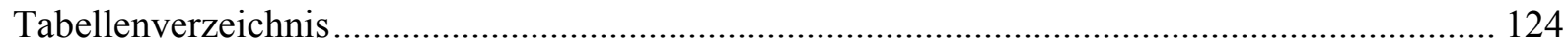

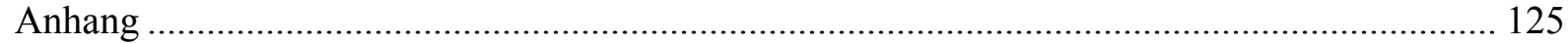




\section{Symbol- und Abkürzungsverzeichnis}

\section{Griechische Buchstaben}

$\varepsilon \quad$ Dehnung / Stauchung

$\varepsilon_{c} \quad$ Betondehnung bei Erreichen der Betondruckfestigkeit $f_{c}$ bei monoton steigender Beanspruchung

$\varepsilon_{c r} \quad$ Viskose Dehnung des Betons

$\varepsilon_{d} \quad$ Schädigungsinduzierte Dehnung des Betons im Ermüdungsversuch

$\varepsilon_{e l} \quad$ Elastische Dehnung des Betons

$\varepsilon_{f} \quad$ Irreversible Fließdehnung des Betons

$\varepsilon_{f a t} \quad$ Messbare Dehnung des Betons im Ermüdungsversuch

$\varepsilon_{o} \quad$ Dehnungsverlauf auf dem Oberspannungsniveau des Ermüdungsversuchs

$\varepsilon_{t} \quad$ Temperaturdehnung

$\varepsilon_{u} \quad$ Dehnungsverlauf auf dem Unterspannungsniveau des Ermüdungsversuchs

$\varepsilon_{v} \quad$ Verzögert-elastische Dehnung des Betons

$\bar{\varepsilon}_{o} \quad$ Steigung der Dehnungsentwicklung in der zweiten Phase auf dem

Oberspannungsniveau des Ermüdungsversuchs

$\bar{\varepsilon}_{u}^{-I I} \quad$ Steigung der Dehnungsentwicklung in der zweiten Phase auf dem

Unterspannungsniveau des Ermüdungsversuchs

$\varepsilon^{F} \quad$ Dehnung zum Zeitpunkt des Probekörperversagens im Ermüdungsversuch

$\eta, \eta_{d}, \eta_{v}, \eta_{f}$ Dämpfersteifigkeiten

$v_{c} \quad$ Querdehnungsverhältnis des Betons

$\sigma \quad$ Spannung

$\sigma_{a} \quad$ Spannungsamplitude im Ermüdungsversuch

$\sigma_{c r} \quad$ Spannung im Kriechversuch

$\sigma_{m} \quad$ Mittelwert der Spannungen im Ermüdungsversuch

$\sigma_{o} \quad$ Betragsmäßig größter Wert der Spannungen im Ermüdungsversuch

$\sigma_{u} \quad$ Betragsmäßig kleinster Wert der Spannungen im Ermüdungsversuch

$\Delta \sigma \quad$ Spannungsschwingbreite im Ermüdungsversuch

$\varphi \quad$ Kriechzahl

$\varphi_{\infty} \quad$ Endkriechzahl

$\psi \quad$ Phasenwinkel der zyklischen Beanspruchung

\section{Große lateinische Buchstaben}
C Konstante
E Elastizitätsmodul / Federsteifigkeit
$E_{c} \quad$ Elastizitätsmodul des Betons 
$E_{s} \quad$ Elastizitätsmodul ermittelt als Sekantenmodul für den Entlastungsast bei zyklischer Beanspruchung

$J \quad$ Kriech- oder Komplianzfunktion

$N \quad$ Anzahl aufgebrachter Lastwechsel

$N_{f} \quad$ Ertragbare Lastwechselzahl bis zum Bruch des Probekörpers (Bruchlastwechselzahl)

$P \quad$ Versagenswahrscheinlichkeit

$R \quad$ Verhältniswert aus Ober- und Unterspannung im Ermüdungsversuch

$R^{2} \quad$ Bestimmtheitsmaß

$S_{c r} \quad$ Auf die Betondruckfestigkeit $f_{c}$ bezogenes kriechaffines Spannungsniveau $\sigma_{c r}$ im Ermüdungsversuch

$S_{m} \quad$ Auf die Betondruckfestigkeit $f_{c}$ bezogene Mittelspannung $\sigma_{m}$ im Ermüdungsversuch

$S_{o} \quad$ Auf die Betondruckfestigkeit $f_{c}$ bezogene Oberspannung $\sigma_{o}$ im Ermüdungsversuch

$S_{u} \quad$ Auf die Betondruckfestigkeit $f_{c}$ bezogene Unterspannung $\sigma_{u}$ im Ermüdungsversuch

$\Delta S \quad$ Auf die Betondruckfestigkeit $f_{c}$ bezogene Spannungsschwingbreite $\Delta \sigma \mathrm{im}$

Ermüdungsversuch

$T \quad$ Temperatur

$\Delta T \quad$ Temperaturänderung

\section{Kleine lateinische Buchstaben}

$a, b, m, n \quad$ Konstanten

$f_{c} \quad$ Einaxiale Druckfestigkeit des Betons

$f_{c, \text { cube,28d }} \quad$ Einaxiale Würfeldruckfestigkeit des Betons im Alter von 28 Tagen

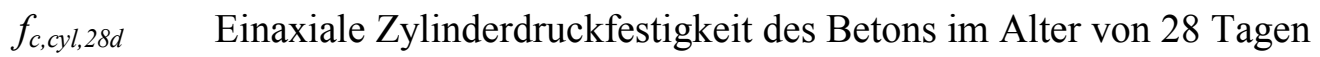

$f_{p} \quad$ Belastungsfrequenz im Ermüdungsversuch

$s \quad$ Standardabweichung einer Stichprobe

$t \quad$ Zeit

$t_{0} \quad$ Betonalter bei Belastungsbeginn

\section{Abkürzungen}

BLZ Bruchlastwechselzahl

LW Lastwechsel

w/z-Wert Wasser-Zementwert 



\section{$1 \quad$ Einleitung}

\subsection{Problemstellung und Motivation}

Stetige Weiterentwicklungen im Bereich der Betontechnologie ermöglichen die Realisierung immer schlankerer und filigranerer Betonkonstruktionen. Heutige Hochleistungsbetone erreichen die Festigkeitswerte ferritischer Werkstoffe bei zunehmend verbesserten Verarbeitungseigenschaften. Hierdurch kann der Beton in Bereichen eingesetzt werden, die bislang dem Stahl vorbehalten waren. Die Anwendung des Betons als reiner Massenbaustoff wandelt sich zum effizient eingesetzten Hochleistungswerkstoff. Dieser Trend hat zur Folge, dass die zyklischen Beanspruchungen bzw. Ermüdungsbeanspruchungen im Beton an Bedeutung gewinnen und einen zunehmenden Einfluss auf das Design der Konstruktion nehmen.

Zyklische Beanspruchungen rufen im Beton, wie auch in anderen Werkstoffen, Deformationsund Schädigungsprozesse hervor, die zum Versagen des Betonkörpers oder eines ganzen Bauteils führen können. Dieser Versagenszustand kann selbst bei Beanspruchungszuständen weit unterhalb der Materialfestigkeitsgrenze hervorgerufen werden. Um die Widerstandsfähigkeit des Betons gegenüber zyklischen Beanspruchungen zu quantifizieren, werden Ermüdungsversuche durchgeführt. Die Prüflast oszilliert im Ermüdungsversuch zwischen vorgegebenen Ober- und Unterlasten, bis der Probekörper versagt. Die ertragbaren Lastwechselzahlen bzw. Bruchlastwechselzahlen werden in Form von Wöhlerkurven dargestellt, die dann zur Bemessung ermüdungsbeanspruchter Betonbauwerke herangezogen werden können.

Der Ermüdungsnachweis für Beton kann entsprechend einem dreistufigen Nachweiskonzept geführt werden (Model Code 2010). Die Anforderungen und die unterstellte Genauigkeit des Nachweises steigen mit jeder Stufe. Auf der ersten Stufe darf die maximale, ermüdungsrelevante Bauteilspannung eine Grenzspannung, abgeleitet aus den Wöhlerkurven für $10^{8}$ Lastwechsel, nicht überschreiten. Bei Nichteinhaltung ist auf der zweiten Stufe die zu erwartende Lastwechselzahl des Bauteils abzuschätzen. Mithilfe dieser ist eine zulässige Spannung anhand der Wöhlerkurven zu ermitteln. Letztere ist der maximalen, ermüdungsrelevanten Bauteilspannung gegenüberzustellen. Auf der dritten Stufe darf der Nachweis auf der Grundlage der linearen Schädigungsakkumulationshypothese nach (Palmgren, 1924) und (Miner, 1945) erfolgen. Die Schädigungshypothese, ursprünglich entwickelt für die Bemessung von Stahlkugellagern, geht davon aus, dass jede zyklische Beanspruchung eine inkrementelle Schädigung im Bauteil erzeugt und diese sich bis zum Bruch linear akkumulieren. Der inkrementelle Schädigungswert je Lastzyklus wird anhand der Wöhlerkurven abgeschätzt. Das Ergebnis des Ermüdungsnachweises ist somit maßgeblich vom Verlauf der Wöhlerkurven abhängig. Um diesen möglichst genau festzulegen, wurden in der Vergangenheit Ermüdungsversuche an Betonen unterschiedlicher Festigkeiten, Zusammensetzungen und Lagerungsbedingungen sowie unter variierenden Prüf- und Belastungsbedingungen durchgeführt 
(Marx et al., 2013). Zentrales Motiv der meisten in der Fachliteratur veröffentlichten Versuchsergebnisse ist demzufolge die Darstellung der ertragbaren Lastwechselzahlen unter den vorgenannten Versuchs- und Probekörpervariationen. Sehr wenige beschäftigten sich mit der Klärung der Versagensmechanismen, der Schädigungsakkumulation, der Verformungsentwicklung und der Materialdegradation vor dem makroskopischen Versagen. Dementsprechend wurden auch nur bei wenigen Untersuchungen über die Lastwechselzahl hinausgehende Messdaten, wie die Betonstauchung oder die Steifigkeitsentwicklung, erfasst bzw. publiziert.

Das „reale“ Tragverhalten eines ermüdungsbeanspruchten Betonbauteils kann mit dem vorgestellten Bemessungskonzept nicht abgebildet werden. Die Ermüdungsbeanspruchung stellt sich auf Bauteilebene meist in Form einer zyklischen Biegebeanspruchung dar. Auf Querschnittsebene resultieren aus der zyklischen Biegebeanspruchung Spannungsschwingbreiten, die an den Querschnittsrändern größer sind als im Querschnittskern. Größere Spannungsschwingbreiten führen zu einer schnelleren Materialdegradation und Verformungsentwicklung im Beton als kleinere Spannungsschwingbreiten. Infolgedessen lagern sich die Spannungen mit zunehmender Lastwechselzahl aus den Randbereichen in die inneren Bereiche des Querschnitts um. Von diesen Spannungsumlagerungen wird erwartet, dass sie sich positiv auf die ertragbare Lastwechselzahl von dynamisch beanspruchten Betonbauwerken auswirken.

Um diese Spannungsumlagerungen abbilden zu können, sind Modelle erforderlich, die das Dehnungsverhalten und die Materialdegradation des zyklisch beanspruchten Betons prognostizieren. In der Fachliteratur sind jedoch nur wenige Modelle veröffentlicht, die dieses vermögen. Darüber hinaus unterliegen diese Modelle groben Vereinfachungen oder sind nicht ausreichend verifiziert. Das übergeordnete Ziel der veröffentlichten Modelle ist, die Dehnungsentwicklung bzw. die Bruchdehnung des Betons unter monoton steigender Beanspruchung mit dem Deformationsverhalten im Ermüdungsversuch ins Verhältnis zu setzen. Während die Versuchsdauer im Druckversuch bei monoton steigender Beanspruchung meist nur wenige Minuten beträgt, kann diese im Ermüdungsversuch mehrere Stunden bis Tage in Anspruch nehmen. Dabei werden die von der Belastungsdauer abhängigen viskosen Verformungsanteile des Betons nicht gesondert betrachtet. Gleichzeitig ist das Verformungsverhalten des Betons unter monoton steigender Beanspruchung auch wegen dem viskosen Verformungsanteil von der Belastungsgeschwindigkeit signifikant abhängig.

Während die Belastungsgeschwindigkeit die Verformungsentwicklung unter monoton steigender Beanspruchung beeinflusst, sind die Verformungen im Kriechversuch vom Druckspannungsniveau und der Belastungsdauer, neben weiteren Parametern wie Feuchte, Betonalter und Umgebungstemperatur, abhängig. Demgegenüber sind der Ermüdungsversuch und dessen Verformungen durch Mittelspannungsniveau, Spannungsschwingbreite, Belastungsfrequenz und Lastwechselzahl charakterisiert. Tendiert die Spannungsschwingbreite des Ermüdungsversuchs aber gegen null, gleicht der Beanspruchungsverlauf des Ermüdungsversuchs dem des Kriechversuchs. Andererseits entspricht der erste Belastungszyklus des Ermüdungsversuchs dem Materialverhalten des Betons unter monoton steigender Beanspruchung. Die Dehnungen im Druckversuch unter monoton steigender 
Druckbeanspruchung setzen sich zusammen aus elastischen und schädigungsinduzierten Verformungen und zum geringen Teil aus viskosen Verformungen. Demgegenüber treten im Kriechversuch, abgesehen von Schwindverformungen, vornehmlich elastische und viskose Verformungen auf, schädigungsinduzierte Verformungen spielen eine untergeordnete Rolle. Im Ermüdungsversuch sind, in Abhängigkeit vom Beanspruchungsniveau und der Versuchslaufzeit, alle drei Verformungsanteile in unterschiedlicher Größe zu erwarten. Dementsprechend muss in einem Verformungsmodell für ermüdungsbeanspruchten Beton zwischen elastischen, schädigungsinduzierten und viskosen Verformungsanteilen unterschieden werden, die sich spannungsabhängig, lastwechselzahlabhängig und zeitabhängig entwickeln.

Die Einwirkungen von dynamisch beanspruchten Betonbauwerken liegen im Bereich von bis zu $10^{9}$ Lastwechseln (Grünberg \& Göhlmann, 2013). Um das Ermüdungsverhalten von Beton innerhalb vertretbarer Prüfzeiten zu bestimmen, müssen Ermüdungsversuche bei erhöhten Spannungsschwingbreiten und/oder Belastungsfrequenzen durchgeführt werden, um das Versagen des Probekörpers im Versuch zu erzielen. Hieraus ergeben sich zusätzliche Prüfeinflüsse, wie eine Erwärmung der Betonproben während der Versuchsdurchführung. In der Literatur sind Probekörpererwärmungen auf bis zu $100{ }^{\circ} \mathrm{C}$ dokumentiert (Elsmeier et al., 2016). Es resultieren Temperaturdehnungen, die sich mit den elastischen, schädigungsinduzierten und viskosen Verformungen überlagern.

Derzeit fehlt das grundlegende Wissen, das Verformungsverhalten eines ermüdungsbeanspruchten Betonkörpers hinsichtlich der elastischen, schädigungsinduzierten, viskosen und thermischen Verformungsanteile im Ermüdungsversuch zu beschreiben. Dabei ermöglicht erst die separate Betrachtung und Interpretation der Verformungsanteile ein vertieftes, mechanismen-orientiertes Verständnis für die im Beton stattfindenden Verformungs- und Schädigungsprozesse. Allein auf dieser Basis können die auftretenden Deformationsprozesse modellmäßig erfasst und mit dem Verformungsverhalten unter monoton steigender Beanspruchung ins Verhältnis gesetzt werden.

\subsection{Zielsetzung}

Das Ziel dieser Arbeit ist, das viskose Verformungsverhalten von ermüdungsbeanspruchtem Beton in Abhängigkeit von den Belastungsparametern des Ermüdungsversuchs zu charakterisieren, um damit einen Beitrag zur Entwicklung von Dehnungsmodellen für ermüdungsbeanspruchten Beton zu liefern. Dabei spielt die Bestimmung des kriechaffinen Beanspruchungsniveaus eine maßgebende Rolle. Zur Erläuterung soll zunächst gedanklich von einem Ermüdungsversuch mit einer infinitesimal kleinen Spannungsschwingbreite ausgegangen werden. In diesem Fall gleicht der Beanspruchungsverlauf des Ermüdungsversuchs dem des Kriechversuchs. Das Beanspruchungsniveau, das im Kriechversuch die gleichen viskosen Verformungen hervorruft wie der Ermüdungsversuch, bei gleichen Umgebungsbedingungen und der gleichen Versuchsdauer, wird im Rahmen dieser Arbeit als das kriechaffine Spannungsniveau bezeichnet. Damit entspricht das Mittelspannungsniveau dem kriechaffinen Spannungsniveau. Bei Spannungsamplituden ungleich null wird erwartet, dass das kriechaffine Spannungsniveau 
gegenüber dem Mittelspannungsniveau zunimmt. Weiterhin wird untersucht, wie dieser Prozess vom Rückkriechen des Betons in den Entlastungsphasen des zyklischen Beanspruchungsverlaufs überlagert und von der Belastungsfrequenz beeinflusst wird. Das kriechaffine Spannungsniveau wird in dieser Arbeit auf der Grundlage rheologischer Modellvorstellungen abgeleitet. In diesem Rahmen wird der Einfluss der Spannungsamplitude, des Mittelspannungsniveaus, der Belastungsfrequenz sowie der Einfluss unterschiedlicher Kriechtheorien auf das kriechaffine Spannungsniveau untersucht.

Im Weiteren befasst sich die Arbeit mit den unterschiedlichen Verformungsanteilen von Betonprobekörpern im Ermüdungsversuch. Es wird von der Hypothese ausgegangen, dass sich die Gesamtverformungen aus elastischen, schädigungsinduzierten, viskosen und thermischen Verformungen zusammensetzen. Während der schädigungsinduzierte Dehnungsanteil aus der Beanspruchungsänderung je Lastwechsel resultiert, sind die viskosen Verformungen eine Folge der kontinuierlichen Wirkung der zyklischen Beanspruchung. Es wird von einer additiven Modellvorstellung ausgegangen. Die einzelnen Verformungsanteile werden durch die Spannungsamplitude und Mittelspannung, durch die Zahl der einwirkenden Lastwechsel, durch die Versuchsdauer und durch die Belastungsfrequenz individuell beeinflusst. In diesem Zusammenhang werden Ermüdungsversuche an zylindrischen Betonproben durchgeführt. Die im Experiment aufgezeichneten Dehnungsverläufe werden in die genannten Verformungsanteile aufgespalten. Hierzu wird auf das ermittelte kriechaffine Beanspruchungsniveau sowie auf bestehende Zusammenhänge für die elastischen und thermischen Dehnungen zurückgegriffen. Somit können drei der vier Dehnungsanteile der additiven Modellvorstellung beschrieben werden, wodurch auch der schädigungsinduzierte Dehnungsanteil ermittelt werden kann. Die Verformungsanteile werden analysiert und hinsichtlich ihrer Gewichtung an der Gesamtverformung quantifiziert.

Die Erkenntnisse dieser Arbeit sollen einen Beitrag zur Identifizierung und Beschreibung der einzelnen, während eines Ermüdungsversuchs stattfindenden Verformungsprozesse liefern. Auf der Grundlage des additiven Dehnungsmodells und des kriechaffinen Beanspruchungsniveaus können die Dehnungsanteile ermüdungsbeanspruchter Betonprobekörper gegenübergestellt und verglichen werden, selbst wenn die zugehörigen Ermüdungsversuche auf unterschiedlichen Beanspruchungsniveaus und Belastungsfrequenzen durchgeführt werden und damit unterschiedlich lange dauern. Langfristig gesehen können auf dieser Basis Dehnungsmodelle für ermüdungsbeanspruchten Beton und dehnungsbasierte Versagenskriterien entwickelt werden, um die Spannungsumlagerungen und das Ermüdungsverhalten von zyklisch beanspruchten Stahlbeton- und Spannbetonbauteilen unter Biegebeanspruchung vorhersagen zu können.

\subsection{Gliederung der Arbeit}

Im Anschluss an die Einleitung werden im Kapitel 2 die in der Literatur dokumentierten Erkenntnisse zum Materialverhalten von Beton unter monoton steigender Beanspruchung, unter zyklisch-verformungsgeregelter und zyklisch-kraftgeregelter Beanspruchung sowie unter 
Dauerbeanspruchung behandelt. Im Fokus steht die Beschreibung und Charakterisierung der auftretenden Verformungen unter den genannten Beanspruchungsarten. Des Weiteren werden in der Literatur bekannte Dehnungsmodelle für dauerhaft und zyklisch beanspruchten Beton dargestellt und die Vor- und Nachteile der einzelnen Modelle diskutiert.

In Kapitel 3 werden das additive Dehnungsmodell und die Ansätze zur Trennung der einzelnen Dehnungsanteile von ermüdungsbeanspruchtem Beton entwickelt. Darauf folgend wird das Vorgehen zur Bestimmung des kriechaffinen Beanspruchungsniveaus vorgestellt. Abschließend werden Anforderungen an die durchzuführenden experimentellen Untersuchungen definiert, um den Einfluss der Beanspruchungshöhe, der Beanspruchungsfrequenz und der Versuchsdauer auf die verschiedenen Verformungsanteile signifikant darstellen zu können.

Das Kapitel 4 beinhaltet die mathematische Bestimmung des kriechaffinen Beanspruchungsniveaus. Der notwendige Formelapparat wird unter Ansatz unterschiedlicher Kriechtheorien abgeleitet und programmtechnisch umgesetzt. Hierzu wird auf eine rheologische Modellvorstellung zurückgegriffen. Die kriechaffinen Beanspruchungsniveaus werden in Abhängigkeit des Mittelspannungsniveaus, der Spannungsamplitude und der Belastungsfrequenz berechnet und diskutiert.

Das Kapitel 5 beinhaltet eine allgemeine Darstellung der experimentellen Untersuchungen und eine grundlegende Versuchsdatenauswertung. Es werden Versuche unter monoton steigender Beanspruchung, Dauerbeanspruchung und Ermüdungsbeanspruchung durchgeführt. Die Versuchsdatenauswertung erfolgt $u$. a. hinsichtlich der erreichten Bruchlastwechselzahlen sowie der Probekörpererwärmung im Ermüdungsversuch. Im Weiteren werden die Ergebnisse der Versuche unter monoton steigender Beanspruchung und Dauerbeanspruchung vorgestellt.

Demgegenüber erfolgt im Kapitel 6 die Separierung der unterschiedlichen Dehnungsanteile entsprechend der additiven Modellvorstellung. Die einzelnen Dehnungsanteile werden in Abhängigkeit der untersuchten Beanspruchungsniveaus und Belastungsfrequenzen präsentiert und deren Anteil an der Gesamtverformung quantifiziert.

Abschließend enthält das Kapitel 7 eine Zusammenfassung der wesentlichen Ergebnisse dieser Arbeit und einen Ausblick hinsichtlich weiterführender Fragestellungen. 


\section{$2 \quad$ Stand der Forschung}

\subsection{Materialverhalten unter monoton steigender Beanspruchung}

Beton ist ein inhomogener Werkstoff dessen Materialverhalten auf unterschiedlichen Skalen problemorientiert betrachtet werden kann. Nach (Wittmann, 1986) ist eine Unterteilung in drei Skalen zweckmäßig: Mikro-, Meso- und Makroskala. Auf der Makroskala wird der Beton als homogenes Gefüge mit nichtlinearem Materialverhalten idealisiert. Sie wird vorrangig zur ingenieurmäßigen Beschreibung des Bauteil- bzw. Strukturverhaltens herangezogen. Die Mikroskala dient der Darstellung des Betons auf der Kristallebene, u. a. stehen die mechanischen Eigenschaften des Zementsteins und die Grenzflächen zwischen Zementstein und Gesteinskörnern im Fokus der Betrachtung. Das Risswachstum und die Verschiebungen zwischen Zementstein und Gesteinskörnern werden vorwiegend in der Mesoskala veranschaulicht. Die Risse sind häufig die Ursache für das nichtlineare Verhalten auf der Makroskala. Die Größenordnung dieses Bereichs liegt zwischen $10^{-4} \mathrm{~m}$ und $10^{-2} \mathrm{~m}$. Auf der Mesoskala wird der Beton als Zweiphasensystem bestehend aus Zementstein und Gesteinskörnern betrachtet, vgl. Abbildung 2.1. Dabei kann der Zementstein in die Bestandteile Zementgel, Feinanteile der Gesteinskörnung und Poren weiter unterteilt werden.

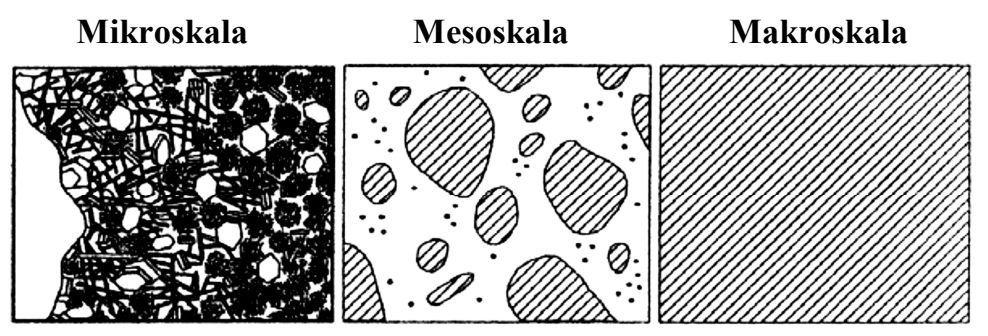

Abbildung 2.1: Betrachtungsskalen von Beton aus (Wittmann, 1986)

Zementstein und Gesteinskörner können sehr unterschiedliche Steifigkeiten und Festigkeiten besitzen, die das Materialverhalten des Betons wesentlich beeinflussen. Bei einem Normalbeton kann davon ausgegangen werden, dass die Gesteinskörner eine deutlich höhere Steifigkeit und Festigkeit aufweisen als der Zementstein. Eine äußerlich gleichmäßige Belastung führt damit zu einem ungleichmäßigen Kraftfluss in der Betonmatrix. So treten bereits bei äußerlich homogenen Druckbeanspruchungen, weit unterhalb der Materialfestigkeitsgrenze, im Zementstein Zugspannungen auf, die zur Rissbildung führen (Wischers \& Lusche, 1972a; Wischers \& Lusche, 1972b; Spooner \& Dougill, 1975). Die Anrisse treten insbesondere in den Kontaktzonen zwischen dem Zementstein und den Gesteinskörnern auf, vgl. Abbildung 2.2. (Rehm et al., 1977) konnte in diesem Zusammenhang darlegen, dass sich um die Gesteinskörner eine $10 \mu \mathrm{m}$ bis $20 \mu \mathrm{m}$ dicke Schicht ausbildet, dessen Zugfestigkeit niedriger ist als die des ungestörten Zementsteins. Darüber hinaus weist der unbelastete Beton bereits durch das Schwinden des Zementsteins sogenannte Schwindrisse in diesen Kontaktzonen auf (Smadi \& Slate, 1989; Siebel, 1989). Die Größe und die 
Verteilung der Schwindrisse sind von dem Schwindmaß des Zementsteins, der Haftzugfestigkeit zwischen Zementstein und Gesteinskörnern und dem Verhältnis der Elastizitätsmoduln von Zementstein und Gesteinskörnern abhängig. Bei steigender Druckbeanspruchung pflanzen sich die Risse in den Zementstein fort und laufen i. d. R. um die festeren Gesteinskörner herum. Im Gegensatz dazu sind hochfeste Betone durch eine geringere Steifigkeitsdifferenz und durch bessere Verbundeigenschaften zwischen Gesteinskörnern und Zementstein gekennzeichnet. Die Risse verlaufen dann durch Zementstein und Gesteinskorn hindurch.

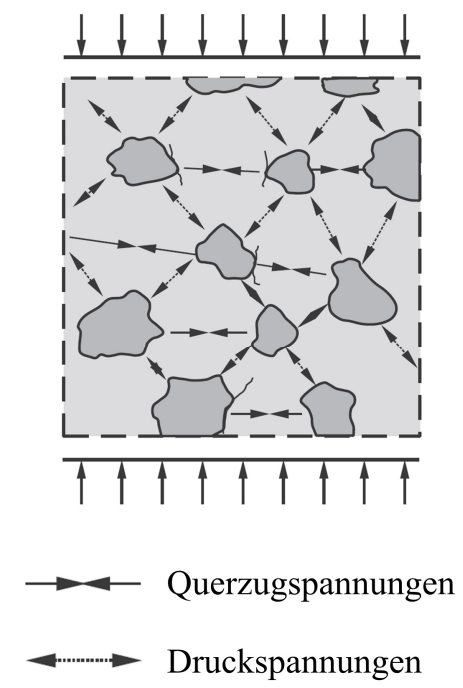

Abbildung 2.2: Modellvorstellung zur Rissbildung aus (Zilch \& Zehetmaier, 2010)

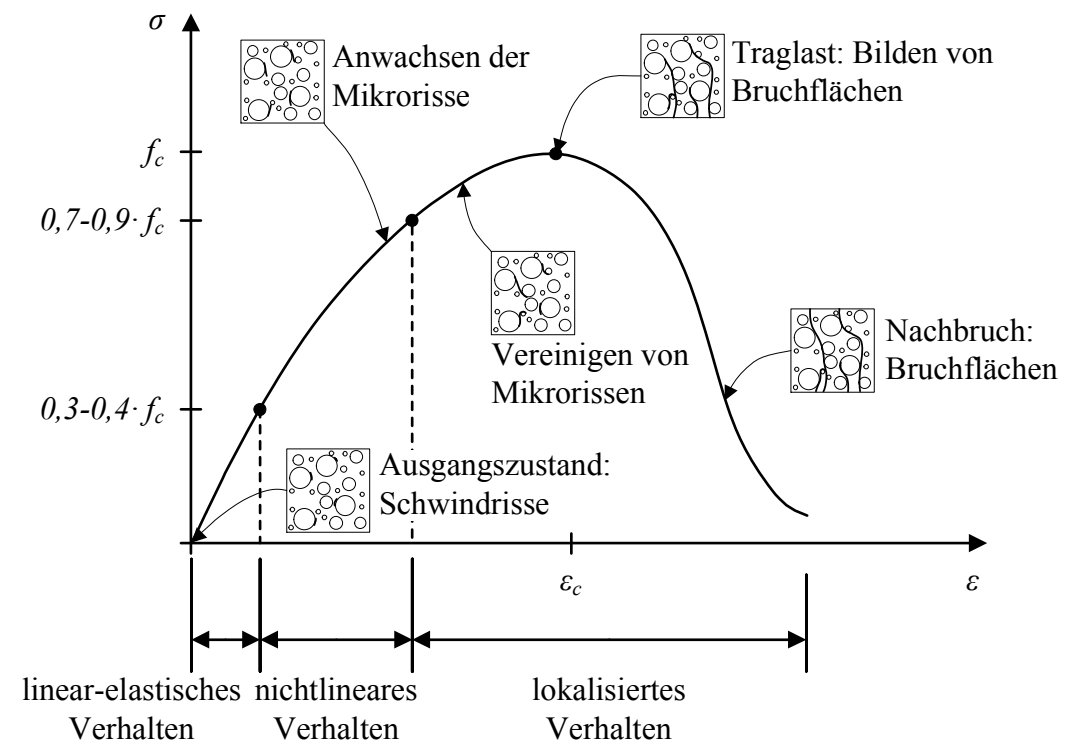

Abbildung 2.3: Spannungsdehnungslinie von druckbeanspruchtem Beton mit schematischer Darstellung der Rissbildung nach (Mehlhorn \& Kollegger, 1995)

Makroskopisch weist Normalbeton unter einer monoton steigenden Druckbeanspruchung bis etwa $30 \%$ bis $40 \%$ der Bruchspannung $f_{c}$ ein nahezu linear-elastisches Materialverhalten auf. Bei weiterer Laststeigerung wachsen die im Zementstein vorhandenen Schwindrisse an, und es bilden sich neue Mikrorisse. Mit der Beanspruchungshöhe nehmen die Anzahl und die Länge der Mikrorisse kontinuierlich zu (Smadi \& Slate, 1989). Die Risse führen zu schädigungsinduzierten plastischen Dehnungen, die im Spannungsdehnungsdiagramm als Steifigkeitsreduktion kenntlich werden. Ab etwa $70 \%$ bis $90 \%$ der Bruchspannung $f_{c}$, der sogenannten kritischen Spannung, vereinigen sich die Mikrorisse und es kommt zur Ausbildung von Bruchflächen (Newmann, 1965; van Mier et al., 1987). Bis zur kritischen Spannung ist das Verhältnis der Querdehnung zur Längsdehnung näherungsweise konstant, es liegt üblicherweise im Bereich von $v_{c}=0,14$ bis 0,26. Oberhalb der kritischen Spannung nimmt das Querdehnungsverhältnis überproportional zu und kann bis zum Erreichen der Bruchspannung ein Vielfaches seines Ursprungwertes annehmen (Ritter, 2014). Bei einer weggeregelten Versuchsdurchführung nehmen im anschließenden Bereich die Spannungen sukzessive ab, während die Dehnungen weiter zunehmen, vgl. Abbildung 2.3. In diesem Nachbruchbereich findet eine Lokalisierung der Schädigung statt. Das bedeutet, dass die Verformungen nunmehr von den Verschiebungen in den Bruchflächen dominiert werden, während in den übrigen Bereichen der Betonprobe eine Entlastung stattfindet (van Mier et al., 
1987; Markeset, 1993; Mehlhorn \& Kollegger, 1995; Pölling, 2000). Abweichend vom Eurocode 2 wird der Dehnungswert des Betons bei Erreichen der Bruchspannung $f_{c}$ im Rahmen dieser Arbeit als Bruchdehnung $\varepsilon_{c}$ bezeichnet.

Bei hochfesten Betonen ist der linear-elastische Bereich im Vergleich zu normalfesten Betonen, durch die geringere Steifigkeitsdifferenz zwischen Zementstein und Gesteinskörnern und durch die besseren Verbundeigenschaften, bis zu einem höheren Lastniveau gegeben. Gleichzeitig wird der abfallende Ast der Spannungsdehnungslinie mit zunehmender Betonfestigkeit immer steiler. Dies ist maßgeblich auf eine mit der Betonfestigkeit zunehmende Versprödung des Materials zurückzuführen.

Im Zuge der Lastaufbringung treten in Abhängigkeit der Belastungsgeschwindigkeit unterschiedlich große Dehnungen auf, vgl. Abbildung 2.4. Neben den elastischen und schädigungsinduzierten plastischen Dehnungen treten im Beton zeitabhängige Dehnungsanteile auf, die zu einem zeit- und spannungsabhängigen Verformungsverhalten führen. Das Materialverhalten wandelt sich somit von einer $\sigma$ - $\varepsilon$-Beziehung zu einer $\sigma$ - $\varepsilon$-t-Beziehung. Gleichzeitig sinkt die Festigkeit des Betons bei abnehmender Belastungsgeschwindigkeit. Sie nähert sich asymptotisch einem unteren Grenzwert, der Dauerstandfestigkeit, an. Diese liegt bei $80 \%$ bis $85 \%$ der Kurzzeitfestigkeit, vgl. Abbildung 2.5. Die Ursache wird in einem kontinuierlichen, zeitabhängigen Rissfortschritt ab dem Zeitpunkt des Überschreitens der Dauerstandfestigkeitsgrenze gesehen.

Die im Eurocode 2 verankerte Spannungsdehnungslinie des Betons gilt für den Fall, dass die Höchstlast innerhalb von 1 bis 2 Minuten erreicht wird (Reinhardt \& Hilsdorf, 2001; DAfStb, 2012). Das entspricht einer Belastungsgeschwindigkeit von ca. $0,5 \mathrm{~N} /\left(\mathrm{mm}^{2} \cdot \mathrm{s}\right.$ ) (DIN 1048-5, 1991). Dabei handelt es sich aus werkstoffspezifischer Sicht um eine willkürliche Festlegung, die primär aus versuchstechnischen Gründen getroffen wurde. Es wird vereinfachend angenommen, dass innerhalb dieser Zeitspanne vernachlässigbare zeitabhängige Verformungsanteile auftreten und das Materialverhalten somit näherungsweise durch eine $\sigma-\varepsilon$-Beziehung abgebildet werden kann.

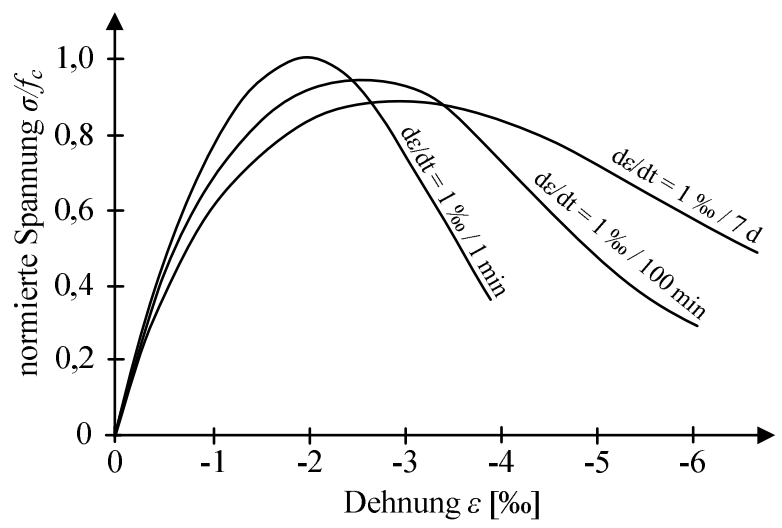

Abbildung 2.4: Spannungsdehnungslinie in Abhängigkeit von der Belastungsgeschwindigkeit nach (Rüsch, 1960)

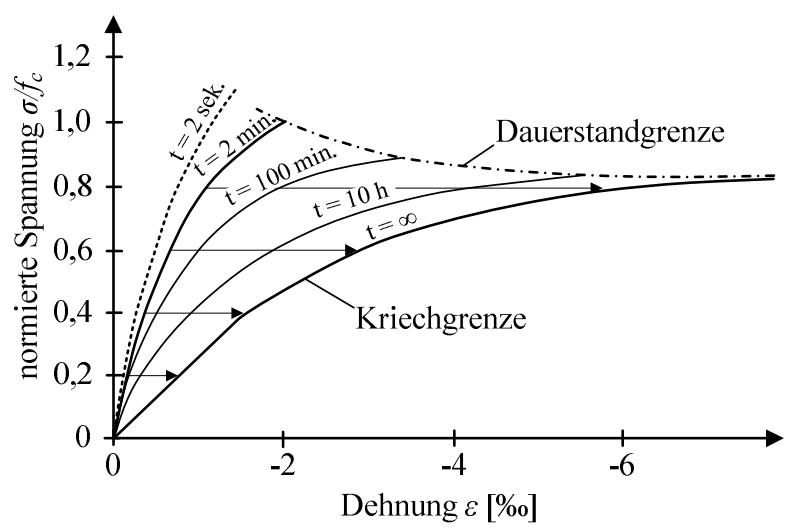

Abbildung 2.5: Dauerstandgrenze und Verformungen in Abhängigkeit von der Beanspruchungsgeschwindigkeit nach (Rasch, 1962) 


\subsection{Materialverhalten unter Dauerbeanspruchung}

\subsubsection{Phänomenologisches Materialverhalten}

Die Verformungskomponenten des Betons unter einer zeitlich begrenzten Druckbeanspruchung werden in Abbildung 2.6 dargestellt. Mit Aufbringen der Kriechspannung $\sigma_{c r}$ zum Zeitpunkt $t_{0}$ stellt sich zunächst eine sofortige Dehnung ein. Diese setzt sich entsprechend Abschnitt 2.1 bei einer ausreichend hohen Belastungsgeschwindigkeit vornehmlich aus elastischen $\varepsilon_{e l}\left(t_{0}\right)$ und schädigungsinduzierten plastischen Dehnungsanteilen $\varepsilon_{p l}\left(t_{0}\right)$ zusammen. Zeitlich verzögert entwickeln sich die Kriechdehnungen $\varepsilon_{c r}\left(t, t_{0}\right)$. Im Bereich der Kriechforschung wird jedoch allein zwischen elastischen Dehnungen und Kriechdehnungen differenziert. Der elastische Anteil bezeichnet entsprechend der Definition des Begriffes „elastisch“ nur jene Verformungen, die bei Entlastung einer Probe unmittelbar zurückgehen (Wriggers et al., 2005). Die mit der Belastung einhergehenden schädigungsinduzierten Dehnungen werden als Teil der Kriechdehnungen aufgefasst. In Abhängigkeit von der Belastungsdauer nehmen die Kriechdehnungen zu. Die Entlastung zum Zeitpunkt $t_{l}$ führt zu einer elastischen Rückverformung $\varepsilon_{e l}\left(t_{l}\right)$ des Betons. Infolge der Nacherhärtung des Betons ist diese geringer als die elastische Dehnung zu Belastungsbeginn. Zusätzlich geht ein Teil der allmählich auftretenden Kriechdehnungen, die zeitlich verzögerten Kriechdehnungen $\varepsilon_{v}$, zurück. Nach ausreichender Dauer verbleiben nur noch die irreversiblen Kriechdehnungen im Beton, die auch als Fließdehnungen $\varepsilon_{f}$ bezeichnet werden.
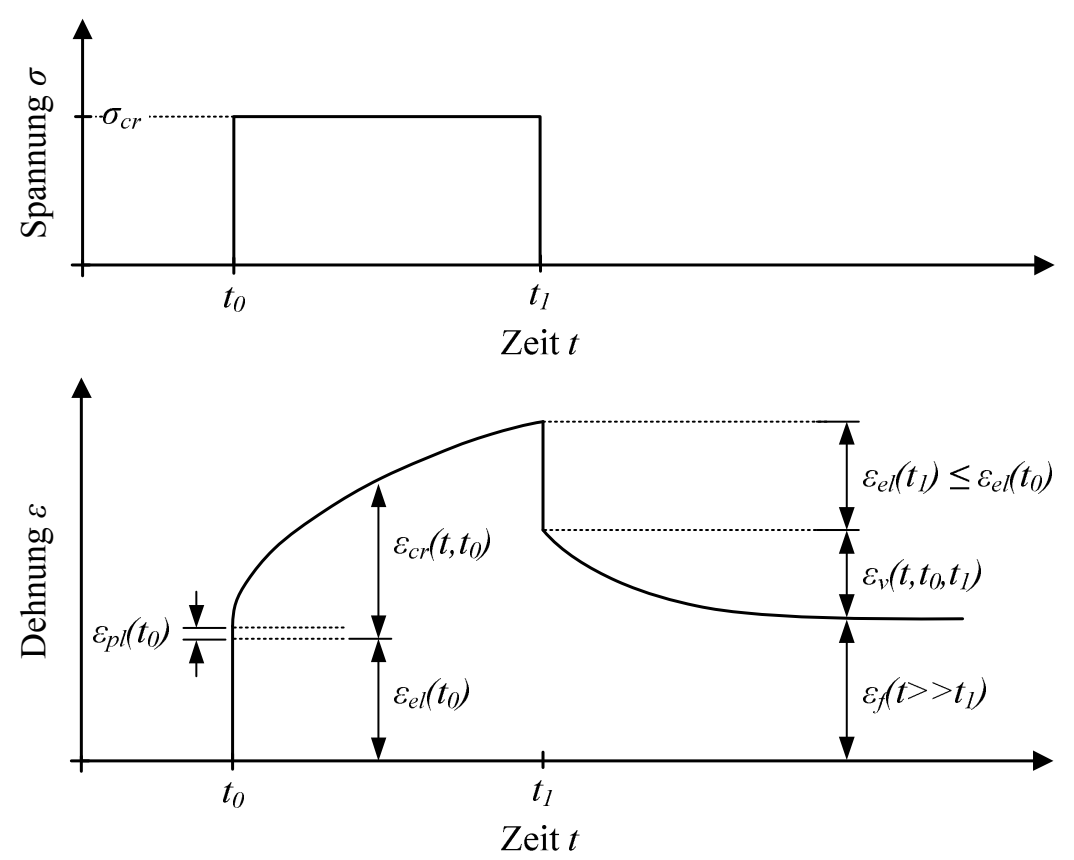

Abbildung 2.6: Dehnungskomponenten von Beton unter einer zeitlich begrenzten Last

Die Kriechdehnungen finden insbesondere im Zementstein statt und werden vom Wassergehalt des Zementsteins wesentlich beeinflusst. Die stattfindenden Prozesse können in die Anteile Grundkriechen und Trocknungskriechen unterteilt werden (Reinhardt \& Hilsdorf, 2001; Zilch \& Zehetmaier, 2010). Das Grundkriechen beschreibt den diffusionsunabhängigen Verformungs- 
anteil, der sich aus reversiblen und irreversiblen Verformungen zusammensetzt. Die reversiblen Verformungen werden auf die visko-elastischen Eigenschaften des Zementgels zurückgeführt und können bis zu $40 \%$ der sofortigen Dehnungen erreichen (Hilsdorf \& Müller, 1987). Der irreversible Verformungsanteil resultiert aus Partikelumlagerungen und Strukturveränderungen im Zementstein. Das Trocknungskriechen beinhaltet irreversible Verformungen, die mit der Vorstellung des Auspressens und Verdunstens von chemisch ungebundenem Wasser einer Probe während der Versuchsdurchführung einhergehen (Müller, 1986; Streit, 1991; Schwabach, 2005; Pfister, 2007).

Das Maß der auftretenden Kriechverformungen ist von einer Vielzahl an Einflussfaktoren abhängig, darunter fallen u. a. die Beanspruchungsdauer und -höhe, der Hydratationsgrad des Betons bei Belastungsbeginn, der Feuchtegehalt des Betons, die klimatischen Umgebungsbedingungen und die Betonzusammensetzung. Neben den lastabhängigen Kriechverformungen treten lastunabhängige Schwindverformungen während der Erhärtung und Austrocknung des Betons auf. Diese sind nicht Bestandteil der folgenden Ausführungen.

Unter hohen Dauerlasten beschreiben die Kriechdehnungen einen dreiphasigen Verformungsverlauf. Die Kriechphasen werden als Primär-, Sekundär- und Tertiärkriechen bezeichnet, vgl. Abbildung 2.7. Sie treten jedoch nur bei Dauerlasten oberhalb der Dauerstandfestigkeitsgrenze des Betons auf. Unter geringeren Spannungen tritt im Beton ausschließlich primäres Kriechen auf. Dieser Bereich ist durch eine abnehmende Kriechgeschwindigkeit gekennzeichnet. Bis heute ist allerdings nicht geklärt, ob die Kriechgeschwindigkeit für den Zeitpunkt $t \rightarrow \infty$ tatsächlich gegen null konvergiert und die Kriechverformungen damit einen Grenz- bzw. Endkriechwert anstreben, oder ob sich ein Sekundärkriechen einstellt und die Kriechgeschwindigkeit einen konstanten, wenn auch sehr kleinen Wert annimmt (Streit, 1991). In der Regel wird der Einfachheit halber ein Endkriechwert unterstellt.

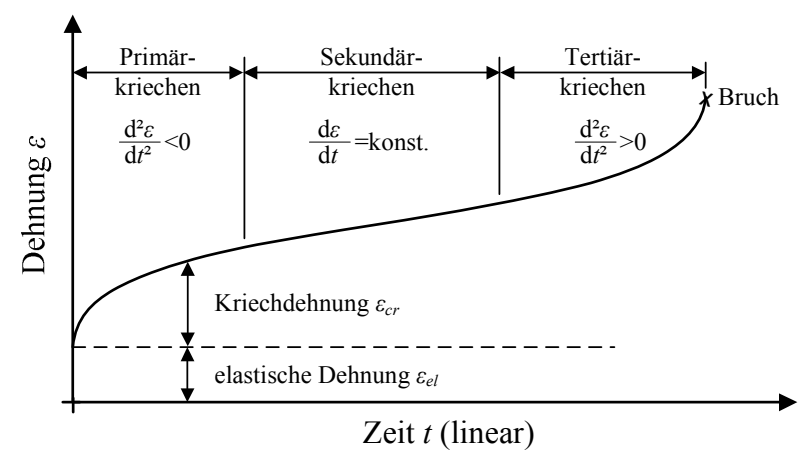

Abbildung 2.7: Kriechphasen bei Dauerlasten oberhalb der Dauerstandfestigkeit nach (Streit, 1991)

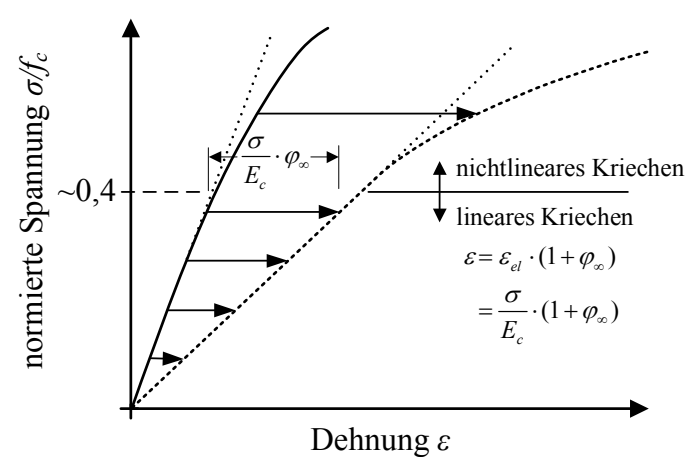

Abbildung 2.8: Lineares und nichtlineares Kriechen nach (Zilch \& Zehetmaier, 2010)

Bei Spannungen unterhalb von $40 \%$ bis $45 \%$ der Bruchspannung verhält sich der Endkriechwert näherungsweise proportional zur Beanspruchungshöhe bzw. elastischen Dehnung. Es wird vom linearen Kriechen gesprochen. Zurückgehend auf (Dischinger, 1937) wird der Endkriechwert über 
die dimensionslose Kriechzahl $\varphi_{\infty}$ beschrieben, diese quantifiziert das Verhältnis der Kriechdehnungen zum Zeitpunkt $t \rightarrow \infty$ zu den elastischen Dehnungen bei Belastungsbeginn.

$$
\varepsilon_{c r}(t \rightarrow \infty)=\varphi_{\infty} \cdot \varepsilon_{e l}\left(\sigma_{c r}\right)
$$

Der Kriechverlauf bzw. die Entwicklung der Kriechzahl $\varphi$ wird gewöhnlich als Funktion der Zeit $t$ und des Betonalters bei Belastungsbeginn $t_{0}$ aufgestellt. Die Verformungen des Betons unter Berücksichtigung der elastischen Dehnungen bei Belastungsbeginn ergeben sich damit zu:

$$
\varepsilon\left(t, t_{0}\right)=\varepsilon_{e l}\left(\sigma_{c r}\right) \cdot\left[1+\varphi\left(t, t_{0}\right)\right]=\frac{\sigma_{c r}}{E_{c}} \cdot\left[1+\varphi\left(t, t_{0}\right)\right]
$$

Mit Einführung der Komplianzfunktion der Form:

$$
J\left(t, t_{0}\right)=\frac{1}{E_{c}} \cdot\left[1+\varphi\left(t, t_{0}\right)\right]
$$

folgt aus Gleichung 2.2:

$$
\varepsilon\left(t, t_{0}\right)=\sigma_{c r} \cdot J\left(t, t_{0}\right)
$$

Die Komplianzfunktion beschreibt die gesamten spannungsinduzierten Verformungen infolge einer Einheitslast in Abhängigkeit von der Zeit und dem Betonalter bei Belastungsbeginn. Sie unterstreicht die angenommene Proportionalität zwischen Dehnung und Spannung im Bereich des linearen Kriechens. Bei Beanspruchungen oberhalb der Linearitätsgrenze weist der Beton durch vermehrte Mikrorissbildung und vermehrtes Mikrorisswachstum ein nichtlineares Kriechverhalten auf, d.h. die zeitabhängigen Verformungen nehmen mit der Beanspruchungshöhe überproportional zu, vgl. Abbildung 2.8. Diese überproportionale Verformungszunahme wird im Model Code 1990 und Model Code 2010 über eine Exponentialfunktion gemäß Gleichung 2.5 beschrieben:

$$
k_{\sigma}=e^{1,5 \cdot\left(\frac{\sigma_{c r}}{f_{c}}-0,4\right)} \text { für } 0,4 \cdot f_{c}<\sigma_{c r} \leq 0,6 \cdot f_{c}
$$

Die Kriechverformungen ergeben sich damit zu:

$$
\varepsilon\left(t, t_{0}\right)=\sigma_{c r} \cdot J\left(t, t_{0}\right) \cdot k_{\sigma}
$$

Überschreiten die Druckspannungen die Dauerstandfestigkeitsgrenze des Betons, geht das primäre Kriechen in sekundäres und tertiäres Kriechen über. Im letzteren beschleunigt sich der Prozess der Mikrorissbildung, und es kommt zur Ausbildung von Trennrissen, die schließlich zum Versagen des Probekörpers führen (Streit, 1991; Schwabach, 2005). In der tertiären Phase nimmt das Querdehnungsverhältnis, wie im monotonen Druckversuch nach Überschreiten der kritischen Spannung, bis zum Versagen des Probekörpers überproportional zu (Awad \& Hilsdorf, 1971).

Während eines Kriechversuchs werden die Betonproben bei einer Belastungsgeschwindigkeit von ca. $0,5 \mathrm{~N} /\left(\mathrm{mm}^{2} \cdot \mathrm{s}\right)$ bis zum Erreichen der Kriechlast belastet (DAfStb, 1991). Innerhalb dieser 
Belastungsphase treten elastische, schädigungsinduzierte und zum geringen Teil zeitabhängige Verformungen auf, vgl. Abbildung 2.5 und Abbildung 2.6. Anschließend ist die Verformungsentwicklung des Betonkörpers über die Versuchsdauer kontinuierlich aufzuzeichnen. Die Kriechdehnungen ergeben sich definitionsgemäß aus den Gesamtverformungen abzüglich der elastischen Anfangsverformung, die mithilfe des Elastizitätsmoduls des untersuchten Betons zu berechnen ist. Der Elastizitätsmodul ist nach (DIN 1048-5, 1991) bzw. (DIN EN 12390-13, 2014) zu bestimmen. Damit beinhalten die Kriechverformungen schädigungsinduzierte Anteile, die während der Belastungsphase auftreten, und viskose Verformungsanteile, die zeitabhängig auftreten. Der viskose Anteil setzt sich aus reversiblen und irreversiblen Anteilen zusammen und beinhaltet damit auch schädigungsinduzierte Anteile, die aus der andauernden Wirkung der Last herrühren.

\subsubsection{Empirische Kriechfunktionen}

In der Vergangenheit wurden empirische Ansatzfunktionen aufgestellt, um die zeitliche Entwicklung der Kriechverformungen $\varepsilon_{c r}$ bzw. der Kriechzahl $\varphi$ mathematisch zu beschreiben. Folgend wird eine kurze Auflistung der verbreitetsten Ansätze gegeben. Sie gelten für konstante Dauerlasten auf dem Gebrauchstauglichkeitsniveau. Es wird von einer kontinuierlich abnehmenden Kriechgeschwindigkeit entsprechend der primären Kriechphase ausgegangen. In den Ansätzen beschreibt $t_{0}$ das Betonalter bei Belastungsbeginn und der Ausdruck $\left(t-t_{0}\right)$ die Belastungsdauer. Die aufgeführten Konstanten $C, a, b, m$ und $n$ sind aus Versuchsergebnissen abzuleitende Parameter.

(Straub, 1931) und (Shank, 1935) wählten zur Beschreibung der Kriechverformungen einen Potenzansatz der Form:

$$
\varepsilon_{c r}\left(t-t_{0}\right)=C \cdot\left(t-t_{0}\right)^{m}
$$

Die Besonderheit des Potenzansatzes ist, dass dieser keinem Endwert entgegen strebt und somit auch keinem Endkriechwert unterliegt. (Bazant \& Osman, 1976) erweiterten diesen Ansatz in dem sogenannten Double Power Law, sodass der Kriechverlauf nicht nur von der Belastungsdauer, sondern auch von dem Betonalter bei Belastungsbeginn abhängig ist. Damit wird eine abnehmende Kriechfähigkeit mit zunehmendem Betonalter berücksichtigt.

$$
\varepsilon_{c r}\left(t-t_{0}\right)=C \cdot\left(t-t_{0}\right)^{m} \cdot t_{0}^{-n}
$$

(Dischinger, 1937), der auf Grundlage einer Literaturstudie seiner Zeit erkannte, dass sich die Kriechdehnungen proportional zur elastischen Dehnung verhalten, wählte zur Beschreibung des zeitlichen Verlaufs der Kriechzahl $\varphi$ eine Exponentialfunktion. Die allgemeine Form lautet:

$$
\varphi\left(t-t_{0}\right)=\varphi_{\infty} \cdot\left(1-e^{-m \cdot\left(t-t_{0}\right)^{n}}\right)
$$


Dischinger setzte die Konstanten $m$ und $n$ zu eins. Umfangreiche Versuchsdatenauswertungen in (Pfefferle, 1971) zeigen jedoch, dass insbesondere zu Versuchsbeginn größere Abweichungen zwischen experimentellen und rechnerischen Kriechwerten aufgetreten sind, die mit zunehmender Versuchsdauer abnehmen. Pfefferle zeigte anhand verschiedener Versuchsergebnisse, dass der Kriechverlauf mit $n=0,5$ zuverlässiger beschrieben werden kann. Die Abbildung 2.9 stellt die Verlaufsfunktion von Dischinger und dessen Anpassung von Pfefferle vergleichend gegenüber. $\mathrm{Zu}$ Belastungsbeginn resultieren eine höhere Kriechgeschwindigkeit und damit eine stärkere Verformungsentwicklung. Nach dieser Anfangsphase weist der Ansatz von Pfefferle eine geringere Kriechgeschwindigkeit auf, sodass sich die Verlaufsfunktion dem Endkriechwert langsamer asymptotisch annähert.

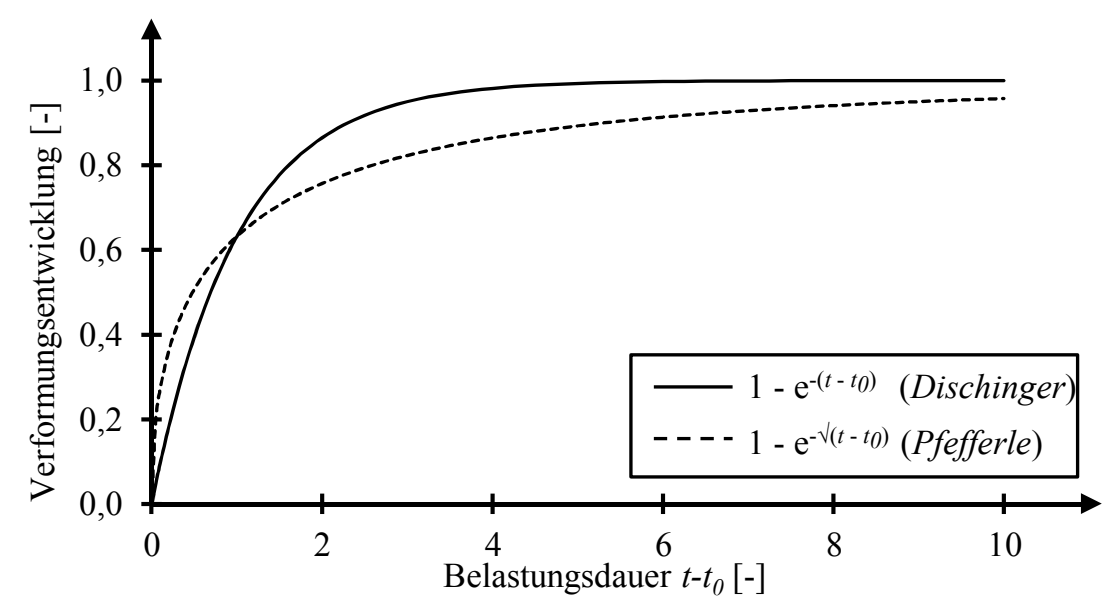

Abbildung 2.9: Verformungsansätze nach (Dischinger, 1937) und (Pfefferle, 1971)

(Ross, 1937) und (Lorman, 1940) schlugen eine Hyperbelfunktion zur mathematischen Beschreibung der Kriechverformungen vor. Die allgemeine Form lautet unter Verwendung der Kriechzahl:

$$
\varphi\left(t-t_{0}\right)=\varphi_{\infty} \cdot \frac{\left(t-t_{0}\right)}{a+b \cdot\left(t-t_{0}\right)}
$$

Die Hyperbelfunktion besitzt wie die Exponentialfunktion einen Endkriechwert der Größe $\varphi_{\infty}$. Im Model Code 1990 wird der Kriechverlauf über eine gegenüber Gleichung 2.10 angepasste Hyperbelfunktion der Form

$$
\varphi\left(t-t_{0}\right)=\varphi_{\infty} \cdot\left[\frac{\left(t-t_{0}\right)}{a+\left(t-t_{0}\right)}\right]^{m}
$$

beschrieben. Im Model Code 2010 wird der Kriechverlauf des Trocknungskriechens ebenfalls über eine Hyperbelfunktion erfasst. Der Kriechverlauf des Grundkriechens wird über eine Logarithmusfunktion abgebildet. Diese schlug (Hanson, 1953) erstmals vor, und sie besitzt wie die Potenzfunktion keinen Endwert:

$$
\varphi\left(t, t_{0}\right)=C \cdot \ln \left(\left(t-t_{0}\right)+1\right)
$$




\subsubsection{Rheologische Kriechansätze}

Neben den empirischen Ansatzfunktionen besteht das Bestreben, das Verformungsverhalten des Betons auf Grundlage rheologischer Modelle zu beschreiben. Die Rheologie beschäftigt sich mit dem Verformungs- und Fließverhalten materieller Systeme und umfasst Teilgebiete der Elastizitätstheorie, der Plastizitätstheorie und der Hydromechanik. Gegenüber den zuvor dargestellten empirischen Kriechfunktionen, versucht die Rheologie das Kriechverhalten des Betons durch die Kombination sogenannter Idealkörper abzubilden. Diese Idealkörper sind die Hookesche Feder, der Newtonsche Dämpfer und das St. Venantsche Element. Die Tabelle 2.1 stellt diese auch als Grundelemente bezeichneten Idealkörper und ihr Verformungsverhalten unter einer zeitlich begrenzten Last dar. Die Grundelemente können durch Parallel- oder Reihenschaltung zu Grundkörpern kombiniert werden. Diese vermögen theoretisch alle Arten des Verformungsverhaltens von Werkstoffen zu beschreiben. Über Gleichgewichts- und Verträglichkeitsbedingungen können mathematische Beziehungen aufgestellt werden, um damit das Spannungs-Dehnungs-Zeitverhalten zu analysieren.

Tabelle 2.1: Grundelemente der Rheologie (Boes, 1971)

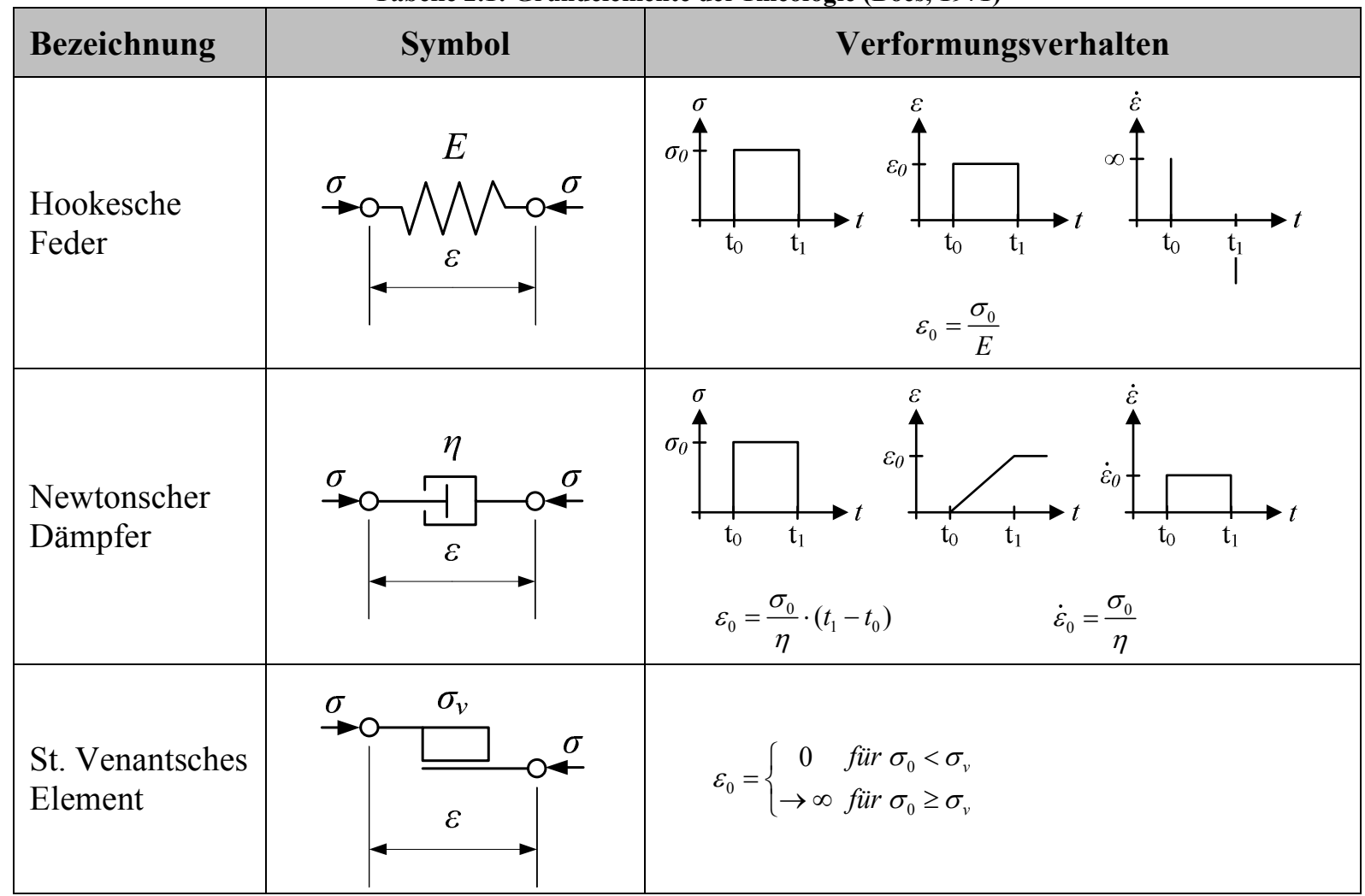

Die zur Modellierung des Betonkriechens bedeutendsten Grundkörper sind der Kelvin-(Voigt)Körper und der Maxwell-Körper, vgl. Tabelle 2.2. Von diesen vermag insbesondere der KelvinKörper, bestehend aus einer Hookeschen Feder und einem parallelgeschalteten Newtonschen Dämpfer, das lineare Kriechen des Betons abzubilden. Aus der Gleichgewichtsbedingung an dem System folgt eine Differentialgleichung 1. Ordnung, deren Lösung einer exponentiellen, zeitabhängigen Verformungsfunktion analog zu Gleichung 2.9 entspricht. Das Endkriechmaß ergibt sich spannungsabhängig aus dem Quotienten $\sigma_{0} / E$. Die Endkriechdehnung verhält sich 
damit proportional zur Kriechspannung. Das zeitlich verzögerte Verformungsverhalten wird durch die Dämpferkonstante $\eta$ bestimmt. Nach der Entlastung verformt sich der Kelvin-Körper wieder vollständig in seine Ausgangslage zurück. Der Maxwell-Körper besteht aus einer Hookeschen Feder, die das elastische Verformungsverhalten des Betons widerspiegelt, und einem in Reihe geschalteten Newtonschen Dämpfer, der die Kriechverformungen abbildet. Gegenüber dem Kelvin-Körper bleiben beim Maxwell-Körper nach der Entlastung die zeitabhängigen Verformungsanteile im System gespeichert. Das St. Venantsche Element, das im Grundsatz ein ideal plastisches Verhalten darstellt, spielt im Bereich des linearen Betonkriechens praktisch keine Rolle.

Tabelle 2.2: Grundkörper der Rheologie

\begin{tabular}{|c|c|c|}
\hline Bezeichnung & Symbol & Verformungsverhalten \\
\hline $\begin{array}{l}\text { Kelvin-(Voigt-) } \\
\text { Körper }\end{array}$ & 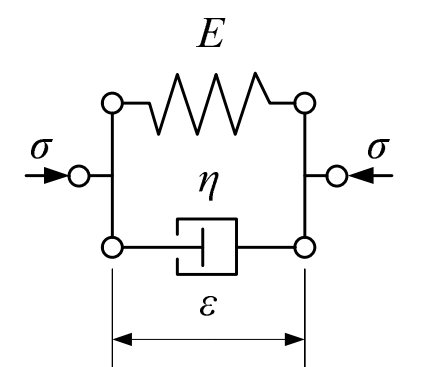 & 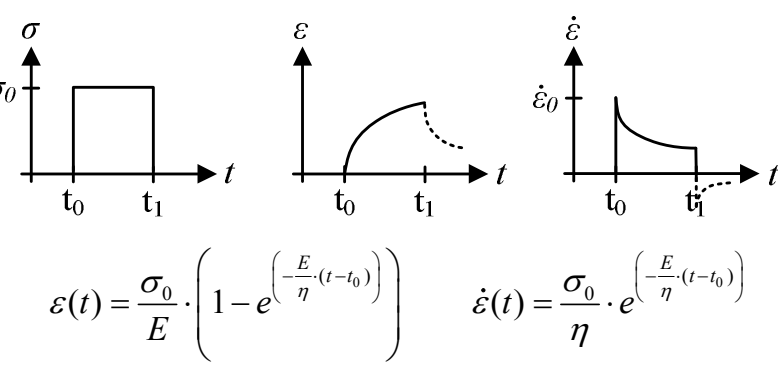 \\
\hline $\begin{array}{l}\text { Maxwell- } \\
\text { Körper }\end{array}$ & $\stackrel{\sigma}{\rightarrow} \bigwedge_{\varepsilon}^{E}$ & 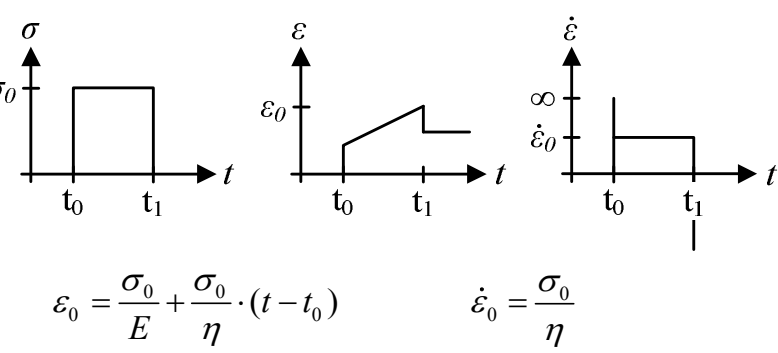 \\
\hline
\end{tabular}

Die Kopplung eines Kelvin-Körpers mit einer in Reihe geschalteten Hookeschen Feder stellt eines der einfachsten rheologischen Modelle zur Abbildung der Betondehnungen unter einer Dauerlast dar. Die Hookesche Feder bildet in dem Modell die unmittelbar auftretenden elastischen Verformungen des Betons bei Belastungsbeginn ab, während der Kelvin-Körper die zeitlich verzögert auftretenden Kriechverformungen abbildet. Bei diesem sogenannten PoyntingThomson-Modell (Abbildung 2.10 links) werden ausschließlich visko-elastische Kriecheigenschaften berücksichtigt. Nach der Entlastung verbleiben keine plastischen Verformungen im System gespeichert. Die Erweiterung des Poynting-Thomson-Modells um einen in Reihe geschalteten Newtonschen Dämpfer ermöglicht zusätzlich die Berücksichtigung von irreversiblen (Fließ-)Verformungen. Dieses Modell wird allgemein als Burgers-Modell (Abbildung 2.10 rechts) bezeichnet.

Durch Reihen- und Parallelschaltung mehrerer Grundelemente oder Grundkörper können verschiedene Prozesse des Kriechens separat behandelt und ihr Einfluss auf die Gesamtverformung individuell gewichtet werden (Boes, 1971; Müller, 1986; Bockhold \& 
Stangenberg, 2004). Die Gesamtverformungen ergeben sich aus der Summe der Verformungen der in Reihe geschalteten Grundelemente bzw. Grundkörper.
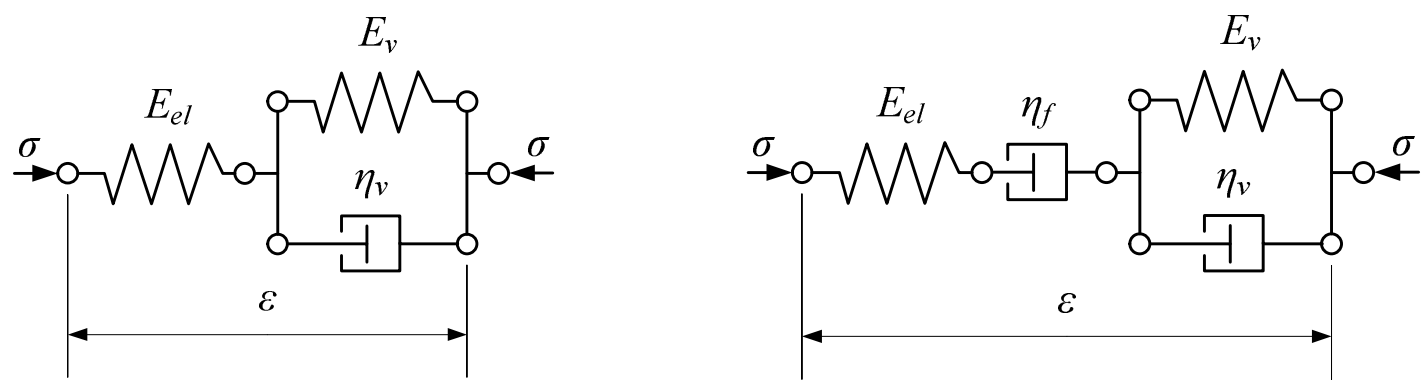

Abbildung 2.10: Poynting-Thomson-Modell (links) und Burgers-Modell (rechts)

Die Feder- und Dämpfereigenschaften der rheologischen Elemente können wie zuvor beschrieben konstant, aber auch in Abhängigkeit von der Spannung oder der Zeit formuliert werden. Die Leistungsfähigkeit der Modelle kann hierdurch wesentlich gesteigert werden. Wird die Hookesche Feder, die der Abbildung der sofortigen Dehnung dient, spannungsabhängig formuliert, so ermöglicht dies die Berücksichtigung des nichtlinearen Spannungs-Dehnungsverhaltens des Betons unter monoton steigender Beanspruchung entsprechend Abschnitt 2.1 (Shen, 1992). Die zeitabhängige Formulierung der Feder ermöglicht zudem die Berücksichtigung von Nacherhärtungseffekten. Gleiches gilt für die Federn und Dämpfer zur Abbildung der Kriechverformungen. Die Elemente können spannungs- und zeitabhängig formuliert werden, um einerseits die Nichtlinearität des Kriechens, aber auch die abnehmende Kriechfähigkeit des Betons mit zunehmendem Betonalter zu berücksichtigen.

\subsubsection{Kriechmodelle}

Die in Abschnitt 2.2.2 vorgestellten Kriechfunktionen vermögen bei geeigneter Wahl der Konstanten die Kriechverformungen eines Betons bzw. die Entwicklung der Kriechzahl unter einer konstanten Druckbeanspruchung, die zum Zeitpunkt $t_{0}$ aufgebracht wird, in guter Näherung widerzuspiegeln. Das Verhalten des Betons bzw. die Entwicklung der Kriechverformungen bei veränderlichen Spannungen oder abweichenden Belastungszeitpunkten vermögen diese Ansätze jedoch nicht $\mathrm{zu}$ beschreiben. Die Anwendung der Kriechfunktionen bei variierenden Belastungsbedingungen bzw. -altern wird durch die zugrunde gelegten Kriechmodelle bzw. Kriechtheorien definiert. Die folgend dargestellten Kriechmodelle stellen eine Auswahl der wichtigsten Kriechtheorien dar. Sie gelten unter der Voraussetzung des linearen Kriechens. Die Kriechtheorien können mit dem Verhalten der rheologischen Grundkörper und Modelle in Einklang gebracht werden.

\section{Theorie des Alterns (Dischinger-Ansatz)}

(Dischinger, 1937) ging davon aus, dass die Kriechfähigkeit des Betons allein vom Betonalter (zeitvariant) abhängt und durch eine ideale Kriechkurve (Whitney'sche Idealkurve) abgebildet werden kann. Als Idealkurve wird die Kriechkurve eines Betons aufgefasst, die sich unter einer 
frühestmöglichen Belastung ergibt. Der Kriechverlauf für eine zum Zeitpunkt $t_{0}$ aufgebrachte Belastung ergibt sich damit aus dem Verlauf der Idealkurve abzüglich der Verformungen, die bis zum Belastungsbeginn $t_{0}$ bereits eingetreten wären.

$$
\varphi\left(t, t_{0}\right)=\varphi(t)-\varphi\left(t_{0}\right)
$$

Die Kriechtheorie kann entsprechend Abbildung 2.11 (links) durch vertikales Verschieben der Idealkurve visualisiert werden. Das wesentliche Merkmal ist, dass der Einfluss des Betonalters und der zeitliche Verlauf der Kriechverformungen über die gleiche Funktion beschrieben werden.

Der Ansatz ermöglicht eine relativ einfache Verformungsvorhersage bei veränderlichen Spannungen. Jedoch beinhaltet er eine zu ausgeprägte Alterung des Betons, sodass vor allem bei hohen Betonaltern bei Belastungsbeginn deutlich zu kleine Kriechverformungen vorhergesagt werden. Des Weiteren hat der Ansatz zur Folge, dass bei einer Entlastung keine Kriecherholung beschrieben wird. Der Kriechvorgang wird damit als irreversibler Verformungsvorgang interpretiert. Wird abweichend von Abbildung 2.11 (links) der Betonkörper zum Zeitpunkt $t_{2}$ nicht weiter belastet, sondern vollständig entlastet, so ergeben sich die verbleibenden Kriechverformungen zu:

$$
\begin{aligned}
\varepsilon_{c r}(t) & =\varepsilon_{e l}\left(\sigma_{c r}\right) \cdot\left[1+\varphi\left(t, t_{1}\right)\right]-\varepsilon_{e l}\left(\sigma_{c r}\right) \cdot\left[1+\varphi\left(t, t_{2}\right)\right] \\
& =\varepsilon_{e l}\left(\sigma_{c r}\right) \cdot\left[1+\varphi(t)-\varphi\left(t_{1}\right)\right]-\varepsilon_{e l}\left(\sigma_{c r}\right) \cdot\left[1+\varphi(t)-\varphi\left(t_{2}\right)\right] \\
& =\varepsilon_{e l}\left(\sigma_{c r}\right) \cdot\left[\varphi\left(t_{2}\right)-\varphi\left(t_{1}\right)\right]
\end{aligned}
$$

Damit verbleiben die während der Belastungsdauer $\left(t_{2}-t_{1}\right)$ aufgetretenen Kriechverformungen im Betonkörper eingeprägt. Ausschließlich der elastische Dehnungsanteil verformt sich bei dieser Betrachtungsweise zurück.

Die Theorie des Alterns kann hinsichtlich der rheologischen Betrachtungsweise mithilfe eines Maxwell-Körpers abgebildet werden. Die Steifigkeit der Feder ist als Konstante aufzufassen. Der Dämpfer ist in Abhängigkeit der Zeit versteifend $\mathrm{zu}$ definieren, um die abnehmende Kriechfähigkeit des Betons mit zunehmenden Betonalter abzubilden. Die Differenzialgleichung des Maxwell-Körpers lautet damit:

$$
\frac{d \varepsilon}{d t}=\frac{1}{E} \cdot \frac{d \sigma}{d t}+\frac{\sigma}{\eta(t)}
$$

(Dischinger, 1937) formulierte die Differentialgleichung der Gesamtverformungen entsprechend der Theorie des Alterns zu:

$$
\frac{d \varepsilon}{d t}=\frac{1}{E} \cdot \frac{d \sigma}{d t}+\frac{\sigma}{E} \cdot \frac{d \varphi(t)}{d t}
$$

Durch Gleichsetzen der Gleichungen 2.15 und 2.16 folgt der Zusammenhang zwischen der Kriechzahl und den Feder- und Dämpfereigenschaften des rheologischen Maxwell-Modells.

$$
\varphi(t)=E \cdot \int \frac{1}{\eta(t)} d t
$$



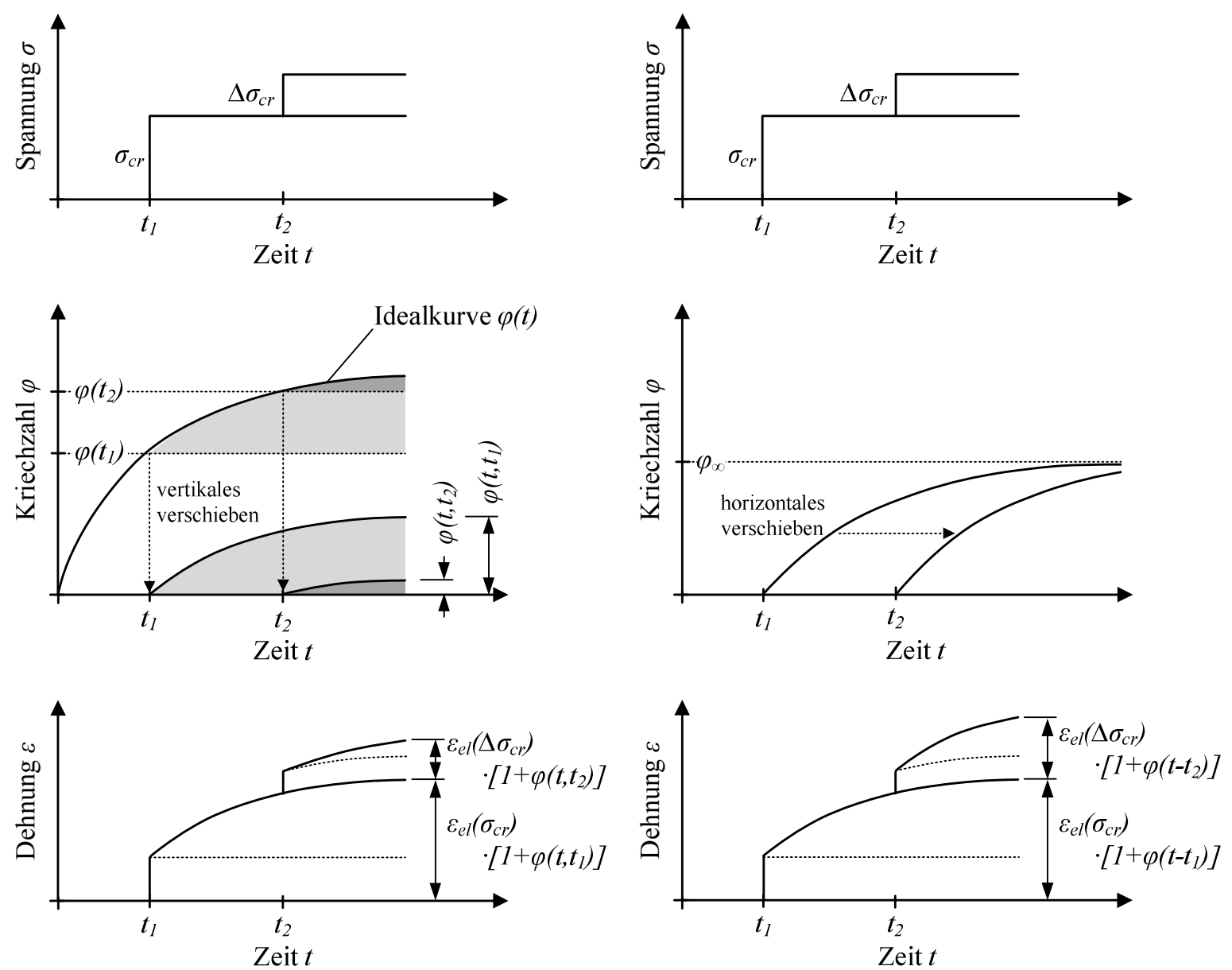

Abbildung 2.11: Theorie des Alterns (links) und Theorie der linearen Viskoelastizität (rechts) nach (Zilch \& Zehetmaier, 2010)

\section{Theorie der linearen Viskoelastizität}

Dieser Ansatz unterstellt dem Beton lineare visko-elastische Eigenschaften. Es findet gegenüber dem Dischinger-Ansatz keine Alterung des Materials statt, sondern es wird von zeitlich unabhängigen (zeitinvarianten) Kriecheigenschaften ausgegangen. Der Kriechbeiwert kann damit allein in Abhängigkeit der Belastungsdauer ausgedrückt werden. Damit nähern sich die Kriechverläufe unabhängig vom Betonalter immer asymptotisch dem gleichen Endkriechwert $\varphi_{\infty}$ an.

$$
\varphi\left(t, t_{0}\right)=\varphi\left(t-t_{0}\right)
$$

Die Theorie der linearen Viskoelastizität kann entsprechend Abbildung 2.11 (rechts) durch horizontales Verschieben der Kriechkurve auf der Zeitachse visualisiert werden. Der Ansatz liefert für Betone mit hohen Belastungsaltern zu große Kriechverformungen. Gleichzeitig werden keine irreversiblen Kriechverformungen berücksichtigt. Wird der Betonkörper abweichend zu Abbildung 2.11 (rechts) zum Zeitpunkt $t_{2}$ vollständig entlastet, ergeben sich die Kriechverformungen zu: 


$$
\begin{aligned}
\varepsilon_{c r}(t) & =\varepsilon_{e l}\left(\sigma_{c r}\right) \cdot\left[1+\varphi\left(t, t_{1}\right)\right]-\varepsilon_{e l}\left(\sigma_{c r}\right) \cdot\left[1+\varphi\left(t, t_{2}\right)\right] \\
& =\varepsilon_{e l}\left(\sigma_{c r}\right) \cdot\left[\varphi\left(t-t_{1}\right)-\varphi\left(t-t_{2}\right)\right]
\end{aligned}
$$

Da die Kriechfunktion zum Zeitpunkt $t \rightarrow \infty$ den Endkriechwert $\varphi_{\infty}$ annimmt, folgt:

$$
\varepsilon_{c r}(t \rightarrow \infty)=\varepsilon_{e l}\left(\sigma_{c r}\right) \cdot\left[\varphi_{\infty}-\varphi_{\infty}\right]=0
$$

Der Ansatz der linearen Viskoelastizität kann durch einen Kelvin-Körper mit konstanten Federund Dämpfereigenschaften abgebildet werden. Durch die Parallelschaltung von Feder und Dämpfer sind die Dehnungen in den beiden Elementen stets gleich. Gleichzeitig muss die Summe der inneren Spannungen immer der einwirkenden Spannung entsprechen. Aus dem Kräftegleichgewicht folgt eine Differentialgleichung 1. Ordnung der Form:

$$
\sigma=E \cdot \varepsilon+\eta \cdot \frac{d \varepsilon}{d t}
$$

Die Differentialgleichung kann für einen Belastungsbeginn zum Zeitpunkt $t_{0}$ analytisch gelöst werden. Die Kriechverformungen folgen entsprechend Gleichung 2.22 einem exponentiellen Verlauf.

$$
\varepsilon_{c r}=\frac{\sigma}{E} \cdot\left(1-e^{-\frac{E}{\eta} \cdot\left(t-t_{0}\right)}\right)
$$

Die Funktion nähert sich für $\mathrm{t} \rightarrow \infty$ asymptotisch dem Endkriechwert $\sigma / E$ an. Die Kriechgeschwindigkeit wird durch den Quotienten $E / \eta$ und durch die Belastungsdauer $\left(t-t_{0}\right)$ bestimmt. Eine Spannungsänderung des Systems bewirkt den Neubeginn des Kriechens und somit auch das vollständige Rückkriechen bei Entlastung.

\section{Produkt- und Summationsansätze}

Die zuvor beschriebenen Modelle ermöglichen die einfache mathematische Beschreibung des Kriechens bei variierenden Betonaltern oder Beanspruchungszuständen. Die Realität liegt jedoch zwischen diesen beiden Ansätzen. So wird bei dem Modell des affinen Kriechens die Kriechfunktion als Produkt aus einer zeitvarianten Alterungsfunktion (Theorie des Alterns) und einer zeitinvarianten Zeitdauerfunktion (Theorie der linearen Viskoelastizität) beschrieben. Damit wird das Kriechen des Betons von zwei unabhängigen Zeitfunktionen separat berücksichtigt. Infolge der multiplikativen Verknüpfung wird dieser Ansatz auch als Produktansatz bezeichnet. Diese Vorgehensweise ist u. a. im Model Code 1990 verankert. Die Endkriechzahl unterliegt dort einem alterungsabhängigen Ansatz, während die Verlaufsfunktion in Abhängigkeit der Belastungsdauer formuliert ist, vgl. Gleichung 2.23.

$$
\varphi\left(t, t_{0}\right)=\varphi_{\infty}\left(t_{0}\right) \cdot \beta\left(t-t_{0}\right)
$$

Nach der Entlastung vermag dieser Ansatz durch die Alterungsfunktion reversible als auch irreversible Verformungen vorherzusagen. Hinsichtlich der rheologischen Betrachtungsweise 
entspricht dieser Kriechansatz dem Kelvin-Modell, jedoch mit alterungsabhängigen Feder- und Dämpfereigenschaften.

Bei dem Summationsansatz wird der Dischinger-Ansatz, der das Kriechen zeitvariant betrachtet, um ein zeitinvariantes Glied erweitert. Dementsprechend wird der Ansatz auch Erweiterter Dischinger-Ansatz genannt. Mit diesem bestimmt sich die Kriechzahl $\varphi$ aus der Summe der verzögert-elastischen Verformungen $\varphi_{v}$ und der irreversiblen Fließverformungen $\varphi_{f}$.

$$
\varphi\left(t, t_{0}\right)=\varphi_{f}(t)-\varphi_{f}\left(t_{0}\right)+\varphi_{v}\left(t-t_{0}\right)
$$

Damit kann das Kriechen gegenüber dem Dischinger-Ansatz nicht nur als rein irreversibler Prozess aufgefasst werden, sondern das im Experiment beobachtete reversible und irreversible Verformungsverhalten kann nachempfunden werden. Rheologisch betrachtet entspricht dieser Ansatz dem Burgers-Modell mit alterungsabhängigen Feder- und Dämpfereigenschaften.

Veröffentlichungen jüngeren Datums konzentrieren sich insbesondere auf die Verbesserung und Verfeinerung dieser Ansätze. So werden beispielsweise beim Summationsansatz die Fließverformungen in Grund- und Trocknungskriechen aufgespalten oder der Produktansatz um weitere Glieder erweitert (Boes, 1971; Müller, 1986; CEB, 1990; Anders, 2012). So wird im Model Code 2010 die Kriechfunktion als Summenfunktion aus Grund- und Trocknungskriechen bestimmt, die jeweils einem Produktansatz unterliegen.

Als Erweiterung ist ebenso die Verfestigungstheorie (Solidification Theory) von (Bazant \& Prasannan, 1989) zu sehen. Bei dieser wird die Alterung des Betons als Volumenwachstum von lastabtragenden Zementhydraten betrachtet, das selbst nicht altert und als viskoelastisches Material behandelt wird. Der Ansatz versucht, Widersprüche hinsichtlich der thermodynamischen Betrachtungsweise zu lösen. So werden beim Kriechen die Materialparameter in Abhängigkeit von der Zeit definiert. Die Gesetze der Thermodynamik können jedoch nur für Systeme mit zeitunabhängigen Eigenschaften formuliert werden. Grundsätzlich fußt das Modell auf einem Summationsansatz unter Berücksichtigung visko-elastischer Verformungen und irreversibler Fließverformungen, die durch ein rheologisches Modell bestehend aus n-Kelvin-Körpern abgebildet werden. Da der Hydratationsprozess des Betons nach ca. einem Jahr weitestgehend abgeschlossen ist, wurde zur Verbesserung der Vorhersagegenauigkeit der langzeitigen Kriechverformungen in (Bazant et al., 1997) die Verfestigungstheorie zur sogenannten Mikrovorspannungs-Verfestigungstheorie (Microprestressing-Solidification Theory) erweitert.

\section{Superposition der Kriechdehnungen}

Bei veränderlichen Spannungsverläufen sind zur ingenieurmäßigen Verformungsvorhersage die aus den einzelnen Spannungsinkrementen zu erwartenden Kriechdehnungen zu superponieren. Im Allgemeinen wird auf das Superpositionsprinzip von Boltzmann zurückgegriffen. Dieses übertrugen von Maslov und McHenry erstmals auf das Betonkriechen (Shen, 1992; Anders, 2012). Das Superpositionsprinzip lässt sich auf zwei verschiedene Arten anwenden, die in Abbildung 
2.12 dargestellt sind. Zur Veranschaulichung der horizontalen Superpositionsmethode wird davon ausgegangen, dass der Beton zum Zeitpunkt $t_{0}$ mit der Spannung $\sigma\left(t_{0}\right)$ beansprucht wird. Der folgende Spannungsverlauf wird horizontal in $n$ Sprungfunktionen der Größe $\Delta \sigma\left(t_{i}\right)$ unterteilt. Die aus den einzelnen Spannungsinkrementen resultierenden Gesamtverformungen können über die folgende Näherung berechnet werden:

$$
\varepsilon(t)=\sigma\left(t_{0}\right) \cdot J\left(t, t_{0}\right)+\sum_{i=1}^{n} \Delta \sigma\left(t_{i}\right) \cdot J\left(t, t_{i}\right)
$$

Mit der Kriechfunktion anstelle der Komplianzfunktion lassen sich die Kriechverformungen unter veränderlichen Spannungen wie folgt berechnen:

$$
\varepsilon_{c r}(t)=\sigma\left(t_{0}\right) \cdot \varphi\left(t, t_{0}\right)+\sum_{i=1}^{n} \Delta \sigma\left(t_{i}\right) \cdot \varphi\left(t, t_{i}\right)
$$

Bei der vertikalen Superpositionsmethode wird der Spannungsverlauf in $n$,impulsförmige“ Intervalle der mittleren Größe $\sigma\left(t_{i}^{\prime}\right)$ und der Zeitdauer $\Delta \tau_{i}$ unterteilt. Der Verformungsverlauf errechnet sich aus der Summe der in den einzelnen Zeitschritten auftretenden inkrementellen Dehnungsänderungen unter der gemittelten Spannung im Zeitschritt:

$$
\varepsilon(t)=\sum_{i=0}^{n} \sigma\left(t_{i}^{\prime}\right) \cdot\left[J\left(t, t_{i}\right)-J\left(t, t_{i+1}\right)\right]
$$

bzw.

$$
\varepsilon_{c r}(t)=\sum_{i=0}^{n} \sigma\left(t_{i}^{\prime}\right) \cdot\left[\varphi\left(t, t_{i}\right)-\varphi\left(t, t_{i+1}\right)\right]
$$

Bei einer linearen Beziehung zwischen Kriechdehnung und Kriechspannung können beide Methoden gleichwertig angewendet werden. Wenn zwischen der Kriechdehnung und der Kriechspannung eine nichtlineare Beziehung besteht oder weitere Einflussfaktoren auf das Kriechverhalten während der Beanspruchungsdauer einwirken (Temperatur, Luftfeuchte), so muss die vertikale Superpositionsmethode angewendet werden.
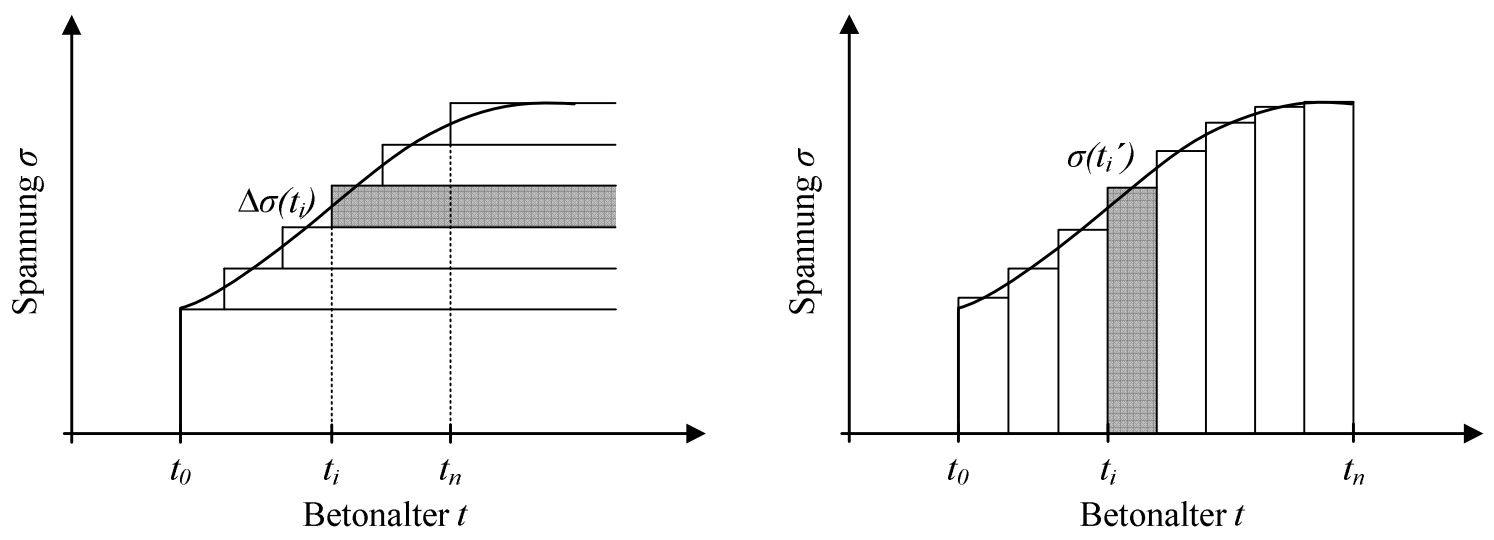

Abbildung 2.12: Horizontale (links) und vertikale Superpositionsmethode (rechts) bei einem veränderlichen Spannungsverlauf nach (Anders, 2012) 


\subsection{Materialverhalten unter zyklischer, verformungsgeregelter Beanspruchung}

Untersuchungen zum Tragverhalten von Beton unter zyklischer, verformungsgeregelter Beanspruchung wurden anfänglich im Zusammenhang mit Forschungsaktivitäten zur Erdbebensicherheit durchgeführt. In den Versuchsszenarien wurde der gesamte Dehnungsbereich des druckbeanspruchten Betons in wenigen Be- und Entlastungszyklen verformungsgeregelt abgefahren. Als besonderes Merkmal ergaben sich Hystereseschleifen, die alle von einer umhüllenden Kurve eingeschlossen wurden, die der Spannungsdehnungslinie unter monoton steigender Beanspruchung glich (Karsan \& Jirsa, 1969; Spooner et al., 1976; Bahn \& Hsu, 1998). In der Abbildung 2.13 wird dieser typische Hystereseverlauf dargestellt. Es zeigt sich, dass mit zunehmender Lastzyklenzahl die Festigkeit entsprechend der einhüllenden Spannungsdehnungslinie abnimmt und die Verformungen im Material ansteigen. Die abnehmende Neigung der Be- und Entlastungspfade deuten auf eine zunehmende Steifigkeitsdegradation infolge fortschreitender Materialschädigung hin. Die zunehmenden plastischen Dehnungen bei vollständiger Entlastung sind die Folge der Materialschädigung sowie viskoser Verformungen. Mit der mathematischen Beschreibung der Be- und Entlastungskurven befassten sich u. a. (Sinha et al., 1964), (Yankelevsky \& Reinhardt, 1987) und (Bahn \& Hsu, 1998).

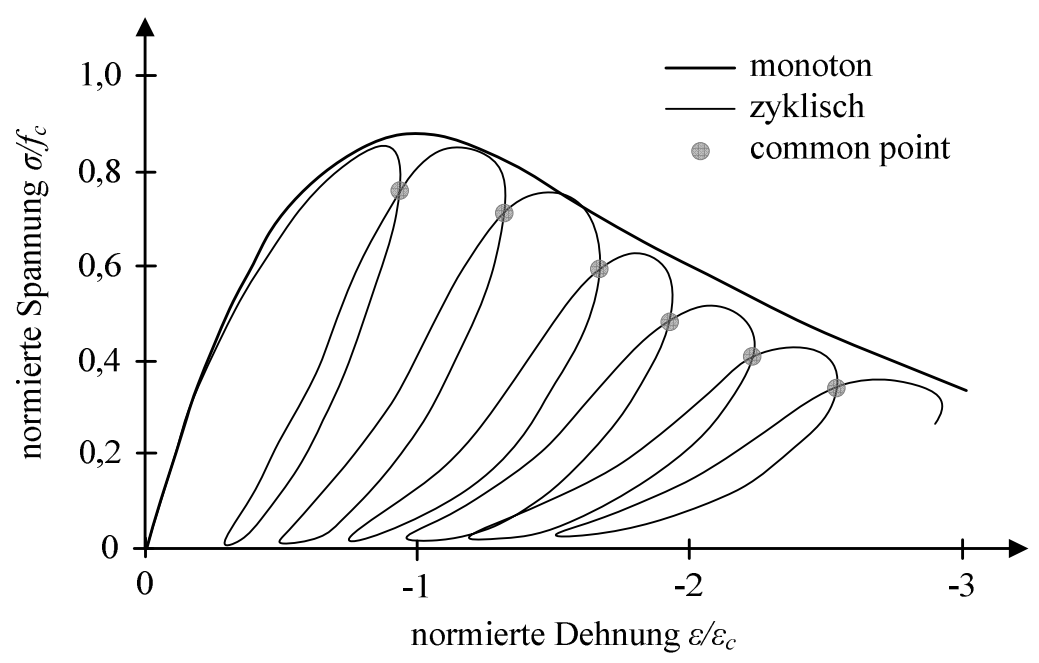

Abbildung 2.13: Spannungsdehnungslinie von Beton bei niederzyklischer Druckbeanspruchung nach (Karsan \& Jirsa, 1969)

Die Steifigkeitsabnahme kann, wie unter monoton steigender Beanspruchung, auf das Wachstum und die Vereinigung von Mikrorissen zurückgeführt werden. Schallemissionsanalysen von (Spooner \& Dougill, 1975) bestätigen diese Annahme. Die Entstehung von Rissen wird von hochfrequenten Schallimpulsen begleitet, die sich im Werkstoff als Wellen ausbreiten und mittels geeigneter Messwertaufnehmer an der Probenoberfläche detektieren lassen (Brecht et al., 1973; Krümmeling, 1999; Finck, 2005). (Spooner \& Dougill, 1975) stellten in ihren Untersuchungen fest, dass merkliche Schallemissionen nur während des ersten Belastungsastes und wenn nach einer Entlastung die Dehnungen des folgenden Belastungsastes den „Common Point“ überschritten emittiert wurden. Der „Common Point“ ist als Schnittpunkt zwischen dem 
Belastungsast und dem vorhergehenden Entlastungsast definiert, vgl. Abbildung 2.13. Im Zuge der Entlastung und der Wiederbelastung bis zum „Common Point“ wurden keine merklichen Schallemissionen detektiert. Hieraus wurde gefolgert, dass nur eine Wiederbelastung über das vorherige Dehnungsmaß hinaus eine Steifigkeitsabnahme bzw. Materialschädigung hervorruft.

Untersuchungen von (Karsan \& Jirsa, 1969) können diese These nicht grundsätzlich bestätigen. In zyklischen, verformungsgeregelten Versuchen wurden Probekörper nach einer gewissen Lastwechselzahl nicht mehr bis zum eintretenden Spannungsabfall belastet, sondern wiederholt bis zum „Common Point“. Es zeigte sich, dass die Spannungen und Dehnungen der sich mit jedem Lastwechsel neu einstellenden „Common Points“ abnahmen und einem „Stability Point“ anstrebten, vgl. Abbildung 2.14. Es ist somit anzunehmen, dass auch die Wiederbelastung bis zum vorherigen Dehnungswert eine geringe Materialschädigung hervorruft.

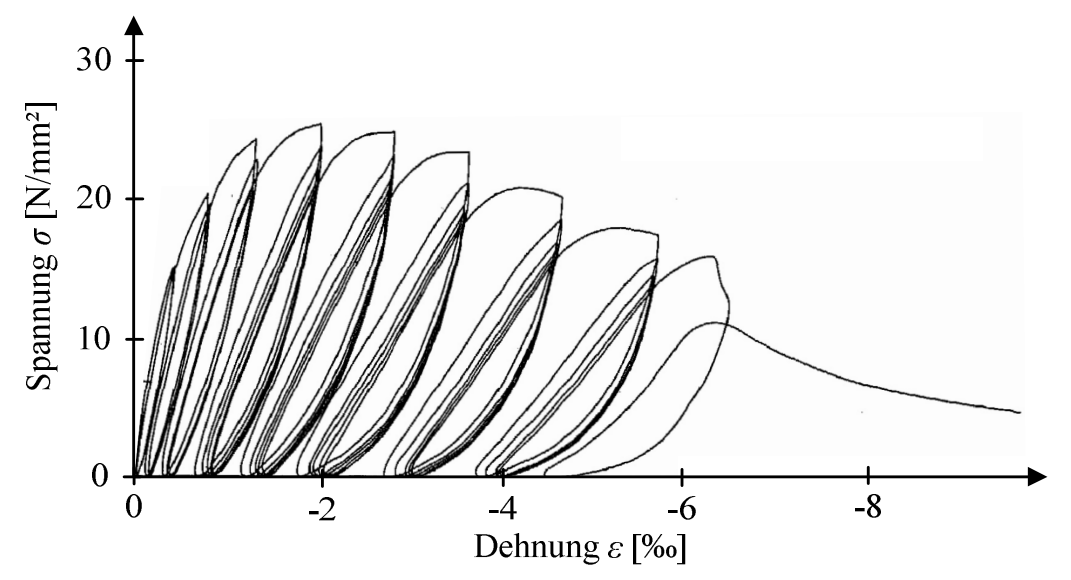

Abbildung 2.14: Entwicklung des „Stability Points“ nach (Maher \& Darwin, 1982)

Auf Grundlage des dargelegten Materialverhaltens wurden die sogenannten Envelope-Konzepte entwickelt. Diese betrachten die Spannungsdehnungslinie unter monoton steigender Beanspruchung als Grenzlinie für das Dehnungsverhalten des Betons unabhängig von der Belastungsart und Belastungsreihenfolge. Es ist jedoch $\mathrm{zu}$ beachten, dass der Verlauf der Spannungsdehnungslinie von der Belastungsgeschwindigkeit signifikant abhängig ist, vgl. Abbildung 2.4. Die Gegenüberstellung von Spannungsdehnungslinien unter monoton steigender Beanspruchung und den Verformungsverläufen bzw. Versagensdehnungen beliebiger Beanspruchungsszenarien setzt somit annähernd gleiche Beanspruchungsdauern und Beanspruchungsniveaus voraus. Dieser Grundsatz ist in den zuvor zitierten Untersuchungen überwiegend eingehalten. Der gesamte Dehnungsverlauf wurde innerhalb nur weniger Lastzyklen abgefahren. Die zyklischen Versuche dauerten damit nur unwesentlich länger als die unter monoton steigender Beanspruchung. Dementsprechend weisen (Sinha et al., 1964) und (Maher \& Darwin, 1982) darauf hin, dass der abfallende Ast der Envelope-Kurve flacher und höher verlaufen kann als die Spannungsdehnungslinie unter monoton steigender Belastung, wenn die Versuchsdauer im zyklischen Versuch wesentlich größer ist als unter monoton steigender Beanspruchung. Sie begründen dies mit größeren viskosen Dehnungen infolge der höheren Versuchsdauer im zyklischen Versuch. 


\subsection{Materialverhalten unter zyklischer, kraftgeregelter Beanspruchung}

\subsubsection{Allgemeines und Grundlagen}

Der Ermüdungswiderstand von Beton wird in kraftgeregelten Ermüdungsversuchen bestimmt. In den Versuchen wird ein Probekörper unter Vorgabe einer Oberspannung $\sigma_{o}$ und einer Unterspannung $\sigma_{u}$ bis zum Bruch zyklisch belastet. Zur Beschreibung der Versuchsrandbedingungen dienen die Ober- und Unterspannung, das Spannungsverhältnis $R$, die Spannungsschwingbreite $\Delta \sigma$, die Mittelspannung $\sigma_{m}$ und die Belastungsfrequenz $f_{p}$. Die Versuche können im Druckschwell-, Zugschwell- oder Wechselbereich durchgeführt werden, vgl. Abbildung 2.15. Im Folgenden wird ausschließlich die Druckschwellbeanspruchung thematisiert.

Durch die zyklische Beanspruchung tritt das Versagen des Probekörpers bereits auf Spannungsniveaus weit unterhalb der Betondruckfestigkeit bzw. der Bruchspannung $f_{c}$ auf. Die Zahl der vom Material ertragbaren Lastwechsel, der sogenannten Bruchlastwechselzahl $N_{f}$, ist insbesondere von der Größe der Oberspannung und der Spannungsschwingbreite abhängig. Der Ermüdungswiderstand von Beton wird gewöhnlich als ertragbare Lastwechselzahl in Abhängigkeit des Oberspannungsniveaus $S_{o}=\sigma_{o} / f_{c}$ und dem Spannungsverhältnis $R$ oder dem Unterspannungsniveau $S_{u}=\sigma_{u} / f_{c}$ definiert, vgl. Model Code 2010. Die gebräuchlichste Art der Darstellung erfolgt in Form von Wöhlerlinien. Im Wöhlerkurvendiagramm wird die Bruchlastwechselzahl, meist logarithmisch skaliert, auf der Abszisse und das Oberspannungsniveau auf der Ordinate dargestellt. Das Spannungsverhältnis $R$ oder das Unterspannungsniveau $S_{u}$ dienen als Scharparameter, vgl. Abbildung 2.16.
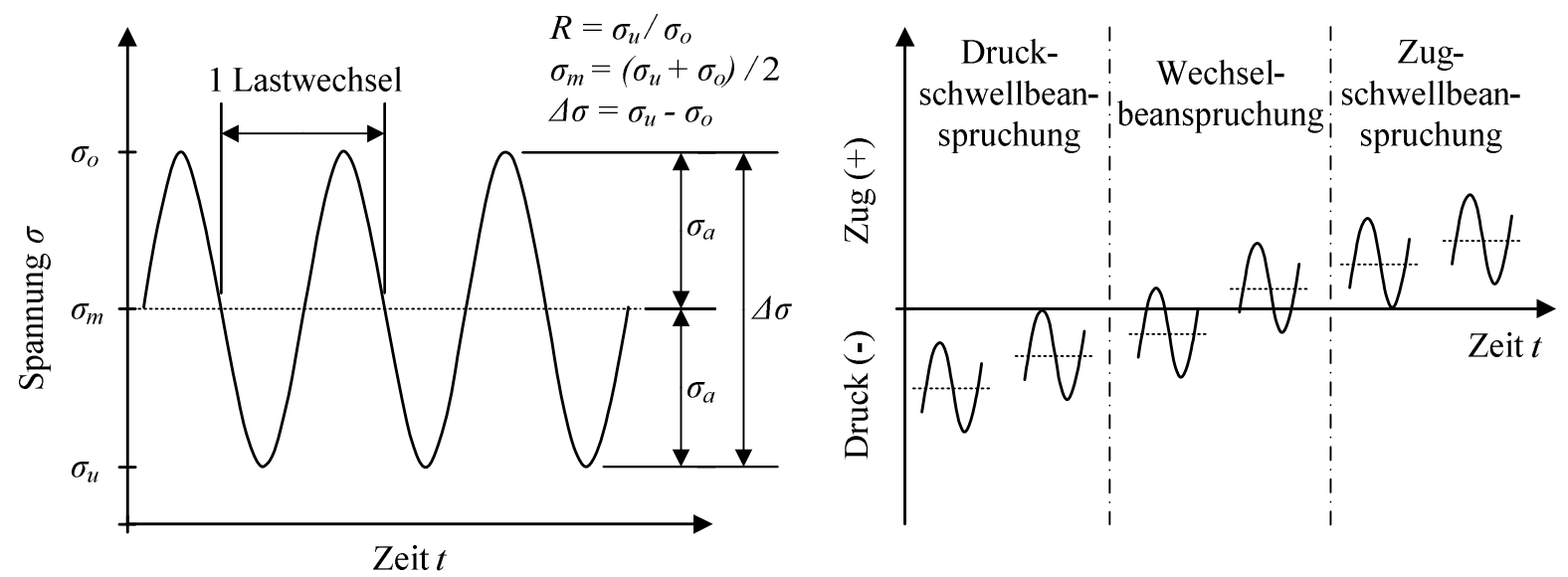

Abbildung 2.15: Kennwerte und Beanspruchungsbereiche von Ermüdungsversuchen in Anlehnung an (DIN 50100, 1978)

Der Ermüdungswiderstand von Beton ist von einer Vielzahl an Einflussfaktoren abhängig, wobei einige Faktoren eine ausschlaggebende und andere eine untergeordnete Rolle spielen. Von entscheidender Bedeutung sind die Belastungsart (Druck oder Zug, einaxial oder mehraxial, zentrisch oder exzentrisch), der Beanspruchungshorizont (Ober- und Unterspannung), die Belastungsfrequenz sowie die Prüf- und Lagerungsbedingungen der Probekörper (feucht oder 
trocken). Von untergeordneter Rolle werden die Betongüte sowie die Betonzusammensetzung (Zuschlagstoffe, Sieblinie, Zementart und Zementgehalt) erachtet. Weitere Einflussfaktoren können sich aus der Probeköpergeometrie und -größe ergeben. Einen umfassenden Einblick hinsichtlich dieser und weiterer Einflussfaktoren bieten u. a. (CEB, 1988), (König \& Danielewicz, 1994) sowie (Marx et al., 2013).

Die Auswirkungen der einzelnen Einflussfaktoren auf den Ermüdungswiderstand sind durch die Streuungen der Versuchsergebnisse meist nur schwierig zu quantifizieren. Die Streuungen resultieren u. a. aus Materialinhomogenitäten und minimalen Abweichungen in der Versuchsdurchführung. Nach (van Leeuwen \& Siemes, 1979) und (Klausen \& Weigler, 1979) können sie als logarithmisch normalverteilt angenommen werden. Zur Veranschaulichung dieser Streuungen können die Wöhlerkurvendiagramme entsprechend Abbildung 2.17 um die Versagenswahrscheinlichkeit $P$ erweitert werden. Es offenbart sich, dass die Streuungen mit abnehmendem Oberspannungsniveau zunehmen.

Ein weiteres Einteilungskriterium kann entsprechend des Belastungsszenarios vorgenommen werden. In den zuvor beschriebenen einstufigen Ermüdungsuntersuchungen sind Ober- und Unterspannung bis zum Versagen des Probekörpers konstant. In mehrstufigen Untersuchungen können diese während der Prüfdauer variieren oder Belastungsszenarios berücksichtigt werden, um Belastungsänderungen, Reihenfolgeeffekte oder auch den Einfluss von Belastungspausen zu untersuchen.

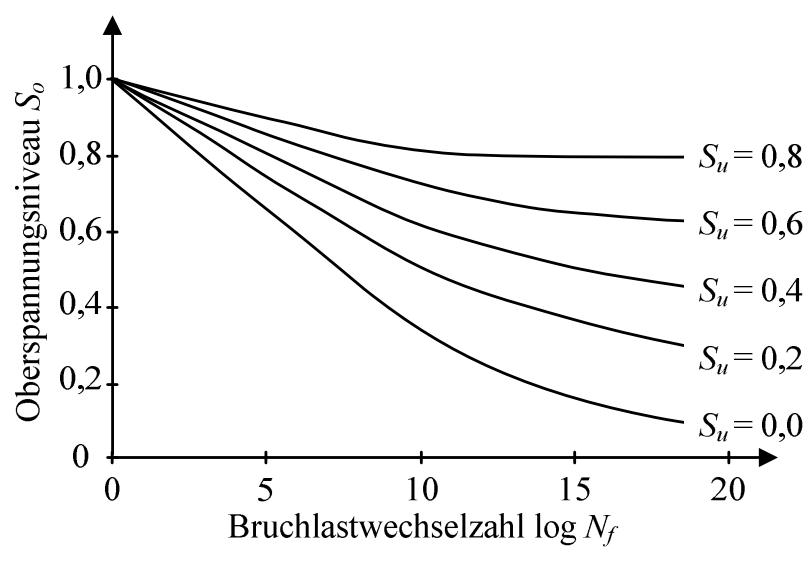

Abbildung 2.16: Wöhlerlinien für ermüdungsbeanspruchtem Beton nach Model Code 2010

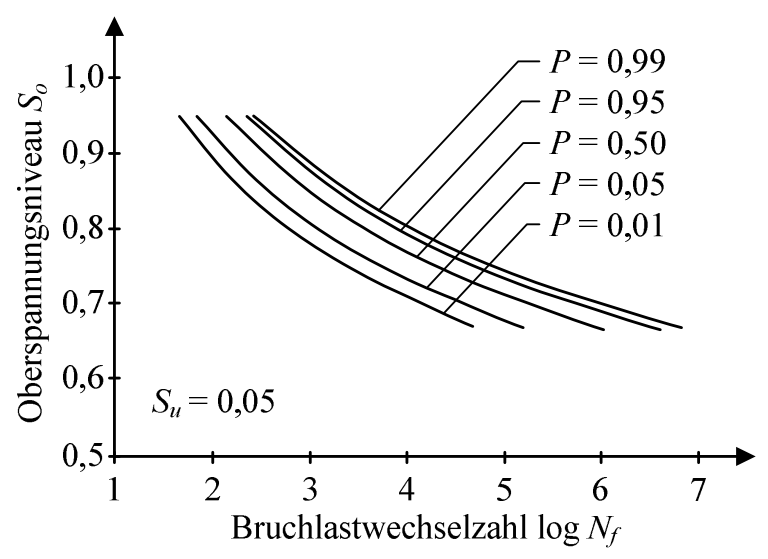

Abbildung 2.17: Wöhlerlinien mit unterschiedlicher Versagenswahrscheinlichkeiten nach (Holmen, 1979)

In Abhängigkeit von der erreichten Bruchlastwechselzahl können Ermüdungsversuche in niederzyklische (engl.: Low-Cycle-Fatigue), hochzyklische (engl.: High-Cycle-Fatigue) und sehr hochzyklische (engl.: Very-High-Cycle-Fatigue) Untersuchungen unterteilt werden. Die Grenzen sind in der Literatur nicht eindeutig gezogen, üblicherweise werden sie bei $10^{3}$ und $10^{7}$ Lastwechseln angenommen, vgl. (Hsu, 1981; König \& Danielewicz, 1994). Der Großteil der in der Literatur dokumentierten Ermüdungsversuche sind dem niederzyklischen und hochzyklischen Bereich zuzuordnen. 


\subsubsection{Phänomenologisches Materialverhalten}

Während des Ermüdungsversuchs finden im Beton Schädigungsprozesse statt, die sich auf der Mesoskala in Form von Rissen manifestieren. Die Schädigungsprozesse können in drei charakteristische Phasen gegliedert werden, die im Folgenden erläutert werden. Der Phasenübergang von Phase I zu Phase II liegt üblicherweise bei etwa $10 \%$ bis $20 \%$ und der Phasenübergang von Phase II zu Phase III bei $80 \%$ bis $90 \%$ der ertragbaren Bruchlastwechselzahl. Die erste und dritte Phase sind bei normalfesten Betonen in der Regel etwas länger und ausgeprägter als bei hochfesten und ultrahochfesten Betonen.

In der ersten Phase findet eine rasante Mikrorissbildung statt. Die Mikrorisse entstehen neu oder die im Zementstein vorhandenen Schwindrisse wachsen an. In der folgenden Phase findet ein stabiles und kontinuierliches Mikrorisswachstum statt. In der dritten und letzten Phase vereinigen sich diese Mikrorisse und es kommt zur Ausbildung von Bruchflächen und schließlich zum Versagen der Probe. Die Rissentwicklung konnte hinsichtlich Mikrorissanzahl, -breite und -fläche u. a. anhand mikroskopischer Untersuchungen nachgewiesen werden (Reinhardt et al., 1978; Breitenbücher et al., 2008). Aber auch Schallemissionsanalysen an zyklisch beanspruchten Versuchskörpern spiegeln die drei Phasen der Rissentwicklung wider (Thiele et al., 2011; Urban et al., 2014). Nach einer anfänglichen starken Schallemittierung nehmen die Schallsignale entsprechend des stabilen Rissfortschritts ab, während sie in der letzten Phase wieder deutlich zunehmen. Die Ergebnisse von Ultraschalluntersuchungen an zyklisch beanspruchten Betonproben veranschaulichen ebenfalls die drei Phasen der Rissentwicklung, vgl. Abbildung 2.18 .

Im Vergleich zur monoton steigenden Druckbeanspruchung führen Ermüdungsbeanspruchungen zu einer feineren Rissverteilung und Rissdichte (Maher \& Darwin, 1982; König \& Danielewicz, 1994). Diese These wird von Ultraschalluntersuchungen in (von der Haar et al., 2015b) und (von der Haar \& Marx, 2016a) bestätigt.

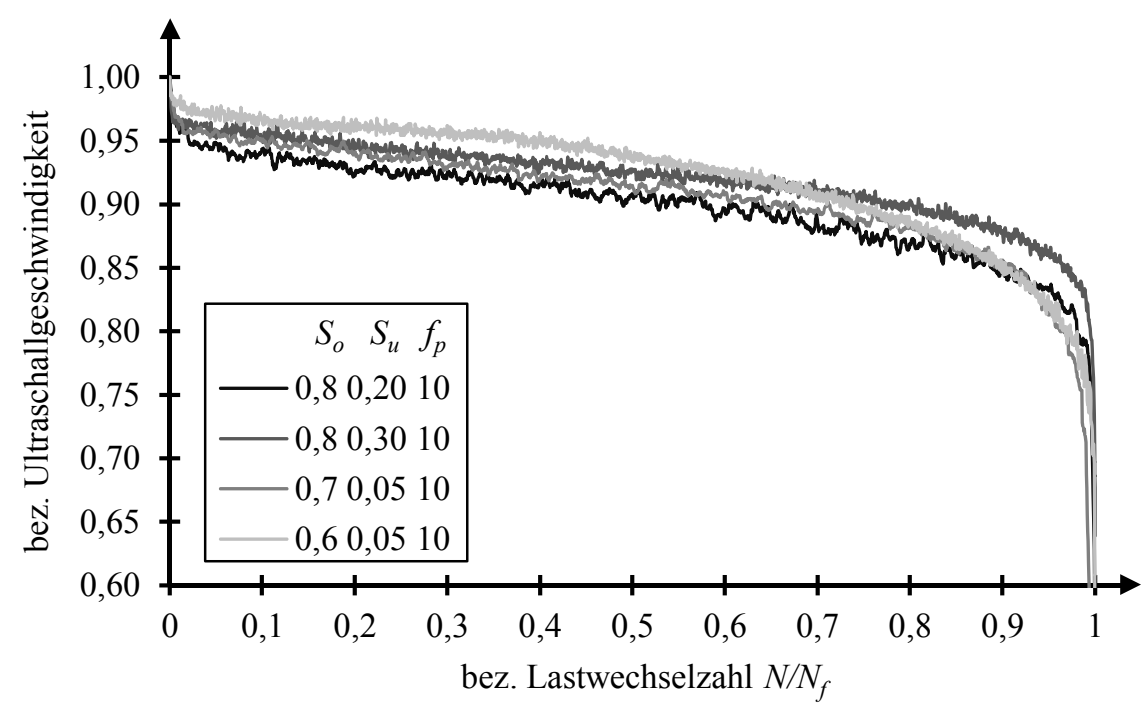

Abbildung 2.18: Entwicklung der Ultraschallgeschwindigkeit in Abhängigkeit von der Lastwechselzahl (von der Haar \& Marx, 2016b) 
Makroskopisch stellt sich das Materialverhalten von Beton unter Druckschwellbeanspruchung als dreiphasige Dehnungsevolution dar, vgl. Abbildung 2.19. Die erste Phase ist durch eine abnehmende Steigung des Dehnungsverlaufs, die zweite durch eine konstante und die dritte durch eine zunehmende Steigung gekennzeichnet. (Awad \& Hilsdorf, 1971) stellten in niederzyklischen Ermüdungsuntersuchungen fest, dass das Querdehnungsverhältnis in der Phase des instabilen Risswachstums überproportional zunimmt, während es in der ersten und zweiten Phase näherungsweise konstant ist.

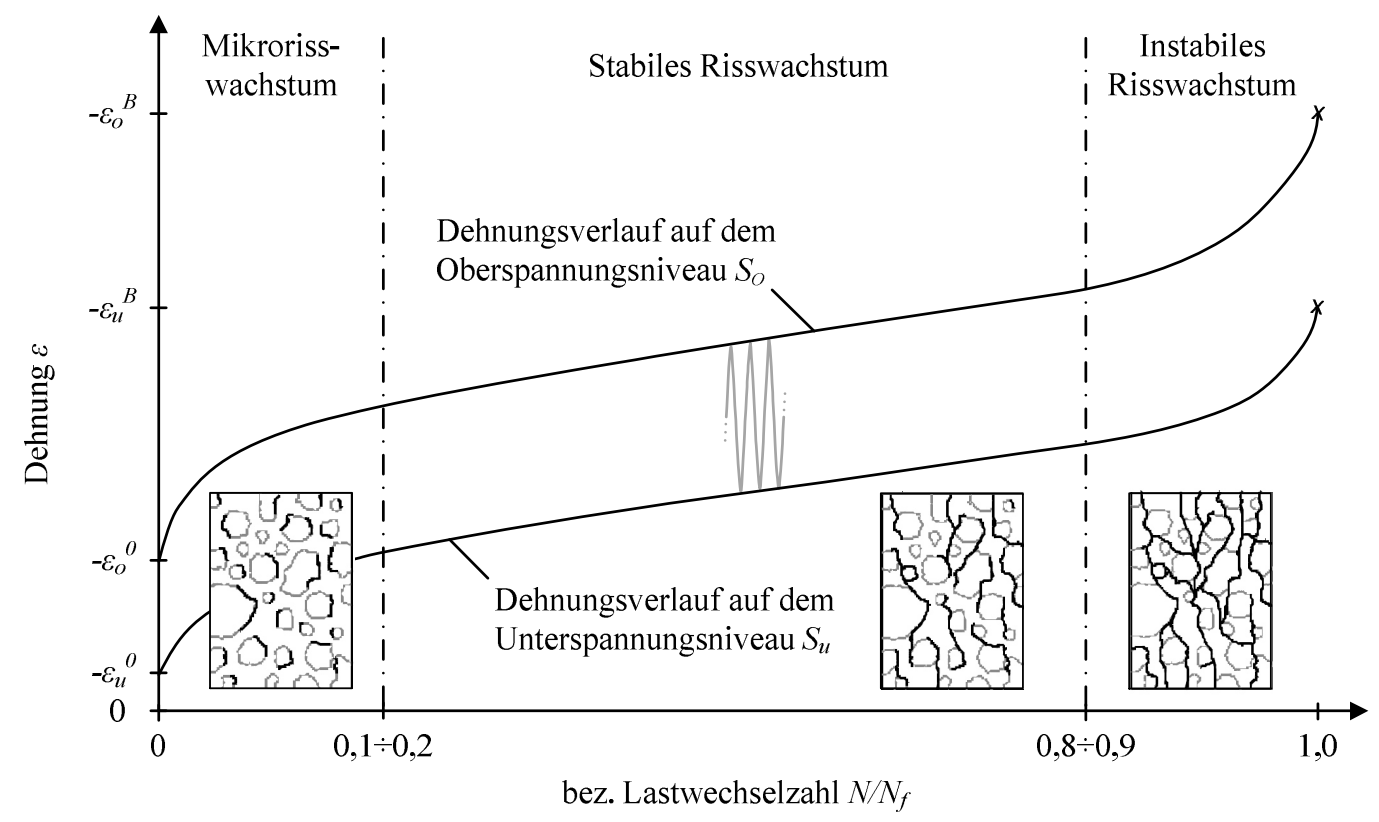

Abbildung 2.19: Dehnungsentwicklung mit schematischer Darstellung der Rissbildung nach (Göhlmann, 2009)

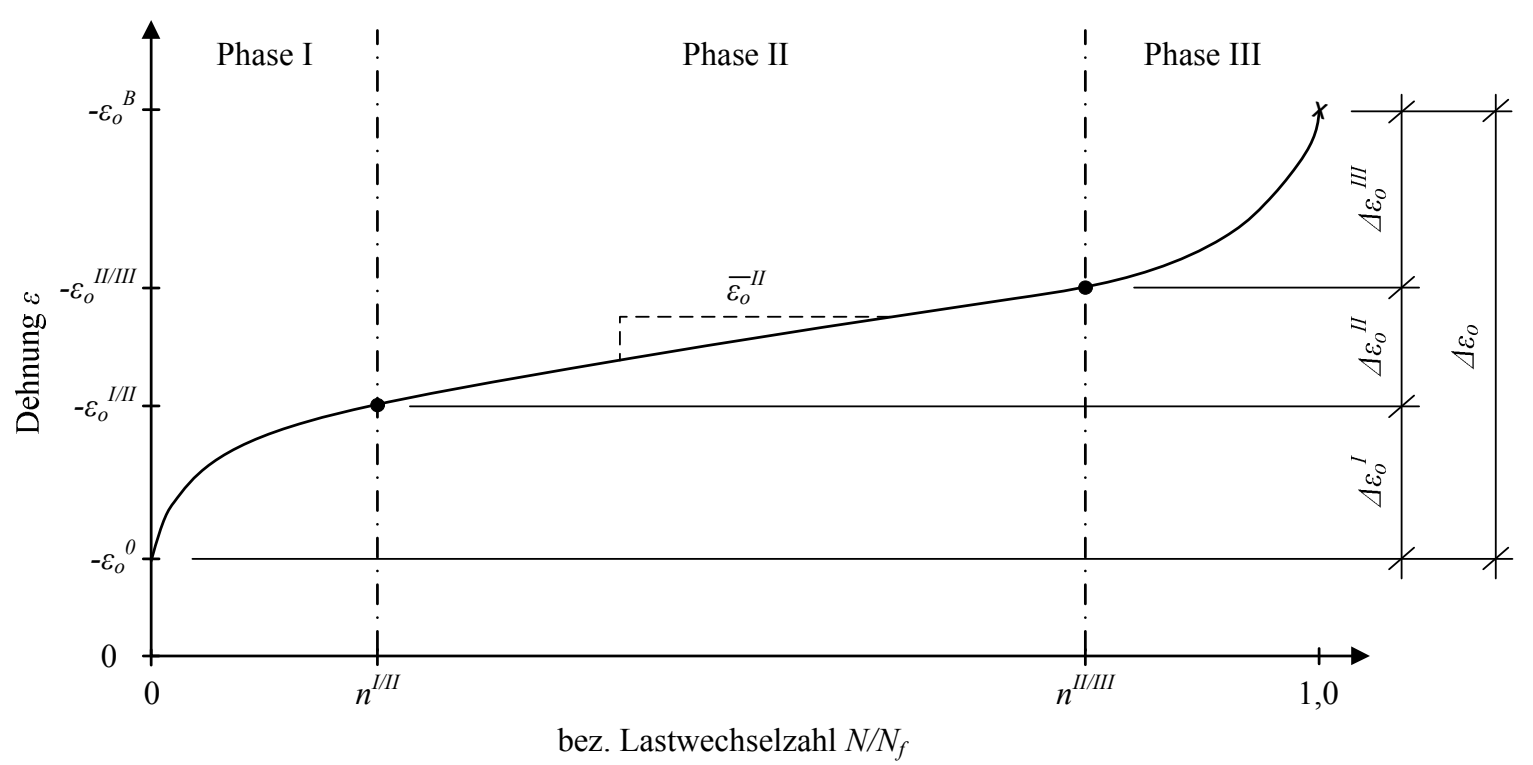

Abbildung 2.20: Parameterdefinition für den Dehnungsverlauf auf dem Oberspannungsniveau 
Die Abbildung 2.20 veranschaulicht die im Zusammenhang mit der Dehnungsentwicklung unter Druckschwellbeanspruchung festgelegte Parameterdefinition. Dargestellt ist die Definition für das Oberspannungsniveau. Die Parameter des Unterspannungsniveaus sind im Fußzeiger durch ein u anstelle eines o's gekennzeichnet, vgl. Abbildung 2.19.

Im Rahmen von Versuchsdatenauswertungen erkannten (Sparks \& Menzies, 1973) einen stringenten, linearen Zusammenhang zwischen der Steigung des Dehnungsverlaufs in der Phase des stabilen Risswachstums $\left(\bar{\varepsilon}_{o}^{-I I}\right.$ bzw. $\left.\varepsilon_{u}\right)$ mit der Bruchlastwechselzahl im doppelt logarithmischen Maßstab. Dieser lineare Zusammenhang konnte im Zuge weiterer Arbeiten für unterschiedliche Betone und Versuchsrandbedingungen bestätigt werden (Do et al., 1993; Wefer, 2010; Oneschkow, 2014). Die Auswertungen zeigen, dass die Steigungen auf dem Unterspannungsniveau kleiner sind als auf dem Oberspannungsniveau. Diese Differenz ist auf die Reduzierung der Probekörpersteifigkeit während der Versuchsdurchführung zurückzuführen. Vergleichende Auswertungen zur Steigung des Dehnungsverlaufs in der zweiten Phase an normalfesten und hochfesten Betonen zeigen, dass die Steigung von hochfesten Betonen kleiner ist als von normalfesten Betonen (Petkovic et al., 1992; Do et al., 1993).

\section{Steifigkeitsentwicklung}

Mit zunehmender Schädigung nimmt die Steifigkeit des Betons im Ermüdungsversuch ab. Die Entwicklung der Probekörpersteifigkeit wird in der Regel über den Sekantenmodul $E_{s}$ beschrieben (Holmen, 1979; Oneschkow, 2014). Dieser wird aus den aufgezeichneten Dehnungsverläufen entsprechend Gleichung 2.29 für den Entlastungsast der einzelnen Lastzyklen ermittelt. Die Auswertung des Sekantenmoduls für sämtliche Lastwechsel des Ermüdungsversuchs liefert den Verlauf des Sekantenmoduls über die Versuchsdauer.

$$
E_{s}=\frac{\sigma_{o}-\sigma_{u}}{\varepsilon_{o}-\varepsilon_{u}}
$$

Der Sekantenmodul $E_{s}$ entwickelt sich reziprok zum S-förmigen Dehnungsverlauf, vgl. Abbildung 2.21. Untersuchungen von (Holmen, 1979) an einem normalfesten Beton $\left(f_{c, \text { cube, } 28 d} \approx 40 \mathrm{~N} / \mathrm{mm}^{2}\right)$ zeigen, dass der Sekantenmodul bis zum Bruch auf etwa $60 \%$ des anfänglichen Steifigkeitswertes abnimmt. Untersuchungen von (Do et al., 1993) an zwei hochfesten Betonen $\left(f_{c, c y l, 28 d}=68,9 \mathrm{~N} / \mathrm{mm}^{2}\right.$ und $\left.f_{c, c y l, 28 d}=94,1 \mathrm{~N} / \mathrm{mm}^{2}\right)$ stellen Steifigkeitsabnahmen auf $75 \%$ bis $85 \%$ und $85 \%$ bis $95 \%$ der Anfangssteifigkeit fest. Der höherfestere Beton wies die geringere Steifigkeitsabnahme auf. Versuche von (Oneschkow, 2014) an einem hochfesten Beton $\left(f_{c, \text { cube } 28 d}=116 \mathrm{~N} / \mathrm{mm}^{2}\right)$ offenbaren Steifigkeitsabnahmen auf ca. $85 \%$ der Anfangssteifigkeit. Die Literaturauswertung lässt vermuten, dass die Steifigkeitsabnahme bis zum Bruch mit zunehmender Betonfestigkeit abnimmt, sich der höherfeste Beton also analog zum Verhalten unter monoton steigender Beanspruchung weniger duktil verhält. Im Weiteren kommen (Holmen, 1979) und (Do et al., 1993) zu dem Ergebnis, dass zwar der Verlauf der Steifigkeitsabnahme bis zum Bruch vom Oberspannungsniveau beeinflusst wird, die Gesamtsteifigkeitsabnahme aber unabhängig vom Oberspannungsniveau ist, vgl. Abbildung 2.21. Das Versagen tritt somit bei 
Erreichen einer bestimmten Steifigkeitsdegradation bzw. Materialschädigung auf. Demgegenüber zeigen Untersuchungen von (Oneschkow, 2016) und (von der Haar \& Marx, 2016b), dass die Gesamtsteifigkeitsänderung mit abnehmendem Oberspannungsniveau zunimmt.

In (Jinawath, 1974) wurden Ermüdungsversuche nach dem Erreichen gewisser Lastwechselzahlen auf dem Unterspannungsniveau gestoppt und monoton bis auf das Oberspannungsniveau belastet und wieder entlastet. Die Spannungsdehnungslinien wurden für diese Be- und Entlastungsphasen aufgezeichnet und in Abbildung 2.22 dargestellt. Anschließend wurden die Ermüdungsversuche fortgesetzt. In den Versuchen konnte nicht nur eine Steifigkeitsreduktion festgestellt werden, sondern mit zunehmender Lastzyklenzahl änderte sich auch die Form der Be- und Entlastungspfade. Zu Beginn der Ermüdungsversuche waren die Belastungsäste zunächst konkav zur Dehnungsachse gekrümmt. Nach nur wenigen Lastzyklen nahm der Belastungsast eine gerade und nach weiteren Lastzyklen eine konvexe Form an. In Untersuchungen von (Mehmel \& Kern, 1962) erfolgte die Krümmungsumkehr umso schneller, je höher das Oberspannungsniveau war. Das Bemerkenswerte daran ist, dass im unteren Spannungsbereich eine Steifigkeitsreduzierung, im oberen Spannungsbereich aber eine Steifigkeitserhöhung festzustellen ist. Der Entlastungsast ist von Beginn an konvex zur Dehnungsachse gekrümmt und ändert seinen grundsätzlichen Verlauf nicht. Wie in Abbildung 2.22 ersichtlich, nimmt der Flächeninhalt zwischen den einzelnen Be- und Entlastungspfaden mit zunehmender Lastwechselzahl ab. Untersuchungen u. a. von (Ban, 1933), (Gaede, 1962), (Mehmel \& Kern, 1962) und (Ibuk, 2008) bestätigen diese Ergebnisse.

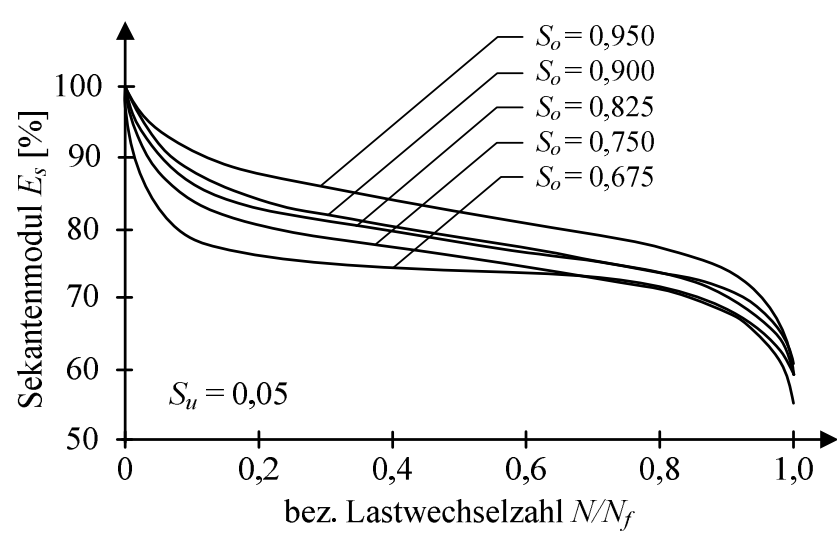

Abbildung 2.21: Entwicklung des Sekantenmoduls nach (Holmen, 1979)

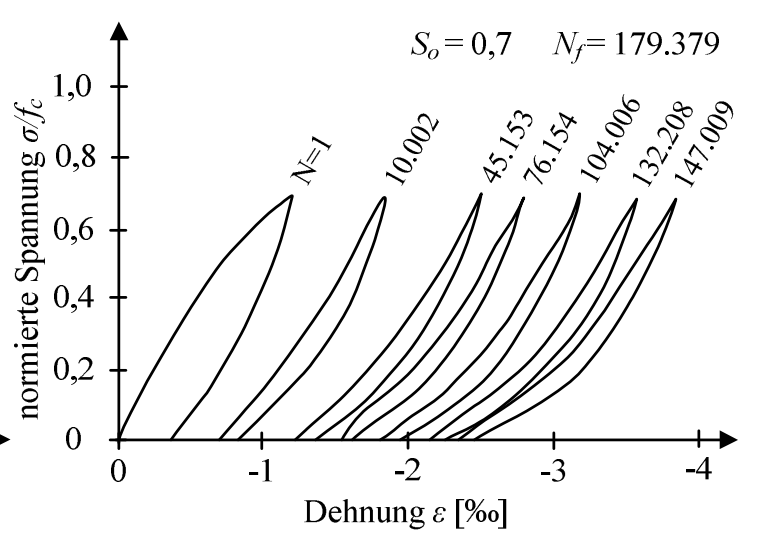

Abbildung 2.22: Zyklische Spannungsdehnungsbeziehung nach (Jinawath, 1974)

Die Verformungen von heterogenen Stoffen wie Beton werden durch die kontinuierliche Phase, also dem Zementstein, bestimmt (Freudenthal, 1955). Die Belastungsäste von viskosen Stoffen wie dem Zementstein weisen typischerweise eine zur Dehnungsachse gekrümmte Form auf, so auch die des Betons während der ersten Lastzyklen. Diese ersten Zyklen bewirken bereits plastische Verformungen des Zementsteins. Die Zuschlagstoffe werden ineinander verschoben und der Zementstein, der in Kraftrichtung gesehen zwischen diesen liegt, wird verdichtet. Die Gesteinskörner und der verdichtete Zementstein werden zu einer kontinuierlichen Phase und blockieren das viskose Verhalten. Diese Verdichtung setzt sich im Laufe des Ermüdungsversuchs 
fort. Diese über den Betonkörper ungleichmäßig verteilten plastischen Stauchungen rufen bei Entlastung Eigenspannungen hervor. Die Größe dieser Eigenspannungen werden von dem Maß der verbleibenden relativen Elastizität des Zementsteins bestimmt. Überschreiten sie die Haftzugfestigkeit zwischen Zementstein und Zuschlagstoffen, führen sie zu Mikrorissen. Bei der Wiederbelastung überlagern sich Eigenspannungen und die Spannungen aus äußerer Last. Mit zunehmender Belastung schließen sich zunächst die Risse. Dies führt im unteren Spannungsbereich zu der o. g. Steifigkeitsreduktion. Nach dem Schließen der Risse beteiligt sich die verdichtete, kontinuierliche Phase wieder am Lastabtrag. Dies führt zur Steifigkeitserhöhung im oberen Spannungsbereich (Mehmel \& Kern, 1962). Durch diesen Prozess tritt zusätzlich zu den Rissen aus Querzug (vgl. Abbildung 2.2) eine Rissbildung senkrecht zur Kraftrichtung auf. (Ibuk, 2008) konnte die Risse aus Querzug und senkrecht zur Kraftrichtung mikroskopisch nachweisen.

In (Breitenbücher et al., 2007), (Ibuk, 2008) und (Urban et al., 2014) wurde die Steifigkeitsentwicklung mittels Ultraschallmessungen untersucht. Die Rissbildung im Beton führt zu einer Vergrößerung der Ultraschalllaufzeit. Im Grundsatz spiegelt sich der S-förmige Verlauf über die Versuchsdauer wider. Über diese Laufzeitänderung kann aber nicht unmittelbar auf die Änderung der Sekantensteifigkeit geschlossen werden (von der Haar \& Marx, 2016b).

\section{Ermüdungsbruchdehnung}

In Bezug auf das Verformungsverhalten spielt die Bruchdehnung im Ermüdungsversuch eine maßgebende Rolle. Um Verwechslungen mit der Bruchdehnung unter monoton steigender Beanspruchung $\varepsilon_{c} \quad \mathrm{zu}$ vermeiden, wird die Bruchdehnung im Ermüdungsversuch als Ermüdungsbruchdehnung bezeichnet.

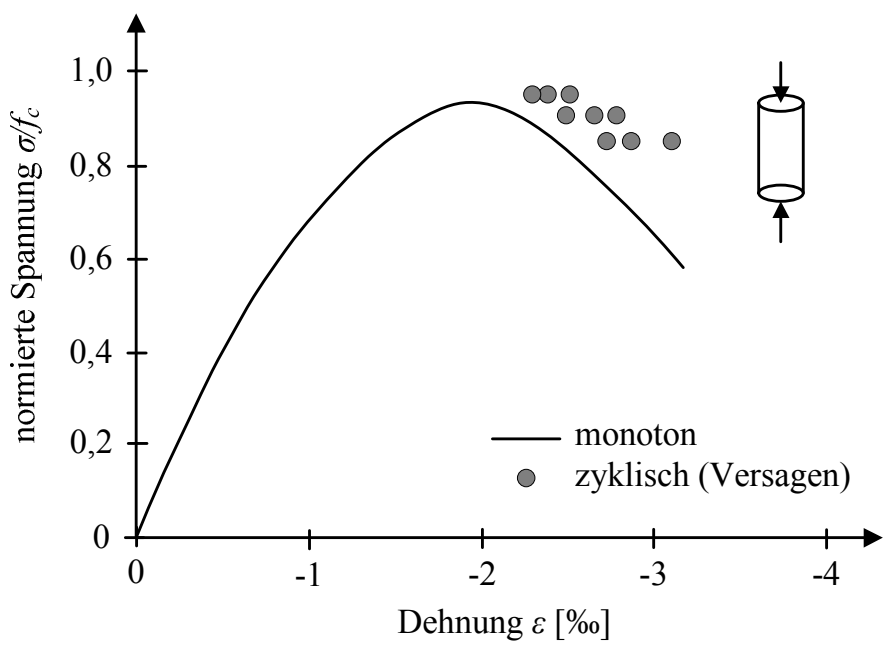

Abbildung 2.23: Dehnungsverlauf unter monoton steigender Beanspruchung und Ermüdungsbruchdehnungen bei zyklischer Beanspruchung aus Versuchen von (Awad \& Hilsdorf, 1971) aus (Pfanner, 2003)

(Awad \& Hilsdorf, 1971) führten Ermüdungsversuche an einem normalfesten Beton auf hohen Oberspannungsniveaus durch. Die Untersuchungen wurden in erster Linie zur Validierung bzw. Beurteilung bestehender Envelope-Konzepte (vgl. Abschnitt 2.3) vollzogen. Während die 
Spannungsdehnungslinie des Betons innerhalb weniger Minuten aufgezeichnet wurde, dauerten die Ermüdungsversuche teilweise mehrere Stunden. Es zeigte sich, dass die Ermüdungsbruchdehnungen in der Regel außerhalb der monotonen Spannungsdehnungslinie lagen, vgl. Abbildung 2.23. Zudem konnte festgestellt werden, dass die Ermüdungsbruchdehnungen mit zunehmenden Versuchslaufzeiten, durch Reduzierung der Belastungsfrequenz oder der Spannungsschwingbreite (abnehmendes Oberspannungsniveau bzw. zunehmendes Unterspannungsniveau), zunahmen.

(Shah et al., 1983) führten Ermüdungsversuche $\left(S_{o}=0,80-0,95 ; S_{u}=0,10-0,50\right)$ an unbewehrten und wendelbewehrten Probekörpern durch. Sie stellten fest, dass bei niedrigen Belastungsfrequenzen und hohen Bruchlastwechselzahlen die Ermüdungsbruchdehnungen die monotone Spannungsdehnungslinie übertrafen. Andernfalls versagten die Probekörper meist bei Erreichen der Envelope-Kurve. Da die Autoren die monotone Spannungsdehnungslinie als Grenzlinie für die elastischen und die infolge Schädigung eintretenden plastischen Verformungen ansahen, schlussfolgerten sie, dass sich die Ermüdungsbruchdehnungen aus zeitabhängigen sowie elastischen und schädigungsinduzierten Dehnungsanteilen zusammensetzen.

Die Abbildung 2.24 stellt die Ermüdungsbruchdehnungen $\left(S_{o}=0,675-0,95 ; S_{u}=0,05-0,20 ; f_{p}=\right.$ $1-10 \mathrm{~Hz})$ eines normalfesten Betons $\left(f_{c, \text { cube,28d }} \approx 40 \mathrm{~N} / \mathrm{mm}^{2}\right)$ aus (Holmen, 1979) dar. Begleitend zu den Ermüdungsuntersuchungen führte Holmen kraftgeregelte Druckfestigkeitsversuche an dem gleichen Beton und der gleichen Probekörpergeometrie, wie für die Ermüdungsuntersuchungen verwendet, durch. Die Bruchdehnungen dieser Versuche lagen im Mittel bei $\varepsilon_{c}=-2,78 \%$ und sind ebenfalls in der Abbildung dargestellt. Es zeigt sich, dass die Ermüdungsbruchdehnungen größer sind als die Bruchdehnungen unter monoton steigender Beanspruchung und mit abnehmendem Oberspannungsniveau tendenziell zunehmen.

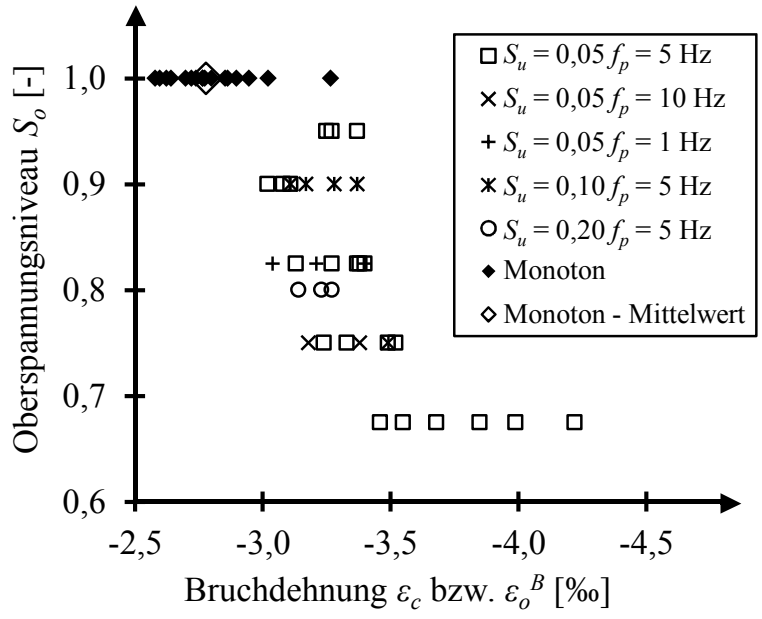

Abbildung 2.24: Bruchdehnungen und Ermüdungsbruchdehnungen aus (Holmen, 1979)

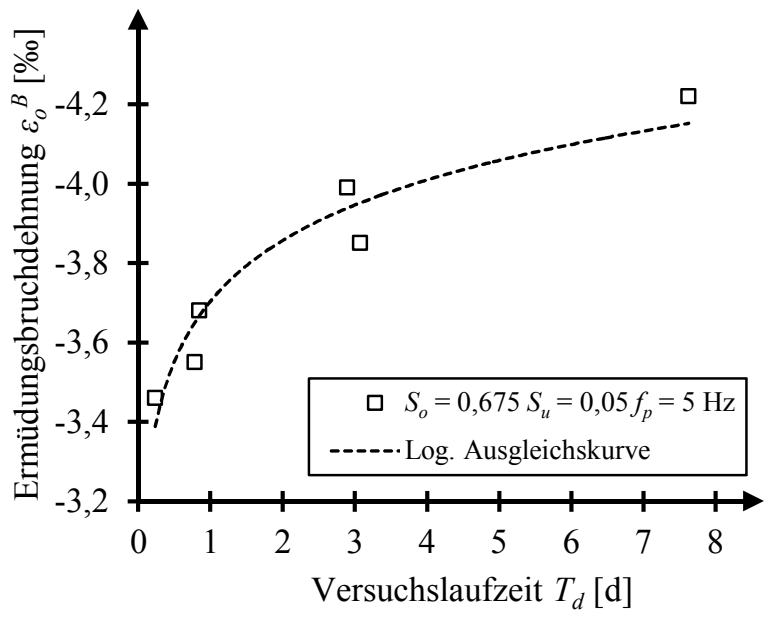

Abbildung 2.25: Ermüdungsbruchdehnung in Abhängigkeit der Zeit aus (Holmen, 1979)

Werden die Betondehnungen und die zugehörigen Bruchlastwechselzahlen aus (Holmen, 1979) auf dem geringsten Oberspannungsniveau von $S_{o}=0,675$ im Detail betrachtet, so ergeben sich Bruchlastwechselzahlen zwischen $N_{f}=102.950$ und 3.294.820. Bei der von Holmen gewählten 
Belastungsfrequenz von $5 \mathrm{~Hz}$ lagen die Versuchslaufzeiten damit zwischen $T_{d}=0,24$ Tagen und 7,63 Tagen. Die Differenz zwischen minimaler und maximaler Ermüdungsbruchdehnung betrug $0,8 \%$. In Abbildung 2.25 sind die Ermüdungsbruchdehnungen über die Versuchsdauer aufgetragen. Da die Ermüdungsbruchdehnungen mit der Versuchsdauer zunehmen, spricht dies für die These, dass die Differenz der Ermüdungsbruchdehnungen nicht allein aus den Streuungen des Materials, sondern auch aus dem zeitabhängigen Verformungsverhalten des Betons resultiert. So stellte auch (Bazant, 1968) die These auf, dass sich die Verformungen im Ermüdungsversuch zyklen- als auch zeitabhängig entwickeln.

(Do et al., 1993) stellten in Ermüdungsuntersuchungen $\left(S_{o}=0,70-0,95 ; S_{u}=0,05 ; f_{p}=1 \mathrm{~Hz}\right)$ an zwei hochfesten Betonen $\left(f_{c, c y l, 28 d}=68,9 \mathrm{~N} / \mathrm{mm}^{2}\right.$ und $\left.f_{c, c y l, 28 d}=94,1 \mathrm{~N} / \mathrm{mm}^{2}\right)$ fest, dass die Ermüdungsbruchdehnungen in der Größenordnung der Bruchdehnung unter monoton steigender Beanspruchung lagen bzw. unwesentlich größer waren. Auf Grundlage der Darstellungen kann jedoch nicht geschlossen werden, ob das Oberspannungsniveau, die Versuchsdauer oder die Betonfestigkeitsklasse einen Einfluss auf die Ermüdungsbruchdehnung hatten.

(Kono et al., 2008) untersuchten drei hochfeste bis ultrahochfeste Betone $\left(f_{c, c y l}=100 \mathrm{~N} / \mathrm{mm}^{2}\right.$ $\left.170 \mathrm{~N} / \mathrm{mm}^{2}\right)$ unter zyklischer Beanspruchung $\left(S_{o}=0,70-0,90 ; S_{u}=0,10 ; f_{p}=0,2-0,5 \mathrm{~Hz}\right)$. Das Durchläuferniveau der Versuche wurde zu 200 Lastwechseln gewählt. Daraus resultiert eine maximale Versuchsdauer von rd. 17 min. Die Probekörper, die innerhalb dieser 200 Lastwechsel versagten, wiesen Oberspannungsniveaus von $S_{o} \geq 0,80$ auf. Die Ermüdungsbruchdehnungen lagen bei $90 \%$ bis $100 \%$ der mittleren Bruchdehnung unter monoton steigender Beanspruchung.

Die Versuchsergebnisse von (Oneschkow, 2014) $\left(S_{o}=0,80-0,90 ; S_{u}=0,05 ; f_{p}=0,1-10 \mathrm{~Hz}\right)$ an einem hochfesten Beton $\left(f_{c, \text { cube,28d }}=116 \mathrm{~N} / \mathrm{mm}^{2}\right)$ weisen ebenfalls Ermüdungsbruchdehnungen unterhalb der Bruchdehnung bei monoton steigender Beanspruchung auf. Zudem wird die Wahrscheinlichkeit für das Unterschreiten der Bruchdehnung mit $70 \%$ bis $100 \%$ angegeben. Der Großteil der Versuche nahm eine Versuchsdauer von weniger als eine Stunde in Anspruch.

Die Literaturauswertung zeigt, dass die Ermüdungsbruchdehnungen vom zeitabhängigen Verformungsverhalten des Betons beeinflusst werden. So weisen langandauernde Versuche größere Ermüdungsbruchdehnungen auf als kürzere, wie insbesondere Abbildung 2.25 zeigt. Andererseits weisen normalfeste Betone auch auf hohen Beanspruchungsniveaus und bei dementsprechend kurzen Versuchsdauern Ermüdungsbruchdehnungen auf, die tendenziell größer sind als die Bruchdehnung unter monoton steigender Beanspruchung. Demgegenüber liegen die Ermüdungsbruchdehnungen hochfester Betone in der Größenordnung der Bruchdehnung oder unterschreiten diese. Diese Beobachtung steht im Einklang mit dem Nachbruchverhalten normalfester und hochfester Betone. Hochfeste Betone weisen einen deutlich steileren Abfall der Spannungsdehnungslinie nach Überschreiten der Höchstlast auf als normalfeste Betone. Die Duktilität des Betons nimmt also mit zunehmender Betonfestigkeit ab. Diese Eigenschaft scheint sich in den Ermüdungsbruchdehnungen widerzuspiegeln. Im Weiteren treten bei hochfesten Betonen durch die geringere Porosität des Zementsteins kleinere Kriechverformungen auf als bei normalfesten Betonen unter einer dauerhaften Druckbeanspruchung (Tue \& Mucha, 2006; Zilch 
\& Zehetmaier, 2010). Dementsprechend sind bei hochfesten Betonen kleinere zeitabhängige Verformungen im Ermüdungsversuch zu erwarten als bei einem normalfesten Beton. Diese Beobachtungen lassen einen Zusammenhang zwischen den Ermüdungsbruchdehnungen, dem Kriechverhalten und dem Dehnungsverhalten unter monoton steigender Beanspruchung erwarten.

\section{Zusammenhang zwischen Kriechdehnung und der Dehnungsentwicklung im Ermüdungsversuch}

Die Kriechverformungen unter einer dauerhaften, konstanten Druckbeanspruchung sind von der Belastungsdauer und der Beanspruchungshöhe abhängig, während der Ermüdungsversuch, und damit auch die Verformungen der Betonkörper, durch Mittelspannung, Spannungsschwingbreite, Lastwechselzahl und Belastungsfrequenz charakterisiert sind. Lastwechselzahl und Belastungsfrequenz sind mit der Belastungsdauer über die Beziehung $t=N / f_{p}$ verknüpft. Ein Ansatz zur Verknüpfung von Kriechspannung, Mittelspannung und Spannungsschwingbreite ist nicht bekannt. Nur für den Sonderfall, dass die Spannungsschwingbreite gleich null ist $(\Delta \sigma=0)$, entspricht die Mittelspannung der Kriechspannung $\left(\sigma_{m}=\sigma_{c r}\right)$, in diesem Fall gleicht der Beanspruchungsverlauf des Ermüdungsversuchs dem des Kriechversuchs.

(Mehmel \& Kern, 1962) führten Kriechversuche auf dem Oberspannungsniveau von Ermüdungsversuchen $\left(S_{o}=0,40-0,80 ; R=0,15-0,90 ; f_{p} \approx 6 \mathrm{~Hz}\right)$ durch. Die Dehnungen in den Kriechversuchen waren durchweg größer als die auftretenden Dehnungen auf dem Oberspannungsniveau der Ermüdungsversuche. Die Differenzen zwischen den Verformungsverläufen waren jedoch gering. Die Unterspannung wies in den Untersuchungen keinen nennenswerten Einfluss auf die Verformung auf. Daraus wurde geschlussfolgert, dass sich die Verformungen infolge zyklischer und dauerhafter Beanspruchung durch die gleiche mathematische Funktion beschreiben lassen, wenn die Oberspannung des zyklischen Versuchs der Beanspruchung im Kriechversuch entspricht. Demgegenüber ergaben zyklische Untersuchungen von (Gaede, 1962), dass größere Spannungsschwingbreiten zu einem stärkeren Wachstum der plastischen Verformungen im Ermüdungsversuch führen als kleinere Spannungsschwingbreiten. In (Plähn, 1969) wird der widersprüchliche Einfluss der Unterspannung auf die Versuchsergebnisse von Mehmel \& Kern und Gaede diskutiert. Es wird geschlossen, dass der vernachlässigbare Einfluss der Unterspannung in Mehmel \& Kern versuchstechnisch zu begründen ist. Durch die eingesetzte Prüfmaschine waren Vor- und Zwischenbelastungen auf hohen Beanspruchungsniveaus notwendig, die zu unbeabsichtigten Verformungen und damit zur Verfälschung der Ergebnisse geführt haben dürften. Die bleibenden Verformungen dürften daher insbesondere bei niedrigen Unterspannungen im Vergleich $\mathrm{zu}$ einer reinen Schwingbeanspruchung zu groß gewesen sein, wodurch der Einfluss der Unterspannung unterschätzt wurde.

An einem Leichtbeton untersuchten (Rostásy et al., 1975) das Verformungsverhalten von dauerhaft und zyklisch beanspruchten Betonproben. Die zyklisch belasteten Proben wurden bei einer mittleren Belastungsfrequenz von 15 Lastwechseln pro Tag mit insgesamt 1.200 bis 2.000 
Lastwechseln belastet. Das Kriechspannungsniveau entsprach dem Oberspannungsniveau der zyklischen Beanspruchung. Das Oberspannungsniveau lag bei $25 \%$ der Betondruckfestigkeit und das Spannungsverhältnis wurde zu $R=0,3$ gewählt. Das Beanspruchungsniveau der Betonproben lag somit in einem Bereich, in dem von einem linear-elastischen Spannungsdehnungsverhalten und vom linearen Kriechen ausgegangen werden kann. Analog zu den Ergebnissen von (Mehmel \& Kern, 1962) und (Gaede, 1962) übertrafen die Kriechdehnungen die Dehnungen auf dem Oberspannungsniveau der zyklischen Versuche. Als Ursache wird die andauernde Wirkung der Kriechspannung gegenüber dem zyklischen Beanspruchungsverlauf genannt. Im Weiteren stellten die Autoren fest, dass auf dem Mittelspannungsniveau des zyklischen Versuchs ungefähr die gleichen Verformungen auftraten wie im Kriechversuch, wenn dieser auf dem Mittelspannungsniveau des zyklischen Versuchs durchgeführt wird. Die Autoren gingen davon aus, dass die Belastungsfrequenz keinen Einfluss auf die zeitabhängigen Verformungen hat.

(Whaley \& Neville, 1973) proklamieren auf Grundlage von Ermüdungs- und Kriechversuchen, dass sich die zeitabhängigen, viskosen Dehnungen im Ermüdungsversuch aus Kriechdehnungen, die unter einer dauerhaften Last auf dem Mittelspannungsniveau des Ermüdungsversuchs auftreten, und einem von der Spannungsamplitude abhängigen Kriechdehnungsanteil zusammensetzen. Der von der Spannungsamplitude abhängige Anteil nimmt dabei mit dem Mittelspannungsniveau zu. Diese These bestätigte sich jedoch nur für Ermüdungsversuche bis zu einem Oberspannungsniveau von etwa $35 \%$ der Betondruckfestigkeit, also einem Beanspruchungsbereich, in dem weitestgehend von einem linear-elastischen Materialverhalten ausgegangen werden kann. Bei höheren Oberspannungen nahmen die plastischen Dehnungen gegenüber den erwarteten Kriechdehnungen überproportional zu. Diese zusätzlichen Dehnungen wurden mit der Rissbildung infolge des zyklischen Beanspruchungsverlaufs in Verbindung gebracht. Das kriechaffine Spannungsniveau des Ermüdungsniveaus liegt demnach zwischen dem Mittelspannungsniveau und dem Oberspannungsniveau. Dabei werden die zeitabhängigen, viskosen Dehnungen von schädigungsinduzierten plastischen Dehnungen infolge des zyklischen Beanspruchungsverlaufs überlagert.

(Bazant \& Kim, 1992) beschreiben die Dehnungen im Ermüdungsversuch auf Grundlage eines bestehenden Kriechmodells. Es wird angenommen, dass die Dehnungszunahme im Ermüdungsversuch und die Kriechkurve auf dem Mittelspannungsniveau des Ermüdungsversuchs die gleiche Form besitzen, die zyklische Beanspruchung jedoch zu einer Beschleunigung des Kriechens führt. Zur Validierung und Kalibrierung des Modells nutzen die Autoren die zuvor aufgeführten Untersuchungen. Ein lastwechselabhängiger, schädigungsinduzierter Dehnungsanteil wird nicht berücksichtigt.

(Holmen, 1979) beschreibt die Verformungen im Ermüdungsversuch über einen zyklen- und einen zeitabhängigen Verformungsanteil. Er schlägt als kriechaffines Spannungsniveau für den zeitabhängigen Verformungsanteil die Summe aus der Mittelspannung und dem quadratischen Mittelwert der Spannungsamplitude vor. Das kriechaffine Spannungsniveau liegt damit entsprechend Gleichung 2.30 zwischen dem Mittelspannungsniveau und dem Oberspannungsniveau und ist von der Belastungsfrequenz unabhängig. 


$$
\begin{aligned}
\sigma_{c r} & =\sigma_{m}+\frac{\sigma_{a}}{\sqrt{2}} \\
& =0,146 \cdot \sigma_{u}+0,854 \cdot \sigma_{o}
\end{aligned}
$$

Die aufgeführten Autoren gehen davon aus, dass während eines Ermüdungsversuchs zeitabhängige Verformungen auftreten. Das kriechaffine Beanspruchungsniveau wird zwischen dem Mittelspannungsniveau und dem Oberspannungsniveau angenommen. Bei Oberspannungen unterhalb der Linearitätsgrenze des Kriechens, scheint das kriechaffine Beanspruchungsniveau dem Mittelspannungsniveau zu entsprechen. Oberspannungen oberhalb der Linearitätsgrenze führen zu einer Erhöhung des kriechaffinen Beanspruchungsniveaus gegenüber dem Mittelspannungsniveau. Die Erhöhung wird durch die Spannungsamplitude definiert. Größere Spannungsamplituden führen zu einer stärkeren Erhöhung. Gleichzeitig wird der Einfluss der Spannungsamplitude durch die Größe der Mittelspannung beeinflusst. Somit ist anzunehmen, dass Phasen des Beanspruchungsverlaufs oberhalb der Linearitätsgrenze zu einer Beschleunigung des Kriechens führen. Diese Beschleunigung könnte im Einklang mit der überproportionalen Dehnungszunahme im Bereich des nichtlinearen Kriechens stehen. Gleichzeitig tritt bei zunehmenden Oberspannungsniveaus ein lastwechselabhängiger, schädigungsinduzierter Dehnungsanteil auf. (Mehmel \& Kern, 1962), die Versuche auch auf hohen Oberspannungsniveaus durchgeführt haben, sowie (Bazant \& Kim, 1992), die diese später auswerteten, trennen die zeitabhängigen und lastwechselabhängigen Anteile nicht voneinander. Sie versuchen die Dehnungen im Ermüdungsversuch allein auf Grundlage von Kriechdehnungen zu beschreiben. Bei (Whaley \& Neville, 1973) und (Rostásy et al., 1975) werden diese Anteile ebenfalls nicht separiert. Der lastwechselabhängige Anteil fällt bei diesen Untersuchungen aber auch weniger ins Gewicht, da die Versuche vorrangig auf niedrigen Spannungsniveaus durchgeführt wurden. Während aber (Whaley \& Neville, 1973) bereits anführen, dass bei höheren Oberspannungsniveaus ein zusätzlicher Anteil aus der Zyklik resultiert, geht (Holmen, 1979) einen Schritt weiter. Er geht davon aus, dass sich die plastischen Dehnungen aus einem lastwechselabhängigen und einem zeitabhängigen Anteil zusammensetzen. Das kriechaffine Beanspruchungsniveau wird entsprechend Gleichung 2.30 veranschlagt aber nicht näher verifiziert. Derzeit gibt es keine genaueren Festlegungen zur Höhe des kriechaffinen Beanspruchungsniveaus in Relation zur Mittelspannung, Spannungsamplitude und der Belastungsfrequenz.

\section{Probekörpererwärmung im Ermüdungsversuch}

Die Erwärmung von ermüdungsbeanspruchten Betonprobekörpern wird durch die Belastungsfrequenz, Spannungsschwingbreite und Versuchsdauer beeinflusst. Größere Spannungsschwingbreiten und höhere Belastungsfrequenzen führen zu einer größeren Wärmefreisetzungsrate im Probekörper und damit zu einer schnelleren Erwärmung (Whaley \& Neville, 1973; Elsmeier \& Lohaus, 2014; von der Haar et al., 2016). 
Die Abbildung 2.26 stellt beispielhaft die Oberflächentemperatur von einem Probekörper im Ermüdungsversuch $\left(S_{o}=0,60 ; S_{u}=0,05 ; f_{p}=10 \mathrm{~Hz}\right)$ dar. Die Bilder wurden mit einer Wärmebildkamera nach 6.000, 22.000 und 42.000 Lastwechseln aufgenommen. Die Bruchlastwechselzahl betrug 43.431 Lastwechsel. Der Probeköper heizte sich während der Versuchsdurchführung deutlich auf. Dabei ist im mittleren Drittel, über die Höhe gesehen, die Oberflächentemperatur nahezu konstant. Diese nimmt in den angrenzenden Bereichen zu den oberen und unteren Rändern deutlich ab. Die Temperaturgradiente ist auf den Wärmeabfluss vom Probekörper in die Druckplatten der Prüfmaschine zurückzuführen. Die Gradiente in horizontaler Richtung basiert auf einem Messfehler. Durch die Krümmung des Probekörpers nimmt der Winkel zwischen Wärmebildkamera und Probekörperoberfläche zum Rand hin zu. Dadurch fällt die Oberflächentemperatur in horizontaler Richtung scheinbar ab. In der Realität ist die Temperatur in Umfangsrichtung konstant.

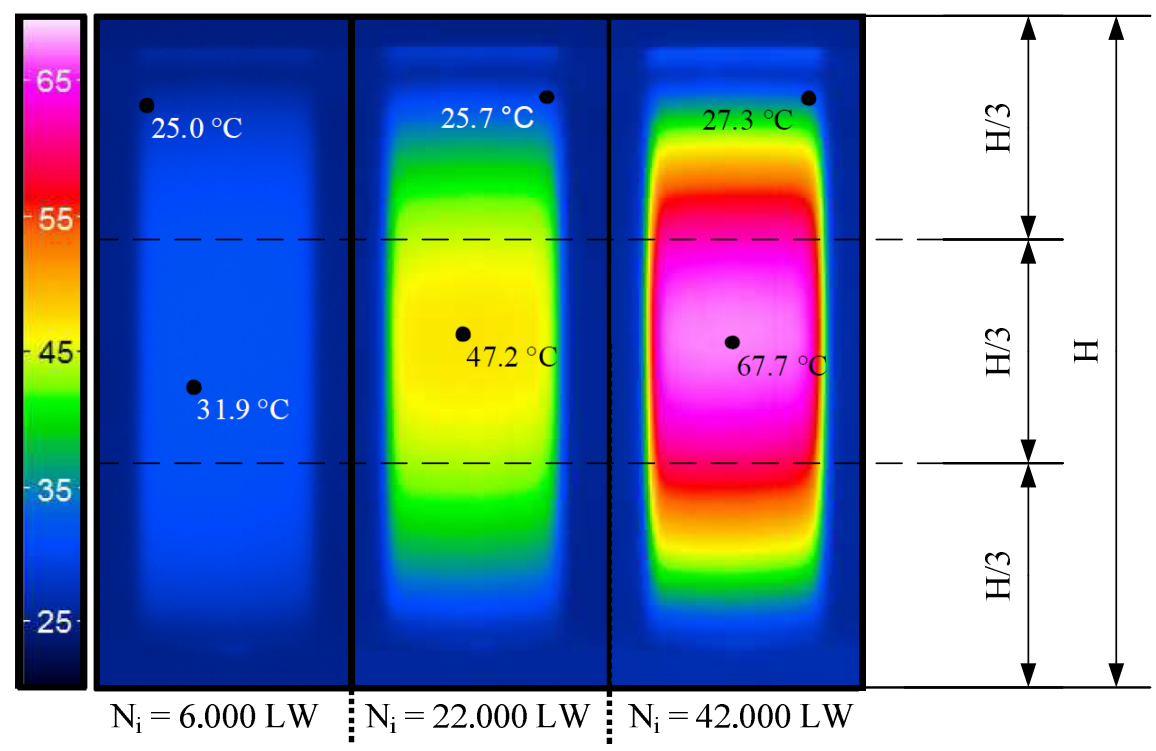

Abbildung 2.26: Temperaturbilder eines ermüdungsbeanspruchten Betonprobekörpers aus (Elsmeier, 2015)

Durch den Wärmeabfluss über die Mantelfläche des Probekörpers an die Umgebungsluft entsteht auch in radialer Richtung des Probekörpers eine Temperaturgradiente. In (Elsmeier, 2015) wurde die Erhöhung der Oberflächentemperatur und der Kerntemperatur auf halber Probenhöhe während der Versuchsdurchführung gemessen und dargestellt. Das Verhältnis ergab sich etwa zu $\Delta T_{\text {Kern }} / \Delta T_{\text {Oberfläche }} \approx 1,3$.

Die Probekörpererwärmung führt zu Temperaturdehnungen. Es handelt sich um eine Ausdehnung des Betonkörpers, die sich mit den Probekörperstauchungen aus der Ermüdungsbeanspruchung überlagern.

\subsection{Energetische Betrachtung der Schädigungsprozesse im Beton}

Bei der energetischen Betrachtungsweise muss das betrachtete System zu seiner Umgebung klar abgegrenzt werden. Es wird nach (Ondracek, 1992) unterschieden zwischen offenen Systemen, bei 
denen das System Materie und Energie mit der Umgebung austauschen kann, geschlossenen Systemen, bei denen nur Energie ausgetauscht werden kann, und isolierten Systemen, bei denen weder Energie- noch Materieaustausch mit der Umgebung stattfindet.

Der Gesamtenergiegehalt eines Systems, wie beispielsweise eines Werkstoffs, besteht aus der mechanischen (potenzielle, kinetische Energie) und der thermochemischen Energie (Bindungsenergie zwischen den Elementarteilchen, thermische Energie). Der absolute Energieinhalt eines Systems ist nicht bekannt, aber die Energieänderung zwischen zwei Systemzuständen kann über Bilanzgleichungen bestimmt werden.

Wird ein Körper mechanisch beansprucht, wird diesem Energie zugeführt. Diese mechanische Energie muss in andere Energieformen gespeichert bzw. umgesetzt werden. Die im Belastungsversuch vom Probekörper aufgenommene Energie lässt sich aus dem Produkt aus aufgebrachter Kraft und dem Verformungsweg in Lastrichtung errechnen (Wischers, 1978a; Wischers 1978b; Siebel, 1989). Ein ideal-elastischer Werkstoff speichert diese Energie in Formänderungsarbeit. Wird dieser Körper bis zur Verformung $\varepsilon_{1}$ belastet, entspricht die gespeicherte Energie der schraffierten Fläche unterhalb der Spannungsdehnungslinie in Abbildung 2.27 (links). Bei Entlastung wird die gesamte gespeicherte Energie wieder zurückgegeben und der Probekörper nimmt seine ursprüngliche Form wieder an.

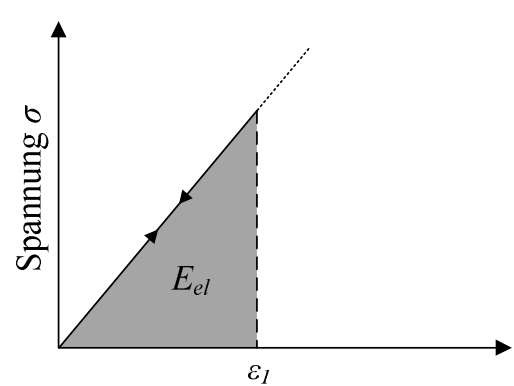

Dehnung $\varepsilon$

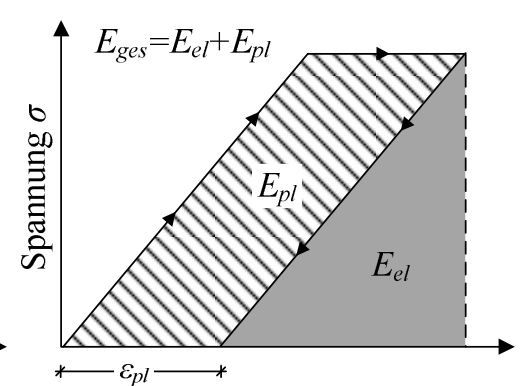

Dehnung $\varepsilon$

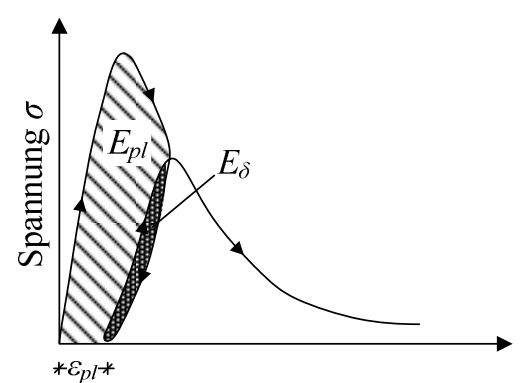

Dehnung $\varepsilon$

Abbildung 2.27: Spannungsdehnungslinie und Energieverteilung eines ideal-elastischen und elasto-plastischen Werkstoffs sowie für Beton nach (Wischers, 1978a)

Ein elasto-plastischer Stoff verhält sich bis zum Erreichen der Fließgrenze zunächst wie ein idealelastischer Werkstoff. Die eingebrachte Energie wird in Form von Verformungsenergie gespeichert. Ab der Fließgrenze wird durch die aufgezwungene Verformung der Prüfmaschine die Kraft nicht weiter gesteigert. Die ab der Fließgrenze konstante Kraft wirkt aber weiterhin in Richtung der Verformung. Die damit einhergehende Energie muss aufgrund des geltenden Energieerhaltungssatzes vom Körper aufgenommen werden. Wird ein elasto-plastischer Werkstoff nach Überschreiten der Fließgrenze wieder entlastet, entspricht die gesamte aufgebrachte Energie der Fläche unterhalb des Belastungspfades der Spannungsdehnungslinie in Abbildung 2.27 (Mitte). Diese Fläche teilt sich auf in den elastischen Anteil, der nach der Entlastung wieder abgegeben wird, und den plastischen Energieanteil, der durch plastische Verformungen $\varepsilon_{p l}$ gespeichert bzw. dissipiert wird. Der elastische Energieanteil entspricht der Fläche unter dem Entlastungspfad. Der plastische Energieanteil $E_{p l}$ ist die Differenz aus der 
Gesamtenergie $E_{\text {ges }}$ und der elastischen Energie $E_{e l}$. Sie wird gespeichert in Form von Rissen als Oberflächenenergie und dem Zermörsern und Umlagern einzelner Werkstoffpartikeln. Geringe Anteile dieser plastischen Energie werden u. a. auch in Wärme umgewandelt bzw. als Schallwellen emittiert.

Bei Beton, der wie in Abbildung 2.27 (rechts) dargestellt ein visko-elastoplastisches Werkstoffverhalten aufweist, setzt sich die aufgebrachte Gesamtenergie nicht nur aus elastischen und plastischen Energieanteilen zusammen, sondern es tritt bei wiederholter Be- und Entlastung noch ein Dämpfungsanteil $E_{\delta}$ auf. Dieser Dämpfungsanteil zeigt sich als Fläche zwischen dem Entlastungspfad und dem folgenden Wiederbelastungspfad (Spooner \& Dougill, 1975; Wischers, 1978a). Dieser Energieanteil wird nach (Teichen, 1968) überwiegend in Wärme umgewandelt.

Die Bruchenergie entspricht nach (Wischers, 1978a) der Energie, die notwendig ist, um einen Betonprüfkörper vollständig zu zerstören und manifestiert sich als Fläche unter der Spannungsdehnungslinie entsprechend Abbildung 2.3. Der durch die Dämpfung aufgezehrte Energieanteil infolge wiederholter Be- und Entlastung bei einer zyklischen Beanspruchung ist nicht Bestandteil dieser Bruchenergie. Diese Annahme spiegelt sich im Envelope-Konzept wider, wonach die Flächen unter der monotonen Spannungsdehnungslinie und unter der Einhüllenden der zyklischen Spannungsdehnungslinie gemäß Abbildung 2.13 identisch sind. (Spooner \& Dougill, 1975) führten hierzu Untersuchungen mittels Schallemissionsmessungen an niederzyklischen, druckbeanspruchten Betonproben durch. Basierend auf diesen Ergebnissen wurde angenommen, dass der in den Hystereseschleifen eingeschlossene Energieanteil nicht schädigungswirksam ist und wie oben genannt mehrheitlich in Wärme umgesetzt wird. Die Versuche von (Karsan \& Jirsa, 1969) und (Maher \& Darwin, 1982) können diese These nicht vollständig untermauern, vgl. Abbildung 2.14. Auf deren Grundlage ist auch in den Hysteresen ein geringer, schädigungswirksamer Energieanteil enthalten.

\subsection{Dehnungsmodelle für ermüdungsbeanspruchten Beton}

Im Folgenden werden ausgewählte Dehnungsmodelle für ermüdungsbeanspruchten Beton dargestellt. Die Modelle beschreiben die Dehnungsentwicklung und Steifigkeitsdegradation unter einer zyklischen Beanspruchung.

\subsubsection{Modelle basierend auf dem Envelope-Konzept}

Das Envelope-Konzept betrachtet die monotone Spannungsdehnungslinie, bzw. eine der monotonen Spannungsdehnungslinie gleichenden Kurve, als Grenzlinie für das Dehnungsverhalten des Betons unabhängig von der Belastungsart und Belastungsreihenfolge. Basierend auf dieser Modellannahme tritt der Ermüdungsbruch bei Erreichen des abfallenden Astes der Spannungsdehnungslinie ein.

Die Steifigkeits- und Dehnungsentwicklung bis zum Erreichen des Bruchzustandes wird in den Ermüdungsmodellen definiert. (Papa, 1993) beschreibt das Materialverhalten ausschließlich auf 
Grundlage einer abnehmenden Probekörpersteifigkeit während der Versuchsdurchführung. Plastische Verformungen wie sie in Abbildung 2.22 dargestellt sind, werden in dem Modell nicht berücksichtigt, vgl. Abbildung 2.28. Dahingegen wird von (Park, 1990) angenommen, dass sich die Mittellinien der Hystereseschleifen alle in einem Punkt treffen. Der Schnittpunkt wird bei $\left(-0,5 \varepsilon_{c} \mid-f_{c}\right)$ angenommen, vgl. Abbildung 2.29. Die Lage des Schnittpunktes wird von Park nicht weiter diskutiert. In dem Modell wird die Schädigungsentwicklung über die Zunahme plastischer Dehnungen repräsentiert. Die plastische Dehnungszunahme pro Lastwechsel wurde mittels Regressionsanalysen anhand von Versuchsdaten bestimmt.

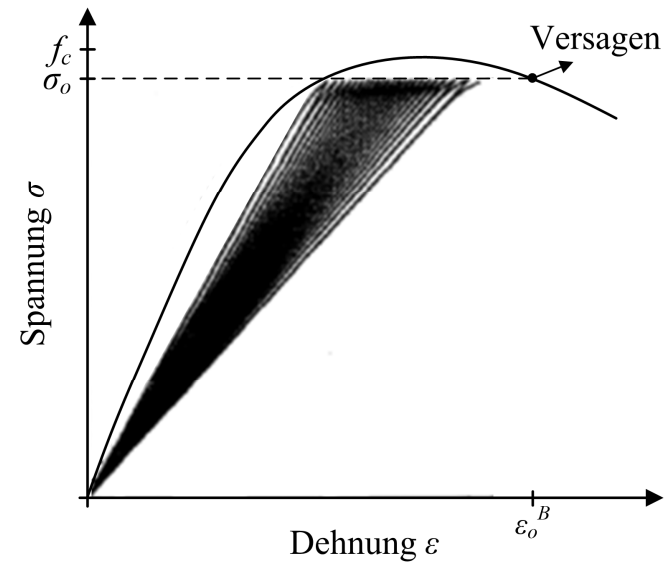

Abbildung 2.28: Modellvorstellung aus (Papa, 1993)

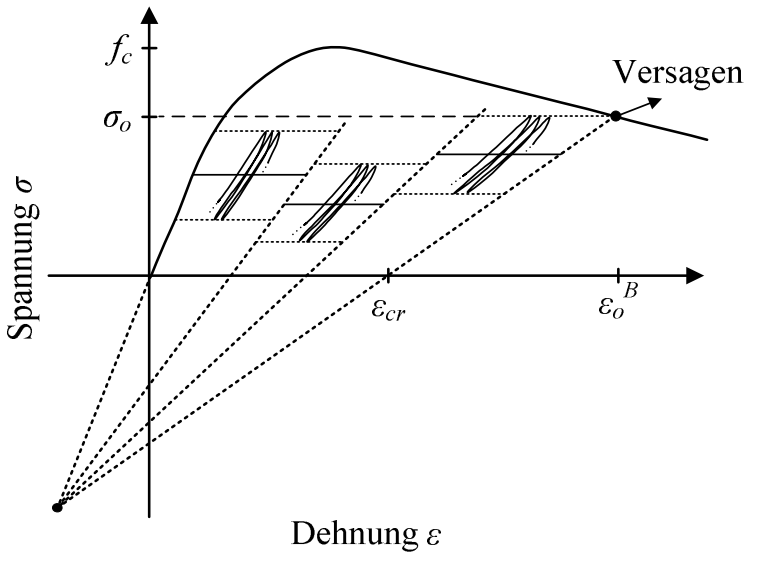

Abbildung 2.29: Modellvorstellung aus (Park, 1990)

Viskose Dehnungen werden in den angeführten Modellen nicht näher betrachtet. Bei der plastischen Dehnungszunahme in dem Modell von Park wird aber auch nicht zwischen schädigungsinduzierten und viskosen Verformungen weiter differenziert. Die experimentell bestimmte Verformungszunahme pro Lastwechsel enthält theoretisch beide Anteile. Die Modelle stehen u. a. im Widerspruch zu den Versuchsergebnissen von (Awad \& Hilsdorf, 1971), die Ermüdungsbruchdehnungen außerhalb der Envelope-Kurve feststellten, vgl. Abbildung 2.23.

\subsubsection{Energetisches Ermüdungsschädigungsmodell}

Basierend auf den Beobachtungen in (Spooner \& Dougill, 1975) und (Spooner et al., 1976), in denen mechanische Schädigung als energetischer dissipativer Prozess betrachtet wird, wird von (Pfanner, 2003) ein energetisches Ermüdungsschädigungsmodell vorgeschlagen. Der Ansatz beruht auf der Hypothese, dass die während eines Ermüdungsprozesses vom Beton dissipierte Energie genau der Energie entspricht, die unter monotoner Beanspruchung aufgewendet werden muss, um den gleichen Schädigungszustand herzustellen.

Die dem energetischen Ermüdungsschädigungsmodell zugrunde gelegte monotone Spannungsdehnungslinie entstammt dem Materialmodell von (Pölling, 2000). Dieser formulierte das Betonversagen auf Grundlage einer spannungsbasierten elasto-plastischen Kontinuumsschädigungstheorie. Die zugehörige Spannungsdehnungslinie für den monoton beanspruchten Beton wird in Abbildung 2.30 dargestellt. In dem ersten Bereich der Spannungsdehnungslinie bis zu einer Spannung von einem Drittel der Bruchfestigkeit wird ein linear elastisches 
Materialverhalten angenommen. Es treten weder plastisches Fließen noch Materialschädigungen auf. Im zweiten Bereich, dem sogenannten Vorbruchbereich, steigt die Spannung bis auf die Bruchspannung $f_{c}$ an. Zur Beschreibung des Verlaufes wird die Formulierung nach Model Code 1990 herangezogen. Im dritten Bereich erfolgt die Lokalisierung der Schädigung. Zuvor war die dissipierte Energie $g_{c u}$ diffus über den Probekörper verteilt. In dem sich einstellenden Rissband kommt es zu einer Materialentfestigung, die zu einer Entlastung in den übrigen Bereichen führt. Der Verlauf der Spannungsdehnungslinie wird im Wesentlichen durch die Bruchenergie $G_{c l}$ (Summe aus $g_{c l l}$ und $g_{c l 2}$ ), die als Materialparameter aufzufassen ist, bestimmt.
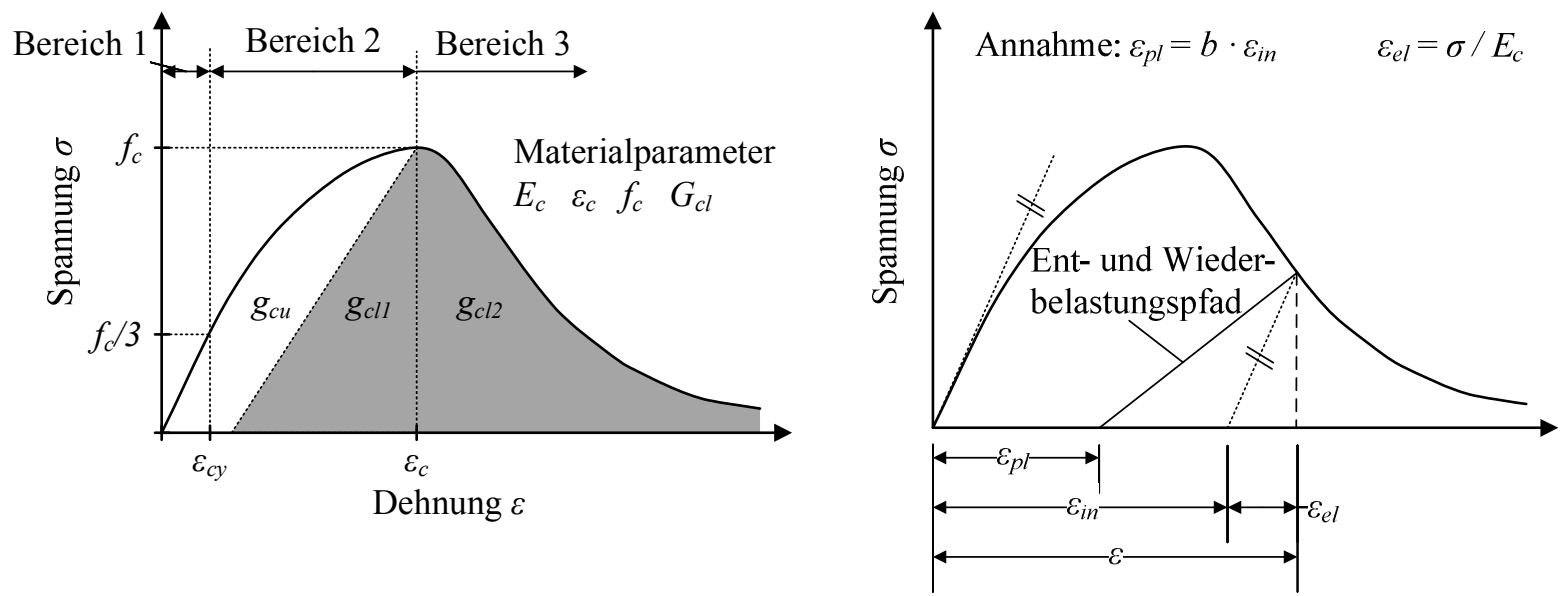

Abbildung 2.30: Spannungsdehnungslinie von (Pölling, 2000) nach (Pfanner, 2003)

Nach Pfanner entspricht die im Ermüdungsprozess dissipierte Energie der Fläche unterhalb der umhüllenden Spannungsdehnungslinie des Ermüdungsversuchs. Somit wird nur die innere Energieänderung betrachtet, die makroskopisch zu einem Dehnungszuwachs führt. Durch Gleichsetzen der Energien unter monotoner und zyklischer Beanspruchung gelingt Pfanner die Formulierung des Versagenszustandes sowie der Materialdegradation während des Ermüdungsversuchs auf Grundlage der monotonen Spannungsdehnungslinie des Betons. Grundsätzlich treten gemäß dem energetischen Modell Versagensdehnungen auf, die außerhalb der monotonen Spannungsdehnungslinie liegen. Wie in Abbildung 2.31 leicht zu erkennen ist, sind diese entscheidend vom Verlauf der Spannungsdehnungslinie im Nachbruchbereich abhängig.

Das Modell wurde in (Göhlmann, 2009) um die Betrachtungsmöglichkeit mehrstufiger Ermüdungsbeanspruchungen erweitert und in einer kommerziellen Finite-Elemente Software eingebunden.

Die genannten Autoren, Pfanner und Göhlmann, verifizierten das energetische Modell bzw. dessen Erweiterung an Ermüdungsversuchen aus der Literatur bzw. an wenigen durchgeführten Einzelversuchen. Die experimentellen Dehnungsverläufe und Steifigkeitsdegradationen konnten mit dem Modell nachgerechnet werden. Es ist jedoch zu beachten, dass die für die Nachrechnung erforderlichen abfallenden Äste der monotonen Spannungsdehnungslinie, die ausschlaggebend für die Ergebnisse des Ermüdungsmodells sind, nur abgeschätzt wurden, da diese in der Literatur 
nicht vorlagen bzw. im Rahmen der Versuchsdurchführungen nicht ermittelt wurden. Pfanner überprüfte das Modell u. a. an den Versuchsergebnissen von (Holmen, 1979). Er wählte die Bruchenergie so, dass die Nachbruchkurve sehr steil abfiel und die Ermüdungsbruchdehnungen aus Abbildung 2.24 rechtsseitig des abfallenden Astes lagen.

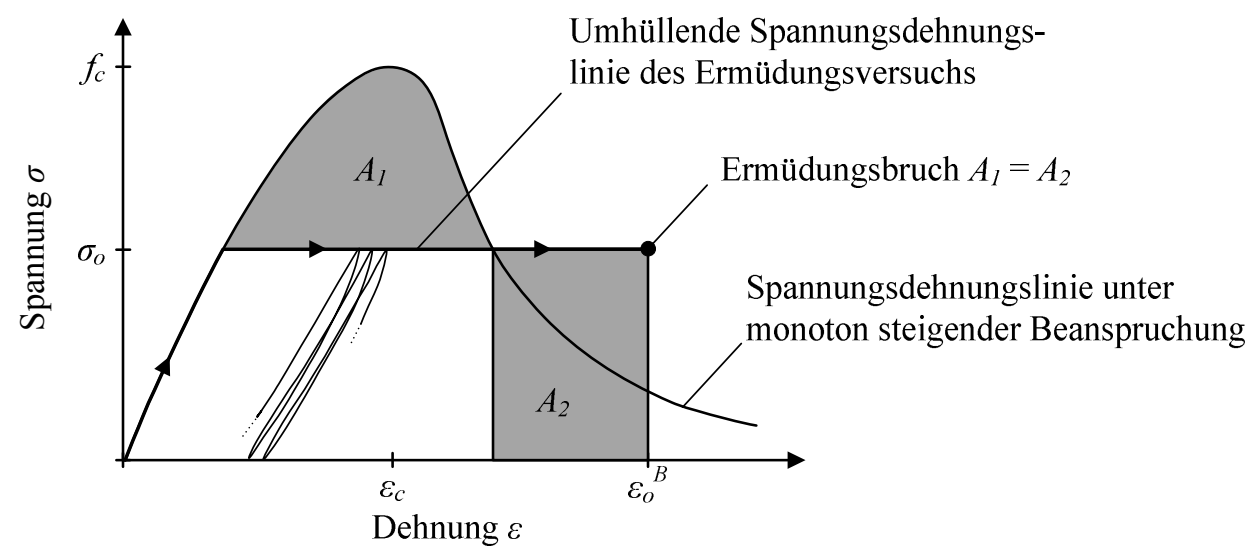

Abbildung 2.31: Definition des Ermüdungsbruchs nach (Pfanner, 2003) 


\section{Hypothese und Vorgehen}

\subsection{Einordnung der Arbeit in den Kontext der Literaturstudie}

In der Vergangenheit fanden umfangreiche Untersuchungen $\mathrm{zu}$ den Bruchlastwechselzahlen ermüdungsbeanspruchter Betonproben statt. In den Untersuchungen wurde u. a. der Einfluss unterschiedlicher Betonzusammensetzungen, Umgebungs-, Prüf- und Lagerbedingungen erforscht. Die Ergebnisse wurden teils in Form von Wöhlerlinien zusammengefasst und seitens der Normung zur Bemessung ermüdungsbeanspruchter Betonbauwerke zur Verfügung gestellt. Die Bruchlastwechselzahlen sind zwar starken Streuungen unterworfen, aber gleichzeitig zeigen Ermüdungsuntersuchungen an Stahlbetonbauteilen, dass das Ermüdungsversagen des Betons i. d. R. nicht vor dem Ermüdungsversagen des Betonstahls auftritt (Kuryllo et al., 1976; Schläfli, 1999). Im Beton finden somit Mechanismen bzw. Prozesse statt, die seitens der Wöhlerlinien nicht abgebildet werden, aber die Lebensdauer des Betons auf Bauteilebene günstig beeinflussen. Mit dem Ziel, diese Mechanismen bzw. Prozesse besser verstehen zu können, wurde in den letzten Jahren zunehmend der Blick auf die Steifigkeitsdegradation und auf das Verformungsverhalten des Betons im Ermüdungsversuch gerichtet (Pfanner, 2003; Ibuk, 2008; Göhlmann, 2009; Oneschkow, 2014).

In Bezug auf das Verformungsverhalten spielt die Ermüdungsbruchdehnung eine maßgebende Rolle. In der Literatur sind diverse Überlegungen dokumentiert, um die Ermüdungsbruchdehnung als Versagenskriterium zu charakterisieren. Die Ermüdungsbruchdehnungen werden in diesem Zusammenhang mit dem Verformungsverhalten unter monoton steigender Beanspruchung ins Verhältnis gesetzt. Bestünde ein derartiger Zusammenhang, könnte das Verformungsverhalten eines ermüdungsbeanspruchten Betons mithilfe weniger kurzzeitiger Druckversuche beurteilt werden, ohne dass zeit- und kostenintensive Ermüdungsversuche notwendig wären. In der Literatur sind hierzu drei Hauptansätze beschrieben, die wie folgt benannt werden können (vgl. auch Abschnitt 2.4.2 und 2.6):

- Die Ermüdungsbruchdehnung entspricht der Bruchdehnung unter monoton steigender Beanspruchung.

- Die Ermüdungsbruchdehnung liegt auf dem abfallenden Ast der Envelope-Kurve.

- Die Ermüdungsbruchdehnung übertrifft den abfallenden Ast der Envelope-Kurve.

Für alle drei Annahmen liegen in der Literatur Versuchsergebnisse vor, die diese belegen bzw. widerlegen. Dabei müsste aber grundsätzlich die Frage gestellt werden, wie gut die Annahmen sein können, wenn die Bruchdehnung unter monoton steigender Beanspruchung als auch die Envelope-Kurve wesentlich von der Belastungsgeschwindigkeit bzw. der Versuchsdauer abhängen. 
Des Weiteren zeigt die Literaturstudie, dass die Ermüdungsbruchdehnungen von zeitabhängigen, viskosen Verformungen beeinflusst werden. Verschiedene Forscher versuchten anhand experimenteller Untersuchungen die viskosen Verformungen mit den Kriechverformungen unter einer dauerhaften Druckbeanspruchungen ins Verhältnis zu setzen. Demnach liegt das kriechaffine Beanspruchungsniveau zwischen dem Mittelspannungsniveau und dem Oberspannungsniveau des zyklischen Beanspruchungsverlaufs. Eine genauere Quantifizierung dessen ist aber bislang nicht gelungen.

Im Weiteren zeigt die Literaturauswertung, dass während eines Ermüdungsversuchs merkliche Probekörpererwärmungen auftreten. Die Probekörpererwärmung wird vom Beanspruchungsniveau, der Belastungsfrequenz sowie der Prüfdauer beeinflusst und ruft Temperaturdehnungen hervor, die sich mit den Verformungen aus der Druckschwellbeanspruchung überlagern.

Die auftretenden thermischen und viskosen Dehnungen haben zur Folge, dass die Ermüdungsbruchdehnungen nicht mehr auf einen festen Wert bzw. auf einen definierten Kurvenverlauf, der unter monoton steigenden Beanspruchung auftritt, bezogen werden können, sondern von weiteren Faktoren wie der Spannungsschwingbreite, der Belastungsfrequenz und der Versuchsdauer beeinflusst werden. Es muss angenommen werden, dass sich die Gesamtdehnungen aus unterschiedlichen Dehnungsanteilen zusammensetzen, die sich in Abhängigkeit der zyklischen Beanspruchungscharakteristik individuell entwickeln. So ist davon auszugehen, dass bei hohen Belastungsfrequenzen die Temperaturverformungen stärker auftreten als bei geringeren Frequenzen. Andererseits führen geringere Frequenzen zu höheren Prüfdauern und damit zu größeren viskosen Dehnungen als höhere Frequenzen.

\subsection{Hypothese}

Es wird von der Hypothese ausgegangen, dass im Ermüdungsversuch aus der zyklischen Beanspruchung vier Dehnungsanteile resultieren. Bei diesen Anteilen handelt es sich um einen elastischen Dehnungsanteil $\varepsilon_{e l}$, einem lastwechselabhängigen, schädigungsinduzierten Dehnungsanteil $\varepsilon_{d}$, einem zeitabhängigen, viskosen Dehnungsanteil $\varepsilon_{c r}$ und um einen Temperaturdehnungsanteil $\varepsilon_{t}$. Die Gesamtverformungen im Ermüdungsversuch $\varepsilon_{f a t}$ ergeben sich entsprechend Gleichung 3.1 aus der Summe der einzelnen Verformungsanteile. Die Dehnungsanteile werden von der Charakteristik des Beanspruchungsverlaufs und den Materialeigenschaften individuell beeinflusst. Es handelt sich damit um ein additives Dehnungsmodell; die einzelnen Dehnungsanteile entwickeln sich unabhängig voneinander und ergeben gemeinsam die messbare Gesamtverformung. Im Folgenden werden ausschließlich Verformungen in Belastungsrichtung betrachtet.

$$
\varepsilon_{f a t}=\varepsilon_{e l}+\varepsilon_{d}+\varepsilon_{c r}+\varepsilon_{t}
$$

Da Ermüdungsversuche in der Regel nur wenige Stunden bis Tage dauern werden auftretende Schwindverformungen des Betons in Gleichung 3.1 nicht weiter berücksichtigt. Grundsätzlich könnten diese aber über einen weiteren Summanden erfasst werden. 
Der elastische Anteil beschreibt die Dehnungsdifferenz zwischen dem Oberspannungsniveau $S_{o}$ und dem Entlastungsniveau $(S=0)$. Die gekrümmte Form der Be- und Entlastungsäste wird im Rahmen dieser Modellvorstellung vernachlässigt. Sie ist für die Betrachtung der Gesamtverformungen nicht von Interesse. Die Dehnungsdifferenz wird über den Sekantenmodul der Entlastungsäste beschrieben, vgl. Gleichung 3.2 und Abbildung 3.1 (links).

$$
\varepsilon_{e l}=\frac{\sigma_{o}}{E_{s}}
$$

Der Sekantenmodul nimmt analog zu Abbildung 2.21 mit zunehmender Lastwechselzahl ab, dementsprechend vergrößert sich der elastische Dehnungsanteil. Die inkrementelle Steifigkeitsänderung wird durch das Oberspannungsniveau, aber auch durch die Betonfestigkeitsklasse beeinflusst. Wie in Abschnitt 2.4.2 erläutert, hängt die Steifigkeitsdegradation einerseits mit der Verdichtung des Zementsteins, andererseits mit der Rissbildung parallel und vertikal zur Lastrichtung zusammen. Höherfeste Betone sind infolge ihrer geringeren Porosität durch eine geringere Viskosität gekennzeichnet. Hierdurch ist eine geringere Verdichtung des Zementsteins während der Versuchsdurchführung zu erwarten. Dementsprechend nimmt die Neigung zur Rissbildung senkrecht zur Lastrichtung ab. Zudem führt der verbesserte Verbund zwischen Gesteinskörnern und Zementleim hochfester Betone zu einer geringeren Rissbildung parallel zur Lastrichtung. Dadurch ist die Steifigkeitsdegradation bis zum Bruch weniger ausgeprägt als bei normalfesten Betonen. Im elastischen Dehnungsanteil sind allerdings nur die Auswirkungen der Rissbildung bzw. der Verdichtung auf das elastische Verformungsverhalten des Betons enthalten. Die aus den Rissbildungs- und Verdichtungsprozessen selbst entstehenden Verformungen werden über die schädigungsinduzierten und viskosen Verformungsanteile berücksichtigt. Diese Darstellungsmethode entspricht dem Vorgehen zur Beschreibung der Verformungen im Kriechversuch. Bei diesem werden ebenfalls die schädigungsinduzierten und viskosen Verformungsanteile, die während des Aufbringens der Dauerbeanspruchung auftreten, nicht den elastischen Verformungen, sondern dem Kriechen zugeschrieben.
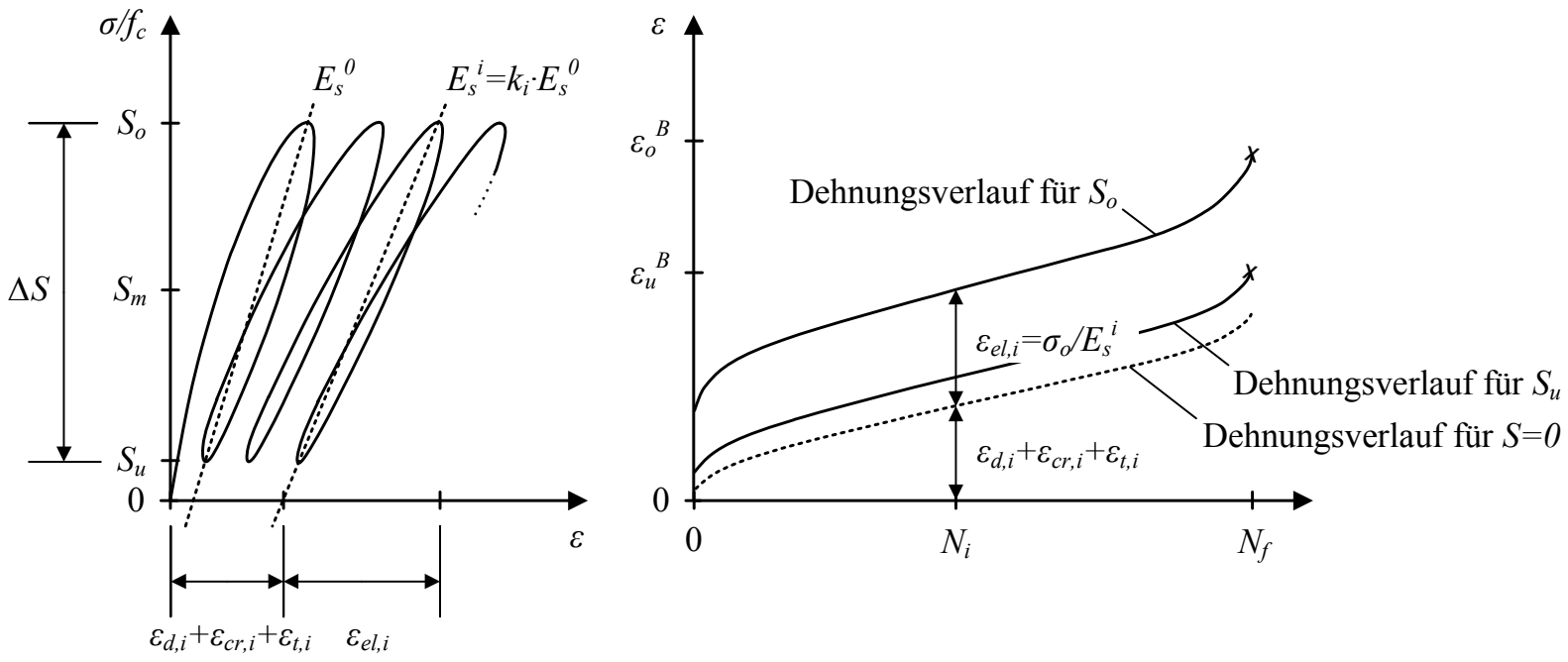

Abbildung 3.1: Modellvorstellung zum elastischen Dehnungsanteil 
Ermüdungsversuche werden üblicherweise auf einem Unterspannungsniveau von ungleich null durchgeführt. Oft wird das Unterspannungsniveau zu $5 \%$ der Betondruckfestigkeit gewählt. Die Ursache ist regelungstechnisch begründet, um einen Kontaktverlust zwischen Prüfmaschine und Probekörper während der Versuchsdurchführung zu vermeiden. Daher ist die Dehnungsdifferenz zwischen Oberspannungsniveau und Entlastungsniveau im Ermüdungsversuch messtechnisch nicht erfassbar. Sie muss auf Grundlage des Sekantenmoduls, bestimmt aus der Dehnungsdifferenz zwischen Oberspannungsniveau und Unterspannungsniveau, extrapoliert werden, vgl. Abbildung 3.1 (links). Durch die gekrümmte Form des Entlastungsastes resultiert hieraus ein Fehler. Dieser wird in Anbetracht der Gesamtverformungen als unerheblich erachtet. Es soll versucht werden, diesen im Zuge der geplanten Versuchsdatenauswertung zu quantifizieren.

Die Temperaturdehnungen resultieren aus der Probekörpererwärmung während des Ermüdungsversuchs. Die Erwärmung steht im Zusammenhang mit der Umwandlung von Dämpfungsenergie in Wärmeenergie, vgl. Abschnitt 2.5. Die Dämpfungsenergie entspricht der eingeschlossenen Fläche zwischen den Ent- und Wiederbelastungspfaden des zyklischen Beanspruchungsverlaufs im Spannungsdehnungsdiagramm. Damit ist die freiwerdende Wärmemenge pro Zeiteinheit von der Größe der Hysteresefläche und der Zahl der Lastwechsel pro Zeiteinheit abhängig. Die Größe der Hysteresefläche wird durch die Spannungsschwingbreite beeinflusst. Die Zahl der Lastwechsel pro Zeiteinheit wird von der Belastungsfrequenz bestimmt. Der Wärmeabfluss über die Betonoberflächen an die Umgebung ist von der Wärmeleitfähigkeit, den Konvektionsbedingungen und der bezogenen Oberfläche des Probekörpers abhängig. Im Betonkörper treten in longitudinaler und radialer Richtung Temperaturgradienten auf, die zu Eigenspannungen und Dehnungen führen. Die mittlere Temperaturänderung der Probekörper ist auf Grundlage von Temperaturmessungen während der Versuchsdurchführung abzuschätzen, um die auftretenden Temperaturdehnungen zu berechnen. Diese ergeben sich entsprechend Gleichung 3.3 aus der mittleren Temperaturänderung $\Delta T$ multipliziert mit dem Wärmeausdehnungskoeffizienten des Betons $\alpha_{T}$.

$$
\varepsilon_{t}=\alpha_{T} \cdot \Delta T
$$

Der elastische und thermische Dehnungsanteil ist auf Grundlage von Verformungs- und Temperaturmessungen im Ermüdungsversuch und anschließenden Nachlaufberechnungen verhältnismäßig einfach abzuschätzen. Demgegenüber ist zwischen den schädigungsinduzierten und viskosen Verformungen schwieriger $\mathrm{zu}$ differenzieren. Die schädigungsinduzierten Verformungen resultieren aus der inkrementellen Schädigungszunahme je Lastwechsel. Sie sind die Folge einer zunehmenden Materialzerrüttung, Rissbildung und der sukzessiven Verschiebung von Rissflanken. Die viskosen Verformungen setzen sich aus irreversiblen und visko-elastischen Verformungen zusammen und resultieren aus der andauernden Wirkung der zyklischen Beanspruchung. Da die visko-elastischen Verformungen reversibel sind, gibt es hier im Grundsatz keine Überschneidungen mit den schädigungsinduzierten Dehnungen. Allerdings wird der viskoelastische Anteil nur nach einer ausreichend lange andauernden Entlastungsphase sichtbar. Die viskosen, irreversiblen (Fließ-)Verformungen unter Dauerlasten oberhalb der Linearitätsgrenze 
sind auch mit Rissbildungsprozessen verknüpft, sodass hier eine Überschneidung mit den schädigungsinduzierten Verformungen besteht. Insgesamt stellen sich somit alle drei Anteile im Ermüdungsversuch als plastische Verformungszunahme dar und eine eindeutige Differenzierung zwischen schädigungsinduzierten und viskosen Verformungsanteilen ist nicht mehr möglich. Die Trennung dieser Anteile ist nur definitionsgemäß bzw. modellmäßig zu bewerkstelligen.

Es wird angenommen, dass aus dem zyklischen Beanspruchungsverlauf viskose Dehnungen resultieren, die aus dem Kriechverhalten des Betons unter dauerhaften Druckbeanspruchungen superponiert werden können, vgl. beispielsweise Abbildung 2.12. Es folgt ein Verformungsverlauf, der als viskoser Dehnungsanteil bezeichnet wird. Es handelt sich um einen zeitabhängigen Dehnungsanteil, der den Annahmen und Modellvorstellungen zum Betonkriechen unter einer dauerhaften Druckbeanspruchung entspricht. Er enthält visko-elastische und viskoplastische Dehnungsanteile. Das kriechaffine Beanspruchungsniveau wird entsprechend der Literaturstudie vom Mittelspannungsniveau des zyklischen Beanspruchungsverlaufs dominiert und von der Spannungsamplitude und unter Umständen von der Belastungsfrequenz beeinflusst. Das kriechaffine Beanspruchungsniveau wird nachfolgend genauer quantifiziert. Der Verformungsverlauf ist in erster Linie zeitabhängig zu formulieren. Er ist über die Beziehung $N=f_{p} \cdot t$ mit der Lastwechselzahl verknüpft.

Der schädigungsinduzierte Verformungsanteil resultiert aus der Zyklik des Beanspruchungsverlaufs als Folge der Beanspruchungsänderung pro Lastwechsel. Diese modellmäßige Betrachtungsweise weicht damit von der üblichen Betrachtungsweise im Bereich der Kriechforschung ab. Im Bereich der Kriechforschung werden die schädigungsinduzierten und viskosen Verformungsanteile vollständig dem Betonkriechen zugeordnet. Diese Betrachtungsweise stellt sich aber im Falle von Ermüdungsuntersuchungen als unzureichend dar. Geht man von Ermüdungsversuchen aus, die auf gleichen Beanspruchungsniveaus aber unterschiedlichen Belastungsfrequenzen durchgeführt werden, so ist anzunehmen, dass beide Versuche nach der gleichen lastwechselabhängigen, schädigungsinduzierten Dehnungszunahme bzw. Lastwechselzahl versagen. Durch die unterschiedlichen Belastungsfrequenzen bzw. Versuchsdauern treten aber unterschiedlich große viskose Verformungen auf. Würden die Verformungsanteile gemeinsam betrachtet werden, würde der lastwechselabhängige, schädigungsinduzierte Dehnungsanteil unbekannt bleiben. Dabei bestünde aber die Möglichkeit, dass gerade dieser Dehnungsanteil für die Formulierung eines Versagenskriteriums von Bedeutung ist, da er indirekt die Gefügeschädigung repräsentiert. Zudem wäre die Summe aus beiden Anteilen schwierig zu interpretieren. Sie würde sich lastwechsel- als auch zeitabhängig entwickeln.

Es wird angenommen, dass sich die einzelnen Verformungsanteile des additiven Dehnungsmodells unabhängig voneinander entwickeln und nicht gegenseitig beeinflussen. Das bedeutet, dass die Steifigkeitsdegradation des Betons nicht $\mathrm{zu}$ einer Beschleunigung des Kriechens führt. Gleichzeitig wird der Einfluss der Probekörpertemperatur auf die Steifigkeit, Festigkeit und die Kriechfähigkeit des Betons nicht berücksichtigt. Die Interaktion dieser 
Einflüsse stellt im Sinne der hier dargestellten Modellvorstellung eine Erweiterung bzw. Weiterentwicklung dar.

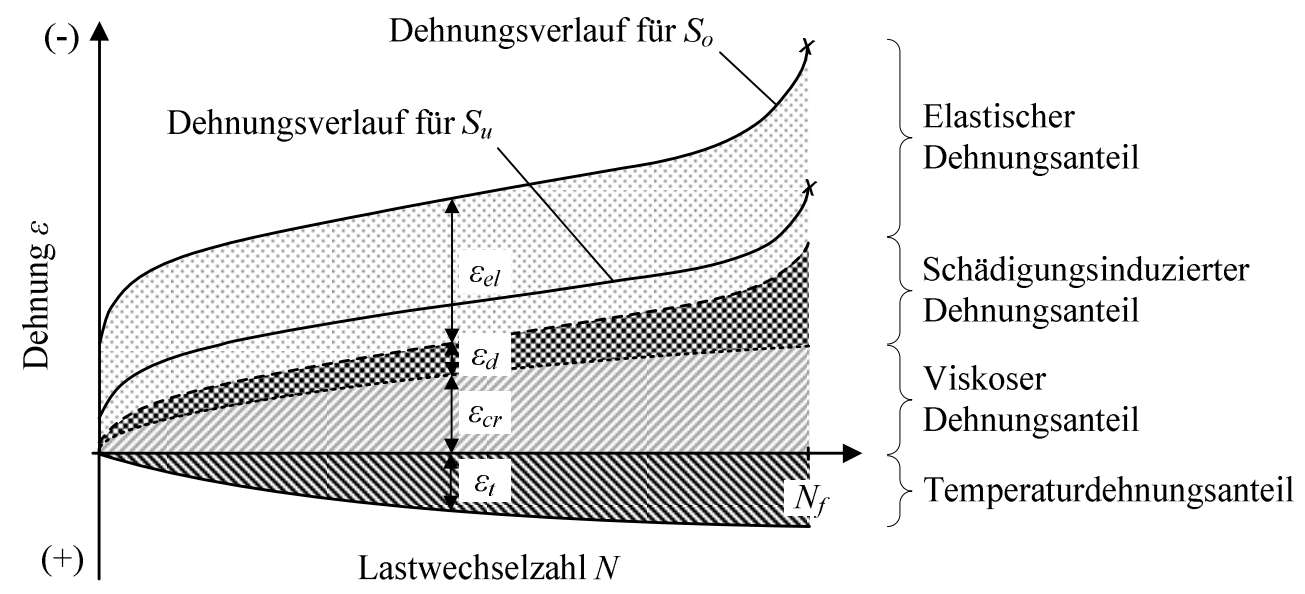

Abbildung 3.2: Dehnungsanteile im Ermüdungsversuch

Die Abbildung 3.2 stellt die beschriebenen Dehnungsanteile über der Lastwechselzahl qualitativ dar. Bei den elastischen, schädigungsinduzierten und viskosen Dehnungen im druckschwellbeanspruchten Ermüdungsversuch handelt es sich um Probekörperstauchungen, die Dehnungswerte sind negativ. Die Probekörpererwärmung führt zu Probekörperausdehnungen, die Dehnungswerte sind dementsprechend positiv definiert. Durch das unterschiedliche Vorzeichen der Dehnungsanteile ergibt sich die experimentell messbare Probekörperverformung im Ermüdungsversuch entsprechend Gleichung 3.4 und Abbildung 3.3.

$$
\left|\varepsilon_{f a t}\right|=\left|\varepsilon_{e l}\right|+\left|\varepsilon_{d}\right|+\left|\varepsilon_{c r}\right|-\left|\varepsilon_{t}\right|
$$

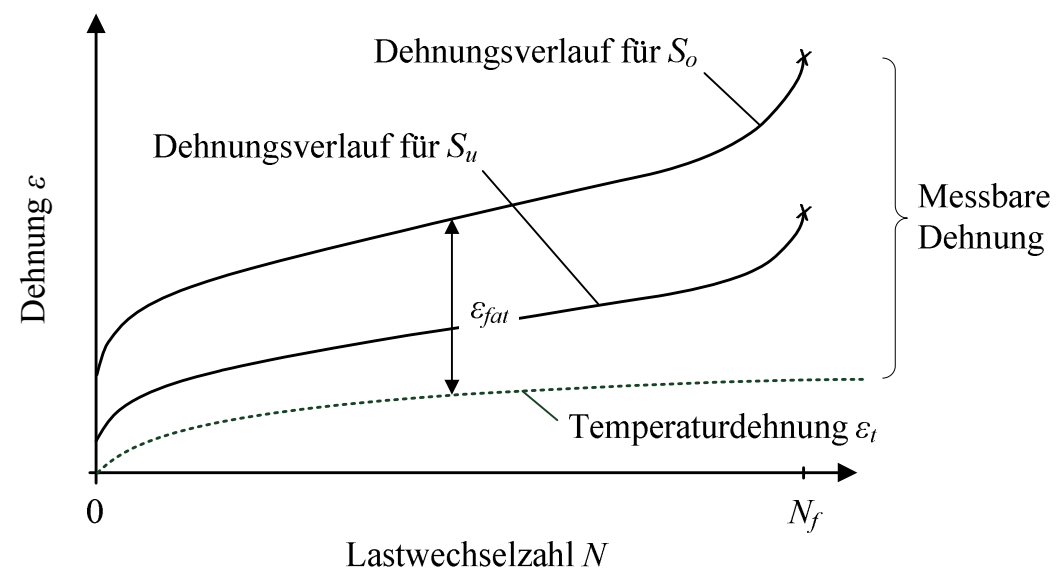

Abbildung 3.3: Messbare Probekörperdehnung im Ermüdungsversuch

\subsection{Ziel und Vorgehen}

Das Hauptziel der Arbeit ist die Bestimmung des kriechaffinen Beanspruchungsniveaus bei ermüdungsbeanspruchten Betonkörpern. Damit soll ermöglicht werden, den viskosen Dehnungsanteil von Betonkörpern im Ermüdungsversuch abzuschätzen. Auf Grundlage des 
kriechaffinen Beanspruchungsniveaus und den bekannten Zusammenhängen für die elastischen und thermischen Dehnungen (vgl. Gleichungen 3.2 und 3.3) können folglich drei der vier Dehnungsanteile des additiven Dehnungsmodells beschrieben werden, wodurch auch der schädigungsinduzierte Dehnungsanteil identifizierbar wird. Die Trennung der einzelnen Dehnungsanteile bietet die Möglichkeit, ein tieferes Verständnis für die stattfindenden Verformungsprozesse zu erlangen, sowie langfristig gesehen ein Versagenskriterium auf dieser Basis zu formulieren.

\subsubsection{Modellentwicklung}

Ausgangspunkt für die Bestimmung des kriechaffinen Beanspruchungsniveaus ist die Annahme, dass der aus dem zyklischen Beanspruchungsverlauf resultierende viskose Verformungsverlauf, aus dem Kriechverhalten des Betons unter dauerhaften Druckbeanspruchungen superponiert werden kann. Damit wird das Superpositionsprinzip der Kriechdehnungen (s. Abschnitt 2.2.4) auf den viskosen Dehnungsanteil von ermüdungsbeanspruchten Beton übertragen. Das kriecherzeugende Beanspruchungsniveau kann damit auf Grundlage einer im Bereich der Kriechforschung etablierten Modellvorstellung abgeleitet werden. Auf dieser Basis können formale Zusammenhänge zwischen dem kriechaffinen Beanspruchungsniveau und dem Mittelspannungsniveau, der Spannungsamplitude sowie der Belastungsfrequenz im Ermüdungsversuch aufgedeckt und analysiert werden. Im Anschluss daran werden die Ergebnisse experimentell plausibilisiert und verifiziert.

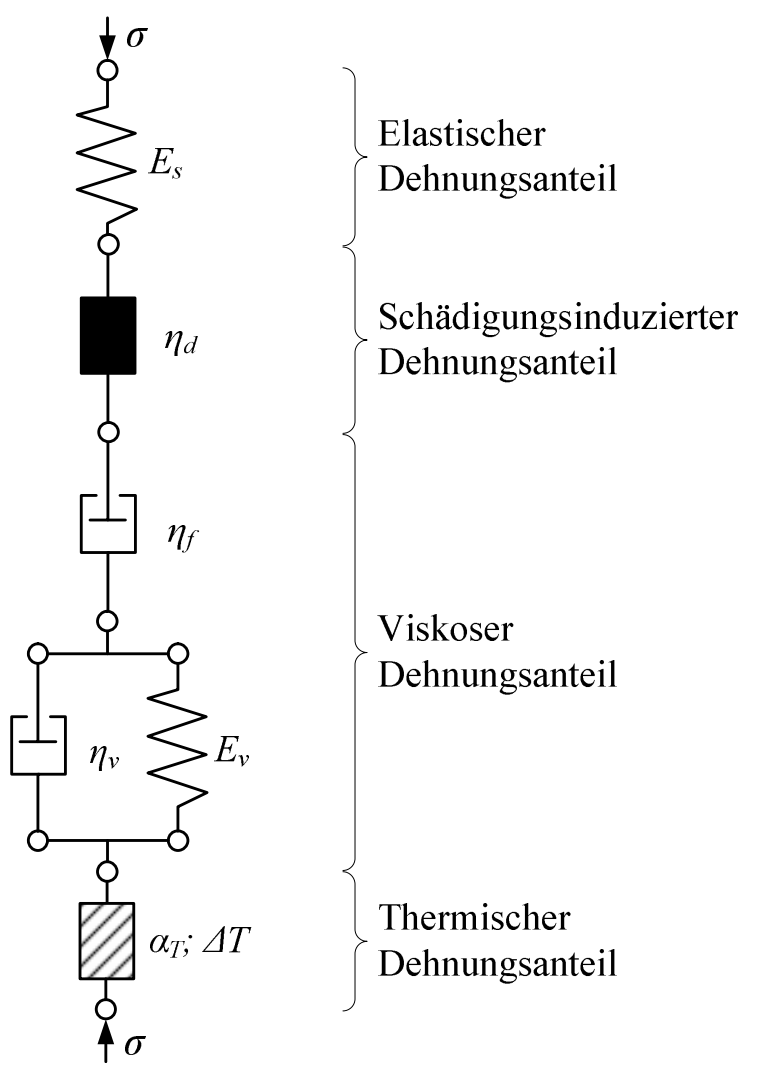

Abbildung 3.4: Rheologische Modellvorstellung 
Zur Veranschaulichung der einzelnen Dehnungsanteile in dem additiven Dehnungsmodell und zur Bestimmung des kriechaffinen Beanspruchungsniveaus wird eine rheologische Modellvorstellung gewählt, vgl. Abbildung 3.4. In dem Modell werden die elastischen Verformungen über ein Federelement abgebildet. Die Steifigkeit des Federelements ist in Abhängigkeit der Lastwechselzahl degradierend zu definieren. Es bildet die Entwicklung der Sekantensteifigkeit $E_{s}$ des Betonkörpers im Ermüdungsversuch ab. Die schädigungsinduzierten Verformungen werden über ein Element des Typs Black Box symbolisiert. Die Verformungen wachsen mit zunehmender Lastwechselzahl an, deren Entwicklung ist derzeit jedoch nicht näher bekannt und soll im Rahmen dieser Arbeit erstmals bestimmt werden. Die genaue Beschreibung auf Basis rheologischer Grundelemente wird dementsprechend nicht weiter behandelt und bietet Raum für zukünftige Arbeiten. Die viskosen Verformungen werden in Anlehnung an ein Burgers-Modell über einen Kelvin-Körper, zur Abbildung visko-elastischer Verformungen, und einem Dämpfer, zur Abbildung visko-plastischer Verformungen, abgebildet. Die thermischen Verformungen werden mithilfe eines Dehnkörpers beschrieben. Die Elongation des Probekörpers ergibt sich aus der mittleren Temperaturänderung $\Delta T$ und dem Wärmeausdehnungskoeffizienten $\alpha_{T}$ des Betons.

Die viskosen Verformungskomponenten des Modells $\left(\eta_{f}, \eta_{v}, E_{v}\right)$ sollen zunächst an das Kriechverhalten eines Referenzbetons angepasst werden. Anschließend werden zyklische Beanspruchungsverläufe auf das Modell aufgebracht, um die resultierenden viskosen Verformungen $\mathrm{zu}$ berechnen. Die Ergebnisse werden mit den Kriechverformungen des Referenzbetons unter dauerhafter Druckbeanspruchung ins Verhältnis gesetzt, um das kriechaffine Beanspruchungsniveau des zyklischen Beanspruchungsverlaufs $\mathrm{zu}$ bestimmen. Die Untersuchungen erfolgen für unterschiedliche Mittelspannungen, Spannungsamplituden und Belastungsfrequenzen des zyklischen Beanspruchungsverlaufs, wobei unterschiedliche Kriechtheorien betrachtet werden.

Auf Grundlage der rheologischen Modellvorstellung und dem kriechaffinen Beanspruchungsniveau können schlussendlich die elastischen, viskosen und thermischen Dehnungen eines ermüdungsbeanspruchten Betonkörpers beschrieben und bestimmt werden. Der schädigungsinduzierte Dehnungsanteil ergibt sich aus experimentell aufzuzeichnenden Gesamtverformungen abzüglich der zuvor genannten Dehnungsanteile entsprechend Gleichung 3.5 .

$$
\left|\varepsilon_{d}\right|=\left|\varepsilon_{\text {fat }}\right|-\left|\varepsilon_{e l}\right|-\left|\varepsilon_{c r}\right|+\left|\varepsilon_{t}\right|
$$

\subsubsection{Experimentelle Verifikation}

Um das kriechaffine Beanspruchungsniveau zu bestimmen und das additive Dehnungsmodell zu verifizieren, werden Ermüdungsversuche an dem zuvor genannten Referenzbeton durchgeführt. Als Beton soll ein höherfester Normalbeton untersucht werden, um einerseits als Bindeglied zwischen den Untersuchungen an normalfesten und hochfesten Betonen aus der Literatur zu dienen, aber auch, um den heutigen Betonanforderungen dynamisch hochbeanspruchter Bauwerke 
zu entsprechen. Die Dehnungen der Betonprobekörper werden dabei aufgezeichnet. Die einzelnen Dehnungskomponenten werden auf Grundlage der präsentierten Modellvorstellung bestimmt und ihre Anteile an den Gesamtverformungen dargestellt und diskutiert.

Die Ermüdungsversuche werden in zwei Versuchsserien durchgeführt. Die zu berücksichtigenden Oberspannungsniveaus der ersten Versuchsserie werden $\mathrm{zu} 80 \%, 70 \%$ und $60 \%$ der Betondruckfestigkeit gewählt. Die Versuche auf dem höchsten Oberspannungsniveau $\left(S_{o}=0,80\right)$ werden nach kurzen Versuchsdauern versagen und nur vernachlässigbare viskose Dehnungsanteile aufweisen. Die Versuche auf den geringeren Oberspannungsniveaus $\left(S_{o}=0,70\right.$ und 0,60 ) werden dagegen deutlich längere Versuchslaufzeiten und dementsprechend größere viskose Dehnungen aufweisen. Die Versuche werden bei Belastungsfrequenzen von $10 \mathrm{~Hz}$ und $1 \mathrm{~Hz}$ durchgeführt. Daraus resultieren wiederrum deutlich unterschiedliche Versuchslaufzeiten und dementsprechend stark unterschiedliche viskose Dehnungen.

Die Versuche der zweiten Serie werden bei einer Belastungsfrequenz von $10 \mathrm{~Hz}$ und auf Oberspannungsniveaus von $70 \%$ und $60 \%$ der Betondruckfestigkeit durchgeführt. Die Versuche der zweiten Serie unterscheiden sich von der ersten Serie durch ein deutlich höheres Betonalter, um einen alterungsbedingten Kriecheinfluss zu beurteilen. Demzufolge wird der Einfluss unterschiedlicher Belastungsfrequenzen auf das Verformungsverhalten im Rahmen der ersten Versuchsserie und der Einfluss unterschiedlicher Betonalter bei den $10 \mathrm{~Hz}$-Versuchen zwischen der ersten und zweiten Versuchsserie betrachtet, vgl. Tabelle 3.1.

Das Unterspannungsniveau wird bei allen Versuchen zu $5 \%$ der Betondruckfestigkeit gewählt, um den Fehler bei der Abschätzung der Dehnungsdifferenz zwischen Unterspannungsniveau und Entlastungsniveau zu minimieren. Durch die unterschiedlichen Belastungsfrequenzen treten unterschiedliche Probekörpererwärmungen und Temperaturdehnungen auf. Die Probekörpererwärmung wird messtechnisch erfasst, um die resultierenden Temperaturdehnungen abschätzen zu können.

Tabelle 3.1: Zu untersuchende Spannungsniveaus im Ermüdungsversuch

\begin{tabular}{|c|c|c|c|c|c|}
\hline \multirow{2}{*}{$\begin{array}{c}S_{o}=\sigma_{o} / f_{c} \\
{[-]}\end{array}$} & \multirow{2}{*}{$\begin{array}{c}S_{u}=\sigma_{u} / f_{c} \\
{[-]}\end{array}$} & \multirow{2}{*}{$\begin{array}{c}f_{p} \\
{[\mathrm{~Hz}]}\end{array}$} & \multicolumn{2}{|c|}{ Versuchsserie } & \\
\hline & & & I & II & \\
\hline 0,80 & \multirow{3}{*}{0,05} & \multirow{3}{*}{10} & $\checkmark$ & & \multirow{6}{*}{ 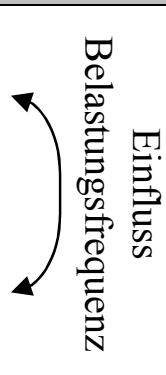 } \\
\hline 0,70 & & & $\checkmark$ & $\checkmark$ & \\
\hline 0,60 & & & $\checkmark$ & $\checkmark$ & \\
\hline 0,80 & \multirow{3}{*}{0,05} & \multirow{3}{*}{1} & $\checkmark$ & & \\
\hline 0,70 & & & $\checkmark$ & & \\
\hline 0,60 & & & $\checkmark$ & & \\
\hline & & & & lter & \\
\hline
\end{tabular}


Die Kriechversuche an dem Referenzbeton erfolgen auf Beanspruchungsniveaus von $40 \%, 50 \%$ und $60 \%$ der Betondruckfestigkeit. Die Dehnungen der Betonprobekörper werden während der Versuchsdurchführung kontinuierlich aufgezeichnet. Der Formalismus des Model Code 2010, zur Beschreibung von Kriechverformungen, wird an die aufgezeichneten Kriechverläufe adaptiert. Auf dieser Basis ist es möglich für beliebige Spannungsniveaus das Kriechverhalten des Referenzbetons zu prognostizieren. Anhand dessen werden die kriechaffinen Beanspruchungsniveaus, der im Ermüdungsversuch aufgebrachten zyklischen Beanspruchungsverläufe, bestimmt.

Die viskosen Verformungen der ermüdungsbeanspruchten Betonprobekörper können mithilfe der abgeleiteten kriechaffinen Beanspruchungsniveaus und dem adaptierten Formalismus des Model Code 2010 rechnerisch ermittelt werden. Die elastischen und thermischen Dehnungsanteile können anhand der Verformungs- und Temperaturmessungen näherungsweise ermittelt werden. Damit kann schlussendlich der schädigungsinduzierte Dehnungsanteil entsprechend Gleichung 3.5 bestimmt werden. Die einzelnen Dehnungsanteile werden analysiert und das additive Dehnungsmodell anhand dessen verifiziert. 


\section{$4 \quad$ Modellbildung und Ableitung kriechaffiner Beanspruchungsniveaus}

\subsection{Allgemeines}

Ziel dieses Kapitels ist die Modellentwicklung und die numerische Ermittlung kriechaffiner Beanspruchungsniveaus für ermüdungsbeanspruchte Betonprobekörper. Der Kriechverlauf und die Kriecheigenschaften des Betons werden analog zum Model Code 2010 angenommen. Dementsprechend wird bis zu einem Beanspruchungsniveau von $40 \%$ der Betondruckfestigkeit von linearem Kriechen ausgegangen, bei darüber hinausgehenden Beanspruchungsniveaus wird eine überproportionale Verformungszunahme unterstellt. Die Untersuchungen zum kriechaffinen Beanspruchungsniveau erfolgen für unterschiedliche Mittelspannungen, Spannungsamplituden und Belastungsfrequenzen im Ermüdungsversuch.
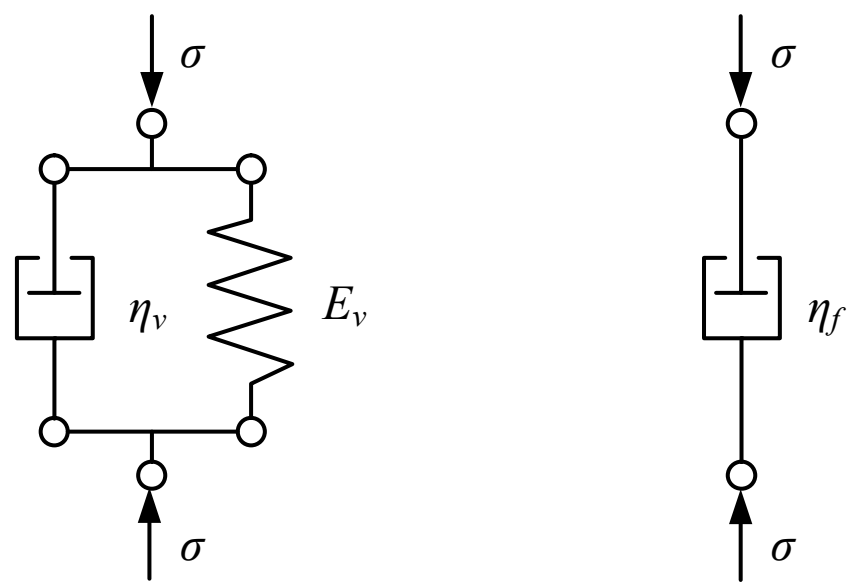

Abbildung 4.1: Modelle zur Betrachtung der viskosen Verformungen
Links: Reversibles Verformungsmodell Rechts: Irreversibles Verformungsmodell

In dem rheologischen Verformungsmodell aus Abbildung 3.4 werden die viskosen Verformungen über ein Burgers-Modell berücksichtigt. Dieses besteht aus einem Dämpferelement, das den irreversiblen Verformungsanteil des Betons abbildet, und einem in Reihe geschalteten KelvinKörper, der den reversiblen Verformungsanteil darstellt. Die prozentuale Gewichtung der reversiblen (verzögert-elastischen) Verformungen und der irreversiblen (Fließ-)Verformungen an den Gesamtkriechverformungen verschiebt sich mit zunehmendem Betonalter und zunehmender Beanspruchungshöhe unter einer konstanten Druckbeanspruchung. Da diese Verteilung voraussichtlich einen Einfluss auf das viskose Verformungsverhalten unter einer zyklischen Beanspruchung hat, werden die viskosen Verformungen einerseits als vollständig reversibel und andererseits als vollständig irreversibel betrachtet. In diesem Sinne werden zwei Modelle erarbeitet und analysiert. Das eine besteht aus einem Kelvin-Körper mit zeitinvarianten Parametern, um die viskosen Verformungen als verzögert-elastischen Verformungsprozess abzubilden. Das andere Modell fasst die viskosen Verformungen als plastische 
Verformungszunahme entsprechend der Theorie des Alterns (Dischinger-Ansatz) auf und besteht aus einem einzelnen Dämpferelement. Diese Modellansätze beschreiben im Grundsatz sehr einfache Kriechansätze, sie stellen aber zugleich auch die obere und untere Grenze möglicher Kriechansätze dar, vgl. Abschnitt 2.2.4. Die rheologischen Modelle sind in Abbildung 4.1 dargestellt.

Das Vorgehen zur Bestimmung des kriechaffinen Beanspruchungsniveaus wird in der Abbildung 4.2 schematisch gezeigt und im Folgenden beschrieben. Im ersten Schritt werden die mathematischen Gesetzmäßigkeiten der Modelle für eine dauerhafte Druckbeanspruchung aber auch für einen zyklischen Beanspruchungsverlauf entwickelt (Abschnitt 4.2). Die Modelle sollen sich wie zuvor genannt entsprechend der Theorie des Alterns (Dischinger-Ansatz) und der Theorie der linearen Viskoelastizität verhalten.

Die Modelle werden mithilfe einer makrogesteuerten Programmroutine numerisch umgesetzt. Das Verformungsverhalten dieser Modelle unter einer dauerhaften und konstanten Druckbeanspruchung wird an vorgegebene Kriechkurven adaptiert (Abschnitt 4.3). Hierzu werden die Feder- und Dämpfereigenschaften der Modelle für unterschiedliche Beanspruchungsniveaus per Best-FitAnalyse angepasst. Die vorgegebenen Kriechkurven entsprechen dem im Model Code 2010 beschriebenen Formalismus. Bis $\mathrm{zu}$ einem Druckspannungsniveau von $40 \%$ der Betondruckfestigkeit wird ein linearer Zusammenhang zwischen Kriechspannung und Kriechdehnung angenommen. Darüber hinaus wird eine überproportionale Verformungszunahme entsprechend Gleichung 2.5 und 2.6 unterstellt. Demzufolge werden bis zu einem Druckspannungsniveau von $40 \%$ der Betondruckfestigkeit konstante Federsteifigkeiten und Dämpferviskositäten erwartet. Im anschließenden Beanspruchungsbereich nehmen diese in Abhängigkeit von der Spannung ab, um die überproportionale Verformungszunahme abzubilden. Steifigkeit und Dämpfung sind somit spannungsabhängig zu formulieren. Auf dieser Grundlage können die vorgegebenen Kriechkurven unter einer konstanten Druckbeanspruchung mit beiden Modellen nachgerechnet werden. Dieses Vorgehen ist prinzipiell auch auf andere Kriechkurven (z. B. aus Versuchen), die nicht dem Formalismus des Model Code 2010 entsprechen, übertragbar.

Mithilfe der adaptierten Modelle werden die viskosen Verformungsverläufe infolge einer zyklischen Beanspruchung berechnet. Unterschiedliche Mittelspannungen, Spannungsamplituden und Belastungsfrequenzen werden untersucht. Die Verformungsverläufe werden den Kriechkurven unter einer dauerhaften und konstanten Druckbeanspruchung gegenübergestellt. Es wird das Kriechniveau unter der konstanten Beanspruchung gesucht, welches die gleichen Verformungen hervorruft, wie der zyklische Beanspruchungsverlauf. Ist dieses Kriechniveau gefunden, entspricht dieses dem kriechaffinen Beanspruchungsniveau des zyklischen Beanspruchungsverlaufs. Es wird erwartet, dass beim irreversiblen Modell größere viskose Verformungen auftreten als bei dem reversiblen Modell unter der zyklischen Beanspruchung. Die Ursache wird in der Rückverformbarkeit des reversiblen Modells während der Entlastungsphasen des zyklischen Beanspruchungsverlaufs gesehen. Es werden somit je Beanspruchungs- 
kombination zwei kriechaffine Beanspruchungsniveaus, eins für das irreversible Modell und eins für das reversible Modell, als Ergebnis erwartet.

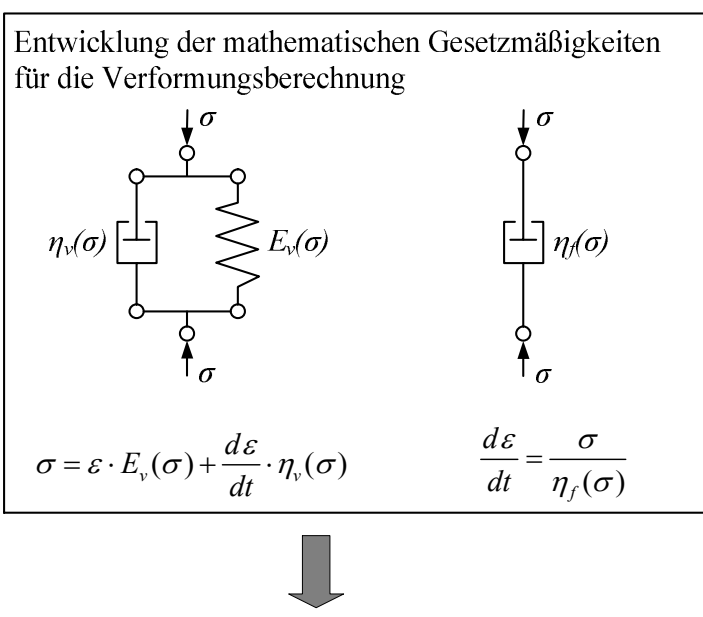

Bestimmung der Feder- und Dämpfereigenschaften per Best-Fit-Analyse
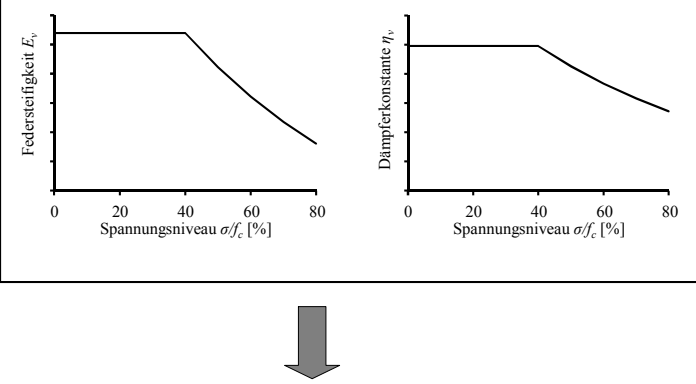

Berechnung der viskosen Verformungen infolge der zyklischen Beanspruchung
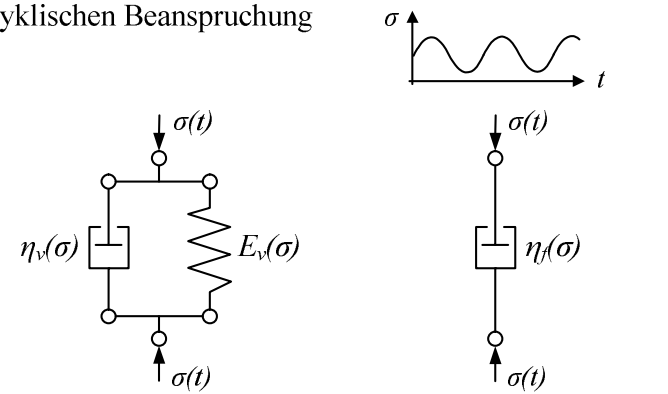

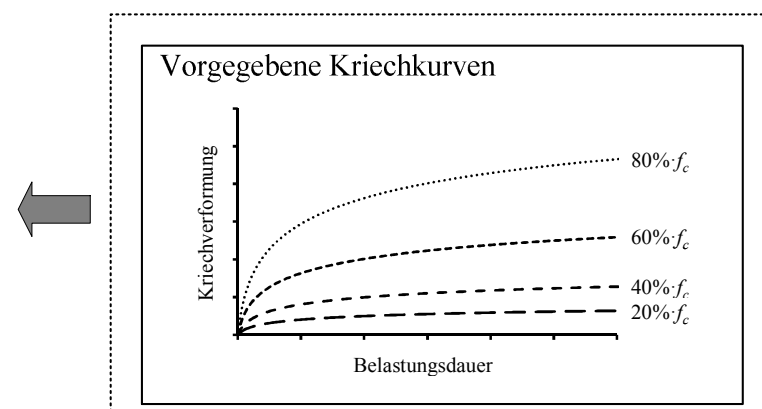

Viskose Verformungskurven infolge der zyklischen Beanspruchung
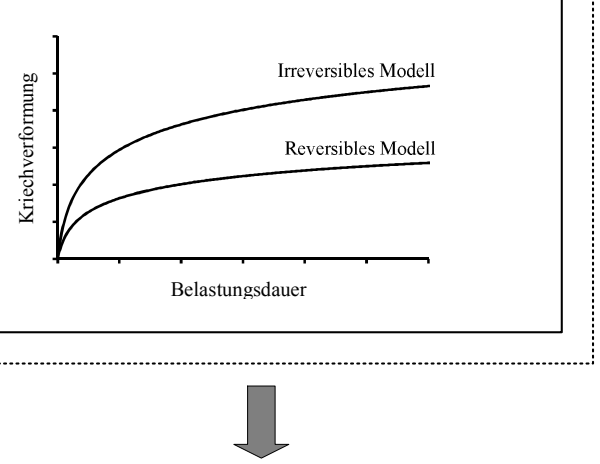

Best-Fit-Analyse zur Bestimmung der kriechaffinen Beanspruchungsniveaus

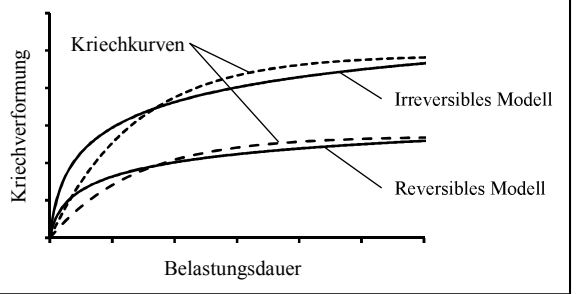




\subsection{Mathematische Modellentwicklung}

Das reversible Modell soll konform zur Theorie der linearen Viskoelastizität formuliert werden. Das irreversible Modell soll mit dem Dischinger-Ansatz korrespondieren. Die Modellansätze repräsentieren nicht den jüngsten Stand der Kriechforschung, die das Kriechen in der Form von „ausgefeilten“ Summations- oder Produktansätzen beschreiben. Die gewählten Ansätze ermöglichen aber zum einen die einfache Überprüfung und Plausibilisierung der Ergebnisse. Andererseits können diese Ansätze als obere und untere Modellierungsvariante des Betonkriechens erachtet werden. Neuere Ansätze betrachten das Kriechen als Mischform dieser beiden Theorien. Die Realität wird sich somit zwischen den gefunden Lösungen manifestieren.

Die Modelle werden mathematisch so formuliert und programmiertechnisch umgesetzt, dass damit die Verformungsverläufe unter einer dauerhaften und zyklischen Beanspruchung berechnet werden können. Wenn im Folgenden von einem zyklischen Beanspruchungsverlauf gesprochen wird, ist von einem sinusförmigen Spannungsverlauf auszugehen. Dieser wird über die Mittelspannung $\sigma_{m}$, die Spannungsamplitude $\sigma_{a}$ sowie die Belastungsfrequenz $f_{p}$ definiert. Die Phasenverschiebung des Beanspruchungsverlaufs wird über den Phasenwinkel $\psi$ gesteuert, wie in Gleichung 4.1 dargestellt.

$$
\sigma(t)=\sigma_{m}+\sigma_{a} \cdot \sin \left(f_{p} \cdot t+\psi\right)
$$

Falls von einem sinusförmigen Spannungsverlauf abgewichen wird, wird darauf speziell hingewiesen. Im Folgenden bezeichnet die Zeitvariable $t$ die Belastungsdauer des Betonkörpers. Das Betonalter bei Belastungsbeginn $t_{0}$ wird im Rahmen der Modellbildung formelmäßig nicht weiter betrachtet. Ermüdungsversuche dauern in der Regel nur wenige Stunden bis Tage. Dementsprechend kann die Nacherhärtung des Betons bzw. deren Einfluss auf die Betonfestigkeit und Betonsteifigkeit während der Versuchsdurchführung in den meisten Fällen vernachlässigt werden.

\subsubsection{Reversibles Modell}

Die Theorie der linearen Viskoelastizität setzt zeitinvariante Kriecheigenschaften voraus. Dementsprechend sind die Feder- und Dämpfereigenschaften des rheologischen Modells unabhängig von dem Betonalter und der Belastungsdauer zu formulieren. Die Verformungen des reversiblen Modells werden über einen Kelvin-Körper beschrieben. Die Verformungen des rheologischen Körpers ergeben sich aus dem Kräftegleichgewicht an dem Feder-Dämpfer-System. Die Feder- und Dämpfereigenschaften seien in Abhängigkeit von der Spannung über die Parameter $E_{v}(\sigma)$ und $\eta_{v}(\sigma)$ definiert. Wird zunächst von einer konstanten Druckbeanspruchung ausgegangen, sind die Federsteifigkeit und die Dämpferviskosität konstant. Es gilt $\sigma(t)=\sigma_{0}$, $E_{v}\left(\sigma_{0}\right)=E_{v, 0}$ und $\eta_{v}\left(\sigma_{0}\right)=\eta_{v, 0}$. Zu jedem Zeitpunkt muss ein Gleichgewicht zwischen den äußeren und inneren Spannungen bestehen. Es gilt: 


$$
\begin{gathered}
\sigma_{0}=\sigma_{\text {Feder }}(t)+\sigma_{\text {Dämpfer }}(t) \\
\Leftrightarrow \quad \sigma_{0}=\varepsilon_{c r}(t) \cdot E_{v, 0}+\frac{d \varepsilon_{c r}(t)}{d t} \cdot \eta_{v, 0}
\end{gathered}
$$

Hierin beschreibt $\varepsilon_{c r}(t)$ die eingetretene Kriechverformung zum Zeitpunkt $t$ und der Ausdruck $d \varepsilon_{c r}(t) / d t$ die Ableitung der Kriechverformung bzw. die Kriechgeschwindigkeit zum Zeitpunkt $t$. Aus der Gleichgewichtsbedingung ergibt sich eine Differentialgleichung 1. Ordnung, deren allgemeine Lösung in Gleichung 4.3 dargestellt ist.

$$
\varepsilon_{c r}(t)=C \cdot e^{\frac{E_{v, 0}}{\eta_{v, 0}} \cdot t}+\frac{\sigma_{0}}{E_{v, 0}}
$$

In dieser allgemeinen Lösung beschreibt $C$ eine Integrationskonstante. Wird die Beanspruchung zum Zeitpunkt $t_{0}=t=0$ aufgebracht, ergibt sich der Kriechverlauf zu:

$$
\varepsilon_{c r}(t)=\frac{\sigma_{0}}{E_{v, 0}} \cdot\left(1-e^{\frac{E_{v, 0}, t}{\eta_{v, 0}}}\right)
$$

Die in Gleichung 4.2 dargestellte Gleichgewichtsbedingung kann für einen zeitlich veränderlichen Beanspruchungsverlauf und spannungsabhängige Feder- und Dämpfereigenschaften nicht mehr analytisch geschlossen gelöst werden. In dem Falle sind die Verformungen iterativ zu ermitteln. Hierzu wird der Belastungsverlauf in $n$ Zeitschritte der Größe $\Delta \tau$ unterteilt. Die Gesamtverformung des Betons ergibt sich aus der Superposition der in den einzelnen Zeitschritten auftretenden Verformungen. Dieses Vorgehen entspricht dem Superpositionsprinzip aus Abschnitt 2.2.4 bei einer vertikalen Unterteilung des Spannungsverlaufs. Diese Superpositionsmethode ist anzuwenden, da ein nichtlinearer Zusammenhang zwischen Spannung und Dehnung vorliegt.

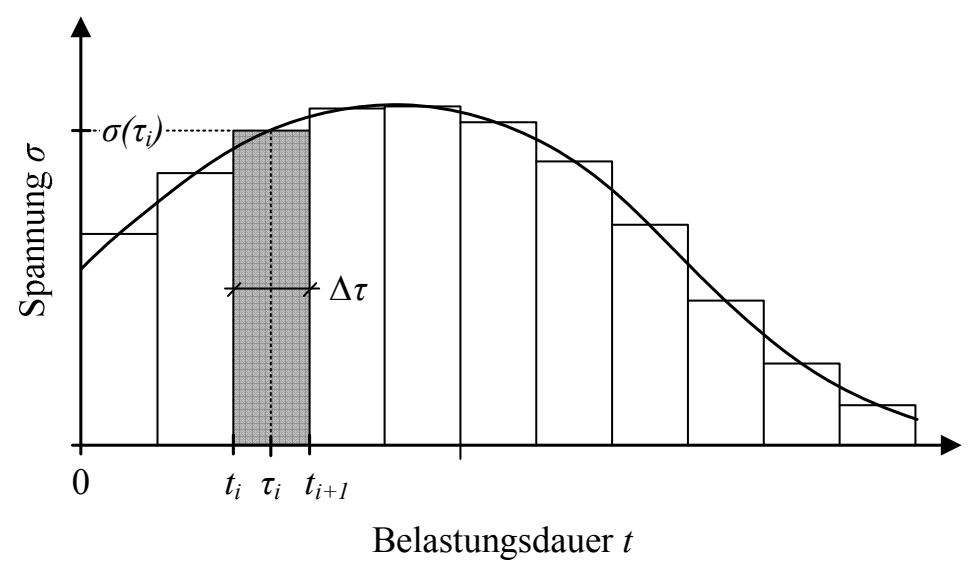

Abbildung 4.3: Superpositionsprinzip und Parameterdefinition

Innerhalb des $i$-ten Zeitschritts, in der Zeit von $t_{i}$ bis $t_{i+1}$, wird die Spannung als konstant angenommen. Sie entspricht der Spannung zum Zeitpunkt $\tau_{i}=t_{i}+\Delta \tau / 2$. Innerhalb des betrachteten Zeitschritts werden konstante Feder- und Dämpfereigenschaften angesetzt. Sie lauten für den $i$-ten Zeitschritt $E_{v, i}$ bzw. $\eta_{v, i}$ und werden für die mittlere Spannung $\sigma\left(\tau_{i}\right)$ ermittelt. 
Die Verformungszunahme innerhalb des betrachteten Zeitschritts wird auf Basis der in Gleichung 4.3 dargestellten allgemeinen Lösung der Differentialgleichung berechnet. Die Integrationskonstante $C$ lässt sich aus den Anfangsbedingungen des Zeitschritts bestimmen. Die Verformung zu Beginn des Zeitschritts entspricht der bis zu diesem Zeitpunkt eingetretenen Dehnung $\varepsilon_{c r}\left(t_{i}\right)$. Die mittlere Spannung des Zeitschritts lautet $\sigma\left(\tau_{i}\right)$. Damit ergibt sich die Integrationskonstante zu:

$$
C_{i}=\left(\varepsilon_{c r}\left(t_{i}\right)-\frac{\sigma\left(\tau_{i}\right)}{E_{v, i}}\right) \cdot e^{\frac{E_{v, i}}{\eta_{v, i}} \cdot t_{i}}
$$

Mithilfe der Integrationskonstante kann die Verformung am Ende des Zeitschritts zum Zeitpunkt $\varepsilon_{c r}\left(t_{i+1}\right)$ bestimmt werden zu:

$$
\varepsilon_{c r}\left(t_{i+1}\right)=C_{i} \cdot e^{\frac{E_{v, i} \cdot t_{i+1}}{\eta_{v i,}}}+\frac{\sigma\left(\tau_{i}\right)}{E_{v, i}}
$$

Damit stellt Gleichung 4.6 die Gesamtverformung des reversiblen Modells zum Zeitpunkt $t_{i+1}$ dar.

\subsubsection{Irreversibles Modell}

Das wesentliche Merkmal der Theorie des Alterns ist, dass der Einfluss des Betonalters und der zeitliche Verlauf der Kriechverformungen über die gleiche Funktion beschrieben werden. Damit ergeben sich für das irreversible Modell zeitvariante Materialparameter. In dem irreversiblen Modell werden die Kriechverformungen ausschließlich über ein Dämpferelement berücksichtigt. Der Newtonsche Dämpfer ist durch eine konstante Dämpfersteifigkeit definiert. Daraus resultiert in Abhängigkeit der Beanspruchung eine lineare Verformungszunahme mit der Zeit, vgl. Tabelle 2.1. Analog zu dem reversiblen Modell ist die Dämpferkonstante jedoch mit steigendem Spannungsniveau weicher werdend zu formulieren, um die Nichtlinearität des Betonkriechens zu berücksichtigen. Da die Verformungsgeschwindigkeit im Kriechversuch jedoch über die Versuchsdauer sukzessive abnimmt, ist die Dämpferkonstante nicht nur von der Spannung, sondern auch in Abhängigkeit von der Belastungsdauer zu definieren. Die Gleichung 4.7 stellt diesen mathematischen Zusammenhang dar.

$$
\frac{d \varepsilon_{c r}(t)}{d t}=\frac{\sigma(t)}{\eta_{f}(\sigma, t)}
$$

Um die Ergebnisse des irreversiblen und des reversiblen Modells miteinander vergleichen zu können, sind die Dämpfereigenschaften des irreversiblen Modells so zu wählen, dass unter einer dauerhaften und konstanten Druckbeanspruchung die gleichen Kriechverformungen prognostiziert werden, wie bei dem reversiblen Modell. Unter der Voraussetzung, dass der Kriechverlauf differenzierbar ist, kann entsprechend Gleichung 2.17 die Dämpferkonstante aus der Ableitung des Kehrwerts der Kriechverformung bestimmt werden. Die Kriechfunktion des Kelvin-Elements ist für eine konstante Druckbeanspruchung über die Gleichung 4.3 definiert. Wird diese in Gleichung 4.7 eingesetzt, resultiert daraus die Dämpferkonstante des irreversiblen Modells. 


$$
\begin{aligned}
\frac{d}{d t}\left[\frac{\sigma_{0}}{E_{v, 0}} \cdot\left(1-e^{-\frac{E_{v, 0}}{\eta_{v, 0}}} \cdot t\right)\right] & =\frac{\sigma_{0}}{\eta_{f}\left(\sigma_{0}, t\right)} \\
\Leftrightarrow \quad \frac{\sigma_{0}}{\eta_{v, 0}} \cdot e^{-\frac{E_{v, 0}}{\eta_{v, 0}} \cdot t} & =\frac{\sigma_{0}}{\eta_{f}\left(\sigma_{0}, t\right)} \\
\Leftrightarrow \quad \eta_{f}\left(\sigma_{0}, t\right) & =\eta_{v, 0} \cdot e^{\frac{E_{v, 0}}{\eta_{v, 0}} \cdot t}
\end{aligned}
$$

Die Berechnung der Verformungen für den zyklischen Beanspruchungsverlauf erfolgt wie zuvor durch vertikales Unterteilen des Spannungsverlaufs in $n$ Zeitschritte der Größe $\Delta \tau$. Die Superposition der in den einzelnen Zeitschritten auftretenden Verformungen ergibt die Gesamtverformung. Die Steifigkeiten $E_{v, i}$ und $\eta_{v, i}$ werden analog zum reversiblen Modell für die mittlere Spannung $\sigma\left(\tau_{i}\right)$ des $i$-ten Zeitschritts bestimmt. Die Verformungsgeschwindigkeit des irreversiblen Modells ergibt sich damit zu

$$
\frac{d \varepsilon_{c r}(t)}{d t}=\frac{\sigma\left(\tau_{i}\right)}{\eta_{v, i}} \cdot e^{-\frac{E_{v, i}}{\eta_{v, i}} t}
$$

und die Integration des Geschwindigkeitsverlaufs liefert die Verformung in dem betrachteten Zeitschritt:

$$
\begin{aligned}
& \varepsilon_{c r}\left(\Delta \tau_{i}\right)=\int_{t_{i}}^{t_{i+1}} \frac{\sigma\left(\tau_{i}\right)}{\eta_{v, i}} \cdot e^{-\frac{E_{v, i}}{\eta_{v, i}}} d t \\
& =\frac{\sigma\left(\tau_{i}\right)}{E_{v, i}} \cdot e^{-\frac{E_{v, i} \cdot t_{i+1}}{\eta_{v, i}}}-\frac{\sigma\left(\tau_{i}\right)}{E_{v, i}} \cdot e^{-\frac{E_{v, i}}{\eta_{v, i}} \cdot t_{i}} \\
& =\frac{\sigma\left(\tau_{i}\right)}{E_{v, i}} \cdot\left(e^{-\frac{E_{v, i} \cdot t_{t_{i+1}}}{\eta_{v, i}}}-e^{-\frac{E_{v, j} \cdot t_{i, i}}{\eta_{v, i}}}\right)
\end{aligned}
$$

Die Gesamtverformung ergibt sich aus der Summe der in den einzelnen Zeitschritten auftretenden Verformungen:

$$
\varepsilon_{c r}(t)=\sum_{i=1}^{n} \varepsilon_{c r}\left(\Delta \tau_{i}\right)
$$

Die Dämpferkonstante $\eta_{f}$ ist in Abhängigkeit von der Belastungsdauer $t$ formuliert. Die Belastungsdauer ist unter einer konstanten Druckbeanspruchung eindeutig definiert als die Zeitspanne, während der der Beton beansprucht wird. Wird der Beton jedoch nach einer ersten Belastungsphase vollständig entlastet und wieder neu belastet, stellt sich die Frage, ob die Zeit $t$ während dieser Entlastungsphase weiter läuft oder ob die Zeit bei Neubelastung wieder von null startet. Da bei der Theorie des Alterns der Einfluss der Betonalterung und der Kriechverlauf definitionsgemäß über die gleiche Funktion beschrieben werden, läuft die Zeit während der Entlastungsphase weiter. 
Sowohl das reversible als auch das irreversible Modell berücksichtigen die viskosen Verformungen über die gleiche Verformungsfunktion. Die zu wählenden Parameter der Modelle sind für beide Modelle gleich und unter einer konstanten und dauerhaften Belastung können mit beiden Modellen identische Kriechverläufe berechnet werden. Geringfügige Abweichungen können aus der summarischen Verformungsberechnung resultieren. Erst die Be- und Entlastungszyklen lassen eine Differenzierung der Ergebnisse erwarten, die ein Resultat der angenommen Kriechtheorien sind.

\subsection{Kriechkurven und Modellparameter}

Um die vorgestellten Modelle verwenden zu können, sind deren Verformungsverläufe an vorgegebene Kriechkurven zu adaptieren. Hierzu sind die Feder- und Dämpfereigenschaften anzupassen. Die Abbildung 4.4 stellt in diesem Sinne Kriechkurven (durchgezogene Linien) eines untersuchten Referenzbetons dar, dessen Eigenschaften und Versuchsrandbedingungen in Kapitel 5 naher erläutert werden. Die experimentell aufgezeichneten Kriechkurven wurden mit dem Formelapparat des Model Code 2010 nachgerechnet und gegenüber den experimentellen Untersuchungen um weitere Spannungsniveaus ergänzt. Eine Gegenüberstellung der experimentell und numerisch bestimmten Kriechverformungen können dem Abschnitt 5.2.6 bzw. dem Anhang A.3 entnommen werden. Da die zugehörigen Ermüdungsversuche an dem Referenzbeton mehrheitlich innerhalb weniger Stunden versagten, wurde eine Zeitspanne von 36 Stunden bzw. 1,5 Tagen für die Betrachtung der Kriechverformungen gewählt. Um eine gute Übereinstimmung zwischen den Kurvenverläufen nach Model Code 2010 und den experimentell aufgezeichneten Kriechkurven $\mathrm{zu}$ erreichen, musste das rechnerische Betonalter bei Belastungsbeginn modifiziert werden, da die Kriechverläufe nach Model Code 2010 andernfalls die gemessenen Kriechverformungen unterschätzt hätten. Das modifizierte Belastungsalter $t_{0}{ }^{*}=5,2$ Tagen steht für ein reales Betonalter von $t_{0}=62,5$ Tagen und das modifizierte Alter $t_{0}{ }^{*}=6,9$ Tagen für eine reales Alter von $t_{0}=140,5$ Tagen des Referenzbetons. Die genannten realen Betonalter entsprechen den mittleren Betonaltern bei Durchführung der Ermüdungsversuchsserien I und II, vgl. Abschnitt 3.3.2. Die Umrechnung der Betonalter erfolgte nach Gleichung 5.3. Es sei darauf hingewiesen, dass der Model Code 2010 die Anwendbarkeit des Formelapparates zur Berechnung viskoser Verformungen auf eine maximale Kriechspannung von $60 \%$ der Betondruckfestigkeit begrenzt. In den Untersuchungen wurden die Gleichungen auch bis zu einem Spannungsniveau von $80 \%$ der Betondruckfestigkeit angewandt.

Des Weiteren werden in der Abbildung 4.4 die berechneten Kriechverläufe mit den vorgestellten, rheologischen Modellen dargestellt (Punkte). Die Feder- und Dämpfersteifigkeiten wurden entsprechend Abbildung 4.5 gewählt. Beide Modelle (reversibel und irreversibel) liefern unter Vorgabe einer konstanten Beanspruchung die gleichen Verformungen. Insgesamt lässt sich erkennen, dass bis zu einem Beanspruchungsniveau von $60 \%$ der Betondruckfestigkeit eine gute Übereinstimmung zwischen den vorgegebenen Kriechverläufen nach Model Code 2010 (durchgezogene Linien) und den rechnerisch ermittelten Kriechverläufen (Punkte) besteht. Darüber hinausgehende Beanspruchungsniveaus können weniger gut abgebildet werden. Die 
starken Kriechverformungen des Betons zu Beginn der Versuchsdurchführung können unter Ansatz der vorausgesetzten zeitunabhängigen Feder- und Dämpfereigenschaften des reversiblen Modells nur mäßig abgebildet werden. Vergleichsrechnungen mit dem Ansatz von (Pfefferle, 1971) ermöglichten die Abbildung der Kriechkurven nach Model Code 2010 nahezu exakt. Der Dämpfer des reversiblen Modells ist hierzu in der Form von

$$
\eta_{v}(\sigma, t)=2 \cdot \eta(\sigma) \cdot \sqrt{t}
$$

$\mathrm{zu}$ formulieren. Bei Berücksichtigung der Beanspruchungsdauer $t$ in der Dämpferkonstante bedeutet dies aber auch, dass die Dämpfersteifigkeit mit zunehmender Beanspruchungsdauer immer weiter zunimmt und die Kriechverformungen immer langsamer auftreten. Dementsprechend verlangsamt sich auch das Rückkriechen bei der gedanklichen Entlastung des Betonkörpers zunehmend, sodass zwar noch ein visko-elastisches Verhalten bei dem reversiblen Modell vorliegt. Die beabsichtigte Grenzwertbetrachtung hinsichtlich eines idealen viskoelastischen Verhaltens wäre damit aber nicht mehr gegeben.
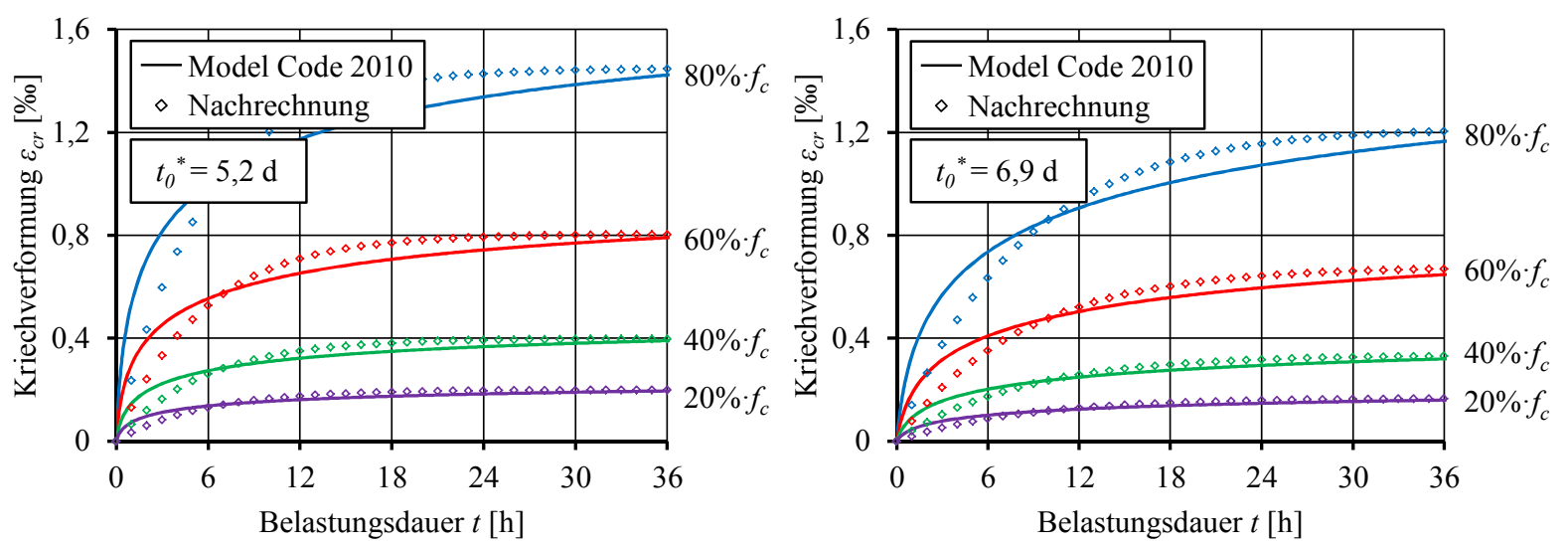

Abbildung 4.4: Kriechverläufe nach Model Code 2010 (durchgezogene Linien) und Nachrechnung mit dem reversiblen und irreversiblen Modell (Punkte)

Die Bestimmung der Feder- und Dämpfersteifigkeiten erfolgte über eine Best-Fit-Analyse. Hierzu wurde die Methode der kleinsten Fehlerquadrate herangezogen. Die Steifigkeiten der Feder und des Dämpfers wurden iterativ angepasst bis die Quadratsumme der Abweichungen zwischen dem vorgegebenen Kriechverlauf und dem berechnetem Verformungsverlauf ein Minimum annahmen, vgl. Gleichung 4.13.

$$
F=\sum_{i=i}^{m}\left(\varepsilon_{c r, i}^{M C 2010}-\varepsilon_{c r, i}^{\text {Model }}\right)^{2} \Rightarrow \text { Minimum }
$$

Dieses Vorgehen wurde für die unterschiedlichen Spannungsniveaus durchgeführt. Es resultierten die in Abbildung 4.5 dargestellten Feder- und Dämpfereigenschaften für die unterschiedlichen Betonalter. Bis zu einem Beanspruchungsniveau von $40 \%$ der Betondruckfestigkeit ergaben sich für die Feder- und Dämpfereigenschaften konstante Werte. Dieses Ergebnis resultiert aus dem Kriechansatz des Model Code 2010. In diesem wird bis zu dem genannten Beanspruchungsniveau ein linearer Zusammenhang zwischen Kriechverformung und Kriechspannung angenommen. 
Bei Beanspruchungen oberhalb von $40 \%$ der Betondruckfestigkeit werden die nichtlinearen Kriecheigenschaften über den Faktor $k_{\sigma}$ in Gleichung 2.6 berücksichtigt. Entsprechend diesem Faktor nehmen die Federsteifigkeit und die Dämpferkonstante in Abhängigkeit des Beanspruchungsniveaus ab. Der Verlauf der Feder- und Dämpfereigenschaften wurde dementsprechend in der folgenden Form in die rheologischen Modelle implementiert:

$$
\begin{aligned}
& E_{v}(\sigma)=E_{\text {lin }} / k_{\sigma} \text { und } \quad \eta_{v}(\sigma)=\eta_{\text {lin }} / k_{\sigma} \\
& \text { mit } \quad k_{\sigma}=e^{1,5 \cdot\left(\frac{\sigma}{f_{c}}-0,4\right)} \geq 1,0 \\
& E_{\text {lin }}, \eta_{\text {lin }} \quad \text { Feder- und Dämpfersteifigkeit im } \\
&
\end{aligned}
$$
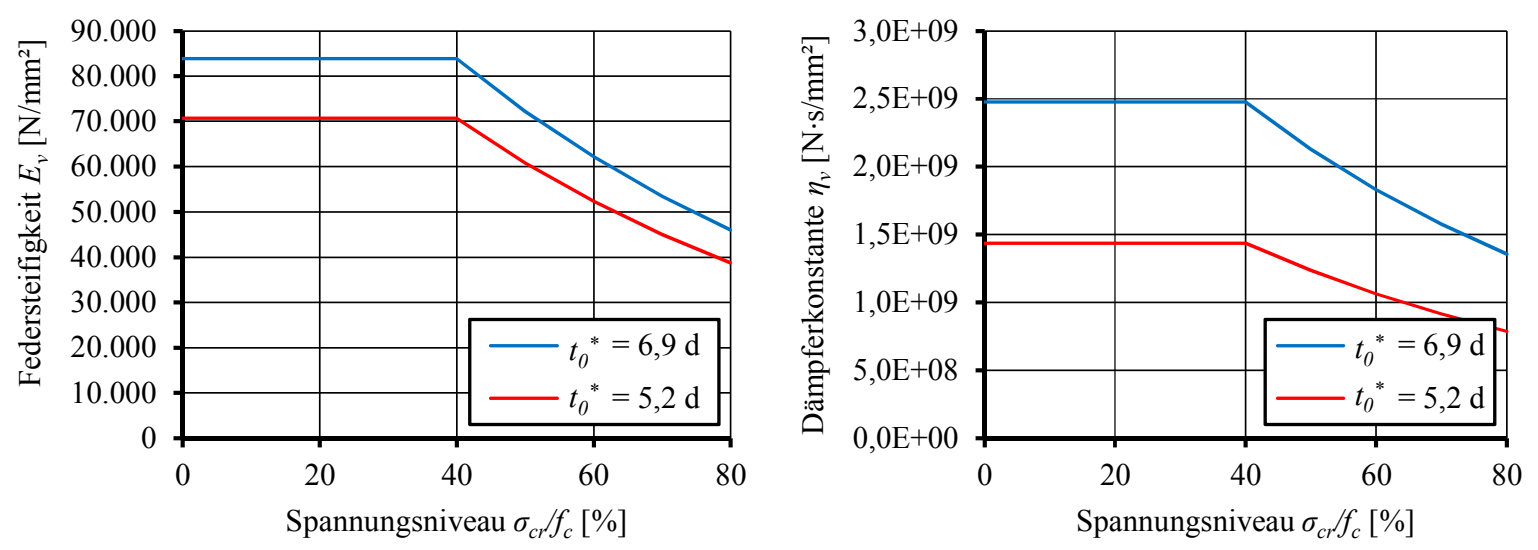

Abbildung 4.5: Feder- und Dämpfereigenschaften in Abhängigkeit des Spannungsniveaus und des Betonalters

\subsection{Modellverhalten unter variabler Beanspruchung}

Der Unterschied zwischen dem visko-elastischen und dem visko-plastischen Modell zeigt sich erst bei Entlastung nach einer vorhergehenden Druckbeanspruchung. Die Verformungen des visko-plastischen Modells bleiben nach der Entlastung im Dämpfer gespeichert, während sich der visko-elastische Kelvin-Körper in Richtung seiner Ausgangslage zurück verformt. Das reale Materialverhalten von Beton liegt zwischen diesen beiden Modellvorstellungen. Die prozentuale Verteilung zwischen visko-elastischen und visko-plastischen Verformungen verändert sich mit dem Belastungsalter, der Belastungsdauer und dem Alter bei der Entlastung. Die Ergebnisse der Modelle sind wie zuvor genannt im Sinne einer Grenzwertbetrachtung aufzufassen.

Die Abbildung 4.6 stellt die Verformungen des reversiblen und des irreversiblen Modells bei einer zeitlich begrenzten Beanspruchung dar. Das Beanspruchungsniveau liegt bei $60 \%$ der Betondruckfestigkeit für eine Beanspruchungsdauer von 18 Stunden. Anschließend wird der Betonkörper gedanklich entlastet und das Verformungsverhalten der Modelle für weitere 18 Stunden dargestellt. Im Weiteren sind die Kriechverformungen der Modelle unter einer zeitlich unbegrenzten Beanspruchung bei einem Beanspruchungsniveau von $60 \%$ der Betondruckfestigkeit dargestellt. Während der Belastungsphase ergeben sich für beide Modelle die gleichen Kriechverformungen. Nach der Entlastung verharren die Verformungen des 
irreversiblen Modells auf dem erreichten Niveau. Bei dem reversiblen Modell führt die Entlastung zu einer Rückverformung des Betonkörpers, die sich asymptotisch der Abszisse nähert. Nach der dargestellten 18-stündigen Entlastungsphase hat sich der Beton noch nicht wieder vollständig in seine Ausgangslage zurück verformt. Die Rückverformung läuft damit langsamer ab als die Kriechverformung während der Belastungsphase. In der Belastungsphase stehen die Feder- und Dämpferspannung im Gleichgewicht mit der äußeren Druckbeanspruchung. Zu Beginn der Belastungsphase lastet die gesamte äußere Druckspannung auf dem Dämpfer, woraus eine Verformungsgeschwindigkeit der Größe $\sigma_{c r} / \eta_{v}\left(\sigma_{c r}\right)$ resultiert. Mit zunehmenden Verformungen nehmen die Spannungen in der Feder zu und im Dämpfer ab. Dementsprechend nimmt auch die Verformungsgeschwindigkeit ab. Am Ende der 18-stündigen Belastungsphase sind die Kriechdehnungen noch nicht abgeschlossen, sodass noch ein Teil der äußeren Beanspruchung auf dem Dämpfer lastet. Folglich ist die Federspannung kleiner als die äußere Beanspruchung $\left(\sigma_{F e d e r}<\sigma_{c r}\right)$. Nach der Entlastung wird die äußere Spannung zu null. Die Federspannung und die Dämpferspannung müssen fortan im Gleichgewicht stehen. Da die Federspannung kleiner ist als die aufgebrachte Spannung bei Belastungsbeginn folgt daraus auch eine kleinere Verformungsgeschwindigkeit mit $\sigma_{\mathrm{Feder}} / \eta_{v}(\sigma=0)$ als zu Belastungsbeginn. Des Weiteren werden die Feder- und Dämpfereigenschaften in Abhängigkeit der vorherrschenden äußeren Spannung bestimmt. Während der Belastungsphase ist die Dämpfersteifigkeit, die verantwortlich ist für den zeitlich verzögerten Verlauf der Verformungsentwicklung, kleiner als in der Entlastungsphase. Daraus resultiert, dass das Rückkriechen langsamer abläuft als das Kriechen in der Belastungsphase.

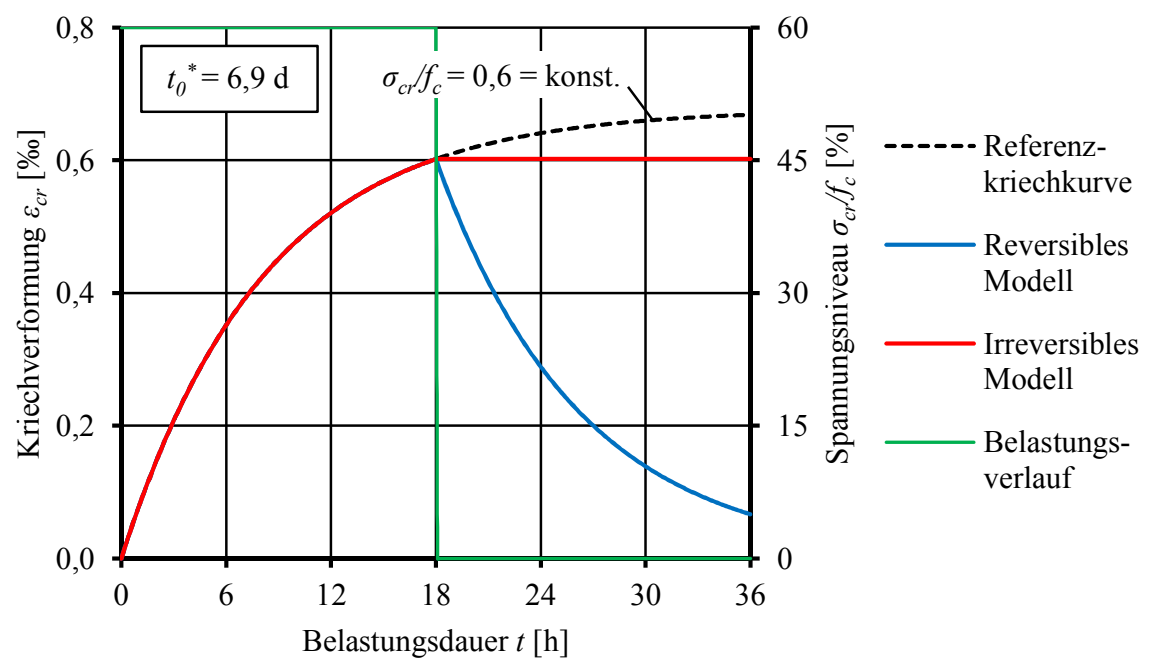

Abbildung 4.6: Verformungen des reversiblen und irreversiblen Modells unter einer zeitlich begrenzten Beanspruchung

Die Verformungen des reversiblen und des irreversiblen Modells unter einer rechteckförmigen, periodischen Beanspruchung werden in Abbildung 4.7 dargestellt. Das Oberspannungsniveau liegt bei $60 \%$ und das Unterspannungsniveau bei $0 \%$ der Betondruckfestigkeit, die Beanspruchungsdauer je Rechteck-Impuls beträgt eine Stunde. In der Grafik sind neben den Verformungsverläufen des reversiblen und des irreversiblen Modells auch die Kriechkurven für 
eine konstante Druckbeanspruchung auf dem Mittelspannungsniveau $\left(0,3 \cdot f_{c}\right)$ und dem Oberspannungsniveau $\left(0,6 \cdot f_{c}\right)$ des periodischen Verlaufs dargestellt. Bei dem irreversiblen Modell nehmen die Verformungen in den Belastungsphasen stufenförmig zu. Bei dem reversiblen Modell findet während der Entlastungsphasen ein Rückkriechen des Betons statt, wobei in den Belastungsphasen größere Kriechverformungen auftreten als bei dem irreversiblen Modell. Es resultiert ein sägezahnförmiger Verformungsverlauf. Beide Modelle beschreiben im Mittel den gleichen Verformungsverlauf. Dieser liegt zwischen den Kriechkurven des zugehörigen Mittelspannungsniveaus von $30 \%$ der Betondruckfestigkeit und des zugehörigen Oberspannungsniveaus von $60 \%$ der Betondruckfestigkeit.
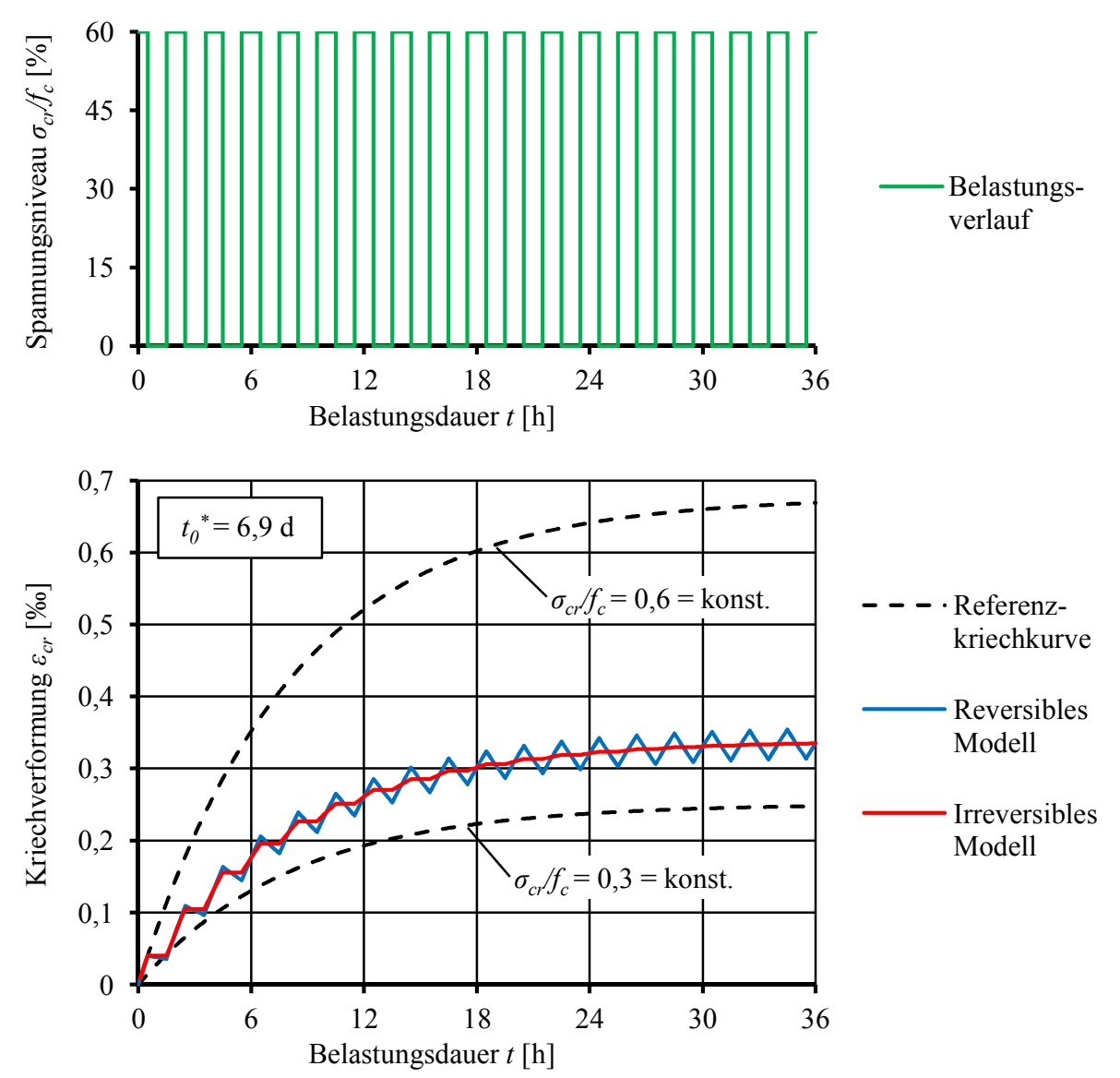

Abbildung 4.7: Belastungsverlauf (oben) und Verformungsverlauf des reversiblen und irreversiblen Modells (unten)
infolge einer periodischen, rechteckförmigen Belastung

Zunächst soll der Verformungsverlauf des irreversiblen Modells im Detail betrachtet werden. Die Verformungszunahme in den Belastungsphasen ergibt sich aus der vertikalen Verschiebung der Kriechkurve des Oberspannungsniveaus $\left(\sigma_{c r}=0,6 \cdot f_{c}\right)$, vgl. Abbildung 4.8. Diese Eigenschaft entspricht der Theorie des Alterns bzw. dem Dischinger-Ansatz, vgl. Abbildung 2.11 (links). Mit zunehmender Belastungsdauer flachen die Kriechkurve unter der dauerhaften Belastung und folglich auch die Verformungskurve unter dem periodischen Beanspruchungsverlauf immer weiter ab. In den Entlastungsphasen verharren die Verformungen auf dem erreichten Verformungsniveau. 


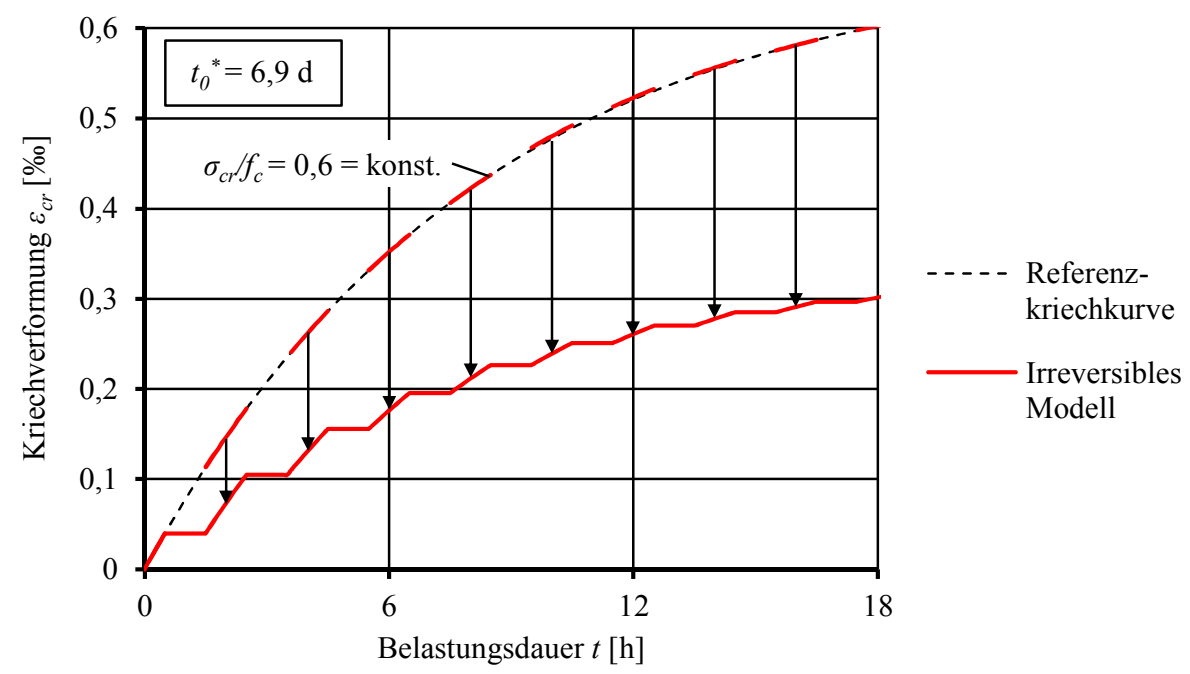

Abbildung 4.8: Verformungsverlauf des irreversiblen Modells im Detail

Durch die Wahl eines periodischen, rechteckförmigen Beanspruchungsverlaufs mit einem Unterspannungsniveau von $0 \%$ der Betondruckfestigkeit sind die auftretenden Verformungen halb so groß wie die Verformungen unter der dauerhaften Beanspruchung, da der Beton auch nur während der Hälfte der Versuchsdauer tatsächlich beansprucht wird. Diese Näherung ist gültig solange die Be- und Entlastungsphasen im Vergleich zur zeitlichen Entwicklung der Kriechverformungen ausreichend kurz sind. Wäre das Unterspannungsniveau abweichend von der Darstellung ungleich null, würde der Beton in diesen Phasen entsprechend dem zugehörigen Lastniveau weiter kriechen. Dementsprechend würden in diesen Phasen auf dem Unterspannungsniveau Verformungen auftreten, die wiederum halb so groß wären, wie die Kriechverformungen auf diesem Niveau bei einer dauerhaften Beanspruchung. Damit ergibt sich der viskose Verformungsverlauf unter der periodischen Beanspruchung zu:

$$
\varepsilon_{c r}\left(\sigma_{c r}, t\right)=\frac{1}{2} \cdot \varepsilon_{c r}\left(\sigma_{0}, t\right)+\frac{1}{2} \cdot \varepsilon_{c r}\left(\sigma_{u}, t\right)
$$

Die Variable $\sigma_{c r}$ stellt in diesem Zusammenhang die kriechaffine Spannung dar; die Variablen $\sigma_{o}$ und $\sigma_{u}$ die Ober- und die Unterspannung des periodischen, rechteckförmigen Beanspruchungsverlaufs. Unter der kriechaffinen Spannung treten bei einer kontinuierlichen Beanspruchung die gleichen viskosen Verformungen auf wie infolge des periodischen Beanspruchungsverlaufs. Werden die Verformungsverläufe durch den Ansatz aus Gleichung 2.6 ersetzt, ergibt sich das kriechaffine Beanspruchungsniveau der periodischen Beanspruchung zu:

$$
\begin{aligned}
& \sigma_{c r} \cdot J(t) \cdot k\left(\sigma_{c r}\right)=\frac{1}{2} \cdot \sigma_{o} \cdot J(t) \cdot k\left(\sigma_{o}\right)+\frac{1}{2} \cdot \sigma_{u} \cdot J(t) \cdot k\left(\sigma_{u}\right) \\
& \Leftrightarrow \quad \sigma_{c r} \cdot k\left(\sigma_{c r}\right)=\frac{1}{2} \cdot\left[\sigma_{o} \cdot k\left(\sigma_{o}\right)+\sigma_{u} \cdot k\left(\sigma_{u}\right)\right]
\end{aligned}
$$

Für den zuvor dargestellten rechteckförmigen Beanspruchungsverlauf mit $\sigma_{o}=0,6 \cdot f_{c}$ und $\sigma_{u}=0 \cdot f_{c}$ folgt: 


$$
\begin{array}{rrr} 
& \sigma_{c r} \cdot k\left(\sigma_{c r}\right)=\frac{1}{2} \cdot\left[0,6 \cdot f_{c} \cdot k\left(0,6 \cdot f_{c}\right)+0 \cdot f_{c} \cdot k\left(0 \cdot f_{c}\right)\right] \\
\Leftrightarrow & \sigma_{c r} \cdot k\left(\sigma_{c r}\right)= & \frac{1}{2} \cdot\left[0,6 \cdot f_{c} \cdot 1,349+0\right] \\
\Leftrightarrow & \sigma_{c r} \cdot k\left(\sigma_{c r}\right)= & 0,4047 \cdot f_{c}
\end{array}
$$

Die Abbildung 4.9 stellt den Einfluss des Kriechspannungsniveaus auf das Ergebnis der Kriechverformungen dar. Das Ergebnis folgt aus Gleichung 2.6 ausgewertet mit $J\left(t, t_{0}\right)=1$. Bis zu einem Beanspruchungsniveau von $40 \%$ der Betondruckfestigkeit ist dieser Einfluss zunächst linear. Im anschließenden Bereich nimmt dieser überproportional zu. Anhand dieser Darstellung ergibt sich das kriechaffine Beanspruchungsniveau für den betrachteten, rechteckförmigen Beanspruchungsverlauf zu etwa $0,4 \cdot f_{c}$. Damit ist das kriechaffine Beanspruchungsniveau für das irreversible Modell bei einer periodischen, rechteckförmigen Beanspruchung eindeutig definiert. Wie zu erkennen ist, ist die Komplianzfunktion $J(t)$ nicht in der Formel enthalten. Das kriechaffine Beanspruchungsniveau gilt somit unabhängig vom tatsächlichen Kriechverlauf. Allein die spannungsabhängige Verformungszunahme zwischen linearem und nichtlinearem Kriechen ist ausschlaggebend für das kriechaffine Beanspruchungsniveau. Des Weiteren ist der Zusammenhang unabhängig von der Belastungsfrequenz.

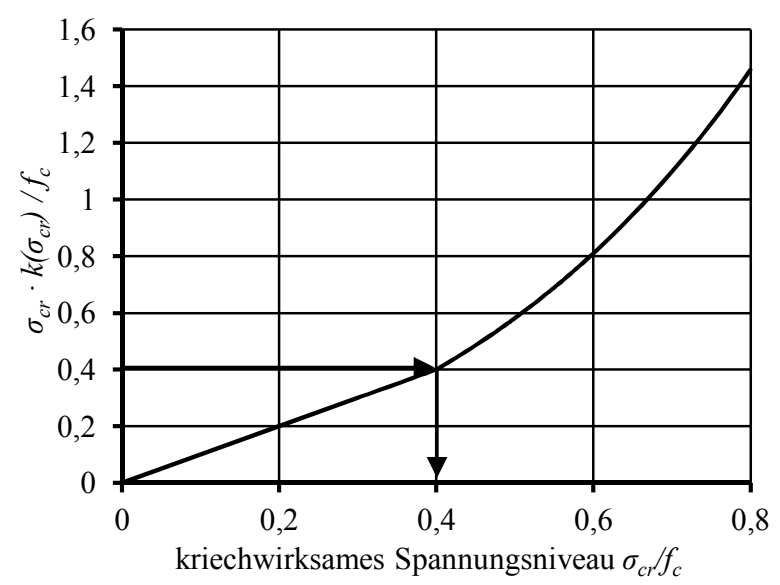

Abbildung 4.9: Einfluss des Spannungsniveaus auf die Kriechverformung

Die Abbildung 4.10 stellt den Verformungsverlauf des reversiblen Modells dar. Der Verformungsverlauf des reversiblen Modells lässt sich in Anlehnung an Abbildung 2.11 (rechts) aus der horizontalen Verschiebung der Kriechkurve des Oberspannungsniveaus erzeugen. Wie zuvor erläutert, nehmen die Verformungen des reversiblen Modells in den Belastungsphasen stärker zu als bei dem irreversiblen Modell. Die Ursache für dieses Verhalten liegt in dem Rückkriechen des Systems. In den Entlastungsphasen nimmt die Spannung in der Feder durch das Rückkriechen ab. Wird der Beton anschließend wieder belastet, ruht auf dem Dämpfer die äußere Spannung abzüglich der noch in der Feder gespeicherten Spannung. Dadurch ist die Dämpferspannung zu Beginn des Lastimpulses größer, als hätte kein Rückkriechen stattgefunden. Dementsprechend sind die Verformungsgeschwindigkeiten und die Kriechverformungen in den Belastungsphasen des reversiblen Modells größer als bei dem irreversiblen Modell. 


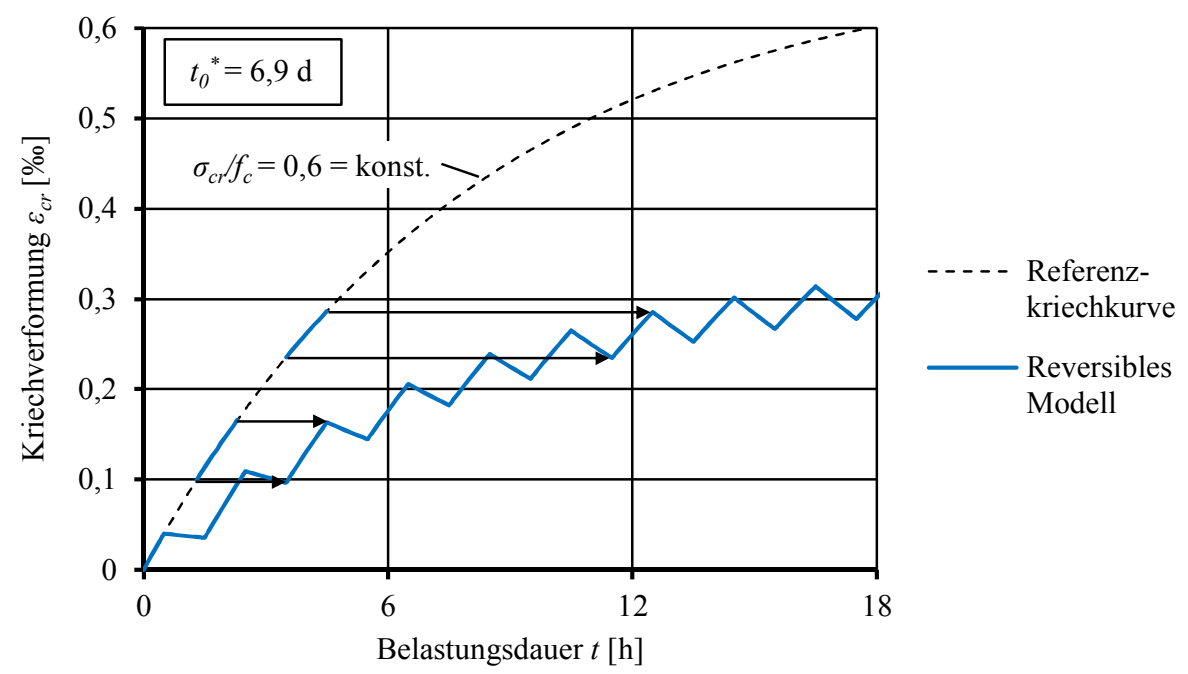

Abbildung 4.10: Verformungsverlauf des reversiblen Modells im Detail

Interessanterweise stellen sich bei dem reversiblen Modell die gleichen Verformungen ein wie bei dem irreversiblen Modell. Eigentlich wurde erwartet, dass das reversible Modell infolge des Rückkriechens kleinere Verformungen liefert als das irreversible Modell. Mit fortschreitender zyklischer Beanspruchung verharren die Verformungen auf einem Niveau, bei dem das Kriechen unter Last genauso groß ist wie das Rückkriechen in der Entlastungsphase. $\mathrm{Zu}$ diesem Zeitpunkt muss die Kriechgeschwindigkeit in den Be- und Entlastungsphasen im Mittel betragsmäßig gleich groß sein. Es gilt:

$$
\frac{d \varepsilon_{c r}\left(\sigma_{o}, t \rightarrow \infty\right)}{d t}=-\frac{d \varepsilon_{c r}\left(\sigma_{u}, t \rightarrow \infty\right)}{d t}
$$

Die Dehngeschwindigkeit entspricht dem Quotienten aus Dämpferspannung und Dämpfersteifigkeit. Dabei ergibt sich die Dämpferspannung aus der Differenz von äußerer Spannung und der in der Feder enthaltenen Spannung. Es gilt somit:

$$
\begin{array}{rlll} 
& \frac{\sigma_{\text {Dämpfer }, o}}{\eta_{v}\left(\sigma_{o}\right)} & = & -\frac{\sigma_{\text {Dämpfer }, u}}{\eta_{v}\left(\sigma_{u}\right)} \\
\Leftrightarrow \quad \frac{\sigma_{o}-\sigma_{\text {Feder }, o}}{\eta_{v}\left(\sigma_{o}\right)} & = & -\frac{\sigma_{u}-\sigma_{\text {Feder }, u}}{\eta_{v}\left(\sigma_{u}\right)} \\
\Leftrightarrow \quad \frac{\sigma_{o}-\sigma_{\text {Feder }, o}}{\eta_{\text {lin }}} & = & -\frac{\sigma_{u}-\sigma_{\text {Feder }, u}}{\frac{\eta_{\text {lin }}}{k\left(\sigma_{o}\right)}} \\
\Leftrightarrow \sigma_{o} \cdot k\left(\sigma_{o}\right)+\sigma_{u} \cdot k\left(\sigma_{u}\right) & =\sigma_{\text {Feder }, u} \cdot k\left(\sigma_{u}\right)+\sigma_{\text {Feder }, o} \cdot k\left(\sigma_{o}\right)
\end{array}
$$

Die Dehnungen bei Erreichen des Gleichgewichtszustandes sind während der Be- und Entlastungsphase im Mittel gleich. Es kommt zu keinen weiteren Verformungen. Die Dehnung entspricht dem Endkriechwert der periodischen Beanspruchung. Er wird folgend mit $\varepsilon_{c r}$ bezeichnet. Die Spannungen in der Feder können damit durch das Produkt aus Dehnung und Federsteifigkeit ersetzt werden. 


$$
\begin{aligned}
& \Leftrightarrow \sigma_{o} \cdot k\left(\sigma_{o}\right)+\sigma_{u} \cdot k\left(\sigma_{u}\right)=\varepsilon_{c r} \cdot \frac{E_{\text {lin }}}{k\left(\sigma_{u}\right)} \cdot k\left(\sigma_{u}\right)+\varepsilon_{c r} \cdot \frac{E_{\text {lin }}}{k\left(\sigma_{o}\right)} \cdot k\left(\sigma_{o}\right) \\
& \Leftrightarrow \sigma_{o} \cdot k\left(\sigma_{o}\right)+\sigma_{u} \cdot k\left(\sigma_{u}\right)=2 \cdot \varepsilon_{c r} \cdot E_{\text {lin }}
\end{aligned}
$$

Die Kriechdehnung entspricht der Dehnung, die unter dem kriechaffinen Beanspruchungsniveau auftritt. Damit gilt für die Endkriechdehnung wiederum:

$$
\varepsilon_{c r}=\frac{\sigma_{c r}}{E_{c r}}=\frac{\sigma_{c r} \cdot k\left(\sigma_{c r}\right)}{E_{l i n}}
$$

Daraus folgt:

$$
k\left(\sigma_{c r}\right) \cdot \sigma_{c r}=\frac{1}{2} \cdot\left[\sigma_{o} \cdot k\left(\sigma_{o}\right)+\sigma_{u} \cdot k\left(\sigma_{u}\right)\right]
$$

Die Gleichung 4.22 entspricht damit Gleichung 4.16. Abweichend von der ursprünglichen Annahme ergibt sich für das reversible und das irreversible Modell das gleiche kriechaffine Beanspruchungsniveau unter einer periodischen, rechteckförmigen Beanspruchung. Dieses ist allein von der proportionalen Beziehung zwischen Kriechspannung und Kriechdehnung abhängig. Die Kriechfunktion und auch die Belastungsfrequenz haben keinen Einfluss auf das kriechaffine Niveau.

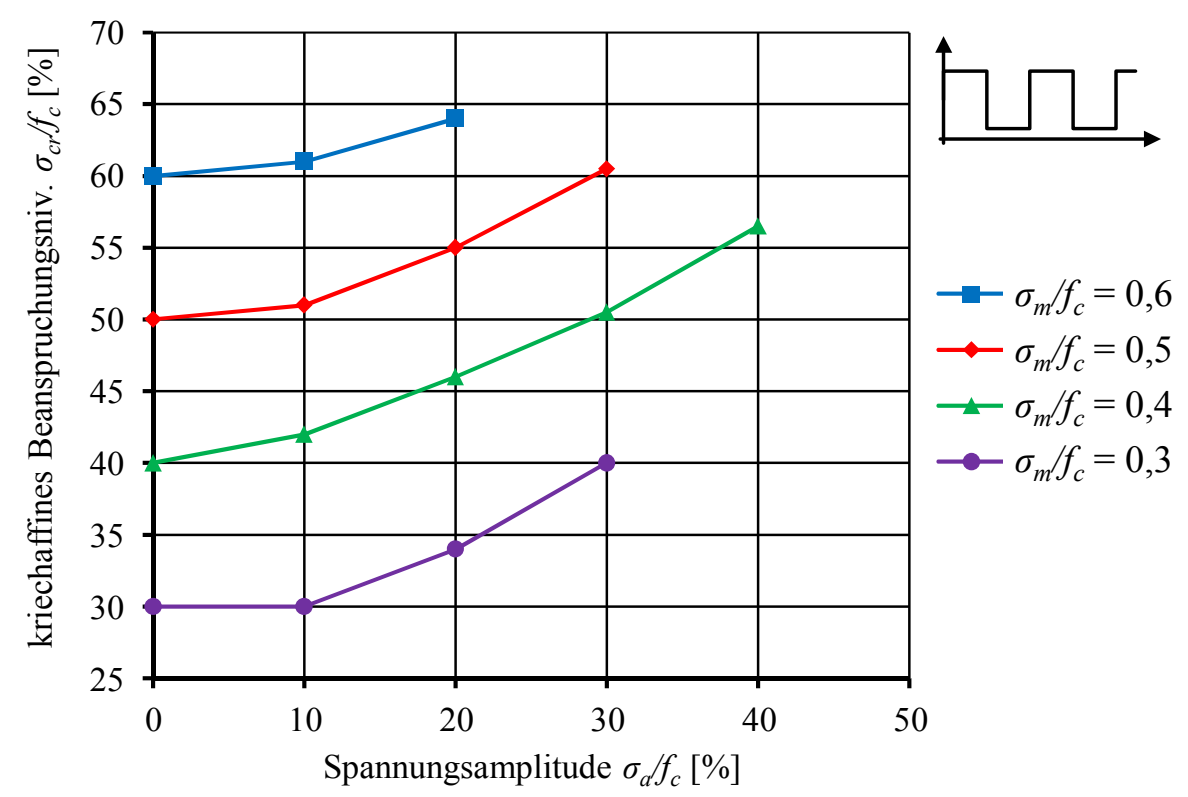

Abbildung 4.11: Kriechaffines Spannungsniveau in Abhängigkeit vom Mittelspannungsniveau und der Spannungsamplitude für einen rechteckförmigen, periodischen Beanspruchungsverlauf

Das kriechaffine Beanspruchungsniveau wurde für verschiedene Mittelspannungsniveaus und Spannungsamplituden eines rechteckförmigen, periodischen Beanspruchungsverlaufs mithilfe von Gleichung 4.16 bzw. 4.22 sowie Abbildung 4.9 bestimmt und in Abbildung 4.11 grafisch dargestellt. Untersucht wurden Oberspannungsniveaus bis $S_{o}=0,80$. Für eine Spannungsamplitude von null $\left(\sigma_{a}=0\right)$ entspricht der Ermüdungsversuch dem Kriechversuch. Für diesen Fall sind das Mittelspannungsniveau und das kriechaffine Spannungsniveau identisch. Mit 
zunehmender Spannungsamplitude nimmt das kriechaffine Spannungsniveau gegenüber dem Mittelspannungsniveau überproportional zu. Dies trifft jedoch nur dann zu, wenn das Oberspannungsniveau die angenommene Linearitätsgrenze des Betonkriechens übertrifft $\left(\sigma_{o}>0,4 \cdot f_{c}\right)$. Andernfalls entspricht das Mittelspannungsniveau dem kriechaffinen Spannungsniveau.

\subsection{Sinusförmiger Beanspruchungsverlauf}

Im folgenden Abschnitt wird das kriechaffine Beanspruchungsniveau für einen sinusförmigen Beanspruchungsverlauf bestimmt. In diesem Rahmen wird geprüft, ob das Ergebnis auch auf Grundlage der Erkenntnisse des vorhergehenden Abschnitts abgeleitet werden kann. Durch die kontinuierliche Änderung der Beanspruchung ist dies insbesondere für das reversible Modell, infolge des Rückkriechens des Betons, nicht unmittelbar zu beantworten. Auf beide Modelle werden sinusförmige Beanspruchungsverläufe aufgebracht. Die Belastungsfrequenzen von Ermüdungsversuchen liegen üblicherweise in einem Frequenzbereich von $0,1 \mathrm{~Hz}$ bis $10 \mathrm{~Hz}$. Entsprechend der vorhergehenden Ergebnisse dürfte die Belastungsfrequenz keinen Einfluss auf das kriechaffine Beanspruchungsniveau haben. Dennoch werden für die genannten Frequenzen die kriechaffinen Beanspruchungsniveaus separat ermittelt. Die untersuchten Frequenzen unterscheiden sich damit um den Faktor 100. In den Berechnungen werden das Mittelspannungsniveau und die Spannungsamplitude variiert. Die Bestimmung des kriechaffinen Belastungsniveaus erfolgt jeweils nach dem in Abbildung 4.2 dargestellten Schaubild bis zu einem Oberspannungsniveau von $S_{o}=0,80$.

Die Abbildung 4.12 stellt das kriechaffine Beanspruchungsniveau in Abhängigkeit vom Mittelspannungsniveau und der Spannungsamplitude dar. Gezeigt werden die Ergebnisse des irreversiblen Modells bei einer Belastungsfrequenz von $10 \mathrm{~Hz}$. Bei einer Amplitude von null entspricht das Mittelspannungsniveau definitionsgemäß dem kriechaffinen Spannungsniveau. Für den Fall, dass der Beanspruchungsverlauf den nichtlinearen Bereich des Kriechens durchfährt $\left(\sigma_{o}>0,4 \cdot f_{c}\right)$, nimmt das kriechaffine Spannungsniveau mit steigender Spannungsamplitude überproportional zu. Liegt das Oberspannungsniveau unterhalb dieser Grenze, entspricht das Mittelspannungsniveau unabhängig von der Amplitude dem kriechaffinen Spannungsniveau. Im Grundsatz gilt das Ergebnis aus Abbildung 4.11 analog für den sinusförmigen Beanspruchungsverlauf. Das kriechaffine Spannungsniveau ist bei einem sinusförmigen Beanspruchungsverlauf jedoch kleiner als bei einem rechteckförmigen Beanspruchungsverlauf bei gleicher Mittelspannung und Spannungsamplitude.

Entsprechend Abbildung 4.12 ergibt sich für ein Mittelspannungsniveau und eine Spannungsamplitude von $40 \%$ der Betondruckfestigkeit $\left(S_{o}=0,80 ; S_{u}=0\right)$ das kriechaffine Spannungsniveau zu etwa $50 \%$ der Betondruckfestigkeit. Die viskosen Verformungen unter der zyklischen Beanspruchung sind damit um den Faktor 1,45 größer als die Kriechverformungen auf dem Mittelspannungsniveau. Dieser Faktor ergibt sich aus dem höheren Spannungsverhältnis und 
dem spannungsabhängigen Faktor zur Berücksichtigung der überproportionalen Verformungen im Bereich des nichtlinearen Kriechens:

$$
\frac{\sigma_{c r}}{\sigma_{m}} \cdot \frac{k\left(\sigma_{c r}\right)}{k\left(\sigma_{m}\right)}=\frac{0,5 \cdot f_{c}}{0,4 \cdot f_{c}} \cdot \frac{k\left(0,5 \cdot f_{c}\right)}{k\left(0,5 \cdot f_{c}\right)}=\frac{0,5}{0,4} \cdot \frac{1,16}{1}=1,45
$$

Die kriechaffinen Beanspruchungsniveaus für eine Belastungsfrequenz von $0,01 \mathrm{~Hz}$ und die des irreversiblen Modells stimmen alle mit den Ergebnissen aus Abbildung 4.12 überein. Die Ergebnisse unterscheiden sich je Beanspruchungsniveau um weniger als $1 \%$. Diese Differenz ist auf Rechenungenauigkeiten zurückzuführen. Somit kann geschlossen werden, dass weder die Belastungsfrequenz noch das Rückkriechen des Betons einen Einfluss auf das kriechaffine Beanspruchungsniveau haben.

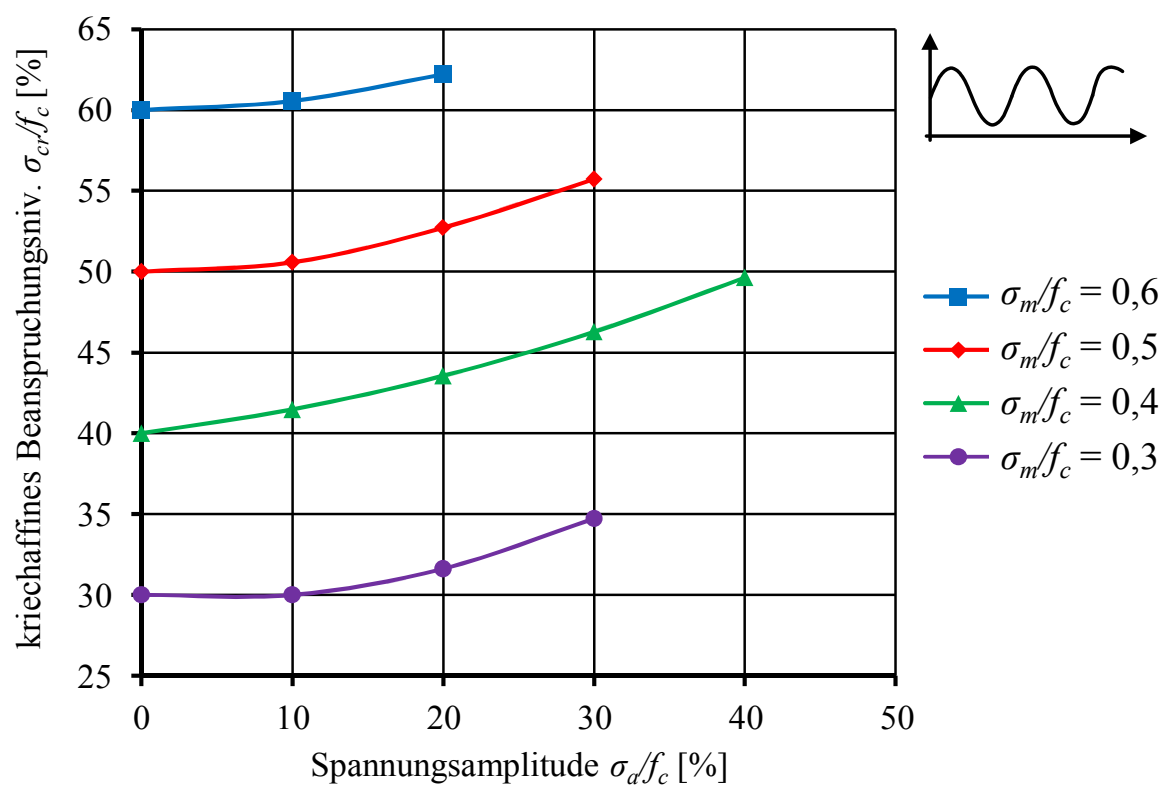
Abbildung 4.12: Kriechaffines Spannungsniveau in Abhängigkeit vom Mittelspannungsniveau
und der Spannungsamplitude für einen sinusförmigen Beanspruchungsverlauf

Die Kriechverformungen des kriechaffinen Beanspruchungsniveaus müssen definitionsgemäß den viskosen Verformungen des sinusförmigen Beanspruchungsverlaufs entsprechen. In Anlehnung an die Superpositionsmethode aus Abbildung 2.12 (rechts) kann dieser formale Zusammenhang durch die Gleichung 4.24 ausgedrückt werden.

$$
\sigma_{c r} \cdot\left[J\left(t_{0}\right)-J\left(t_{n}\right)\right] \cdot k\left(\sigma_{c r}\right)=\sum_{i=0}^{n} \sigma_{i}\left(t_{i}\right) \cdot\left[J\left(t_{i}\right)-J\left(t_{i+1}\right)\right] \cdot k\left(\sigma_{i}\right)
$$

Werden nun die auftretenden Verformungen innerhalb eines einzelnen Lastwechsels betrachtet, so kann angenommen werden, dass die Differenzen der Komplianzfunktion für die einzelnen Spannungsintervalle gleich groß sind. Damit können sie als Konstante aus der Summenfunktion gezogen werden und es gilt: 


$$
\begin{array}{r}
\sigma_{c r} \cdot \Delta J \cdot k\left(\sigma_{c r}\right)=\frac{\Delta J}{n} \cdot \sum_{i=0}^{n} \sigma_{i}\left(t_{i}\right) \cdot k\left(\sigma_{i}\right) \\
\Leftrightarrow \quad \sigma_{c r} \cdot k\left(\sigma_{c r}\right)=\frac{1}{n} \cdot \sum_{i=0}^{n} \sigma_{i}\left(t_{i}\right) \cdot k\left(\sigma_{i}\right)
\end{array}
$$

Die resultierende Gleichung stellt den formalen Zusammenhang zwischen dem kriechaffinen Beanspruchungsniveau und dem sinusförmigen Beanspruchungsverlauf dar. Sie ist unabhängig von der Komplianzfunktion bzw. dem Verlauf der Kriechfunktion und unabhängig von der Belastungsfrequenz. Zudem entspricht sie im Grundsatz der Gleichung 4.16 für einen periodischen, rechteckförmigen Verlauf.

Zur Veranschaulichung des formalen Zusammenhangs wird ein einzelner Lastwechsel eines sinusförmigen Beanspruchungsverlaufs betrachtet. Das Mittelspannungsniveau und die Spannungsamplitude werden wie zuvor zu $40 \%$ der Betondruckfestigkeit angenommen $\left(S_{o}=0,80 ; S_{u}=0\right)$. Der Lastwechsel wird für die Betrachtung in $n=10$ Lastschritte unterteilt. Damit ergibt sich auf Grundlage von Abbildung 4.13 das kriechaffine Beanspruchungsniveau zu:

$$
\begin{aligned}
\sigma_{c r} \cdot k\left(\sigma_{c r}\right)= & \frac{1}{10} \cdot\left[\sigma\left(t_{1}\right) \cdot k\left(\sigma_{1}\right)+\sigma\left(t_{2}\right) \cdot k\left(\sigma_{2}\right)+\ldots+\sigma\left(t_{10}\right) \cdot k\left(\sigma_{10}\right)\right] \\
\Leftrightarrow & \sigma_{c r} \cdot k\left(\sigma_{c r}\right)=\frac{1}{10} \cdot\left[\begin{array}{r}
2 \cdot 0,52 \cdot 1,20+2 \cdot 0,72 \cdot 1,62+1 \cdot 0,8 \cdot 1,82 \\
+2 \cdot 0,28 \cdot 1+2 \cdot 0,08 \cdot 1+1 \cdot 0 \cdot 1
\end{array}\right] \cdot f_{c} \\
\Leftrightarrow & \sigma_{c r} \cdot k\left(\sigma_{c r}\right)=0,577 \cdot f_{c}
\end{aligned}
$$

Wird nun unter Berücksichtigung von Abbildung 4.9 das kriechaffine Spannungsniveau abgelesen, ergibt sich dieses zu $\sigma_{c r} \approx 0,5 f_{c}$. Es entspricht damit dem Ergebnis aus Abbildung 4.12.

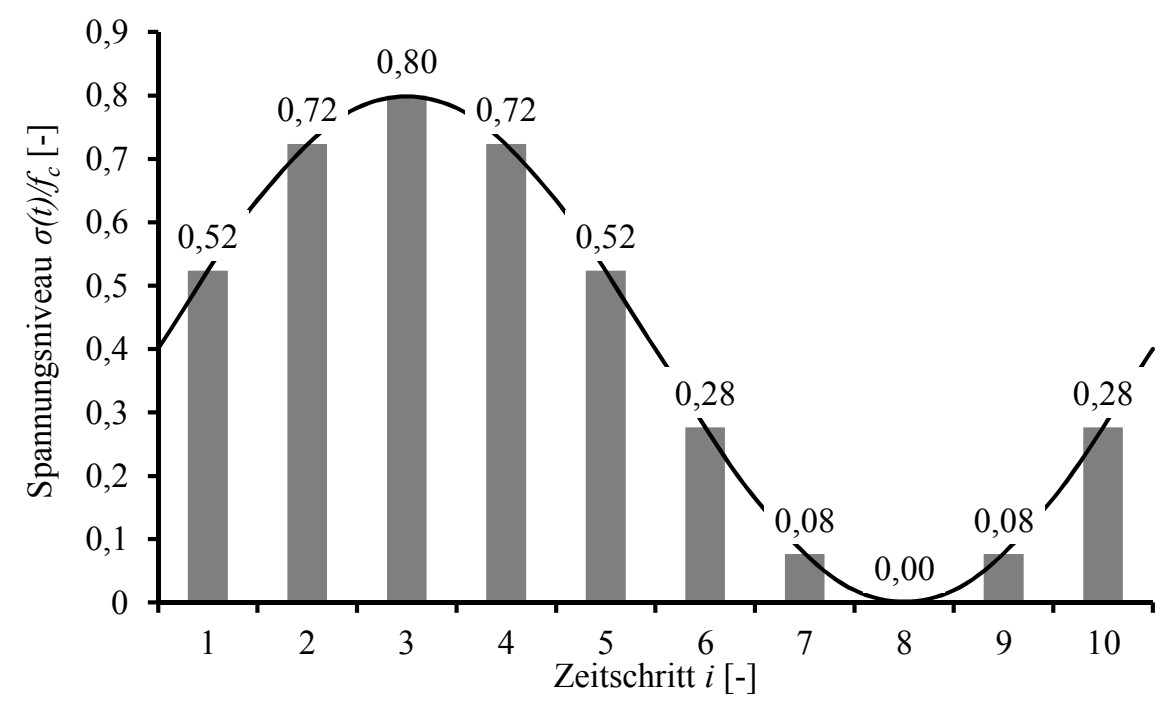

Abbildung 4.13: Betrachteter Lastwechsel zur Bestimmung des kriechaffinen Beanspruchungsniveaus

Die Abbildung 4.14 zeigt die Kriechkurven unter der zuvor genannten zyklischen Beanspruchung $\left(S_{o}=0,80 ; S_{u}=0\right)$ bei einer Belastungsfrequenz von $0,1 \mathrm{~Hz}$. Dargestellt sind die viskosen Verformungskurven des reversiblen und des irreversiblen Modells. Des Weiteren ist die 
Kriechkurve für das kriechaffine Beanspruchungsniveau von $50 \%$ der Betondruckfestigkeit dargestellt. Die Kurven sind deckungsgleich. Die Abweichungen zwischen den Verformungsverläufen sind kleiner als $1 \%$. Die geschlossene Ableitung des kriechaffinen Beanspruchungsniveaus, wie zuvor bei dem irreversiblen Modell, gelingt für das reversible Modell nicht. Die geringe Differenz besagt aber, dass bei den üblichen Belastungsfrequenzen im Ermüdungsversuch das kriechaffine Beanspruchungsniveau mithilfe des reversiblen oder dem irreversiblen Modell, und somit auch mithilfe der Gleichung 4.25, abgeschätzt werden kann.

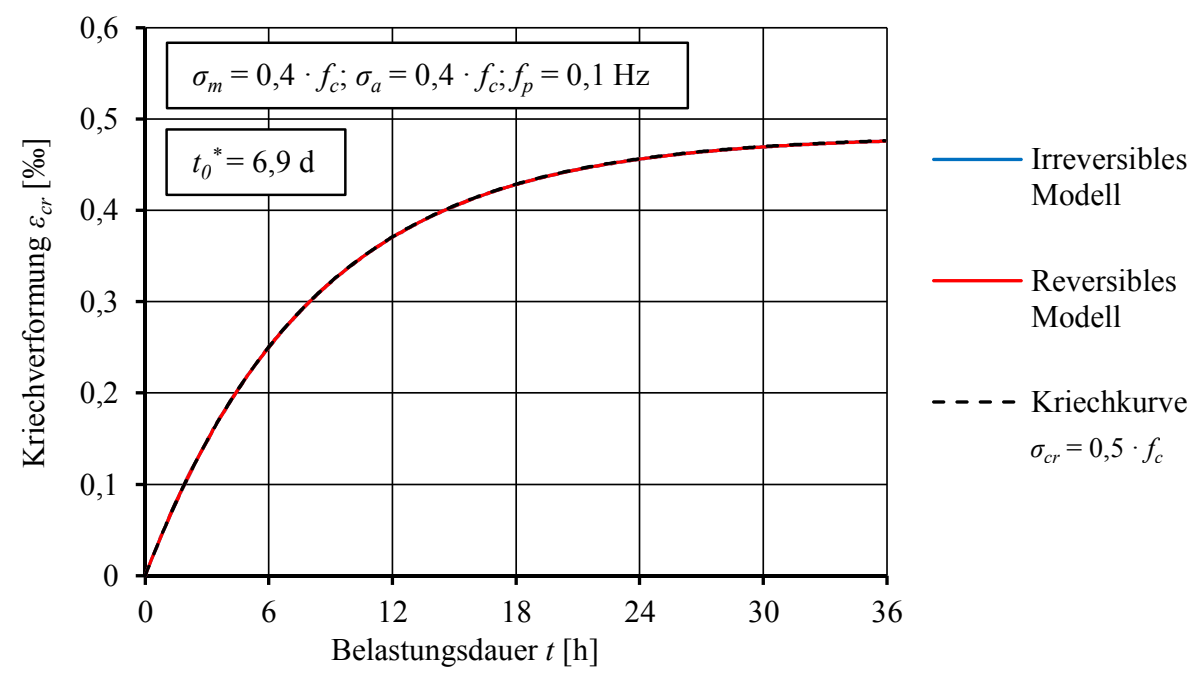

Abbildung 4.14: Viskoser Verformungsverlauf infolge einer zyklischen Beanspruchung bei einer Belastungsfrequenz von $f_{p}=0,1 \mathrm{~Hz}$

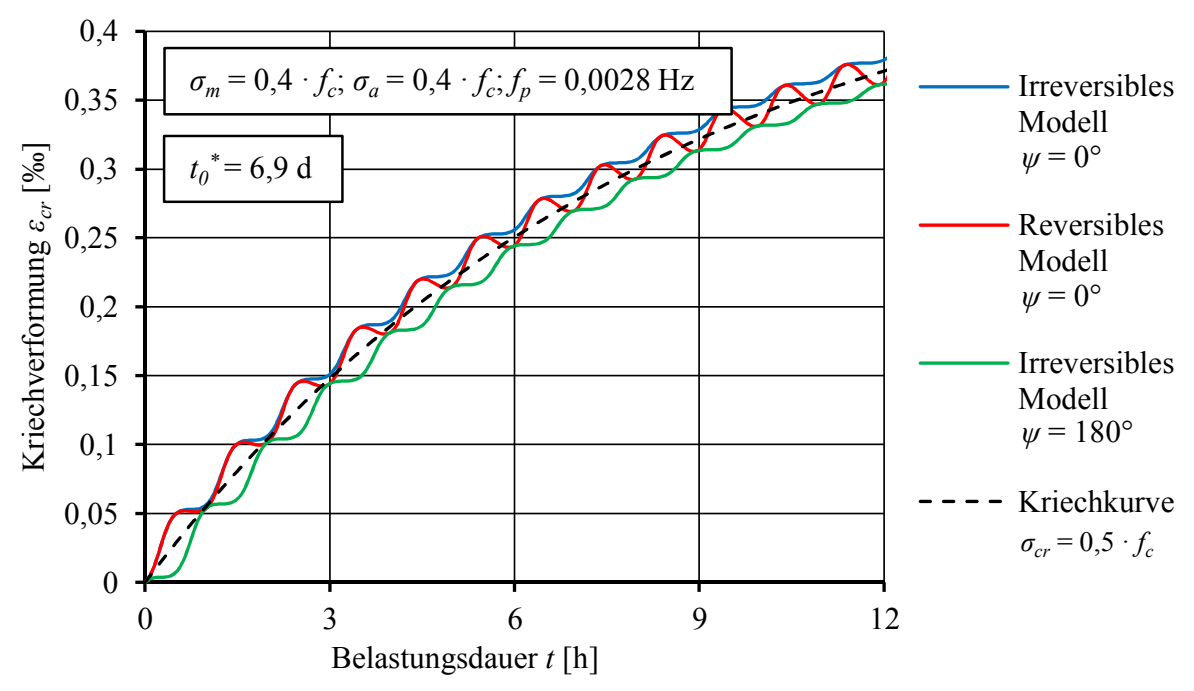

Abbildung 4.15: Viskoser Verformungsverlauf infolge einer zyklischen Beanspruchung bei einer Belastungsfrequenz von $f_{p}=0,0028 \mathrm{~Hz}$

Erst wenn die Belastungsfrequenz deutlich verringert wird, ist eine Differenzierung zwischen den Verformungsverläufen der beiden Modelle zu erkennen. Die Verformungsverläufe sind in der Abbildung 4.15 für eine Belastungsfrequenz 0,0028 Hz (1 Lastwechsel/Stunde) dargestellt. Das Oberspannungsniveau wurde wie zuvor $\mathrm{zu} 80 \%$ der Betondruckfestigkeit und das Unterspannungsniveau zu null gewählt. Aus dem Rückkriechen des Betons resultiert ein 
wellenförmiger Verformungsverlauf für das reversible Modell. Im Mittel entspricht der Verformungsverlauf aber weiterhin dem Kriechverlauf für das kriechaffine Beanspruchungsniveau von $\sigma_{c r}=0,5 f_{c}$. Der Verformungsverlauf des irreversiblen Modells mit einem Phasenwinkel von $\psi=0^{\circ}$ liegt oberhalb des dargestellten Kriechverlaufs. Wird der Phasenwinkel aber zu $\psi=180^{\circ}$ angenommen, liegt dieser unterhalb des Kriechverlaufs. An dieser Stelle wird also der Verlauf der Kriechfunktion zu Belastungsbeginn auf das Ergebnis des kriechaffinen Beanspruchungsniveaus überschätzt. Insgesamt sind die kriechaffinen Beanspruchungsniveaus für das reversible und das irreversible Modell im Mittel identisch und können anhand von Abbildung 4.12 bestimmt werden.

\subsection{Fazit}

Auf Grundlage der rheologischen Modellbildung und den numerischen Untersuchungen kann geschlussfolgert werden, dass die Belastungsfrequenz das kriechaffine Beanspruchungsniveau nicht beeinflusst. Es ist jedoch zu bedenken, dass die Belastungsgeschwindigkeit durch die impulsförmige Interpretation des zyklischen Beanspruchungsverlaufs in den vorgestellten Untersuchungen unberücksichtigt bleibt, vgl. Abbildung 4.3. Ebenso wird das kriechaffine Beanspruchungsniveau nicht von der Kriechfunktion beeinflusst. Somit ist lediglich der proportionale bzw. überproportionale Zusammenhang zwischen Kriechspannung und Kriechdehnung ausschlaggebend für das kriechaffine Beanspruchungsniveau. Die Abbildung 4.12 und 4.16 stellen das kriechaffine Beanspruchungsniveau in Abhängigkeit von der Mittelspannung und der Spannungsamplitude dar. Die Abbildungen gelten für den formalen Zusammenhang zwischen Kriechspannung und Kriechdehnung entsprechend Model Code 1990, Eurocode 2 und Model Code 2010. Bei abweichenden Verhältnissen kann das kriechaffine Spannungsniveau auf Grundlage von Gleichung 4.24 abgeschätzt werden.

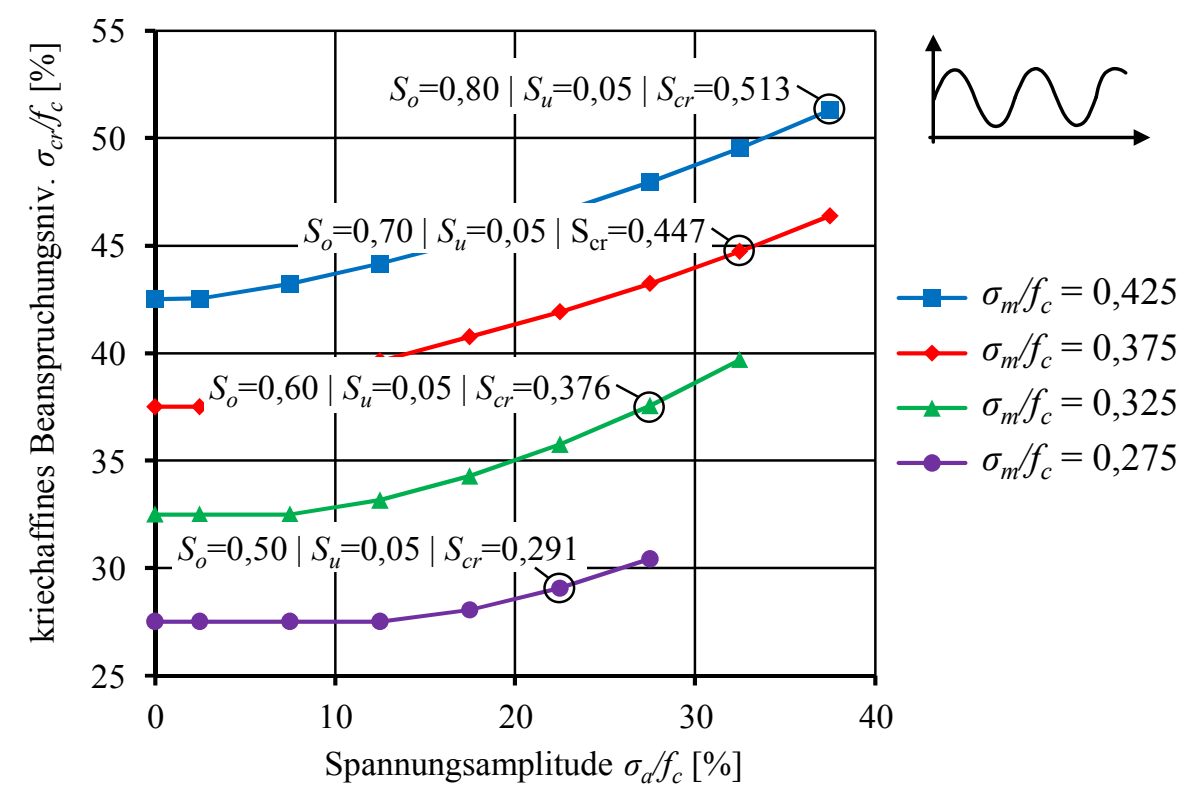

Abbildung 4.16: Kriechaffines Spannungsniveau in Abhängigkeit vom Mittelspannungsniveau und der Spannungsamplitude 
Ist das Oberspannungsniveau des zyklischen Beanspruchungsverlaufs kleiner als die Linearitätsgrenze des Betonkriechens, sind das kriechaffine Spannungsniveau und das Mittelspannungsniveau des zyklischen Beanspruchungsverlaufs identisch. Andernfalls liegt das kriechaffine Spannungsniveau zwischen dem Mittelspannungsniveau und dem Oberspannungsniveau. Die rechnerischen Untersuchungsergebnisse bestätigen somit die experimentellen Ergebnisse von (Whaley \& Neville, 1973), (Rostásy et al., 1975) und (Holmen, 1979).

Häufig werden Ermüdungsversuche bei einem Unterspannungsniveau von $5 \%$ der Betondruckfestigkeit und Oberspannungsniveaus von $80 \%, 70 \%, 60 \%$ und $50 \%$ der Betondruckfestigkeit durchgeführt. Für diese Spannungsniveaus ergeben sich Mittelspannungsniveaus, die von denen der Abbildung 4.12 abweichen. Die Abbildung 4.16 stellt in diesem Zusammenhang die kriechaffinen Beanspruchungsniveaus für diese sinusförmigen Beanspruchungsverläufe dar. 


\section{Experimentelle Untersuchungen ${ }^{1}$}

Die experimentellen Untersuchungen zur Verifizierung des kriechaffinen Beanspruchungsniveaus und der additiven Modellvorstellung werden nachfolgend präsentiert. Der Untersuchungsumfang stützt sich auf den in Abschnitt 3.3.2 geschilderten Untersuchungsbedarf. In diesem Zusammenhang werden die Betonzusammensetzung, die Versuchsdurchführung und Prüfmaschinentechnik sowie die Versuchsergebnisse dargestellt. Es handelt sich zunächst um eine allgemeine Darstellung der Versuchsergebnisse. Im anschließenden Kapitel 6 werden die Versuchsdaten hinsichtlich der auftretenden Dehnungsanteile gemäß der in Kapitel 3 vorgestellten additiven Modellvorstellung analysiert.

\subsection{Versuchsdurchführung}

\subsubsection{Betoneigenschaften und Probekörperherstellung}

Die Ermüdungs- und Kriechuntersuchungen erfolgten an zylindrischen, unbewehrten Betonproben. Der gesamte Probenumfang wurde aus einer Betoncharge hergestellt, um einen möglichen Einfluss unterschiedlicher Herstellungschargen ausschließen $\mathrm{zu}$ können. Die Betondruckfestigkeit sollte der Festigkeitsklasse C50/60 entsprechen. Die gewählte Betonfestigkeitsklasse entspricht damit einem höherfesten Normalbeton, wie sie heute bei dynamisch hochbeanspruchten Bauwerken eingesetzt werden. Die Frischbetonkonsistenz wurde $\mathrm{zu}$ „sehr weich“ (F4) gewählt, um eine gute Betonierbarkeit der zylindrischen Schalkörper zu gewährleisten. Nachfolgend ist die Betonzusammensetzung tabellarisch dargestellt.

Tabelle 5.1: Betonzusammensetzung

\begin{tabular}{|l|l|}
\hline w/z-Wert & 0,36 \\
\hline CEM I 42,5 R & $465 \mathrm{~kg} / \mathrm{m}^{3}$ \\
\hline Gesteinskörnung $0 / 16$ & $1.751 \mathrm{~kg} / \mathrm{m}^{3}$ \\
\hline Wassergehalt & $161 \mathrm{~kg} / \mathrm{m}^{3}$ \\
\hline PCE-Fließmittel & $6,0 \mathrm{~kg} / \mathrm{m}^{3}$ \\
\hline Verzögerer & $1,4 \mathrm{~kg} / \mathrm{m}^{3}$ \\
\hline
\end{tabular}

Die Schalungen bestanden aus handelsüblichen KG-Rohren des Typs DN 110, deren Enden zwischen Holzplatten verspannt wurden. Die Schalungen wurden mittels Schaufeln in zwei Lagen von oben befüllt und jeweils 30 Sekunden auf einem Rütteltisch verdichtet. Nach der Betonage wurden die Probekörper mit einer PE-Folie abgedeckt, um die auftretenden Feuchtigkeitsverluste zu reduzieren. Die zylinderförmigen Probekörper wurden nach 48 Stunden ausgeschalt und dann

\footnotetext{
${ }^{1}$ Auszüge des Kapitels wurden bereits in (von der Haar et al., 2015a), (von der Haar et al., 2015b) und (von der Haar \& Marx, 2016b) veröffentlicht.
} 
für mindestens 14 Tage bei einer Lufttemperatur von $20^{\circ} \mathrm{C}$ und $65 \%$ relativer Luftfeuchtigkeit gelagert, bevor mit der Probenvorbereitung begonnen wurde.

Im Rahmen der Probenvorbereitung wurden zunächst einige Zentimeter in Probenlängsrichtung der oberen und unteren Sektion der Betonzylinder abgesägt, um eventuell herstellungsbedingt gestörte Bereiche $\mathrm{zu}$ entfernen. Im nächsten Arbeitsschritt wurden die späteren Lasteinleitungsflächen planparallel geschliffen. Das Feinpolieren dieser Flächen komplettierte die Probenvorbereitung. Die Probenvorbereitung sollte eine möglichst zentrische Krafteinleitung und gleichmäßige Spannungsverteilung über den Probenquerschnitt sicherstellen.

Der Außendurchmesser der Probekörper betrug im Mittel 103 mm, die Probekörperlänge nach der Probenvorbereitung rund $280 \mathrm{~mm}$. Das Längen zu Durchmesser Verhältnis betrug damit in etwa 3/1, sodass davon auszugegangen werden kann, dass die Querdehnungen im mittleren Bereich der Probekörper nicht behindert werden.

Für die geplanten Kriechversuche wurden in Anlehnung an (DAfStb, 1991) Probekörper mit einer Länge von $600 \mathrm{~mm}$ hergestellt. Als Schalung wurden ebenfalls KG-Rohre des Typs DN 110 verwendet, wodurch sich der mittlere Durchmesser zu $103 \mathrm{~mm}$ ergab. Die Probekörper wurden genauso gelagert wie die zuvor beschriebenen, jedoch wurden diese maschinenbedingt weder planparallel geschliffen noch feinpoliert.

Zur Einordnung der Betondruckfestigkeitsklasse wurden Betonwürfel mit einer Kantenlänge von $150 \mathrm{~mm}$ hergestellt. Die mittlere Würfeldruckfestigkeit wurde nach 28 Tagen an drei bis zur Prüfung wassergelagerten Würfeln bestimmt. Die mittlere Würfeldruckfestigkeit ergab sich zu $f_{\text {cm,cube, } 28 d} \approx 65 \mathrm{~N} / \mathrm{mm}^{2}$. Der untersuchte Beton kann damit der angestrebten Festigkeitsklasse C50/60 zugeordnet werden. Die Festigkeitswerte der untersuchten Betonwürfel sind im Anhang A.1.1 aufgeführt. Die Untersuchung erfolgte nach (DIN EN 12390-3, 2009).

\subsubsection{Ermüdungsversuche}

Insgesamt wurden 30 Ermüdungsversuche mit den in Tabelle 5.2 dargestellten Beanspruchungsniveaus und Belastungsfrequenzen durchgeführt. Die aufgeführten Beanspruchungsgruppen resultieren aus Tabelle 3.1.

Tabelle 5.2: Beanspruchungskombinationen der durchgeführten Ermüdungsversuche

\begin{tabular}{|c|c|c|c|c|}
\hline \multirow{2}{*}{$\begin{array}{c}S_{o}=\sigma_{o} / f_{c} \\
{[-]}\end{array}$} & \multirow{2}{*}{$\begin{array}{c}S_{u}=\sigma_{u} / f_{c} \\
{[-]}\end{array}$} & \multirow{2}{*}{$\begin{array}{c}f_{p} \\
{[\mathrm{~Hz}]}\end{array}$} & \multicolumn{2}{|c|}{ Probekörperanzahl } \\
\hline & & & Serie I & Serie II \\
\hline 0,80 & \multirow{3}{*}{0,05} & 10 & 5 & - \\
\hline 0,70 & & 10 & 4 & 5 \\
\hline 0,60 & & 10 & 4 & 5 \\
\hline 0,80 & \multirow{3}{*}{0,05} & 1 & 3 & - \\
\hline 0,70 & & 1 & 2 & - \\
\hline 0,60 & & 1 & 2 & - \\
\hline
\end{tabular}


Die Ermüdungsversuche wurden zu zwei unterschiedlichen Zeitpunkten und in zwei unterschiedlichen Prüfmaschinen durchgeführt. Das Betonalter der Proben der Serie I betrug mindestens 44 Tage und das der Serie II mindestens 132 Tage bei Prüfbeginn. Das Probenalter bei Prüfbeginn der Serie II war damit 3-mal größer als das der Serie I, wodurch unterschiedliche Kriechdehnungen im Ermüdungsversuch zu erwarten waren. Die Versuche der Serie I wurden in einer 2,5 MN servohydraulischen Universalprüfmaschine und die Versuche der Serie II in einer $1,0 \mathrm{MN}$ servohydraulischen Universalprüfmaschine durchgeführt, vgl. Abbildung 5.1. Unmittelbar vor Beginn der jeweiligen Ermüdungsversuchsserie wurde die mittlere Betondruckfestigkeit $f_{c}$ an drei zylindrischen Betonproben in der jeweiligen Prüfmaschine bestimmt. Die Zylinderdruckfestigkeit diente der Bestimmung der jeweiligen bezogenen Beanspruchungsniveaus $\left(S_{o}, S_{u}\right)$ bzw. der Oberspannung $\sigma_{o}$ und der Unterspannung $\sigma_{u}$ im Ermüdungsversuch. Damit sollten Prüfmaschineneinflüsse ausgeschlossen und die Betonnacherhärtung berücksichtigt werden. Die Probekörper für die Druckfestigkeitsuntersuchungen entstammten der gleichen Betoncharge und wurden genauso gelagert und vorbereitet wie die Probekörper für die Ermüdungsversuche.

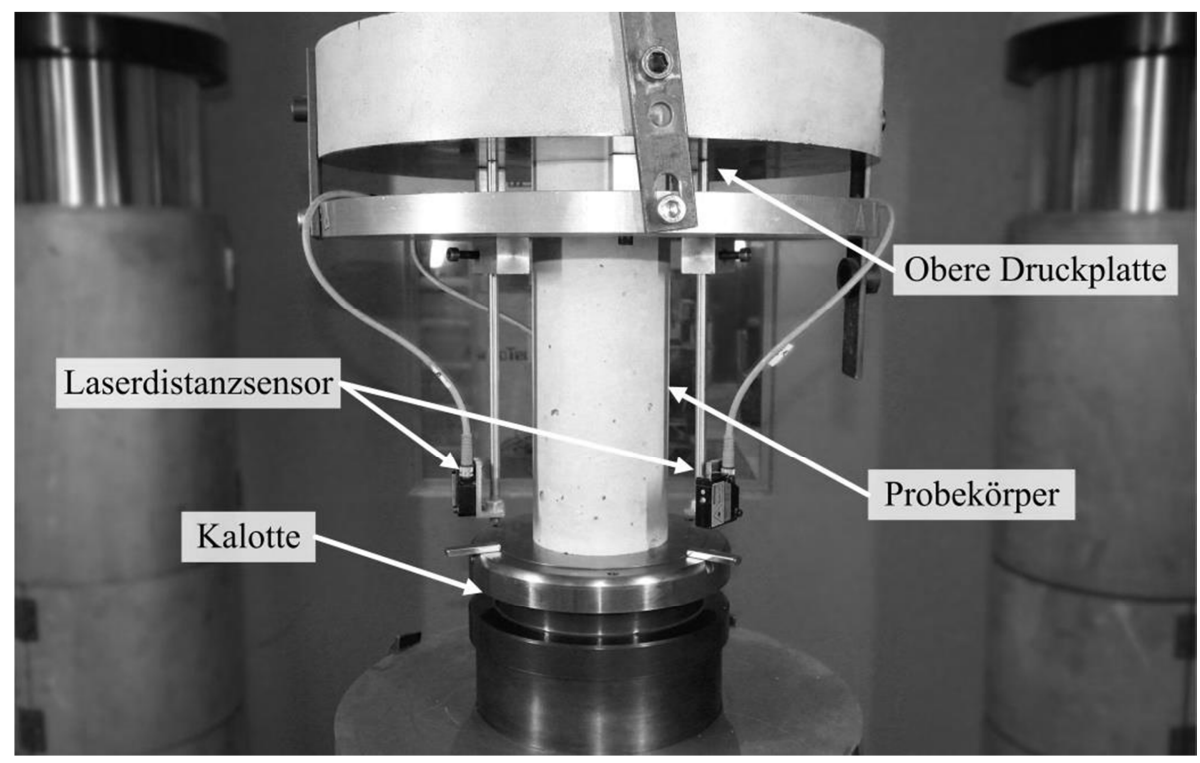

Abbildung 5.1: Prüf- und Messaufbau für die Ermüdungsversuche der Serie II

Nachdem der Probekörper zentrisch auf der unteren Druckplatte der Prüfmaschine bzw. der Kalotte ausgerichtet war, wurde der Kolben des Prüfzylinders bis kurz vor Erreichen des Kraftschlusses zwischen Probekörper und oberer Druckplatte ausgefahren. Zur Gewährleistung einer zentrischen Lasteinleitung, folgte optisch das parallele Ausrichten der oberen Prüffläche des Betonzylinders zur oberen Druckplatte der Prüfmaschine. Anschließend wurde der Kraftschluss zwischen Probekörper und Prüfmaschine hergestellt. Zur Bestimmung der Zylinderdruckfestigkeit wurde die Belastung kraftgeregelt mit einer konstanten Belastungsgeschwindigkeit von $0,5 \mathrm{~N} /\left(\mathrm{mm}^{2} \cdot \mathrm{s}\right)$ auf den Probekörper bis zum Bruch aufgebracht. Bei den Ermüdungsversuchen erfolgte nach dem Einrichten der Probe das kraftgeregelte Anfahren der Mittellast mit einer Belastungsgeschwindigkeit von $0,5 \mathrm{~N} /\left(\mathrm{mm}^{2} \cdot \mathrm{s}\right)$. Die zyklische Belastung wurde anschließend kraftgeregelt, sinusförmig und mit einer Belastungsfrequenz von $1 \mathrm{~Hz}$ bzw. $10 \mathrm{~Hz}$ aufgebracht. 
Das Durchläuferniveau wurde nicht wie üblich in Abhängigkeit einer definierten Bruchlastwechselzahl definiert, sondern die Versuchsdauer wurde auf maximal 3,5 Tage pro Versuch begrenzt. Bei einer Belastungsfrequenz von $1 \mathrm{~Hz}$ ergibt sich damit die maximal erreichbare Bruchlastwechselzahl zu 300.000 Lastwechseln und bei $10 \mathrm{~Hz}$ zu 3.000 .000 Lastwechseln. Bei dem geringsten Oberspannungsniveau von $60 \%$ der Betondruckfestigkeit und einer Belastungsfrequenz von $1 \mathrm{~Hz}$ ist auf diese Weise die zugehörige Wöhlerlinie nach Model Code 1990 deutlich überschritten. Im Hinblick auf die Versuchsdauer werden die $10 \mathrm{~Hz}$-Versuche im Vergleich zu den $1 \mathrm{~Hz}$-Versuchen durch dieses Abbruchkriterium nicht überdurchschnittlich früh abgebrochen. Gleichzeitig wird die Gesamtdauer der Untersuchungen auf ein vertretbares Maß begrenzt.

Während der Versuchsdurchführung wurden die aufgebrachte Kraft und der Weg des Prüfzylinders sowie die axialen Verformungen des Probekörpers bei einer Messfrequenz von $300 \mathrm{HZ}$ kontinuierlich gemessen und aufgezeichnet. Die axialen Verformungen erfassten drei Laserdistanzsensoren des Typs OWLG 4003 des Herstellers Welotec. Diese waren um 120 zueinander versetzt in Umfangsrichtung um den Probekörper herum angeordnet. Des Weiteren wurde die Oberflächentemperatur einiger Probekörper in Probenmitte und ca. $1 \mathrm{~cm}$ vom oberen und unteren Probenrand entfernt sowie die Lufttemperatur im Probenraum aufgezeichnet. Die Temperaturaufzeichnung erfolgte mit Thermoelementen des Typs K. Die Abbildung 5.1 zeigt den Prüfaufbau der Serie II. Die Temperatursensoren sind in der Abbildung nicht zu sehen.

\subsubsection{Kriechversuche}

Mit den Kriechversuchen wurde das viskose Verformungsverhalten des Betons erfasst. Es wurden drei Beanspruchungsniveaus, die bei $40 \%$ (2 Versuche), $50 \%$ (4 Versuche) und $60 \%$ (2 Versuche) der Zylinderdruckfestigkeit $f_{c}$ lagen, betrachtet. Die Versuche erfolgten in einem Prüfstand aufeinander folgend. Die Beanspruchungsdauer betrug im Regelfall zwei bis drei Wochen je Probekörper. Das Alter der Probekörper bei Belastungsbeginn $t_{0}$ ergab sich zu 61 bis 379 Tagen.

Die Abbildung 5.2 zeigt den verwendeten Kriechversuchsstand und dessen schematischen Aufbau. Ein Hydraulikzylinder erzeugt die auf den Betonzylinder einwirkende Druckkraft. Mit einer Handpumpe wird der hierzu erforderliche Öldruck aufgebaut. Während der Versuchsdurchführung nimmt der Öldruck wegen den zeitabhängigen Verformungen des Betons ab. Um diesem Druckabfall entgegenzuwirken, ist ein pneumatischer Blasenspeicher in dem Versuchsaufbau integriert. Gelegentliches Nachpumpen war erforderlich, um die Abweichungen zwischen dem Soll-Öldruck und dem Ist-Öldruck entsprechend (DafStb, 1991) auf $3 \% \mathrm{zu}$ begrenzen.

Während der Versuchsdurchführung wurden der Öldruck, zur Bestimmung der Prüfkraft, sowie die Probekörperverformungen kontinuierlich aufgezeichnet. Der Öldruck wurde von einem Druckaufnehmer des Typs P3TCP des Herstellers HBM aufgenommen. Die Probekörperverformungen registrierten 3 induktive Wegaufnehmer des Typs WA 10 des Herstellers HBM. 
Diese waren um $120^{\circ}$ zueinander versetzt in Umfangsrichtung um den Probekörper herum angeordnet. Die Messfrequenz betrug zu Versuchsbeginn $1 \mathrm{~Hz}$ und wurde mit zunehmender Versuchslaufzeit auf bis zu $0,003 \mathrm{~Hz}$ reduziert. Im Weiteren wurden die Umgebungstemperatur und die relative Luftfeuchte aufgezeichnet.
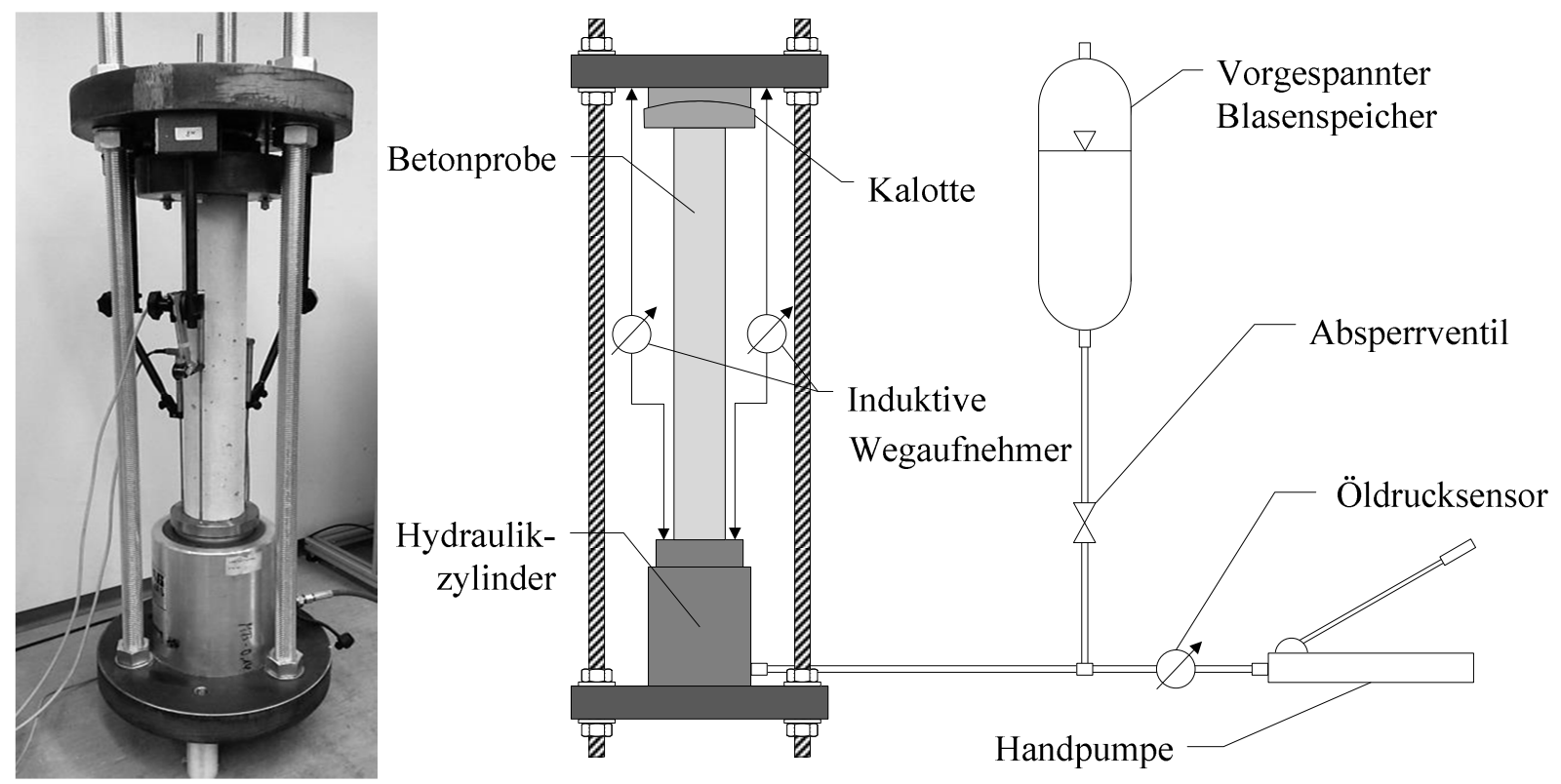

Abbildung 5.2: Prüf- und Messaufbau der Kriechversuche (links) und schematischer Versuchsaufbau (rechts)

Nach dem zentrischen Ausrichten des Probekörpers im Belastungsrahmen wurde der Kraftschluss zwischen Probekörper und Kalotte durch das Ausfahren des Hydraulikzylinders herbeigeführt. Anschließend wurde der Hydraulikdruck durch Betätigen der Handpumpe bis auf den Sollwert gesteigert. Dies gelang im Rahmen der Versuchsdurchführung innerhalb von 1 bis 2 Minuten entsprechend den Anforderungen nach (DAfStb, 1991). Direkt im Anschluss wurde das Absperrventil zum pneumatischen Blasenspeicher geöffnet, um die auftretenden Druckverluste infolge der zeitabhängigen Betonverformungen zu kompensieren.

\subsection{Darstellung der Versuchsergebnisse}

\subsubsection{Zylinderdruckfestigkeit}

Die mittlere Zylinderdruckfestigkeit, die unmittelbar vor Beginn der Ermüdungsversuche der Serien I und II jeweils an drei Zylindern bestimmt wurde, betrug $f_{c} \approx 70 \mathrm{~N} / \mathrm{mm}^{2}$. Abbildung 5.3 stellt die Spannungsdehnungsbeziehungen der untersuchten Betonproben grafisch dar. Trotz des unterschiedlichen Probenalters zwischen den Versuchsserien sind die erreichten Festigkeiten nahezu gleich groß.

Die Bruchdehnungen des untersuchten Betons, hier definiert als Dehnung bei Erreichen der maximalen Spannung, lagen bei der Serie I im Bereich von -3,59\% bis -3,72\%o und bei der Serie II im Bereich von $-3,30 \%$ bis $-3,49 \%$. Die mittlere Bruchdehnung betrug $\varepsilon_{c}=-3,53 \%$. Die Ergebnisse können dem Anhang A.1.2 im Detail entnommen werden. 
Die Spannungsdehnungslinien verlaufen bis zu einem Beanspruchungsniveau von $70 \%$ der Betondruckfestigkeit $\left(\sigma_{c} \approx 50 \mathrm{~N} / \mathrm{mm}^{2}\right)$ nahezu linear. Erst im anschließenden Bereich nimmt die Krümmung infolge von Rissbildungs- und plastischen Verformungsprozessen (vgl. Abschnitt 2.1) bis zum Versagen überproportional zu.

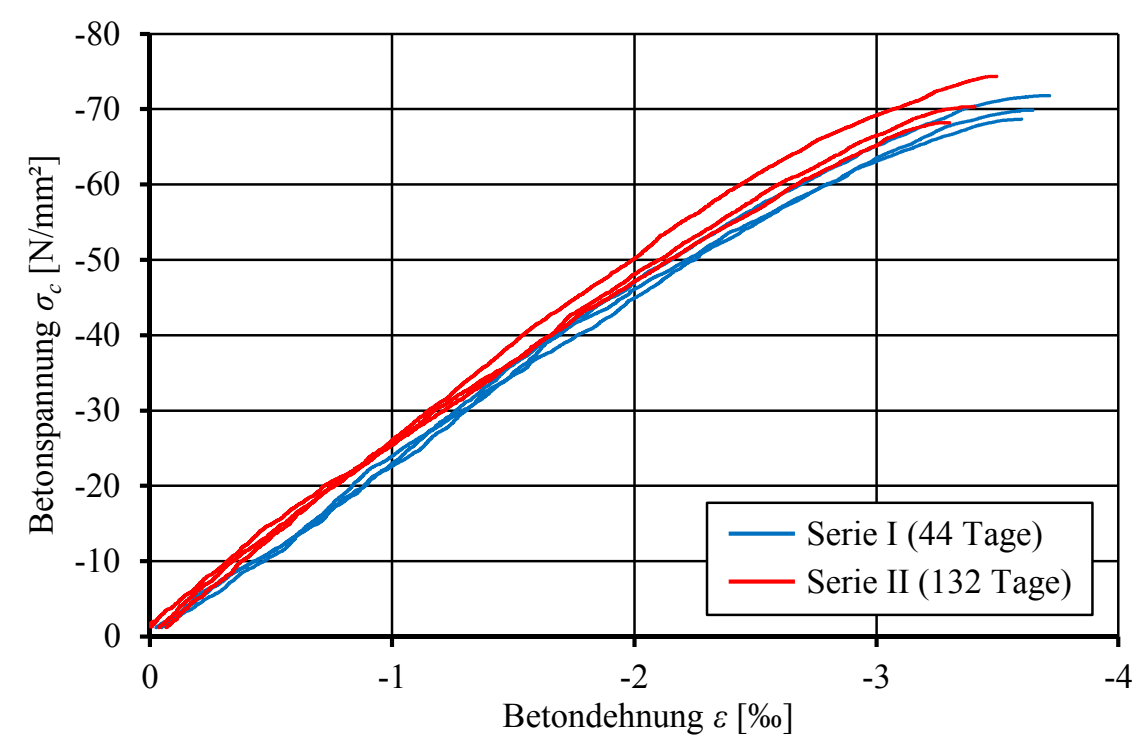

Abbildung 5.3: Spannungsdehnungsbeziehung des untersuchten Betons

\subsubsection{Bruchlastwechselzahlen}

Die Bruchlastwechselzahlen der Ermüdungsversuche und die Mittelwerte der Bruchlastwechselzahlen je Beanspruchungsgruppe werden in Abbildung 5.4 sowie Tabelle 5.3 dargestellt. Im Anhang A.2 sind die Einzelergebnisse im Detail aufgelistet. Die Wöhlerkurven des Model Code 1990 und des Model Code 2010 für ein Unterspannungsniveau von $S_{u}=0,05$ sind in der Abbildung 5.4 ergänzend enthalten.

Die erreichten Bruchlastwechselzahlen liegen im Bereich der Wöhlerlinien des Model Code 1990 und des Model Code 2010. Dabei weisen die Versuchsergebnisse, unabhängig von der Belastungsfrequenz und der Versuchsserie, bei einer bezogenen Oberspannung von $S_{o}=0,80$ und 0,60 eine deutlich größere Streubreite auf als die Bruchlastwechselzahlen bei $S_{o}=0,70$. Wie Abbildung 5.4 zeigt, liegen bei $S_{o}=0,80$ die mittleren Bruchlastwechselzahlen der $10 \mathrm{~Hz}$ - und der $1 \mathrm{~Hz}$-Versuche über dem Erwartungswert des Model Code 2010. Bei dem Beanspruchungsniveau von $S_{o}=0,70$ liegen die $1 \mathrm{~Hz}-$ Versuch oberhalb der zugehörigen Wöhlerlinie des Model Code 2010, wohingegen die $10 \mathrm{~Hz}$-Versuche unterhalb dieser liegen. Bei dem Oberspannungsniveau von $S_{o}=0,60$ erreicht lediglich ein $10 \mathrm{~Hz}$-Versuch die Wöhlerlinie nach Model Code 2010 . Ansonsten liegen die Bruchlastwechselzahlen tendenziell im Bereich der Wöhlerlinie des Model Code 1990. Die dargestellten $1 \mathrm{~Hz}$-Versuche des Beanspruchungsniveaus wurden bei $\log N=5,5$ $(\approx 300.000$ Lastwechsel) gemäß dem zuvor genannten Abbruchkriterium beendet. Die Proben wiesen zu diesem Zeitpunkt keine Anzeichen für ein nahes Versagen auf. Der 
Ermüdungswiderstand dieser Durchläuferversuche ist unbekannt, er ist aber höher als die dargestellten Werte.

Der Probekörper, der bei dem Oberspannungsniveau von $S_{o}=0,60$ die Wöhlerlinie des Model Code 2010 erreichte, wurde als Ausreißer eingestuft, vgl. Abbildung 5.4. Der Probekörper versagte nach ca. 2 Mio. Lastwechseln. Die Bruchlastwechselzahl des Probekörpers ist damit 3mal größer als die mittlere Bruchlastwechselzahl der zugehörigen Beanspruchungsgruppe inklusive dem Ausreißer. Auf Grundlage des Gubbs-Tests und des Residuen-Tests kann dieser Probekörper ebenfalls als Ausreißer gewertet werden (Fischer, 1999). Dementsprechend wurde der Probekörper im Rahmen der folgenden Datenauswertung nicht berücksichtigt. Die in Abbildung 5.4 und Tabelle 5.3 dargestellten mittleren Bruchlastwechselzahlen wurden ohne Berücksichtigung der Bruchlastwechselzahl des Ausreißers bestimmt.

Werden die Bruchlastwechselzahlen der Versuchsserie I isoliert betrachtet, erreichen die $10 \mathrm{~Hz}-$ Versuche auf dem Oberspannungsniveau von $S_{o}=0,80$ im Mittel höhere Bruchlastwechselzahlen als die zugehörigen $1 \mathrm{~Hz}-$ Versuche. Dieses Ergebnis deckt sich mit den Versuchsergebnissen in (Holmen, 1979), (Schneider et al., 2012) und (Oneschkow, 2014). Diese zeigen, dass höhere Belastungsfrequenzen auch zu höheren Bruchlastwechselzahlen führen. Für die niedrigeren Spannungsniveaus kehrt sich dieser Trend um. Die Versuche mit der höheren Belastungsfrequenz weisen niedrigere Bruchlastwechselzahlen auf. Diese Versuchskörper erwärmen sich während der Versuchsdurchführung deutlich stärker als die Versuchskörper mit der niedrigeren Belastungsfrequenz. In (von der Haar et al., 2015a) und (Elsmeier, 2015) wird die Reduktion der Bruchlastwechselzahl mit der Probekörpererwärmung in Zusammenhang gebracht, vgl. auch Abschnitt 5.2.3.

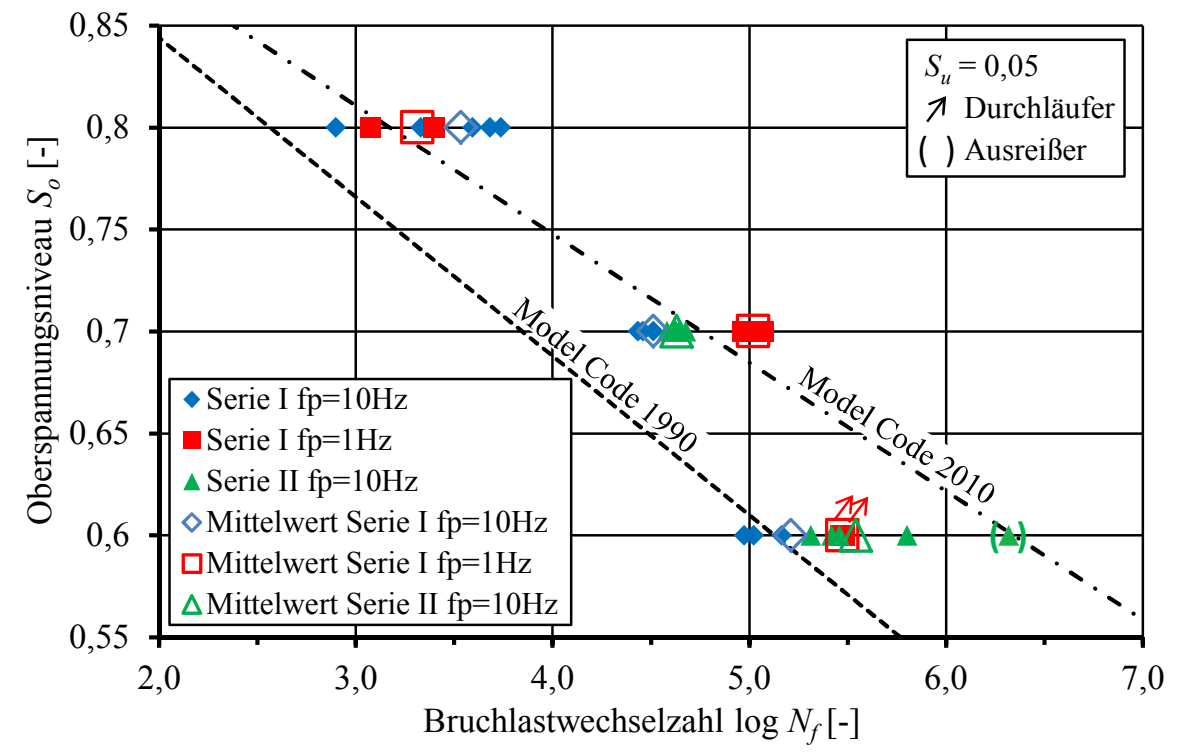

Abbildung 5.4: Bruchlastwechselzahlen der Ermüdungsversuche

Die $10 \mathrm{~Hz}$-Versuche der Serie II weisen im Mittel höhere Bruchlastwechselzahlen auf als die $10 \mathrm{~Hz}$-Versuche der Serie I. Die Beanspruchungsniveaus wurden jeweils auf den zugehörigen 
Wert der Betondruckfestigkeit unter monoton steigender Beanspruchung bezogen. Damit kann der Einfluss einer möglichen Betonnacherhärtung und ein möglicher Prüfmaschineneinfluss ausgeschlossen werden. Eine Ursache für dieses Verhalten ist derzeit nicht bekannt, unter Umständen wird es durch die abnehmende Probekörperfeuchte mit zunehmendem Betonalter hervorgerufen.

Tabelle 5.3: Mittlere Bruchlastwechselzahlen der Ermüdungsversuche

\begin{tabular}{|c|c|c|c|c|c|c|}
\hline Serie & $\begin{array}{l}S_{o} \\
{[-]}\end{array}$ & $\begin{array}{l}S_{u} \\
{[-]}\end{array}$ & $\begin{array}{c}f_{p} \\
{[\mathrm{~Hz}]}\end{array}$ & $\begin{array}{l}N_{f} \\
{[-]}\end{array}$ & $\begin{array}{c}\log N_{f} \\
{[-]}\end{array}$ & $\begin{array}{c}\text { Anzahl } \\
{[-]}\end{array}$ \\
\hline \multirow{3}{*}{ I } & 0,80 & \multirow{3}{*}{0,05} & \multirow{3}{*}{10} & 3.419 & 3,53 & 5 \\
\hline & 0,70 & & & 32.598 & 4,51 & 4 \\
\hline & 0,60 & & & 163.163 & 5,21 & 4 \\
\hline \multirow{3}{*}{ I } & 0,80 & \multirow{3}{*}{0,05} & \multirow{3}{*}{1} & 2.060 & 3,31 & 3 \\
\hline & 0,70 & & & 105.136 & 5,02 & 2 \\
\hline & 0,60 & & & $298.350^{*}$ & 5,47 & 2 \\
\hline \multirow{2}{*}{ II } & 0,70 & \multirow{2}{*}{0,05} & \multirow{2}{*}{10} & 42.866 & 4,63 & 5 \\
\hline & 0,60 & & & $348.470^{* *}$ & 5,54 & $4(5)$ \\
\hline $\begin{array}{l}\text { Durchlä } \\
\text { Ausreiß }\end{array}$ & enth & & & & & \\
\hline
\end{tabular}

\subsubsection{Temperaturentwicklung}

Die Probekörper erwärmen sich im Ermüdungsversuch in Abhängigkeit von der Spannungsamplitude, der Belastungsfrequenz und der Versuchsdauer unterschiedlich stark. Die aufgezeichneten Temperaturverläufe sind im Anhang A.2.2 im Detail dargestellt. Die Abbildung 5.5 stellt die Änderungen der Oberflächentemperatur auf halber Probenhöhe für ausgewählte Versuchskörper in Abhängigkeit von der Lastwechselzahl dar. Bei einer bezogenen Oberspannung von $S_{o}=0,70$ führte eine Belastungsfrequenz von $10 \mathrm{~Hz}$ zu einer zwischen $20 \mathrm{~K}$ bis $25 \mathrm{~K}$ höheren Oberflächentemperatur als bei einer Belastungsfrequenz von $1 \mathrm{~Hz}$. Bei einer bezogenen Oberspannung von $S_{o}=0,60$ ist diese Differenz noch größer. Die $10 \mathrm{~Hz}-\mathrm{Versuche}$ erwärmten sich um bis zu $47 \mathrm{~K}$, wohingegen bei den $1 \mathrm{~Hz}$-Versuchen nur eine Erhöhung von ca. $6 \mathrm{~K}$ registriert wurde. Bei dem dargestellten $1 \mathrm{~Hz}$-Versuch ist darauf hinzuweisen, dass es sich um einen Durchläuferversuch handelt, der nach dem Erreichen von 290.575 Lastwechseln ohne Versagen der Probe abgebrochen wurde. Bei einem Oberspannungsniveau von $S_{o}=0,80$ sind nur geringe Temperaturänderungen zu erkennen. Die Temperaturerhöhung des $10 \mathrm{~Hz}-$ Versuchs betrug ca. $6 \mathrm{~K}$ und die des $1 \mathrm{~Hz}$-Versuchs ca. $4 \mathrm{~K}$. Der Ermüdungsversuch, der auf dem Oberspannungsniveau von $S_{o}=0,70$ bei einer Belastungsfrequenz von $1 \mathrm{~Hz}$ durchgeführt wurde, weist im Bereich von 60.000 bis 70.000 Lastwechseln einen Temperaturabfall auf. Dieser ist auf eine Änderung der Umgebungstemperatur zurückzuführen.

Wird die Probekörpererwärmung in Abhängigkeit von der Versuchsdauer betrachtet, so ist die Steigung der Temperaturverläufe und dementsprechend die Wärmefreisetzungsrate pro Zeiteinheit bei den $10 \mathrm{~Hz}$-Versuchen wesentlich größer als bei den $1 \mathrm{~Hz}-$ Versuchen, vgl. Abbildung 5.6. Des Weiteren nimmt die Steigung mit zunehmender Spannungsamplitude zu. Die 
Wärmefreisetzungsrate der Probekörper pro Zeiteinheit ist damit insbesondere von der Belastungsfrequenz und der Spannungsamplitude abhängig, die Gesamttemperaturänderung zusätzlich von der Versuchsdauer.

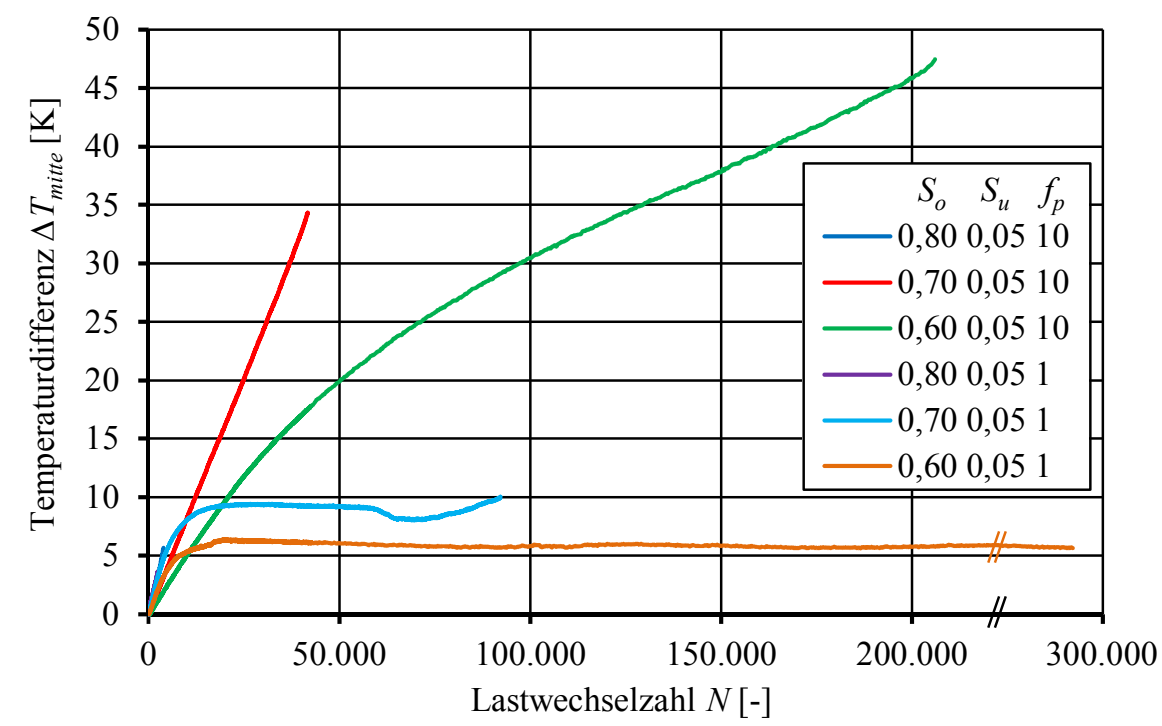

Abbildung 5.5: Probekörpererwärmung im Ermüdungsversuch auf halber Probenhöhe in Abhängigkeit von der Lastwechselzahl

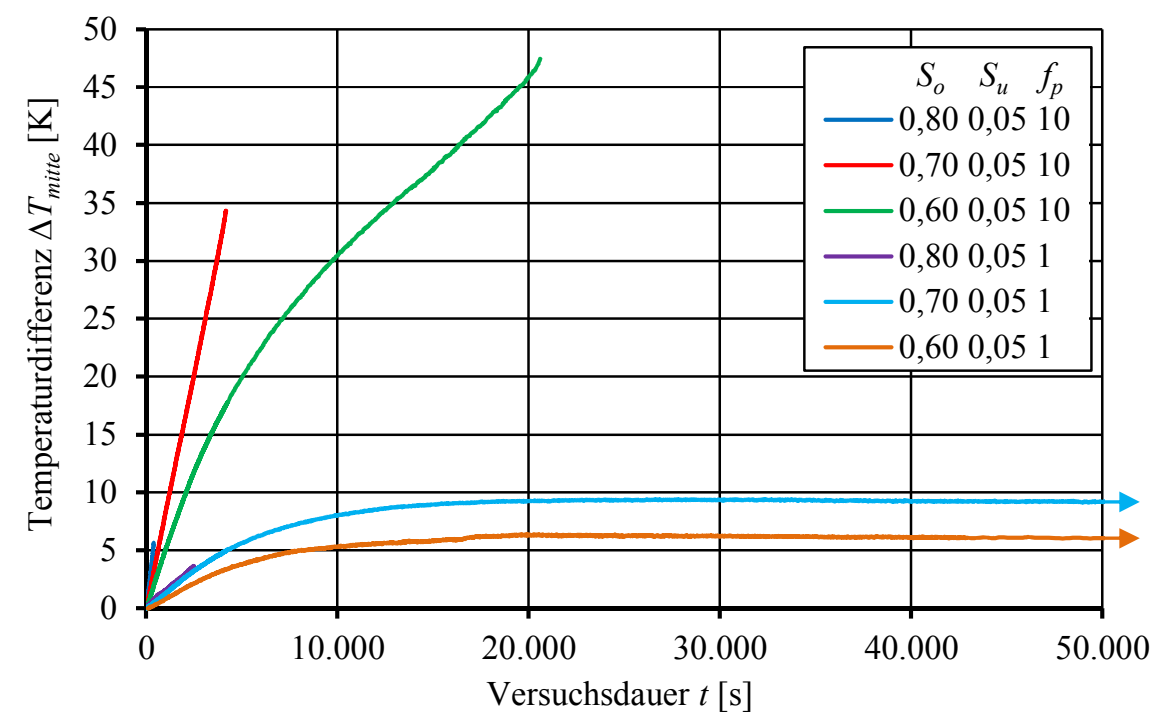

Abbildung 5.6: Probekörpererwärmung im Ermüdungsversuch auf halber Probenhöhe in Abhängigkeit von der Versuchsdauer

\subsubsection{Steifigkeitsentwicklung}

Aus den axialen Verformungsmessungen an den Betonproben wurde der Sekantenmodul $E_{s}$ entsprechend Gleichung 2.29 für sämtliche Lastwechsel bestimmt. Diese wurden in den folgenden Darstellungen immer auf den Sekantenmodul des ersten Entlastungsastes $E_{s}{ }^{0}$ bezogen. Im Anhang A.2.3 werden die Steifigkeitsverläufe aller Versuchskörper dargestellt. Bei den $10 \mathrm{~Hz}-$ Versuchen streuen die Verläufe wesentlich stärker als bei den $1 \mathrm{~Hz}$-Versuchen. In den Grafiken sind des Weiteren die gemittelten Steifigkeitsverläufe der einzelnen Beanspruchungsgruppen abgebildet. 
In der Anfangsphase bis ca. $10 \%$ der bezogenen Bruchlastwechselzahl nimmt die Steifigkeit der Probekörper zunächst sehr schnell ab. Im folgenden Bereich nimmt die Steifigkeit nur langsam $\mathrm{ab}$, während sich die Steifigkeitsabnahme in der letzten Phase wieder beschleunigt, bis die Probekörper versagen.

Die Abbildung 5.7 stellt die gemittelten und bezogenen Steifigkeitsverläufe der $10 \mathrm{~Hz}-$ Versuche vergleichend gegenüber. Niedrigere Oberspannungsniveaus weisen eine ausgeprägtere Steifigkeitsdegradation auf als höhere Oberspannungsniveaus. So nimmt der Sekantenmodul bei den Versuchen des niedrigsten Oberspannungsniveaus $\left(S_{o}=0,60\right)$ im Mittel auf $66 \%$ der Anfangssteifigkeit ab, während die Steifigkeit für die Versuche des höchsten Oberspannungsniveaus $\left(S_{o}=0,80\right)$ auf etwa $79 \%$ abnimmt. Gegenüber den Darstellungen von (Holmen, 1979) und (Do et al., 1993) ist die Gesamtsteifigkeitsänderung also auch vom Oberspannungsniveau abhängig. Das Gefälle der Steifigkeitsverläufe nimmt in der Abbildung 5.7 mit abnehmendem Oberspannungsniveau zu. Werden die Steifigkeitsverläufe aber in Abhängigkeit von der Lastwechselzahl dargestellt, so ist die Steifigkeitsänderung pro Lastwechsel bei den niedrigeren Oberspannungsniveaus geringer als bei den höheren.

Die bezogene Gesamtsteifigkeitsänderung der Ermüdungsprobekörper ist vom Oberspannungsniveau abhängig. Gleichzeitig beeinflusst das Oberspannungsniveau die Bruchlastwechselzahl der Probekörper maßgeblich. Aufgrund dessen zeigt Abbildung 5.8 die bezogene Steifigkeit unmittelbar vor dem Bruch der Probekörper in Abhängigkeit von der Bruchlastwechselzahl. Die Darstellung lässt eine lineare Abhängigkeit zwischen der bezogenen Sekantensteifigkeit und der logarithmierten Bruchlastwechselzahl erkennen. Die Standardabweichung der Versuchsergebnisse liegt in der Größenordnung von $\pm 4,3 \%$. Die Streuungen nehmen mit sinkendem Oberspannungsniveau zu.

Die Abbildung 5.9 stellt die berechneten Sekantenmoduln des ersten Entlastungsastes $E_{s}{ }^{0}$ und des Entlastungsastes unmittelbar vor dem Versagen der Betonkörper $E_{s}{ }^{B}$ dar. Bei Versuchsbeginn nimmt der Sekantenmodul mit abnehmendem Oberspannungsniveau tendenziell zu. Dies ist auf eine reduzierte Rissbildung bei geringeren Druckspannungsniveaus zurückzuführen. Demgegenüber nimmt die verbleibende Steifigkeit der Betonkörper unmittelbar vor dem Versagen mit dem Oberspannungsniveau ab. Somit ist nicht nur die bezogene Steifigkeit, sondern auch die tatsächliche Reststeifigkeit der Probekörper bei niedrigeren Oberspannungsniveaus geringer als bei höheren Oberspannungsniveaus. Die makroskopische Steifigkeitsdegradation der Betonkörper wird durch Rissbildungsprozesse initiiert. Die Steifigkeitsdegradation ist somit ein Indiz für die Gefügezerstörung im Betonkörper. Eine größere Steifigkeitsdegradation deutet auf eine stärkere Gefügezerstörung hin. Im Umkehrschluss bedeutet dies, dass niedrigere Oberspannungsniveaus bis zu einem höheren Grad der Gefügeschädigung aufgenommen werden können. Dementsprechend versagen ermüdungsbeanspruchte Betonproben vornehmlich während der Belastungsphase des zyklischen Beanspruchungsverlaufs unmittelbar vor dem Erreichen des Oberspannungsniveaus, vgl. Abbildung 5.11. Ein höherer Grad der Gefügeschädigung lässt aber auch größere schädigungsinduzierte Dehnungen erwarten. Diese These ist im Rahmen von Kapitel 6 zu prüfen. 


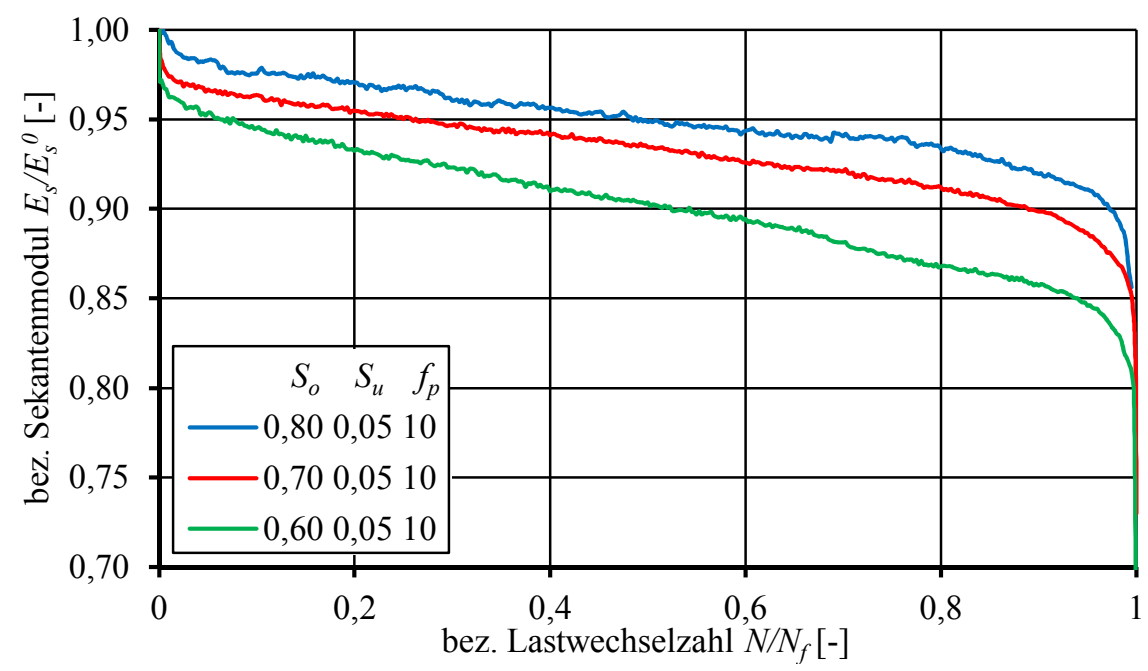

Abbildung 5.7: Entwicklung des Sekantenmoduls in Abhängigkeit von dem Beanspruchungsniveau

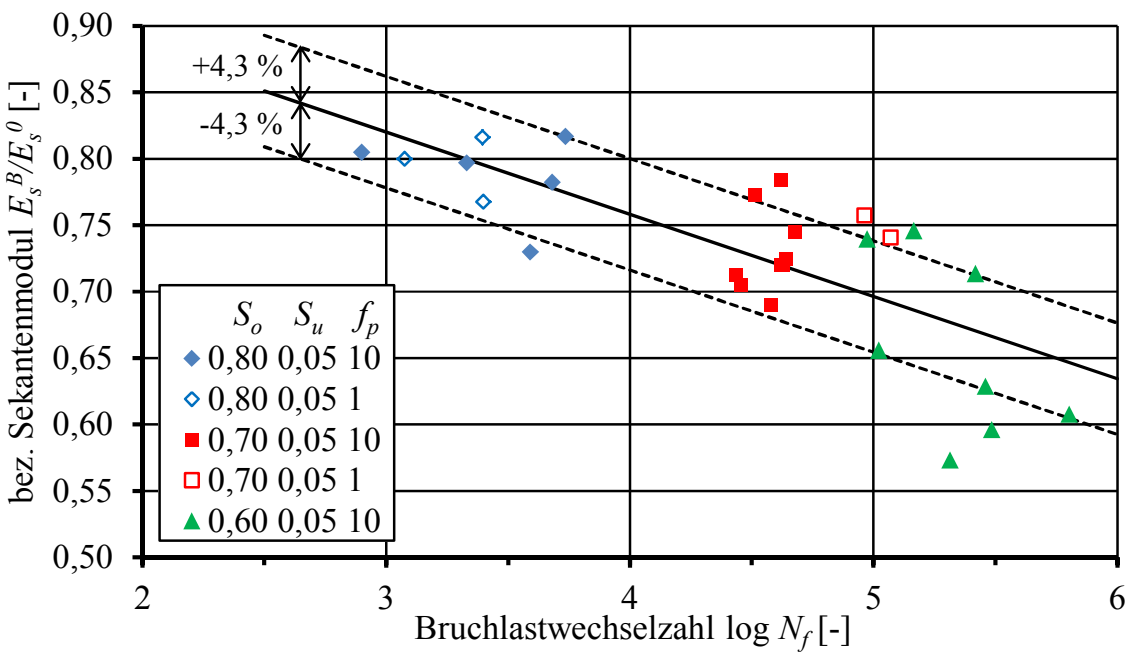

Abbildung 5.8: Bezogener Sekantenmodul in Abhängigkeit von der Bruchlastwechselzahl

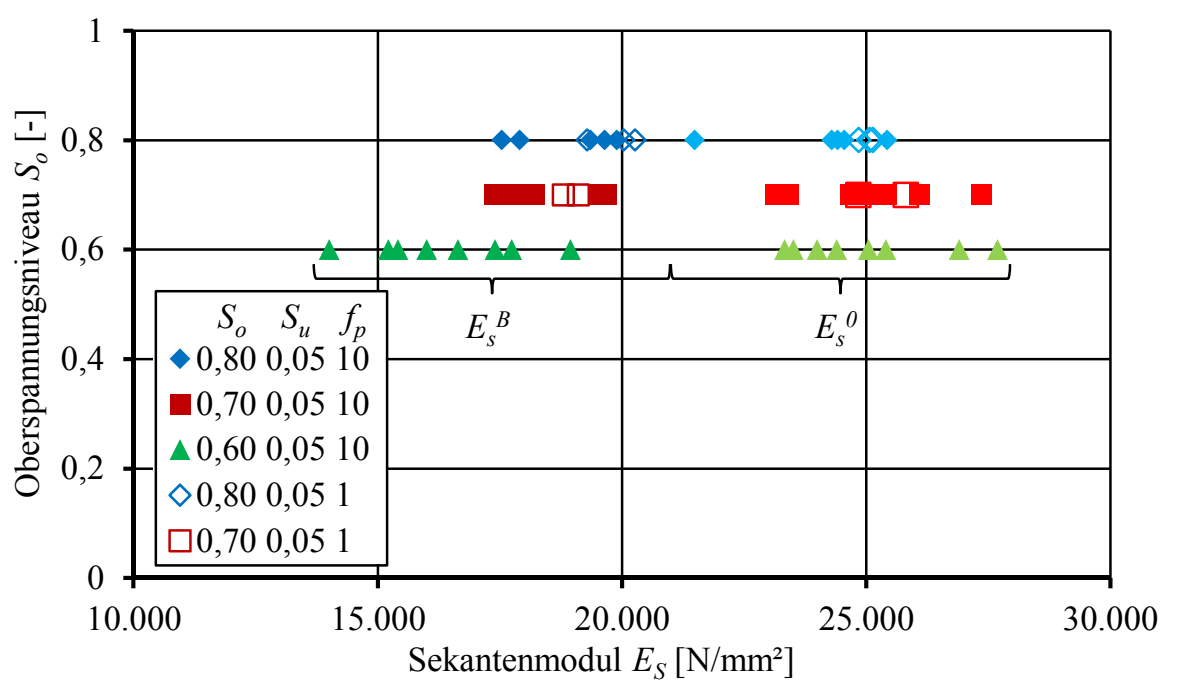

Abbildung 5.9: Sekantenmodul des ersten und letzten Entlastungsastes 


\subsubsection{Dehnungsverhalten}

Nachfolgend wird das Verformungsverhalten der Betonproben im Ermüdungsversuch betrachtet. Die Dehnungsverläufe der Probekörper auf dem Ober- und Unterspannungsniveau sind im Anhang A.2.4 dargestellt. Die Verläufe stellen sich als dreiphasige Dehnungsevolution dar. Der Phasenübergang von Phase I zu Phase II wird in der Literatur gewöhnlich bei $10 \%$ bis $20 \%$ und der Phasenübergang von Phase II zu Phase III bei $80 \%$ bis $90 \%$ der ertragbaren Bruchlastwechselzahl angegeben. Die erste und dritte Phase ist bei normalfesten Betonen in der Regel etwas länger und ausgeprägter als bei hochfesten und ultrahochfesten Betonen. (Holmen, 1979) untersuchte einen Normalbeton $\left(f_{c m, c u b e, 28 d}=40 \mathrm{~N} / \mathrm{mm}^{2}\right)$ und ermittelte die Phasenübergänge zu $n^{I / I I}=10 \%$ bzw. $n^{I I / I I I}=80 \%$. (Oneschkow, 2014) bestimmte die Phasenübergänge für den von ihr untersuchten hochfesten Beton $\left(f_{c m, c u b e, 28 d}=116 \mathrm{~N} / \mathrm{mm}^{2}\right)$ grafisch $\mathrm{zu} n^{I / I I}=11 \%$ bzw. $n^{I I / I I I}=93 \%$, während sich die Phasenübergänge des von (Wefer, 2010) untersuchten ultrahochfesten Betons $\left(f_{\text {cm, cube, } 28 d}=160 \mathrm{~N} / \mathrm{mm}^{2}\right)$ bei $n^{I / I I}=5 \%$ bzw. $n^{I I / I I I}=95 \%$ darstellten. Die Phasenübergänge des hier untersuchten höherfesten Normalbetons $\left(f_{c m, c u b e, 28 d}=65 \mathrm{~N} / \mathrm{mm}^{2}\right)$ wurden grafisch bestimmt und ergaben sich im Mittel zu $n^{I / I I}=9 \%$ bzw. $n^{I I / I I I}=92 \%$. Die dritte Phase der Dehnungsentwicklung ist damit verhältnismäßig kurz. Im Grundsatz stimmen die Phasenübergänge aber mit den in der Literatur angegebenen Werten überein.

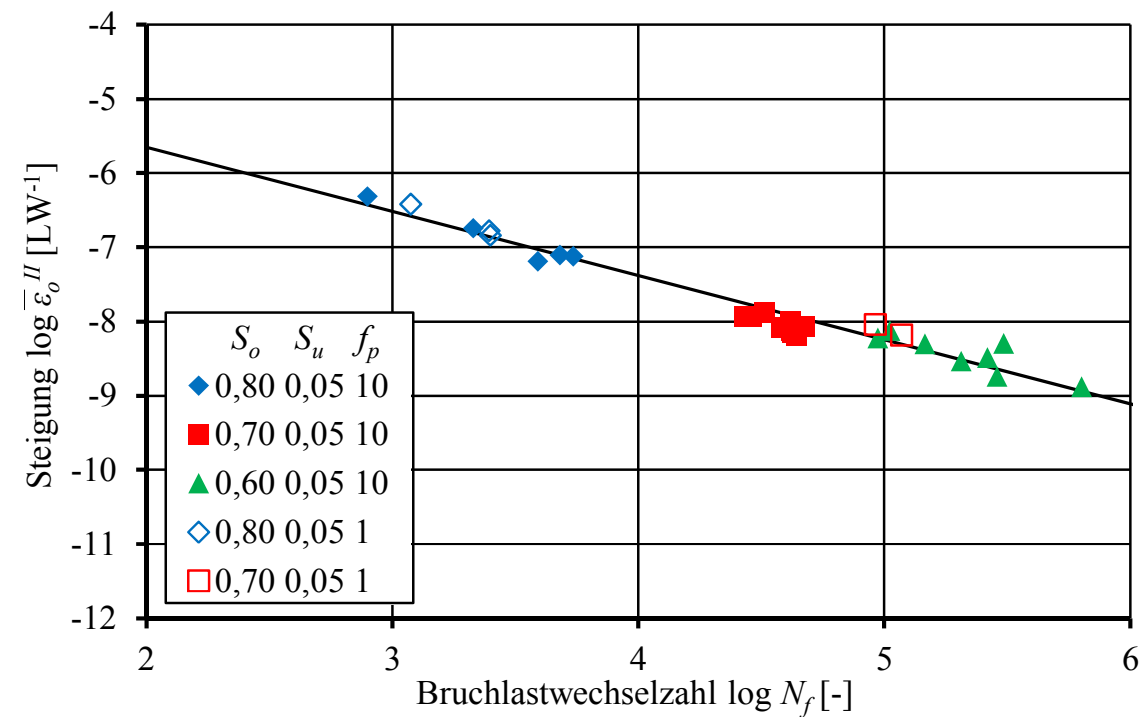

Abbildung 5.10: Steigung in der zweiten Phase auf dem Oberspannungsniveau

Die Steigungen der Dehnungsverläufe in der zweiten Phase auf dem Oberspannungsniveau $\varepsilon_{o}^{-I I}$ und dem Unterspannungsniveau $\bar{\varepsilon}_{u}^{-I I}$ wurde für alle Ermüdungsversuche ausgewertet. In der Abbildung 5.10 sind die Steigungen auf dem Oberspannungsniveau gegenüber den Bruchlastwechselzahlen $N_{f}$ grafisch aufgetragen. Im doppelt logarithmischen Maßstab ergibt sich ein linearer Zusammenhang zwischen beiden Größen. Die zugehörigen Ausgleichsgeraden für die Ober- und Unterspannungsniveaus sind in den Gleichungen 5.1 und 5.2 dargestellt und stellen unter Betrachtung der Bestimmtheitsmaße $R^{2}$ von 0,964 bzw. 0,927 einen guten linearen Zusammenhang trotz der unterschiedlichen Versuchsrandbedingungen (Beanspruchungsniveau und Belastungsfrequenz) dar. Die Gleichungen ergeben, dass die Steigungen der 
Dehnungsverläufe auf dem Oberspannungsniveau größer sind als auf dem Unterspannungsniveau. Dies ist auf die abnehmende Probekörpersteifigkeit während der Versuchsdurchführung zurückzuführen.

$$
\begin{array}{ll}
\log \bar{\varepsilon}_{o}^{I I}=-0,865 \cdot \log N_{f}-3,919 & R^{2}=0,964 \\
\log \bar{\varepsilon}_{u}^{I I}=-0,817 \cdot \log N_{f}-4,280 & R^{2}=0,927
\end{array}
$$

Die Gesamtverformungen bis zum Bruch der Betonkörper werden über die Ermüdungsbruchdehnung beschrieben. Als Ermüdungsbruchdehnung wird der Dehnungswert aufgefasst, der im letzten Lastwechsel auf dem Oberspannungsniveau $\varepsilon_{o}{ }^{B}$ bzw. dem Unterspannungsniveau $\varepsilon_{u}{ }^{B}$ erreicht wird. Als Ermüdungsbruchdehnung wird damit nicht der Dehnungswert bezeichnet der zum Zeitpunkt des Probekörperversagens auftritt, vgl. Abbildung 5.11 .
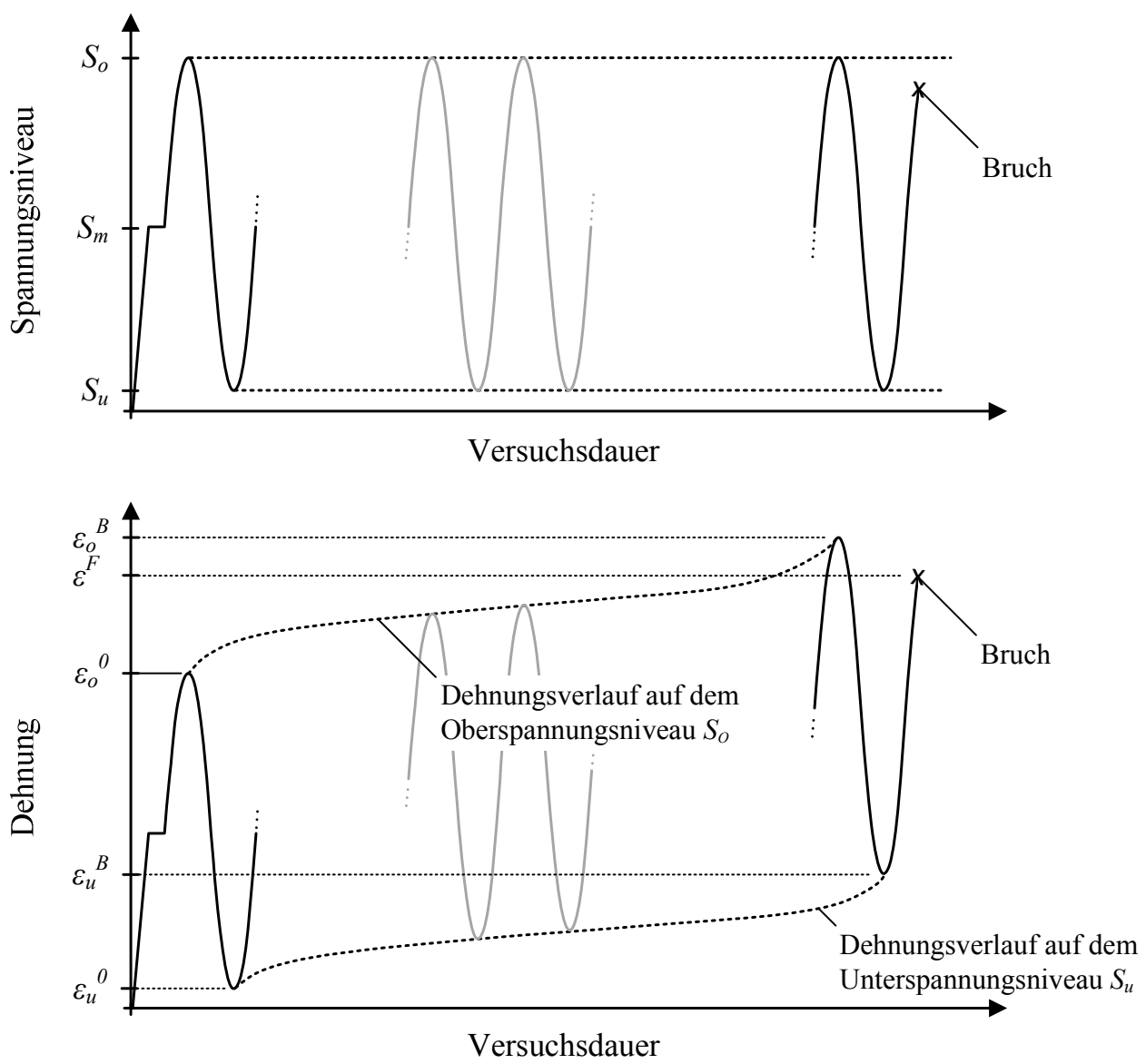

Abbildung 5.11: Schematische Definition der Ermüdungsbruchdehnung

In Abbildung 5.12 wird die Ermüdungsbruchdehnung $\varepsilon_{o}{ }^{B}$ der Ermüdungsversuche in Abhängigkeit von dem Oberspannungsniveau dargestellt. In dem Diagramm ist zusätzlich der Bereich der Bruchdehnungen unter monoton steigender Beanspruchung grau hinterlegt, vgl. Abschnitt 5.2.1. Auffallend ist, dass bei einem Großteil der Versuche die Ermüdungsbruchdehnungen in den Bereich der Bruchdehnungen fallen. Die Ermüdungsbruchdehnungen der 
übrigen Versuchskörper liegen sowohl unterhalb als auch oberhalb dieses Bereiches in einem Wertebereich von $-2,97 \%$ bis $-4,31 \%$. Der Mittelwert der Ermüdungsbruchdehnung liegt bei $\varepsilon_{o}{ }^{B}=-3,52 \%$ und stimmt mit der mittleren Bruchdehnung unter monoton steigender Beanspruchung von $\varepsilon_{c}=-3,53 \%$ nahezu exakt überein.

Für das Oberspannungsniveau von $S_{o}=0,70$ fällt die deutliche Abhängigkeit der Ermüdungsbruchdehnung von der Belastungsfrequenz auf. Die Versuche, die bei einer Belastungsfrequenz von $10 \mathrm{~Hz}$ durchgeführt wurden, weisen eine mittlere Ermüdungsbruchdehnung von -3,41\%o (Serie I) bzw. -3,27\%o (Serie II) auf, wohingegen die Versuche, die bei einer Belastungsfrequenz von $1 \mathrm{~Hz}$ durchgeführt wurden, eine mittlere Ermüdungsbruchdehnung von $-4,22 \%$ aufweisen. Auf Grundlage der in Kapitel 3 und 4 vorgestellten Modellvorstellung steht die resultierende, mittlere Dehnungsdifferenz von $0,88 \%$ im Zusammenhang mit den auftretenden thermischen und viskosen Verformungen während der Versuchsdurchführung. Die Temperaturdifferenz zwischen diesen beiden Versuchsreihen lag in der Größenordnung von $20 \mathrm{~K}$ bis $25 \mathrm{~K}$ (Oberflächentemperatur auf halber Probenhöhe) und die Versuchsdauer der $10 \mathrm{~Hz}$-Versuche betrug ca. 1 Stunde wohingegen die $1 \mathrm{~Hz}-$ Versuche etwa 30 Stunden dauerten.

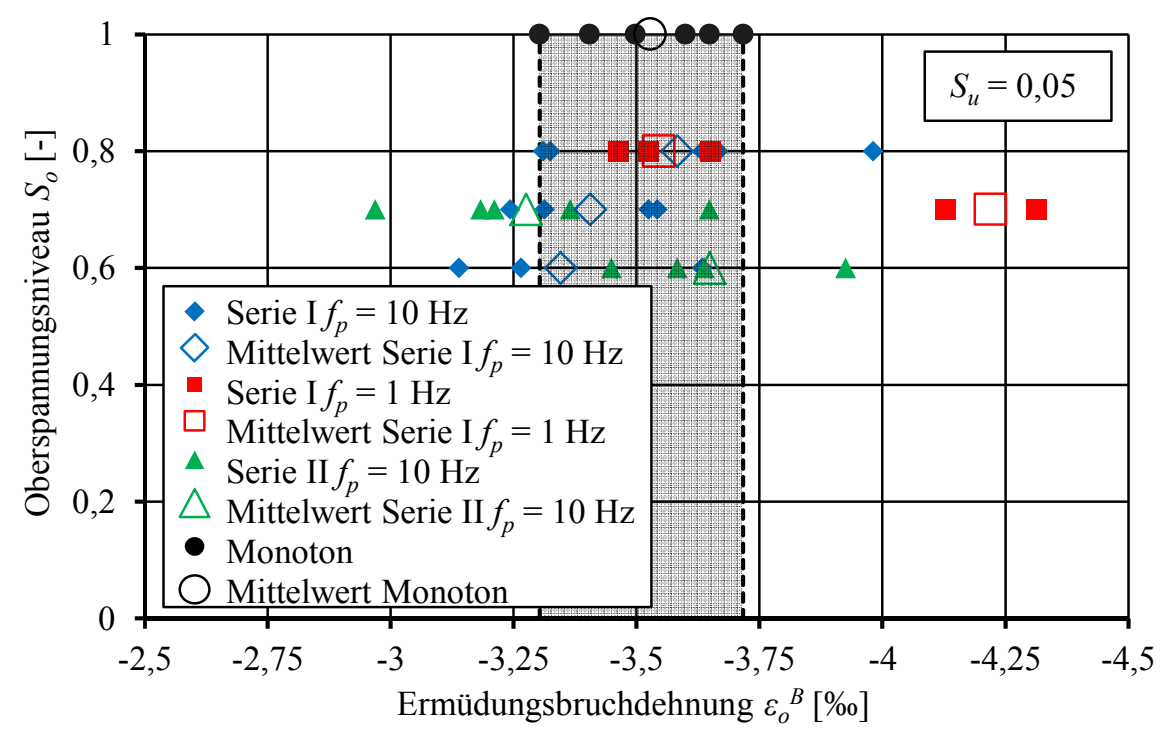

Abbildung 5.12: Ermüdungsbruchdehnungen auf dem Oberspannungsniveau in Abhängigkeit vom Oberspannungsniveau

Für das Oberspannungsniveau von $S_{o}=0,70$ ergeben sich für die $10 \mathrm{~Hz}$-Versuche der Serie II kleinere Ermüdungsbruchdehnungen als für die der Serie I. Die Dehnungsdifferenz beträgt 0,14 \%. Die Proben der Serie II wiesen bei Versuchsbeginn ein größeres Betonalter auf als die Proben der Serie I. Es bleibt zu prüfen, ob die Dehnungsdifferenz aus der reduzierten Kriechfähigkeit des Betons resultiert.

Auf dem Oberspannungsniveau von $S_{o}=0,80$ liegen die mittleren Ermüdungsbruchdehnungen der $1 \mathrm{~Hz}$ - und der $10 \mathrm{~Hz}$-Versuche sehr eng zusammen. Die Ursache wird in der relativ ähnlichen Probekörpererwärmung und in der verhältnismäßig kurzen Versuchsdauer gesehen. Hierdurch 
treten weder signifikante Temperaturdehnungen noch viskose Verformungen während der Versuchsdurchführung auf. Auffällig sind jedoch die starken Streuungen der $10 \mathrm{~Hz}$-Versuche auf dem genannten Beanspruchungsniveau $\left(S_{o}=0,80\right)$.

Auf dem geringsten Oberspannungsniveau von $S_{o}=0,60$ liegen nur Ermüdungsbruchdehnungen für die $10 \mathrm{~Hz}$-Versuche vor. Die $1 \mathrm{~Hz}-$ Versuche wurden bei Erreichen des Durchläuferniveaus beendet. Die Ermüdungsbruchdehnungen streuen beträchtlich. Sie unter- und überschreiten die Bruchdehnungen bei monoton steigender Beanspruchung. Die mittlere Ermüdungsbruchdehnung der Serie I ist kleiner als die der Serie II. Die Dehnungsdifferenz beträgt 0,3 \%. Dies entspricht nicht dem erwarteten Einfluss aus der verminderten Kriechfähigkeit des Betons infolge der unterschiedlichen Betonalter. Bei dieser Beanspruchungsgruppe ist aber zu bedenken, dass die mittlere Bruchlastwechselzahl, und dementsprechend die mittlere Versuchsdauer der Serie II, um den Faktor zwei größer war als die der Serie I.

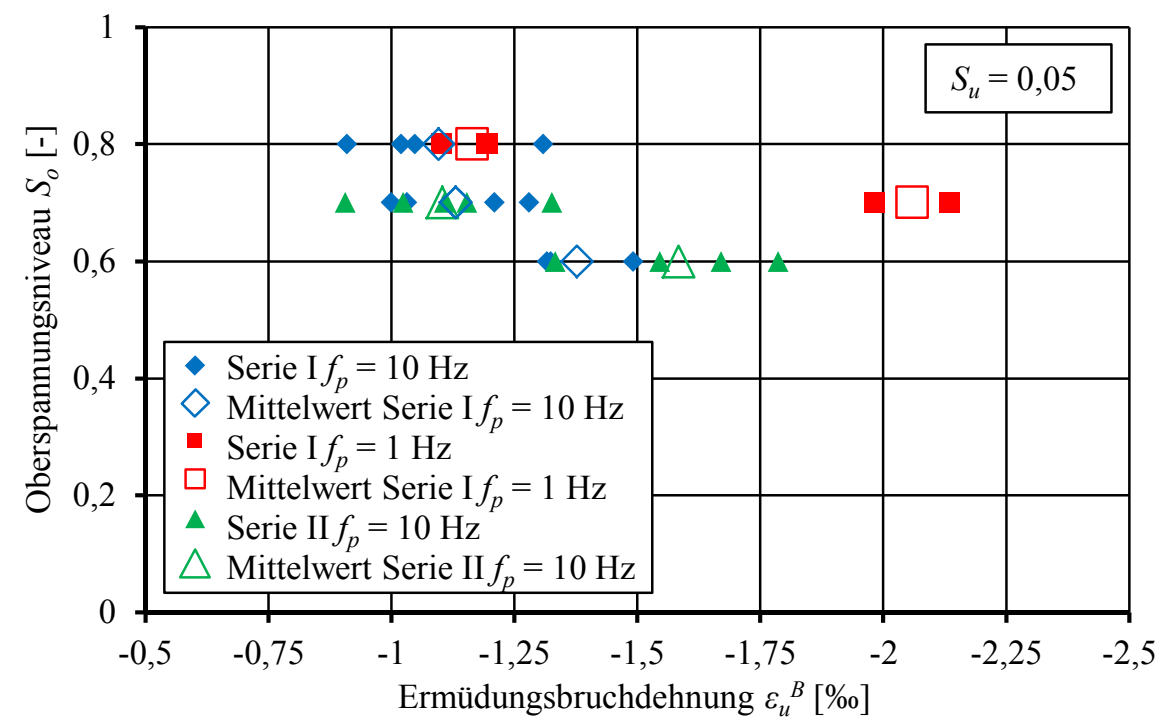

Abbildung 5.13: Ermüdungsbruchdehnungen auf dem Unterspannungsniveau in Abhängigkeit vom Oberspannungsniveau

Werden die Ermüdungsbruchdehnungen auf dem Unterspannungsniveau $\varepsilon_{u}{ }^{B}$ betrachtet, so ergibt sich auf den ersten Blick ein ,geordnetereres“ Bild gegenüber den Ermüdungsbruchdehnungen auf dem Oberspannungsniveau. Dabei ist die Streubreite der Dehnungswerte mit 1,23\%o (von $-0,91 \%$ bis $-2,14 \%$ ) unwesentlich kleiner als auf dem Oberspannungsniveau mit 1,34 \%o (von -2,97\%o bis -4,31\%). Die Dehnungsdifferenz zwischen den $1 \mathrm{~Hz}-$ Versuchen und den $10 \mathrm{~Hz}$-Versuchen auf dem Oberspannungsniveau von $S_{o}=0,70$ ist auch weiterhin präsent. Auf den einzelnen Beanspruchungsniveaus und den jeweiligen Belastungsfrequenzen streuen die Ermüdungsbruchdehnungen des Unterspannungsniveaus aber deutlich weniger als auf dem Oberspannungsniveau. Zudem liegen die gemittelten Ermüdungsbruchdehnungen des Unterspannungsniveaus zwischen den Versuchsserien I und II dichter zusammen als die gemittelten Ermüdungsbruchdehnungen des Oberspannungsniveaus. So liegt die mittlere Dehnungsdifferenz zwischen den Versuchsserien I und II für $S_{o}=0,60$ auf dem Unterspannungsniveau bei $0,2 \%$, während sie auf dem Oberspannungsniveau bei 0,3 \%o liegt. 
Für $S_{o}=0,70$ beträgt die Dehnungsdifferenz auf dem Unterspannungsniveau vernachlässigbare $0,03 \%$, während sie auf dem Oberspannungsniveau 0,14 \%o beträgt. Daraus ist zu schließen, dass die Streuungen auf dem Oberspannungsniveau zum Teil aus den Streuungen der Betonsteifigkeit bzw. aus dem elastischen Dehnungsanteil resultieren.

Die Ermüdungsbruchdehnungen auf dem Oberspannungsniveau und dem Unterspannungsniveau weisen gegenüber der logarithmierten Bruchlastwechselzahl keine signifikante Abhängigkeit auf, wie den Abbildungen A.2.38 und A.2.39 des Anhangs entnommen werden kann. Die Bestimmtheitsmaße der dargestellten Ausgleichsgeraden liegen für die Ermüdungsbruchdehnungen auf dem Oberspannungsniveau bei $R^{2}=0,01$ und für das Unterspannungsniveau bei $R^{2}=0,35$.

\subsubsection{Viskoses Verformungsverhalten}

Es wurden insgesamt 8 Kriechversuche auf Spannungsniveaus von $40 \%$ (2 Versuche), $50 \%$ (4 Versuche) und $60 \%$ (2 Versuche) der mittleren Zylinderdruckfestigkeit durchgeführt. Die Betonproben wurden in dem Kriechstand in der Regel 2 bis 3 Wochen belastet. Die Versuche erfolgten nacheinander, wodurch sich das Alter der Proben bei Belastungsbeginn stark unterschied. In der Abbildung 5.14 werden die Kriechkurven für das Beanspruchungsniveau von $60 \%$ der Zylinderdruckfestigkeit dargestellt. Die Verläufe für die Beanspruchungsniveaus von $40 \%$ und $50 \%$ der Zylinderdruckfestigkeit können dem Anhang A.3 entnommen werden. Da die Ermüdungsversuche mehrheitlich innerhalb von 36 Stunden bzw. 1,5 Tagen versagten, wurde diese Zeitspanne für die folgende Betrachtung der Kriechverformungen gewählt.

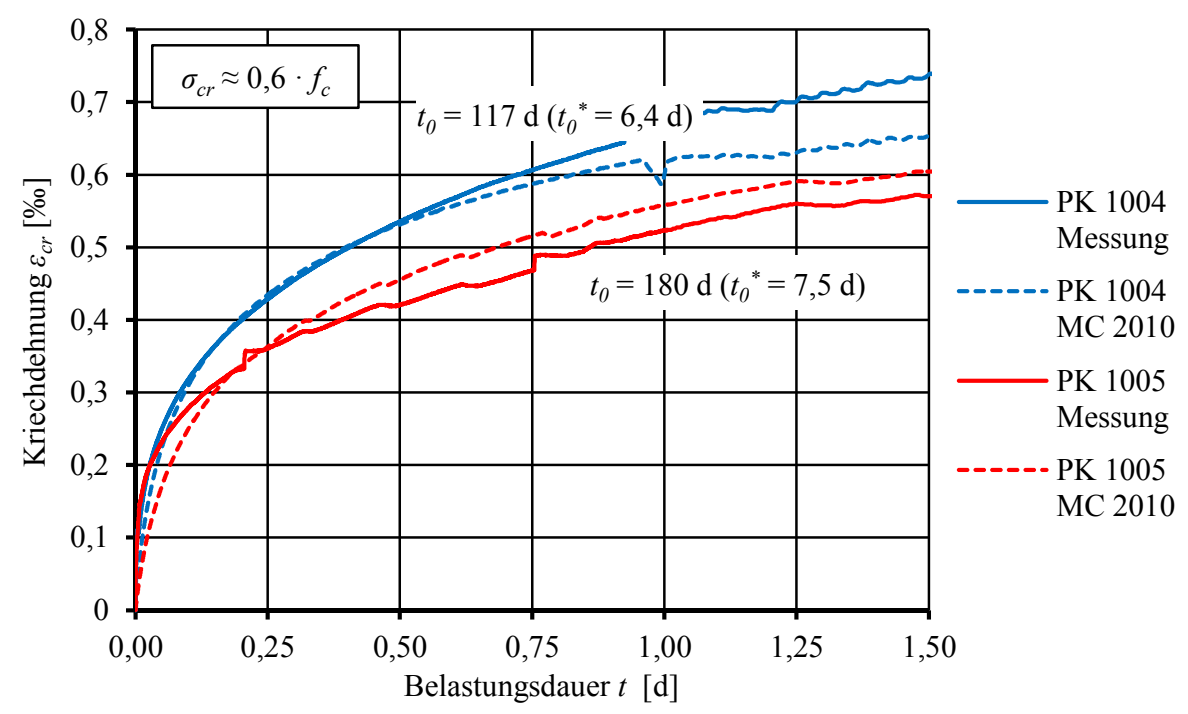

Abbildung 5.14: Kriechkurven für ein Beanspruchungsniveau von $\sigma_{c r}=0,6 \cdot f_{c}$

Die aufgezeichneten Verformungsverläufe der Kriechproben sind als durchgezogene Kurvenverläufe dargestellt. Die Schwankungen sind auf Temperatur- und Luftfeuchteschwankungen sowie auf gelegentliches Korrigieren des Belastungsdruckes zurückzuführen. Bei den dargestellten Verformungsverläufen handelt es sich ausschließlich um 
den Verformungsanteil nach Aufbringen der Dauerlast auf die Probekörper. Dieser nimmt mit zunehmendem Betonalter erwartungsgemäß ab. Des Weiteren werden die rechnerisch ermittelten Kriechkurven gestrichelt dargestellt. Diese wurden auf Grundlage des Formalapparats des Model Code 2010 ermittelt. Der Elastizitätsmodul wurde in den Berechnungen entsprechend eines C50/60 zu 38.629 N/mm² angenommen (Model Code 2010). Um eine gute Übereinstimmung zwischen dem realen Kriechverlauf und den rechnerischen Verformungen zu erzeugen, musste das Betonalter bei Belastungsbeginn für die Nachrechnung modifiziert werden. In diesem Zusammenhang werden die realen Betonalter $t_{0}$ und die rechnerisch angesetzten Betonalter $t_{0}{ }^{*}$ in Abbildung 5.15 grafisch gegenübergestellt. Daraus konnte ein linearer Zusammenhang zwischen realem und rechnerischem Betonalter abgeleitet werden, der sich zu

$$
t_{0}^{*}=0,022 \cdot t_{0}+3,823
$$

ergab. Das Bestimmtheitsmaß von $R^{2}=0,928$ deutet auf eine gute Näherung hin.

Der mathematische Zusammenhang ermöglicht für den untersuchten Beton die Kriechverformungen mit dem Formelapparat des Model Code 2010 für unterschiedliche Belastungszeitpunkte und Beanspruchungsniveaus $\mathrm{zu}$ berechnen. Im Vergleich zum realen Belastungsalter wird damit ein sehr junger Beton beschrieben. Die Datenauswertung zeigte, dass sich bei Betrachtung der Kriechverläufe über einen längeren Beanspruchungszeitraum (2 bis 3 Wochen) das rechnerische Betonalter dem realen Betonalter annäherte. Da aber insbesondere die viskosen Verformungen in den ersten Stunden der Belastungsphase beschrieben werden sollen, wurde trotz der großen Differenzen zwischen den beiden Zeitpunkten dieser mathematische Zusammenhang herangezogen.

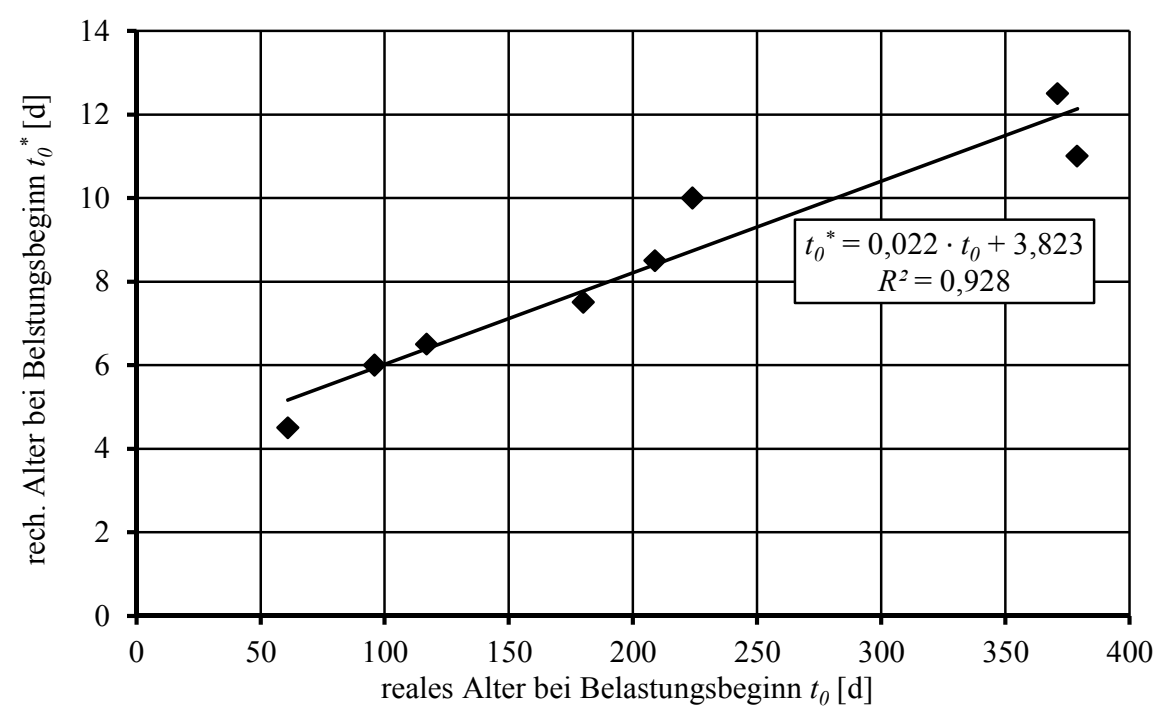

Abbildung 5.15: Verhältnis reales und rechnerisches Betonalter 


\section{Auswertung der Dehnungsanteile}

In diesem Kapitel werden die aufgezeichneten Dehnungsverläufe der ermüdungsbeanspruchten Betonprobekörper gemäß der additiven Modellvorstellung analysiert. Es erfolgt die Trennung hinsichtlich elastischer, thermischer, viskoser und schädigungsinduzierter Dehnungen. Die Bestimmung der einzelnen Dehnungsanteile erfolgt auf der Grundlage des in Kapitel 3 entwickelten additiven Dehnungsmodells und den in Kapitel 4 abgeleiteten kriechaffinen Beanspruchungsniveaus.

\subsection{Elastischer Dehnungsanteil}

Der elastische Dehnungsanteil entspricht der Dehnungsdifferenz zwischen dem Oberspannungsniveau und dem Entlastungsniveau. Das Dehnungsmaß zwischen Oberspannungsniveau und Unterspannungsniveau kann den Messwerten direkt entnommen werden, der Anteil zwischen Unterspannungsniveau und Entlastungsniveau ist rechnerisch zu bestimmen.

Ein Federelement repräsentiert den elastischen Dehnungsanteil in der rheologischen Modellvorstellung, wobei der Sekantenmodul des Entlastungsastes dessen Federsteifigkeit charakterisiert, vgl. Abbildung 3.4. Diese Betrachtungsweise vernachlässigt den gekrümmten Verlauf des Entlastungsastes. Die Berechnung der Dehnungsdifferenz zwischen Unterspannungsniveau und Entlastungsniveau mithilfe des Sekantenmoduls verursacht somit einen Fehler, der nachfolgend quantifiziert wird. In diesem Zusammenhang stellt die Abbildung 6.1 einzelne Lastzyklen des ermüdungsbeanspruchten Probekörpers PK 018 dar (durchgezogene Linien). Der Probekörper wurde bei einem Oberspannungsniveau von $S_{o}=0,70$, einem Unterspannungsniveau von $S_{u}=0,05$ und einer Belastungsfrequenz von $f_{p}=1 \mathrm{~Hz}$ belastet. Der Probekörper versagte nach 118.044 Lastwechseln (LW). Im Weiteren sind in der Grafik die Sekanten der Entlastungsäste dargestellt (gestrichelte Linien). Die Schnittpunkte dieser Geraden mit der Abszisse entsprechen den Dehnungswerten, die in dem vorgeschlagenen Dehnungsmodell auf dem Entlastungsniveau berücksichtigt werden.

Das reale Dehnungsverhalten des Betons zwischen Unterspannungsniveau und Entlastungsniveau wird auf der Grundlage quadratischer Ausgleichkurven prognostiziert (strich-punktierte Linien). Die Ausgleichskurven wurden per Best-Fit-Analyse an den Verlauf der Entlastungsäste zwischen Oberspannungsniveau und Unterspannungsniveau angepasst. Wird nun angenommen, dass diese Ausgleichskurven dem realen Dehnungsverhalten des Betons entsprechen, so resultiert der Dehnungswert auf dem Entlastungsniveau aus dem Schnittpunkt der Ausgleichskurve mit der Abszisse.

Auf dieser Grundlage kann der elastische Dehnungsanteil für beide Betrachtungsvarianten bestimmt werden. Der elastische Dehnungsanteil, ermittelt auf Basis des Sekantenmoduls, wird 
im Mittel um etwa $5 \%$ unterschätzt, im Vergleich zum elastischen Dehnungsanteil, ermittelt auf Basis der quadratischen Ausgleichskurve. Diese vereinfachte Abschätzung liefert bei dem gewählten Unterspannungsniveau von $S_{u}=0,05$ zufriedenstellende Ergebnisse. Es ist aber darauf hinzuweisen, dass der angenommene, parabelförmige Verformungsverlauf zwischen Entlastungsniveau und Unterspannungsniveau nur eine modellmäßige Näherung ist. Der tatsächliche Verlauf ist unbekannt. Der Fehler kann real sowohl größer als auch kleiner sein.

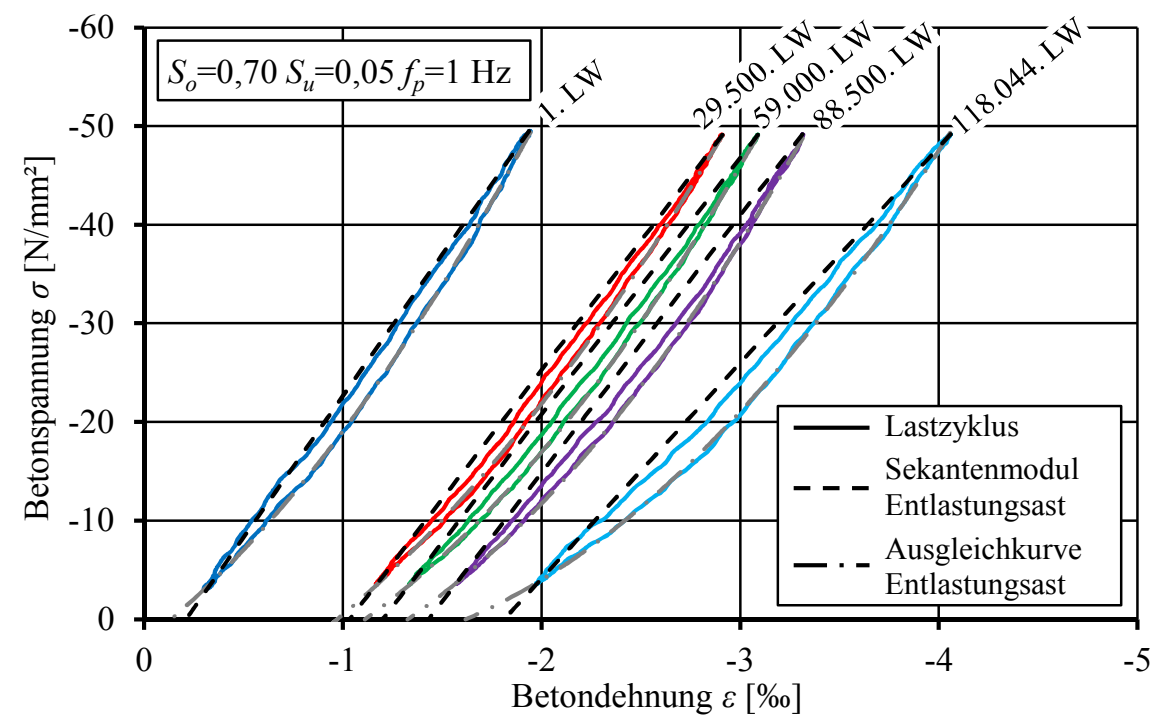

Abbildung 6.1: Ausgewählte Lastzyklen eines ermüdungsbeanspruchten Probekörpers

Die Abbildung 6.2 stellt die elastischen Dehnungen nach dem ersten Lastwechsel $\varepsilon_{e l}{ }^{0}$ und unmittelbar vor dem Versagen der Betonkörper $\varepsilon_{e l}^{B}$ dar. Die elastischen Dehnungswerte wurden mithilfe der Sekantenmodule aus Abschnitt A.2.3 berechnet und sind in Abschnitt A.2.4 ergänzend dokumentiert. Der elastische Dehnungsanteil nimmt mit steigendem Oberspannungsniveau zu. Zudem wachsen die elastischen Dehnungen zwischen Versuchsbeginn und Versuchsende entsprechend der abnehmenden Sekantensteifigkeit an. Die Dehnungszunahme ist bei den niedrigeren Oberspannungsniveaus größer als bei den höheren Oberspannungsniveaus. Dies ist auf die ausgeprägtere Steifigkeitsdegradation bei den niedrigeren Oberspannungsniveaus zurückzuführen. Hierdurch nimmt die Spannweite der elastischen Dehnungen zu Versuchsende ab. Während sich die elastischen Dehnungen bei Versuchsbeginn auf einen Bereich von -1,52 \%o bis -2,61 \%o erstrecken, nimmt diese Spannweite zu Versuchsende auf einen Bereich von -2,22 \%o bis $-3,21 \%$ ab. Die Spanne der elastischen Dehnungen sinkt somit von 1,09 \%o auf 1,01 \%o.

Der elastische Dehnungsanteil dominiert die gemessenen Ermüdungsbruchdehnungen der Betonprobekörper. Unmittelbar vor dem Versagen der Probekörper liegt dieser bei den Ermüdungsversuchen mit den geringeren Versuchsdauern ( $<2$ Stunden) bei etwa $80 \%$ der gemessenen Ermüdungsbruchdehnungen und nimmt in Abhängigkeit von der Versuchsdauer auf bis zu $60 \% \mathrm{ab}$, vgl. Abbildung 6.3. Damit treten viskose und schädigungsinduzierte Dehnungen auf, deren Anteile in Summe bei bis zu 40 \% der gemessenen Ermüdungsbruchdehnungen liegen. Es ist $\mathrm{zu}$ beachten, dass die auftretenden Temperaturdehnungen die messbaren 
Ermüdungsbruchdehnungen reduzieren, wodurch sich die prozentuale Verteilung zugunsten des elastischen Dehnungsanteils verschiebt.

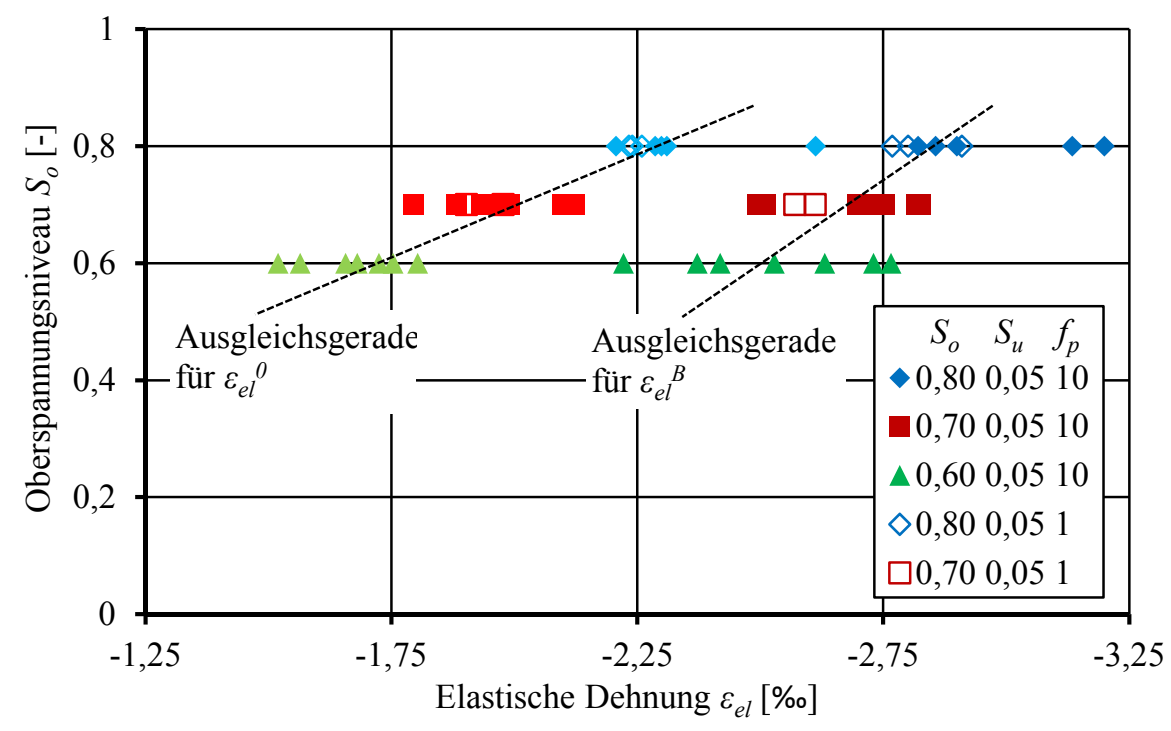

Abbildung 6.2: Elastische Dehnungsanteile zu Versuchsbeginn und Versuchsende

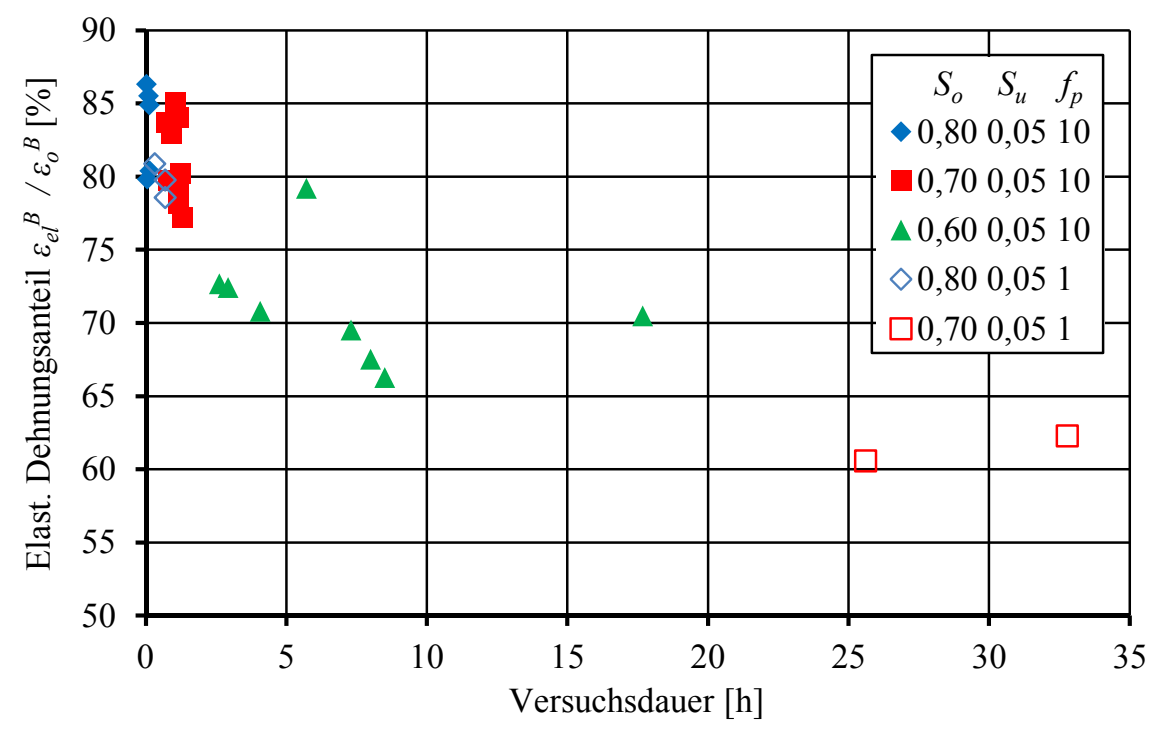

Abbildung 6.3: Prozentualer Anteil der elastischen Dehnungen an den gemessenen Ermüdungsbruchdehnungen

\subsection{Thermischer Dehnungsanteil}

Während der Versuchsdurchführung erwärmen sich die Probekörper in Abhängigkeit von der Spannungsamplitude und der Belastungsfrequenz. Der Temperaturanstieg wurde an drei Punkten auf der Oberfläche der Probekörper erfasst, vgl. Abschnitt 5.2.3. Um die auftretenden Temperaturdehnungen abzuschätzen, ist aus den punktuellen Temperaturmessungen eine mittlere, dehnungswirksame Probekörpertemperatur abzuleiten. In (Elsmeier, 2015) wurde die Probentemperatur im Kern und an der Oberfläche einer ermüdungsbeanspruchten Betonprobe erfasst. Das Verhältnis aus Kerntemperaturänderung zu Oberflächentemperaturänderung auf 
halber Probenhöhe ergab sich zu $\Delta T_{\text {Kern }} / \Delta T_{\text {außen }}=1,3$. Im Weiteren wurden Wärmebilder einer ermüdungsbeanspruchten Betonprobe aufgenommen. Im mittleren Drittel der Probenhöhe stellte sich eine relativ gleichmäßige Oberflächentemperatur ein, vgl. Abbildung 2.26. Zur Berechnung der mittleren, dehnungswirksamen Probentemperatur wird die gemessene Oberflächentemperatur auf halber Probenhöhe über das mittlere Drittel der Probenhöhe als konstant angenommen. Das Verhältnis aus Kerntemperatur zu Oberflächentemperatur wird in diesem Bereich zu 1,3 angenommen. Im oberen und unteren Drittel der Probenhöhe wird eine linear abnehmende Oberflächentemperatur bis auf die gemessenen Temperaturen im oberen und unteren Probenbereich angenommen. An den Stirnflächen der Proben wird der Verhältniswert aus Kerntemperatur und Oberflächentemperatur durch die Druckplatten der Prüfmaschine homogenisiert, sodass er zu 1,0 angenommen wird. Das berücksichtigte Temperaturprofil wird in Abbildung 6.4 dargestellt.

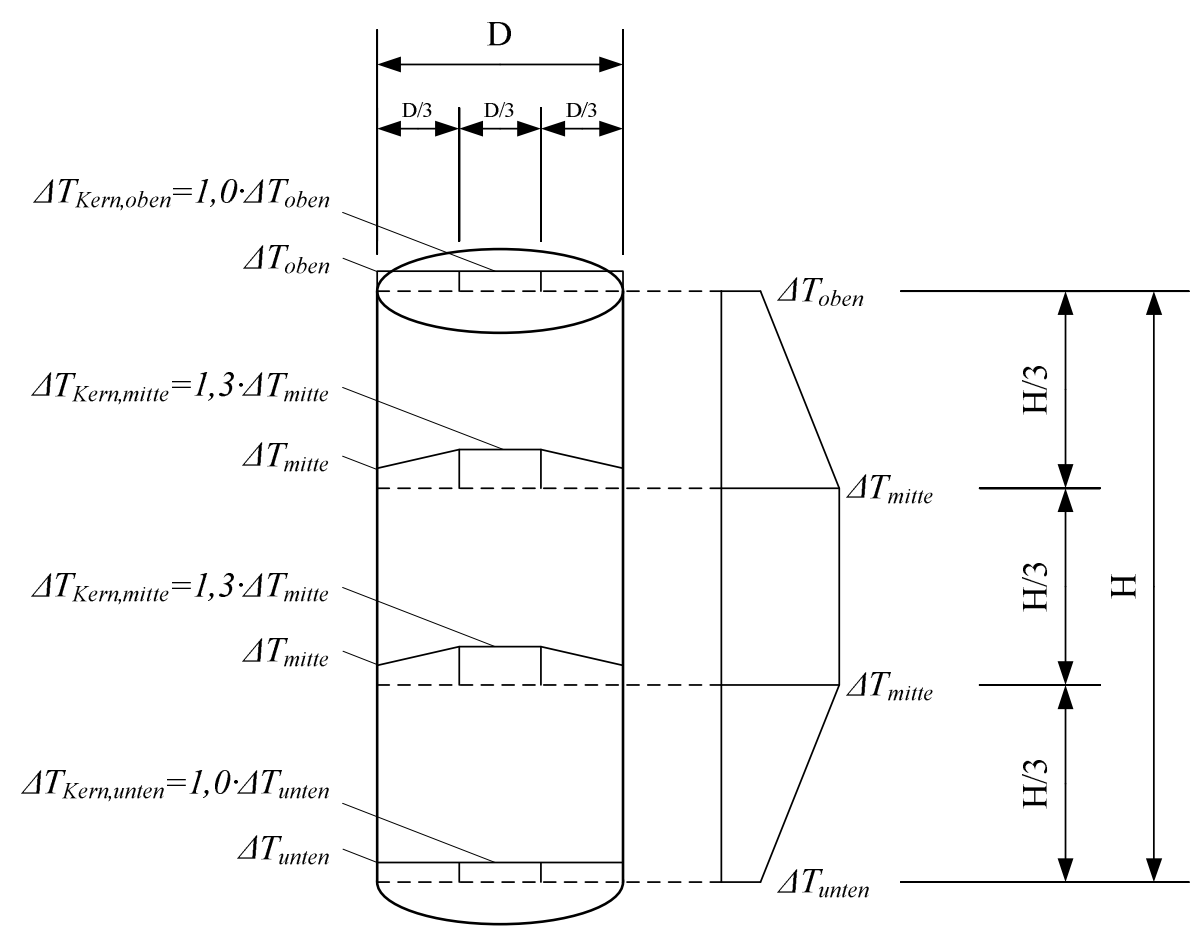

Abbildung 6.4: Angenommenes Temperaturprofil zur Berechnung der mittleren Probekörpertemperatur

Auf Grundlage des angenommen Temperaturprofils ergibt sich die mittlere Temperaturänderung zwischen Versuchsbeginn und Versuchsende anhand der gemessenen Temperaturänderungen in der Probenmitte und im oberen und unteren Randbereich zu:

$$
\Delta T_{\text {dehn }}=0,183 \cdot \Delta T_{\text {oben }}+0,767 \cdot \Delta T_{\text {mitte }}+0,183 \cdot \Delta T_{\text {unten }}
$$

Damit errechnet sich die mittlere Temperaturdehnung der Probekörper zu:

$$
\varepsilon_{t}=\alpha_{T} \cdot \Delta T_{d e h n}
$$

Der Anhang A.2.2 listet die mittleren, dehnungswirksamen Temperaturänderungen und die resultierenden Temperaturdehnungen bei einem Wärmeausdehnungskoeffizient von $\alpha_{T}=1 \cdot 10^{-5}$ 
tabellarisch auf. Darüber hinaus werden die Temperaturdehnungen in Abbildung 6.5 grafisch dargestellt.

Die $1 \mathrm{~Hz}$-Versuche erwärmen sich nur geringfügig, die auftretenden Temperaturdehnungen liegen in der Größenordnung von bis zu 0,1\%o. Dies trifft ebenfalls für die $10 \mathrm{~Hz}-$ Versuche bei dem Oberspannungsniveau von $S_{o}=0,80$ zu. Bei den $10 \mathrm{~Hz}$-Versuchen mit den niedrigeren Oberspannungsniveaus $\left(S_{o}=0,7\right.$ und 0,6$)$ führt die größere Versuchsdauer $\mathrm{zu}$ höheren Probekörpererwärmungen und damit zu größeren Temperaturdehnungen. Wie in Abbildung 6.6 dargestellt liegt der betragsmäßige Anteil der Temperaturdehnungen an den gemessenen Ermüdungsbruchdehnungen auf dem Oberspannungsniveau bei bis zu $12 \%$. Die auftretenden Temperaturdehnungen besitzen somit einen nicht $\mathrm{zu}$ vernachlässigen Anteil an den Gesamtverformungen.

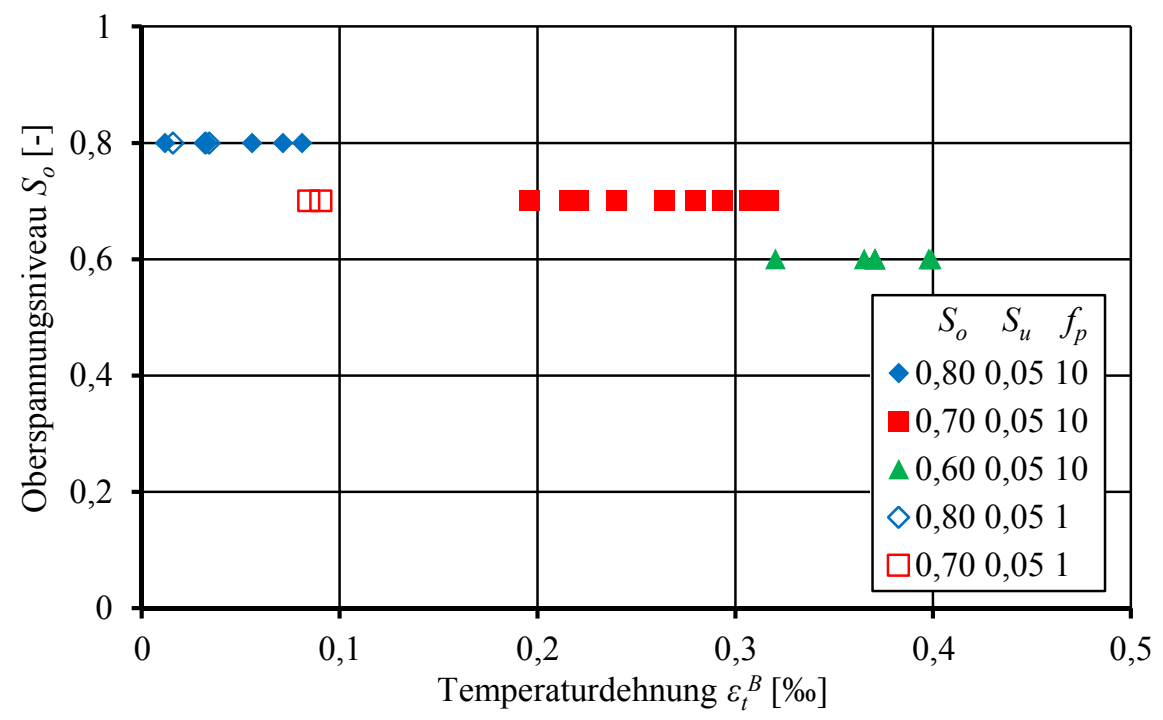

Abbildung 6.5: Temperaturdehnungen der Ermüdungsproben bei Versuchsende

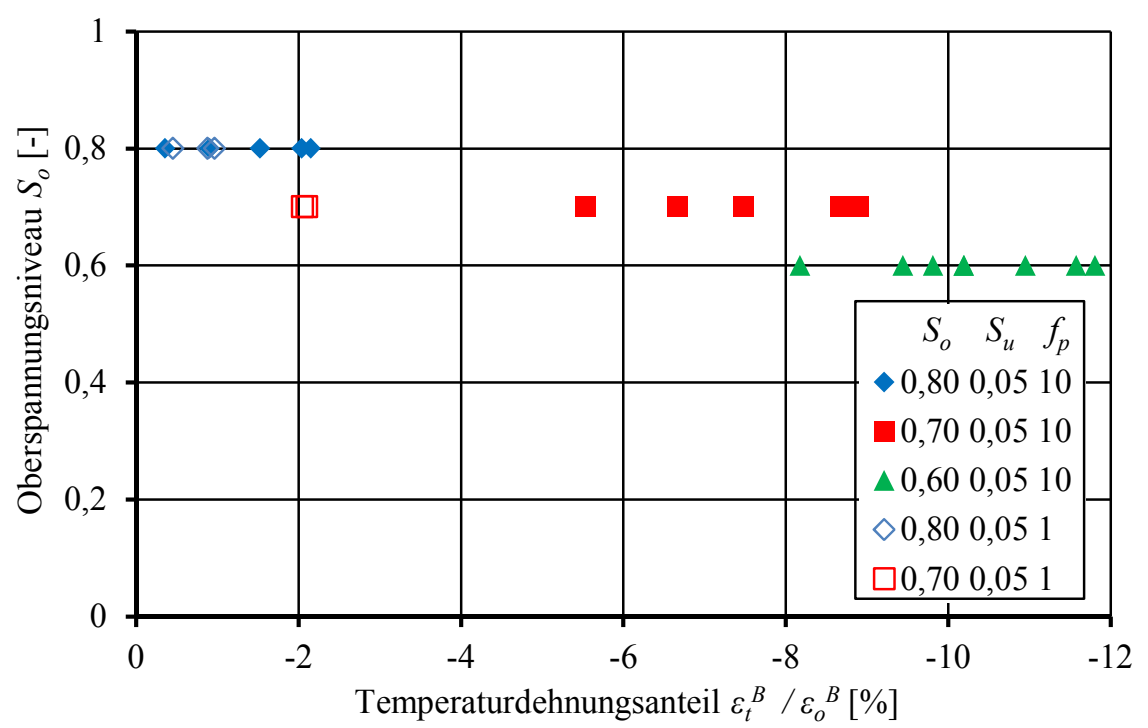

Abbildung 6.6: Prozentualer Anteil der Temperaturdehnungen an den gemessenen Ermüdungsbruchdehnungen 
Werden die gemessenen Ermüdungsbruchdehnungen aus Abbildung 5.12 um die berechneten Temperaturdehnungen korrigiert, ergibt sich die in Abbildung 6.7 dargestellte Dehnungsverteilung. Bei den Temperaturdehnungen handelt es sich um eine Ausdehnung der Probekörper, die die gemessenen Betonstauchungen im Ermüdungsversuch reduzieren. Die Betondehnungen sind in Abbildung 6.7 somit gegenüber Abbildung 5.12 vergrößert. In der Grafik ist wie zuvor der Bereich der Bruchdehnungen infolge monoton steigender Beanspruchung grau hinterlegt. Auffällig ist, dass nur noch ein Probekörper diesen Bereich unterschreitet. Alle anderen Probekörper liegen in diesem oder übertreffen diesen Bereich. Zudem hat die Streubreite der Dehnungswerte abgenommen. Die minimale gemessene Ermüdungsbruchdehnung auf dem Oberspannungsniveau lag bei $-2,97 \%$ und die Maximale bei $-4,31 \%$. Daraus ergab sich eine Differenz von 1,34 \%. Durch die Korrektur der Dehnungswerte um die Temperaturdehnungen resultieren eine minimale Dehnung von $-3,23 \%$ und eine maximale Dehnung von $-4,40 \%$. Die Differenz beträgt 1,16 \%o. Die Spanne der Ermüdungsbruchdehnungen nimmt um rd. $13 \%$ ab.

Die Dehnungsdifferenz von 0,88 \% zwischen den $10 \mathrm{~Hz}$ - und den $1 \mathrm{~Hz}-$ Versuchen auf dem Oberspannungsniveau von $S_{o}=0,70$ nimmt durch die Temperaturkompensation ab. Die $10 \mathrm{~Hz}-$ Versuche erwärmen sich stärker und dehnen sich dementsprechend mehr aus als die $1 \mathrm{~Hz}-$ Versuche. Die mittlere Dehnungsdifferenz der Versuchsreihen reduziert sich von $0,88 \%$ auf $0,72 \%$. Das entspricht einer Änderung von rd. $20 \%$. Auf dem Oberspannungsniveau von $S_{o}=0,80$ änderte sich die Dehnungsdifferenz kaum. Die $10 \mathrm{~Hz}$ - und die $1 \mathrm{~Hz}-$ Versuche erwärmen sich etwa gleich stark. Gleiches gilt für die Ermüdungsversuche auf dem Oberspannungsniveau von $S_{o}=0,60$ der Versuchsserien I und II bei einer Belastungsfrequenz von $10 \mathrm{~Hz}$.

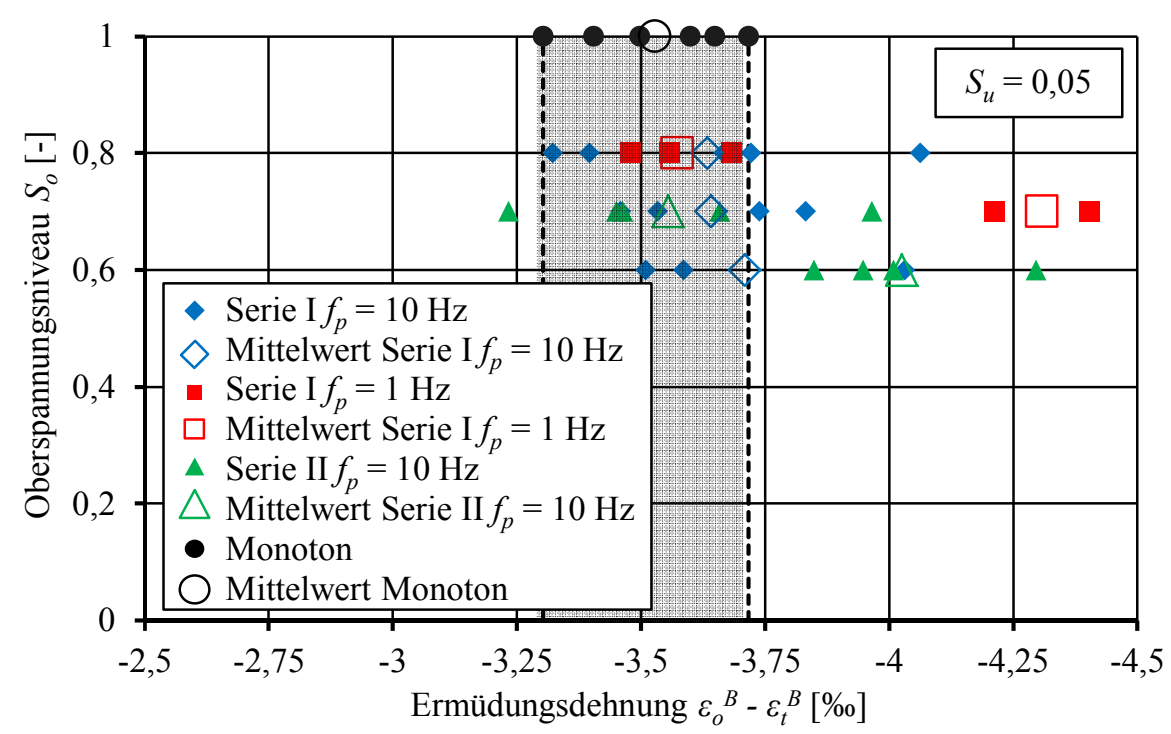

Abbildung 6.7: Ermüdungsbruchdehnungen korrigiert um die Temperaturdehnungen

Die Mittelwerte der Ermüdungsbruchdehnungen aller Versuchsreihen liegen nach der Temperaturkompensation über der mittleren Bruchdehnung der monoton beanspruchten Druckversuche. Die gemittelte, temperaturkompensierte Ermüdungsbruchdehnung aller Versuchskörper beträgt nun $-3,73 \%$ und ist damit $6 \%$ größer als die Bruchdehnung der Druckversuche. Zuvor waren die Werte nahezu gleich groß. 


\subsection{Viskoser Dehnungsanteil}

Das kriechaffine Spannungsniveau $S_{c r}$ der ermüdungsbeanspruchten Betonproben resultiert aus dem Ober- und Unterspannungsniveau der sinusförmigen Beanspruchung. Von der Belastungsfrequenz wird das Ergebnis entsprechend Abschnitt 4.6 nicht beeinflusst. Auf Grundlage von Abbildung 4.16 ergeben sich die kriechaffinen Beanspruchungsniveaus der untersuchten Beanspruchungsverläufe zu den in Tabelle 6.1 angegebenen Werten. Mithilfe der kriechaffinen Beanspruchungsniveaus, dem Formalismus des Model Code 2010 und dem modifizierten Betonalter entsprechend Gleichung 5.3 kann die viskose Verformungsentwicklung der Betonproben im Ermüdungsversuch abgeschätzt werden. Die Abbildung 6.8 und die Abbildung 6.9 stellen die zugehörigen Kriechkurven für das mittlere modifizierte Betonalter der Versuchsserie I mit $t_{0}{ }^{*}=5,2$ Tagen und der Versuchsserie II mit $t_{0}{ }^{*}=6,9$ Tagen dar (durchgezogene Linien). Das angesetzte Betonalter beeinflusst die Kriechfähigkeit des Betons wesentlich. Die Kriechverformungen der Serie II sind im Schnitt um rd. $20 \%$ kleiner als die der Serie I.

Tabelle 6.1: Kriechaffine Spannungsniveaus der Ermüdungsversuche

\begin{tabular}{|c|c|c|}
\hline $\boldsymbol{S}_{\boldsymbol{o}}=\boldsymbol{\sigma}_{\boldsymbol{o}} / \boldsymbol{f}_{\boldsymbol{c}}$ & $\boldsymbol{S}_{\boldsymbol{u}}=\boldsymbol{\sigma}_{\boldsymbol{u}} / \boldsymbol{f}_{\boldsymbol{c}}$ & $\boldsymbol{S}_{\boldsymbol{c}}=\boldsymbol{\sigma}_{\boldsymbol{c}} / \boldsymbol{f}_{\boldsymbol{c}}$ \\
\hline 0,80 & 0,05 & 0,513 \\
\hline 0,70 & 0,05 & 0,447 \\
\hline 0,60 & 0,05 & 0,376 \\
\hline
\end{tabular}

Die viskosen Verformungen der Betonproben im Ermüdungsversuch wurden in Abhängigkeit vom Betonalter bei Versuchsbeginn und von ihrer Versuchslaufzeit berechnet. Die Versuchslaufzeit ergab sich aus dem Quotienten von Lastwechselzahl und Belastungsfrequenz. Die berechneten Verformungen sind in den nachfolgenden Abbildungen als Punkte visualisiert. Da das jeweilige Alter der Betonproben vom mittleren Betonalter der Versuchsserien abweicht, liegen die berechneten Verformungen der einzelnen Probekörper geringfügig oberhalb (Probekörper, die zu Beginn der Versuchsserien getestet wurden) oder unterhalb (Probekörper, die zum Ende der Versuchsserien getestet wurden) der dargestellten Kriechkurven.

Die viskosen Verformungen der Probekörper mit den längsten Versuchslaufzeiten liegen in der Größenordnung von $0,4 \%$ bis $0,5 \%$. Der Anteil der viskosen Verformungen an den gemessenen Ermüdungsbruchdehnungen auf dem Oberspannungsniveau liegt bei bis zu $11 \%$ und stellt damit eine nicht $\mathrm{zu}$ vernachlässigende Einflussgröße auf das Dehnungsverhalten des Betons im Ermüdungsversuch dar, vgl. Abbildung 6.10.

Bemerkenswert ist der Einfluss der Belastungsfrequenz, die bei den Versuchen der Serie I auf dem Spannungsniveau von $S_{o}=0,70$ und $S_{u}=0,05$ bzw. dem zugehörigen Kriechniveau von $S_{c r}=0,447$ sichtbar wird, vgl. Abbildung 6.8. Die Versuchslaufzeit der $10 \mathrm{~Hz}$-Versuche lag in der Größenordnung von 1 Stunde, während die $1 \mathrm{~Hz}-$ Versuche ca. 30 Stunden dauerten. Die Dehnungsdifferenz zwischen diesen Versuchsreihen liegt bei 0,25\%. Damit resultieren aus den variierenden Versuchslaufzeiten unterschiedlich große viskose Verformungen während der Ermüdungsversuche. Die in Abschnitt 5.2.5 beschriebene Dehnungsdifferenz zwischen den 
Ermüdungsbruchdehnungen auf dem Oberspannungsniveau von $0,88 \%$ zwischen den genannten Versuchsreihen besteht somit zu einem wesentlichen Anteil $(\approx 30 \%)$ aus der viskosen Verformungskomponente. Nach Korrektur der Dehnungswerte um die thermischen und viskosen Dehnungen nimmt die Differenz auf 0,4 \%o ab.

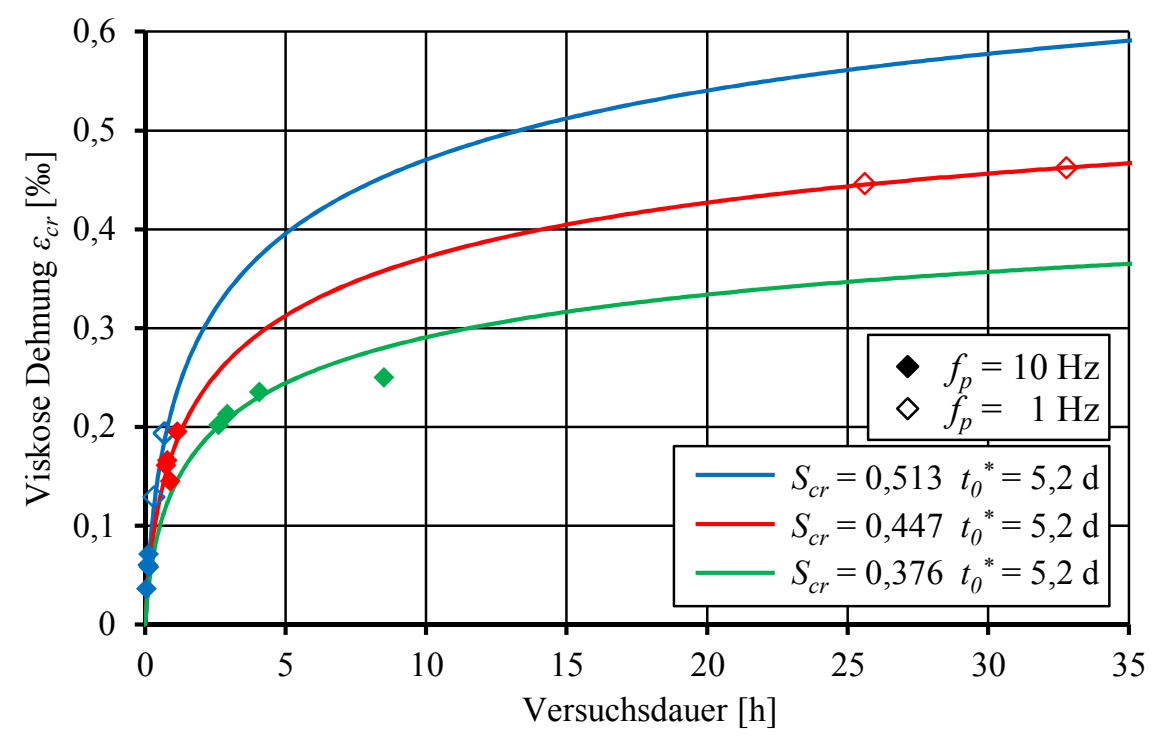

Abbildung 6.8: Viskose Dehnungen der Versuchsserie I

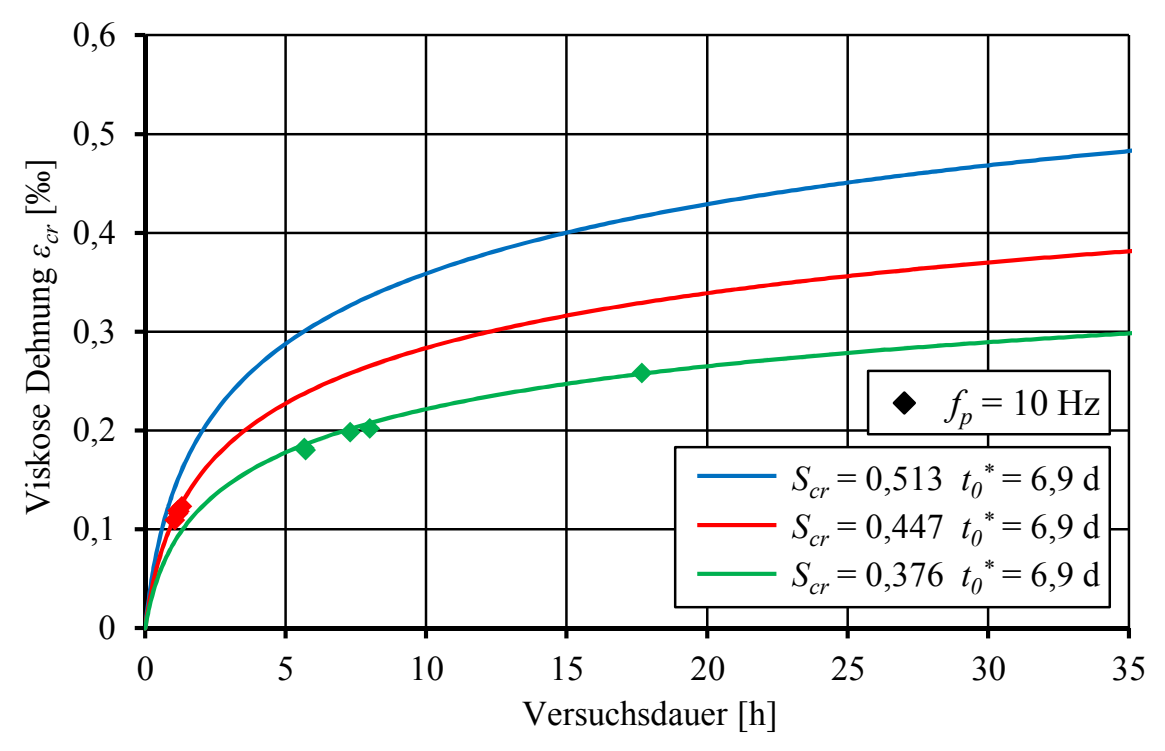

Abbildung 6.9: Viskose Dehnungen der Versuchsserie II

Werden die Versuche auf dem Spannungsniveau von $S_{o}=0,60$ und $S_{u}=0,05 \mathrm{bzw}$. dem zugehörigen Kriechniveau von $S_{c r}=0,376$ der Versuchsserien I und II gegenübergestellt, so ist die mittlere Versuchsdauer der Serie II doppelt so groß wie die der Serie I. Durch das höhere Betonalter der Serie II liegen die viskosen Verformungen jedoch etwa in der gleichen Größenordnung wie die der Serie I.

Die Abbildung 6.11 stellt die korrigierten Ermüdungsbruchdehnungen der Ermüdungsversuche dar. Die gemessenen Ermüdungsbruchdehnungen auf dem Oberspannungsniveau wurden um die 
Temperaturdehnungen und die viskosen Dehnungen korrigiert. Vergleicht man die Darstellung mit den gemessenen Ermüdungsbruchdehnungen aus Abbildung 5.12, ist die Spanne zwischen dem größten und dem kleinsten Dehnungswert sichtbar geschrumpft. Die gemessenen Dehnungswerte erstreckten sich auf einen Bereich von $-2,97 \%$ bis $-4,31 \%$. Nach der Korrektur der Dehnungswerte erstrecken sie sich auf einen Bereich von $-3,11 \%$ bis $-4,04 \%$. Die Streubreite der Dehnungen hat somit um fast $30 \%$ von 1,34\% auf 0,93\%o abgenommen. Insgesamt liegen rd. $60 \%$ der korrigierten Ermüdungsbruchdehnungen in dem Bereich der Bruchdehnungen infolge monoton steigender Beanspruchung. Die übrigen Ermüdungsbruchdehnungen liegen unterhalb oder auch oberhalb dieses Bereichs. Die mittlere, korrigierte Ermüdungsbruchdehnung aller Ermüdungsversuchskörper liegt bei $-3,56 \%$ und ist damit unwesentlich größer als die mittlere Bruchdehnung der Druckversuche mit -3,53\%o.

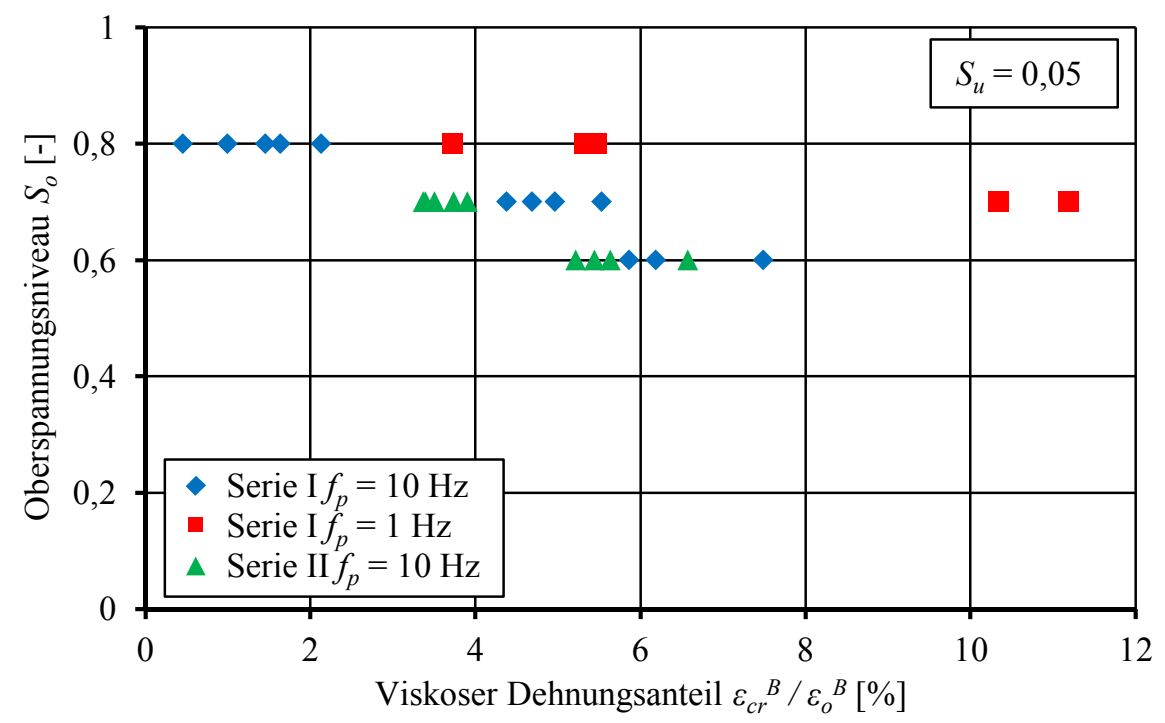

Abbildung 6.10: Prozentualer Anteil der viskosen Dehnungen an den gemessenen Ermüdungsbruchdehnungen

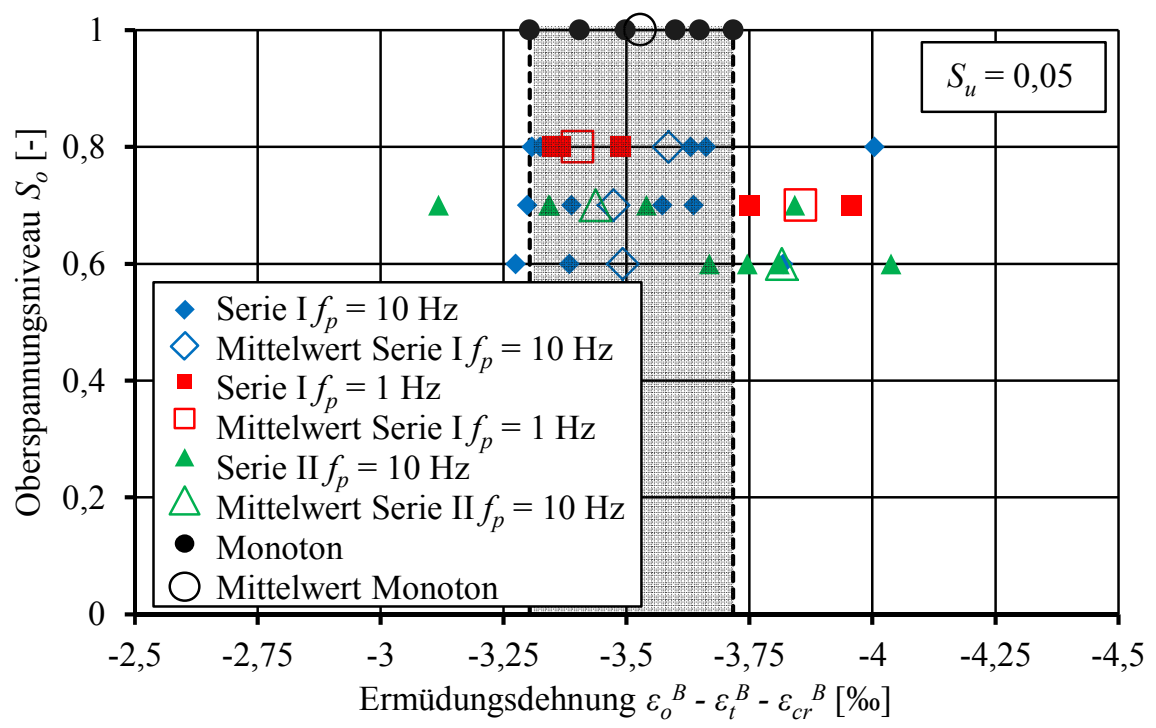

Abbildung 6.11: Ermüdungsbruchdehnungen nach der Korrektur um die thermischen und viskosen Dehnungen 


\subsection{Schädigungsinduzierter Dehnungsanteil}

Der schädigungsinduzierte Dehnungsanteil errechnet sich aus den Ermüdungsbruchdehnungen auf dem Entlastungsniveau $\varepsilon_{0 K N}$ abzüglich der im Ermüdungsversuch auftretenden Temperaturdehnungen $\varepsilon_{t}$ und viskosen Dehnungen $\varepsilon_{c r}$. Die berechneten schädigungsinduzierten Dehnungen der Versuchskörper sind in Abbildung 6.12 in Abhängigkeit vom Oberspannungsniveau dargestellt. Die Dehnungswerte sind scheinbar vom Oberspannungsniveau und der Belastungsfrequenz abhängig und nehmen mit abnehmenden Oberspannungsniveaus zu.

Werden zunächst ausschließlich die $10 \mathrm{~Hz}$-Versuche analysiert, ergeben sich bei den Versuchen der Serie II auf den Oberspannungsniveaus von $S_{o}=0,70$ und 0,60 im Mittel größere schädigungsinduzierte Dehnungen als bei den Versuchen der Serie I. Werden vor diesem Gesichtspunkt die Bruchlastwechselzahlen dieser Versuche betrachtet, weisen die Versuche der Serie II ebenfalls größere Werte auf als die Versuche der Serie I. In Kapitel 3 wurde die These geäußert, dass sich die schädigungsinduzierten Dehnungen vor allem in Abhängigkeit von der Bruchlastwechselzahl entwickeln. Diese These bestätigt sich nun. Die Versuche mit höheren Bruchlastwechselzahlen führen zu größeren schädigungsinduzierten Dehnungen als Versuche mit geringeren Bruchlastwechselzahlen. Die Ursache wird in der zunehmenden Zermürbung und Materialzerrüttung des Betons in Abhängigkeit von den aufgebrachten Lastwechseln gesehen. Dies gilt ebenso für die $1 \mathrm{~Hz}$ - und $10 \mathrm{~Hz}$-Versuche im Vergleich. Auf dem höchsten Beanspruchungsniveau von $S_{o}=0,80$ unterschreiten die gemittelten schädigungsinduzierten Dehnungen der $1 \mathrm{~Hz}-$ Versuche die der $10 \mathrm{~Hz}-$ Versuche. Dieser Trend ist bei den erreichten Bruchlastzahlen wiederzufinden. Bei dem Beanspruchungsniveau von $S_{o}=0,70$ sind bei den $1 \mathrm{~Hz}-$ Versuchen sowohl die mittlere schädigungsinduzierte Dehnung als auch die mittlere Bruchlastwechselzahl größer als bei den $10 \mathrm{~Hz}-$ Versuchen.

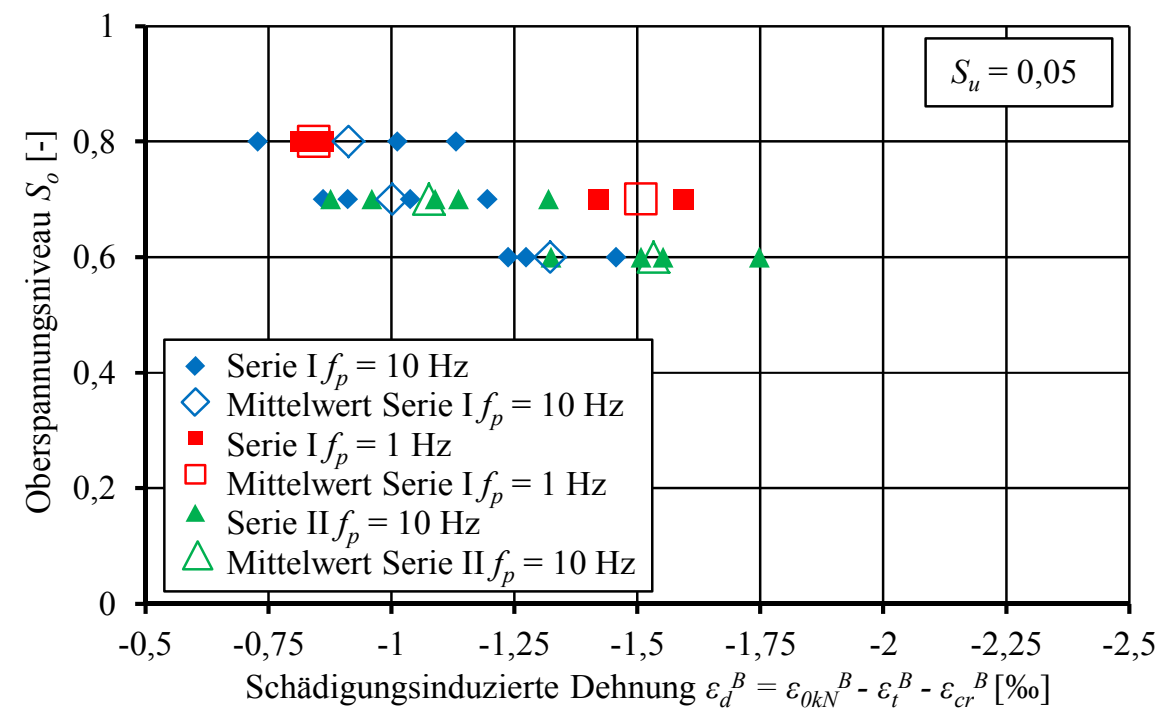

Abbildung 6.12: Schädigungsinduzierter Dehnungsanteil in Abhängigkeit vom Oberspannungsniveau

Dieser These folgend werden in Abbildung 6.13 die schädigungsinduzierten Dehnungen in Abhängigkeit von der logarithmierten Bruchlastwechselzahl dargestellt. Es wird ein linearer 
Zusammenhang sichtbar. Das Bestimmtheitsmaß der Ausgleichsgerade beträgt $R^{2}=0,68$. Das Ergebnis spiegelt damit den in Abschnitt 5.2.4 festgestellten linearen Zusammenhang zwischen Sekantensteifigkeit und logarithmierter Bruchlastwechselzahl wider. Die makroskopische Steifigkeitsdegradation kann als Indiz für die Gefügezerstörung im Betonkörper aufgefasst werden. Eine ausgeprägtere Gefügezerstörung resultiert dementsprechend auch in größeren schädigungsinduzierten Dehnungen wie bereits in Abschnitt 5.2.4 vermutet.

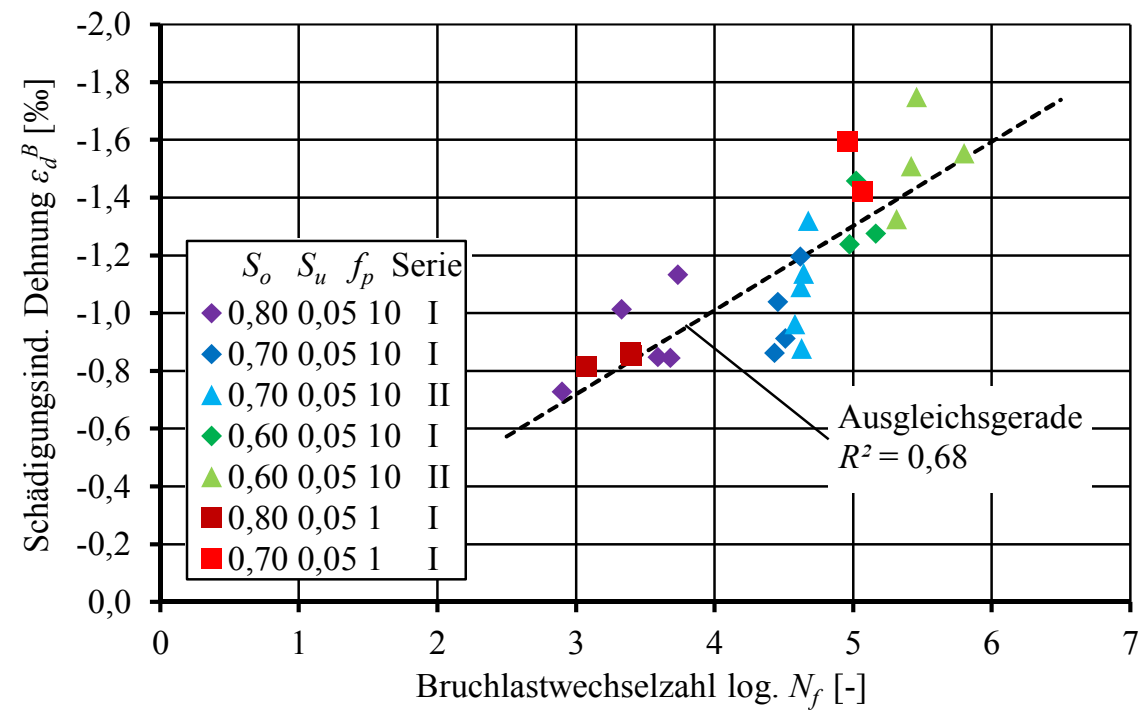

Abbildung 6.13: Schädigungsinduzierte Dehnungen in Abhängigkeit der logarithmierten Bruchlastwechselzahl

Die verbleibenden Sekantensteifigkeiten, unmittelbar vor dem Bruch der Betonproben, und die schädigungsinduzierten Dehnungen weisen folglich eine lineare Beziehung auf, vgl. Abbildung 6.14. Der lineare Zusammenhang ist mäßig ausgeprägt, da sich hier die Modellungenauigkeiten bei der Bestimmung des schädigungsinduzierten Dehnungsanteils und der Sekantensteifigkeit überlagern. Der lineare Zusammenhang bestätigt aber die These, dass der Sekantenmodul ein Indiz für die Gefügeschädigung darstellt und die Gefügeschädigung sich im schädigungsinduzierten Dehnungsanteil niederschlägt. Zudem entspricht dies der Annahme, dass sich die Anteile in Abhängigkeit von der Lastwechselzahl entwickeln. Damit wird das additive Dehnungsmodell bestätigt. Dieser Zusammenhang wäre ohne die Korrektur der im Ermüdungsversuch gemessenen Dehnungen um die auftretenden thermischen und viskosen Dehnungen nicht erfassbar gewesen. Somit bestätigen sich auch die getroffenen Modellannahmen zur Temperaturdehnung und zum kriechaffinen Beanspruchungsniveau bzw. zum viskosen Dehnungsanteil.

Die Wöhlerlinien von Beton werden gewöhnlich in Abhängigkeit der logarithmierten Bruchlastwechselzahl dargestellt. In dem untersuchten Lastwechselbereich stellen sich diese als lineare Beziehung dar, vgl. Abbildung 2.16. Durch die Korrektur der gemessenen Ermüdungsbruchdehnungen zeigt sich, dass der schädigungsinduzierte Dehnungsanteil des Betons dieses Materialverhalten widerspiegelt. 


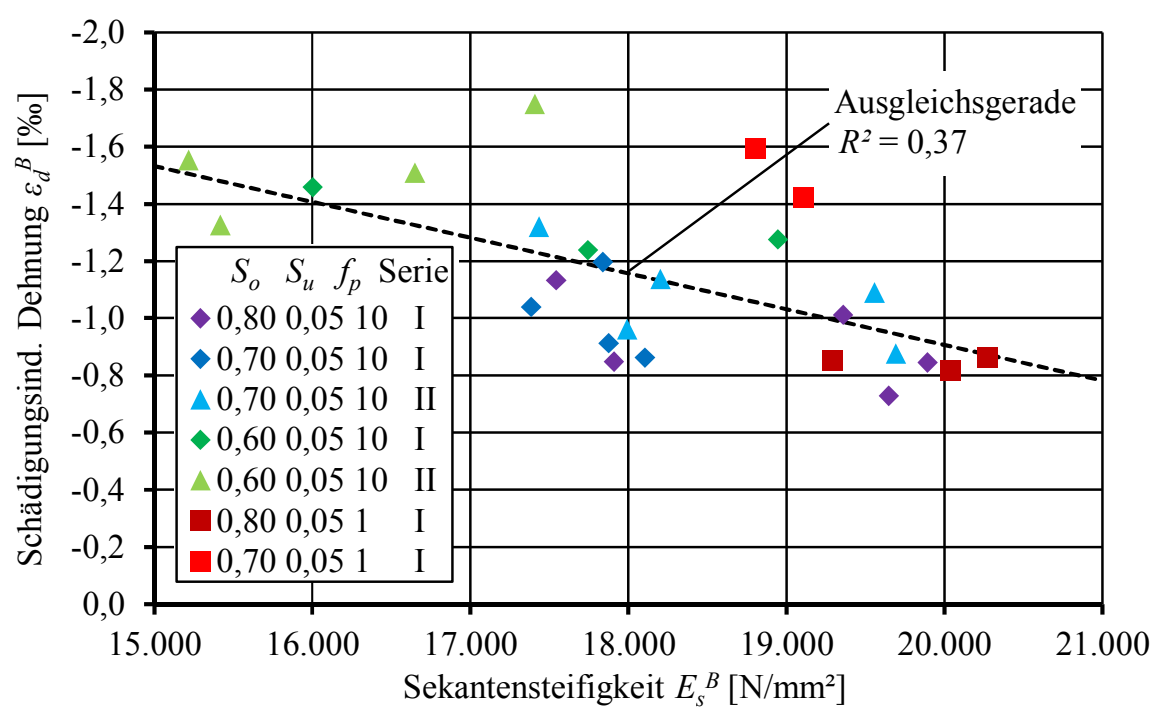

Abbildung 6.14: Schädigungsinduzierte Dehnungen in Abhängigkeit der Sekantensteifigkeit

\subsection{Dehnungsentwicklung}

Bislang wurden die Dehnungen unmittelbar vor dem Versagen der Probekörper betrachtet. Dabei entwickeln sich die elastischen, thermischen, viskosen und schädigungsinduzierten Dehnungen in Abhängigkeit von der Zeit und der Lastwechselzahl unterschiedlich voneinander. Vor diesem Hintergrund werden die Dehnungsanteile bei einem Oberspannungsniveau von $S_{o}=0,70$, einem Unterspannungsniveau von $S_{u}=0,05$ und einer Belastungsfrequenz von $f_{p}=1 \mathrm{~Hz}$ und $10 \mathrm{~Hz}$ in den beiden folgenden Grafiken vergleichend gegenübergestellt. Es handelt sich um gemittelte Dehnungsverläufe der jeweiligen Beanspruchungsgruppe, um den Einfluss von Streuungen zu reduzieren.

Die gestrichelt dargestellten Dehnungsverläufe repräsentieren die gemessenen, einhüllenden Dehnungsverläufe auf dem Oberspannungsniveau $\varepsilon_{o}$ und dem Unterspannungsniveau $\varepsilon_{u}$. Diese wurden um die Temperaturdehnungen $\varepsilon_{t}$ korrigiert. Dies führt zu einer Vergrößerung der Dehnungen. Bei den $1 \mathrm{~Hz}-$ Versuchen hat die Temperaturdehnung einen nur geringen Anteil an den Gesamtdehnungen. Die maximale Temperaturdehnung wird bei einer bezogenen Lastwechselzahl von $N / N_{f} \approx 0,2$ erreicht und nimmt bis Versuchsende nicht weiter zu. Demgegenüber liegt die Temperaturdehnung bei den $10 \mathrm{~Hz}$-Versuchen zu Versuchsende in der Größenordnung von rd. $11 \%$ der gemessenen Gesamtdehnung. Dabei wachsen die Temperaturdehnungen über die Versuchsdauer kontinuierlich an. Es wird kein thermischer Gleichgewichtszustand erreicht.

Die $1 \mathrm{~Hz}-$ Versuche dauerten im Mittel etwa 30 Stunden, dementsprechend hoch ist der viskose Dehnungsanteil $\varepsilon_{c r}$. Der Dehnungsanteil liegt bei ca. $11 \%$ der gemessenen Gesamtdehnung. Im Gegensatz dazu dauerten die $10 \mathrm{~Hz}$-Versuche nur 1 Stunde. Der viskose Dehnungsanteil ist folglich geringer und liegt bei etwa $4 \%$ der gemessenen Gesamtdehnung. 
Zwischen den viskosen Dehnungsverläufen $\varepsilon_{c r}$ und den temperaturkompensierten Dehnungsverläufen auf dem Entlastungsniveau $\varepsilon_{0 k N}-\varepsilon_{t}$ stellen sich die schädigungsinduzierten Dehnungen $\varepsilon_{d}$ dar. Die Größe dieses Dehnungsanteils ist vor allem von der Bruchlastwechselzahl abhängig. Die $1 \mathrm{~Hz}-$ Versuche weisen etwa dreimal so große Bruchlastwechselzahlen auf wie die 10 Hz-Versuche. Diese Differenz schlägt sich in den schädigungsinduzierten Dehnungsanteilen nieder. Der elastische Dehnungsanteil liegt bei beiden Versuchsreihen in derselben Größenordnung.

Die gemittelten, anteiligen Dehnungsverläufe der übrigen Beanspruchungsgruppen sind im Anhang A.4 dargestellt. Das beschriebene Bild der Dehnungsentwicklung stellt sich für die übrigen Beanspruchungsgruppen in gleicher Weise dar.

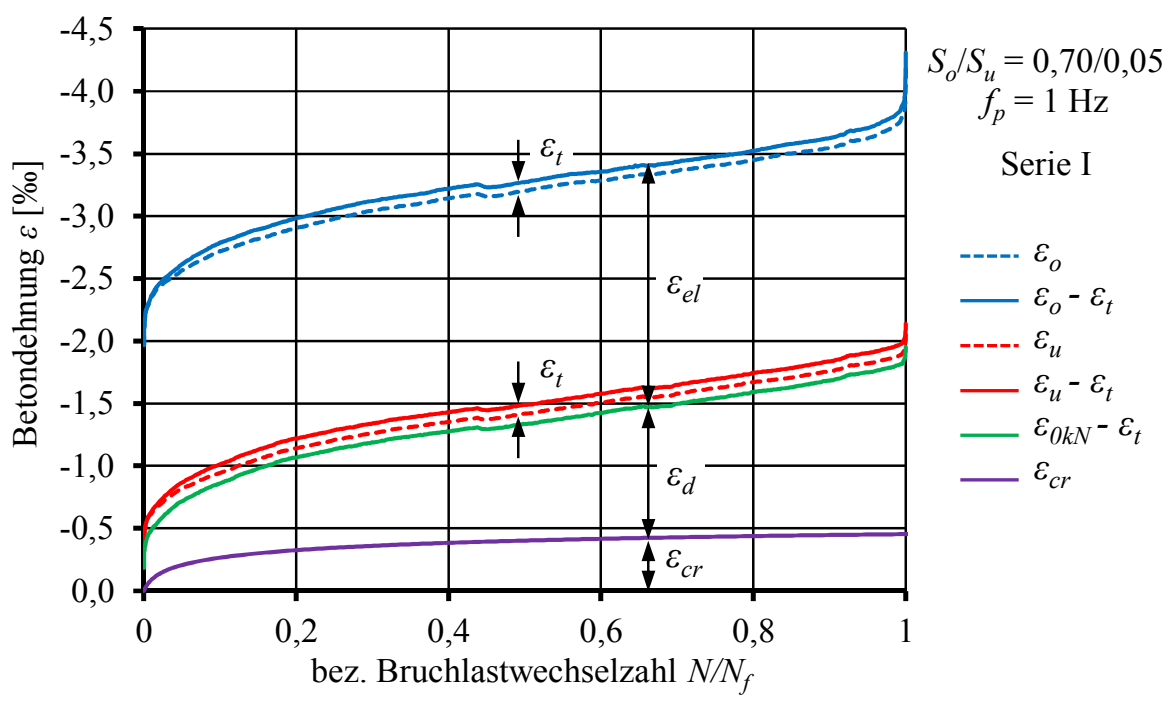

Abbildung 6.15: Gemittelte Dehnungsanteile für die Ermüdungsversuche der Serie I mit $S_{o}=0,70, S_{u}=0,05$ und $f_{p}=1 \mathrm{~Hz}$

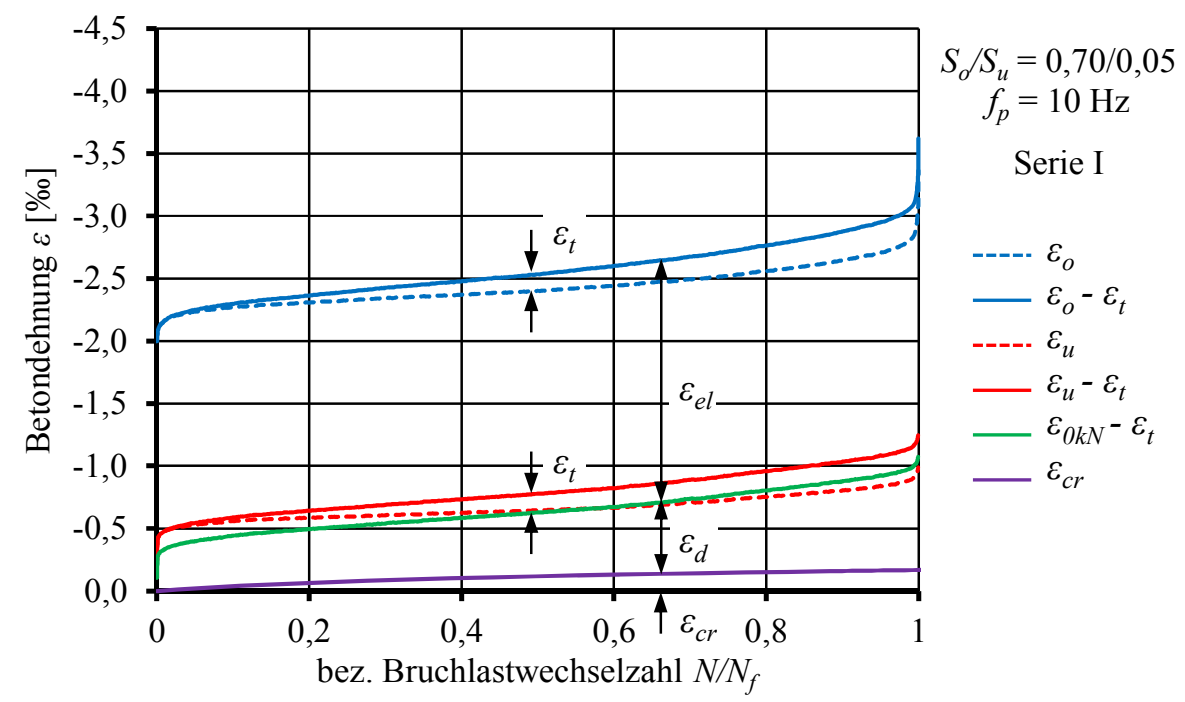

Abbildung 6.16: Gemittelte Dehnungsanteile für die Ermüdungsversuche der Serie I mit $S_{o}=0,70, S_{u}=0,05$ und $f_{p}=10 \mathrm{~Hz}$ 


\subsection{Fazit}

Die Ermüdungsbruchdehnungen der untersuchten Versuchskörper wurden entsprechend der additiven Modellvorstellung analysiert. Es erfolgte die Trennung hinsichtlich elastischer, thermischer, viskoser und schädigungsinduzierter Dehnungsanteile. Die Abbildung 6.17 stellt die prozentuale Verteilung dieser Dehnungsanteile an den gemessenen Gesamtdehnungen dar. Die Dehnungsanteile sind auf die jeweiligen, gemittelten Ermüdungsbruchdehnungen bezogen und in Abhängigkeit der untersuchten Beanspruchungsgruppen dargestellt.

Der elastische Dehnungsanteil verursacht $60 \%$ bis $80 \%$ der gemessenen Ermüdungsbruchdehnung. Er stellt damit den größten Anteil der vier Dehnungskomponenten dar. Der schädigungsinduzierte Dehnungsanteil, die nächst kleinere Gruppe, liegt in der Größenordnung von $20 \%$ bis $40 \%$ der gemessenen Ermüdungsbruchdehnung. Die Analyse des schädigungsinduzierten Dehnungsanteils zeigte, dass dieser von der logarithmierten Bruchlastwechselzahl linear abhängig ist. Der schädigungsinduzierte Dehnungsanteil spiegelt somit den linearen Verlauf der Wöhlerlinien im untersuchten Lastwechselbereich wider.

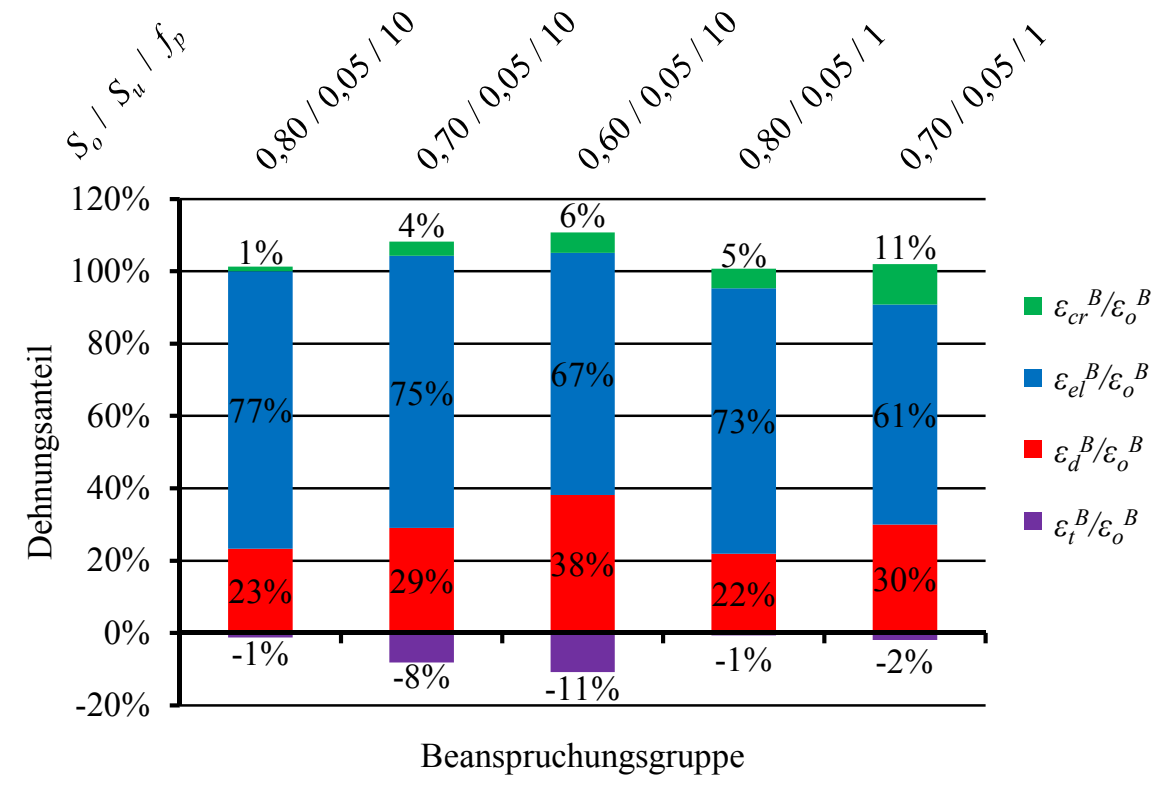

Abbildung 6.17: Prozentuale Zusammensetzung der Ermüdungsbruchdehnungen

Die Größe des viskosen Dehnungsanteils wird von der Versuchsdauer und dem kriechaffinen Beanspruchungsniveau bestimmt. Versuche mit geringeren Belastungsfrequenzen und dementsprechend längeren Versuchsdauern führen zu größeren viskosen Dehnungen als Versuche mit höheren Belastungsfrequenzen und dementsprechend kürzeren Versuchsdauern. Der Anteil der viskosen Dehnungen an den gemessenen Ermüdungsbruchdehnungen lag in den durchgeführten Versuchen bei maximal $11 \%$.

Bei den Temperaturdehnungen handelt es sich im Gegensatz zu den elastischen, schädigungsinduzierten und viskosen Dehnungsanteilen um eine Elongation des Probekörpers. Die Temperaturdehnungen verkleinern somit die gemessene Ermüdungsdehnung. Insgesamt 
können die thermischen Dehnungen einen wesentlichen Anteil an den gemessenen Gesamtdehnungen ausmachen. So erreichte der Anteil der Temperaturdehnungen bis zu 11 \% der gemessenen Ermüdungsbruchdehnungen.

Der Mittelwert und die Streubreite der Ermüdungsbruchdehnungen werden durch die thermischen und viskosen Dehnungen beeinflusst. Um diesen Zusammenhang darzustellen, werden die Ermüdungsbruchdehnungen der Versuchskörper als standardnormalverteilt angenommen. In dem nachfolgenden Diagramm werden die Verteilungsdichte (durchgezogene Linien) und die gemessenen bzw. korrigierten Ermüdungsbruchdehnungen (Punkte) dargestellt. Durch die Temperaturkompensation der Ermüdungsbruchdehnung verschiebt sich der Mittelwert in dem Diagramm nach rechts, und die Spannweite der Ermüdungsbruchdehnungen reduziert sich von 1,34 \%o auf 1,17 \%. Die Standardabweichung der Ermüdungsbruchdehnungen ändert sich jedoch nur geringfügig von $s=0,312$ auf 0,308. Erst durch die Korrektur der temperaturkompensierten Ermüdungsbruchdehnungen um die zeitabhängigen viskosen Verformungen reduzierte sich die Standardabweichung auf $s=0,253$. Auch die Spannweite der Dehnungswerte nimmt auf 0,93 \%o deutlich ab. Der Mittelwert der Ermüdungsbruchdehnung verschiebt sich in dem Diagramm wieder nach links.

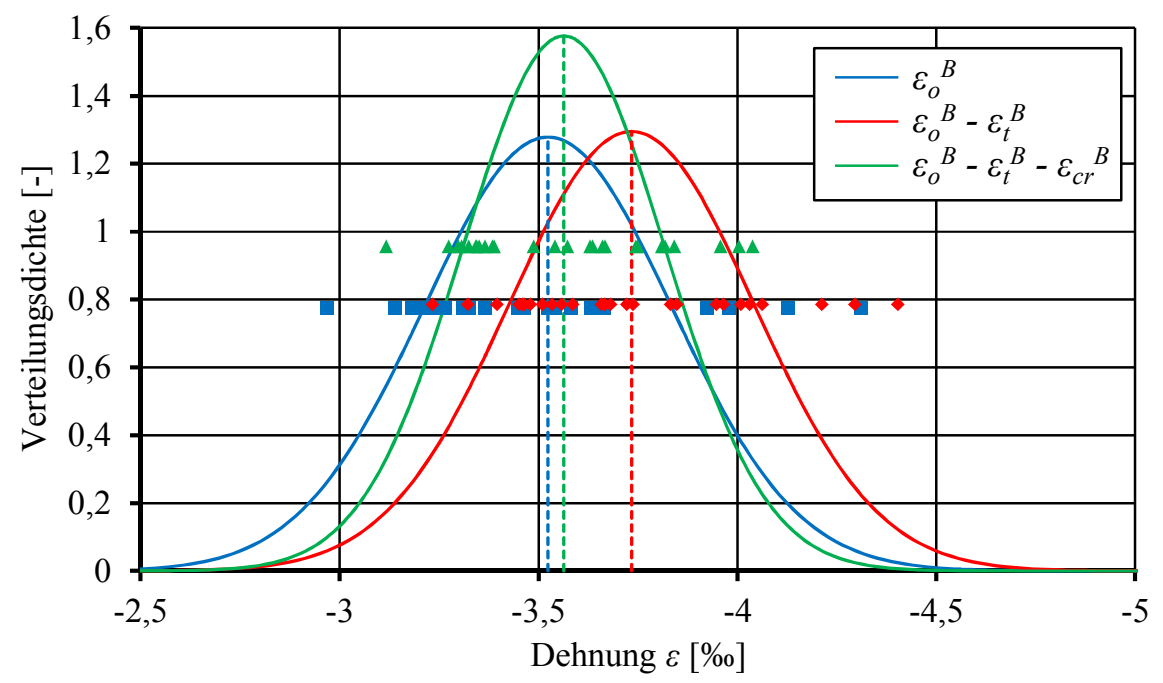

Abbildung 6.18: Verteilungsdichte der Ermüdungsbruchdehnungen

Nach der Korrektur der Ermüdungsbruchdehnungen um die thermischen und viskosen Dehnungen liegt dessen Mittelwert geringfügig oberhalb des Mittelwertes der Bruchdehnung unter monoton steigender Beanspruchung. Ob die Werte grundsätzlich miteinander korrelieren, was naheliegend ist, kann auf Grundlage der durchgeführten Versuche aber nicht abschließend beurteilt werden. Hierzu sind weitere Untersuchungen an unterschiedlichen Betonen erforderlich. Dabei sind die aufzuzeichnenden Dehnungen um die auftretenden thermischen und viskosen Dehnungsanteile entsprechend der additiven Modellvorstellung zu korrigieren. Zur Trennung bzw. Bestimmung der einzelnen Dehnungsanteile ist im Anhang A.5 eine Handlungsanweisung geben, die den notwendigen experimentellen Untersuchungsumfang und das Vorgehen umfasst. 


\section{$7 \quad$ Zusammenfassung und Ausblick}

\subsection{Zusammenfassung}

Betonkonstruktionen weisen ein höchst komplexes Ermüdungsverhalten auf. $\mathrm{Zu}$ dessen Beschreibung reicht die alleinige Betrachtung von Bruchlastwechselzahlen nicht aus, da der Beton unter zyklischen Lasten ein ausgeprägtes Verformungsverhalten aufweist. Dieses Verformungsverhalten beeinflusst die Spannungsverteilung auf Querschnittsebene und vermag sich positiv auf das Tragverhalten der Struktur auszuwirken. Derzeit gibt es kein verifiziertes Modell, das für eine Abschätzung der Verformungen herangezogen werden könnte. Es gibt Modellvorstellungen, die die Ermüdungsbruchdehnung des Betons bei der Bruchdehnung unter monoton steigender Beanspruchung, auf dem abfallenden Ast der Envelope-Kurve oder drüber hinaus annehmen. Für alle Modelle gibt es Versuche, die diese belegen oder auch widerlegen. Dabei wird aber vernachlässigt, dass die Bruchdehnung sowie die Envelope-Kurve unter monoton steigender Beanspruchung von der Belastungsgeschwindigkeit signifikant abhängen. Zudem erwärmen sich die Probekörper während des Ermüdungsversuchs merklich. Hierdurch werden Temperaturdehnungen hervorgerufen, die bei der Gegenüberstellung von monotonen und zyklischen Bruchdehnungen berücksichtigt werden müssen. Zudem treten bei den Betondehnungen im Ermüdungsversuch auch zeitabhängige, viskose Dehnungsanteile auf.

Diese Arbeit befasst sich mit dem Verformungsverhalten von Beton unter Ermüdungsbeanspruchung. Es wurde ein additives Dehnungsmodell entwickelt, in dem sich die Ermüdungsdehnungen aus elastischen, viskosen, thermischen und schädigungsinduzierten Dehnungen zusammensetzen. Die einzelnen Dehnungsanteile entwickeln sich in Abhängigkeit von der Zeit und/oder der Lastwechselzahl und werden durch die Charakteristik des Beanspruchungsverlaufs und den Materialeigenschaften des Betons unterschiedlich beeinflusst. Dabei stand die Quantifizierung des viskosen Dehnungsanteils im Mittelpunkt der Betrachtung. Das viskose Dehnungsverhalten von Beton wird gewöhnlich in Kriechversuchen untersucht. Der Kriechversuch ist durch eine konstante Druckbeanspruchung gekennzeichnet. Demgegenüber ist der Ermüdungsversuch durch das Ober- und Unterspannungsniveau sowie durch die Belastungsfrequenz charakterisiert. Es war somit eine Modellvorstellung zu entwickeln, um auf Grundlage des zyklischen Beanspruchungsverlaufs dasjenige Spannungsniveau zu ermitteln, unter dem im Kriechversuch die gleichen viskosen Verformungen auftreten wie im Ermüdungsversuch bei gleicher Versuchsdauer. Dieses Spannungsniveau wird als kriechaffines Spannungsniveau bezeichnet.

Ausgangspukt für die Bestimmung des kriechaffinen Beanspruchungsniveaus ist die Annahme, dass der aus dem zyklischen Beanspruchungsverlauf resultierende viskose Verformungsverlauf, aus dem Kriechverhalten des Betons unter dauerhaften Druckbeanspruchungen abgeleitet werden kann. Der zyklische Beanspruchungsverlauf wurde dementsprechend als impulsförmiger, 
stufenweiser Belastungsverlauf interpretiert, auf denen jeweils diskrete Kriechverformungen oder auch Rückkriechverformungen stattfinden. Die Bestimmung des kriechaffinen Beanspruchungsniveaus erfolgte auf Grundlage einer rheologischen Betrachtungsweise unter Ansatz verschiedener Kriechmodelle. Es wurde einerseits die Modellvorstellung des Alterns und andererseits die der linearen Viskoelastizität berücksichtigt. Diese Modellansätze beschreiben im Grundsatz sehr einfache Kriechansätze, sie stellen zugleich aber auch die obere und untere Grenze möglicher Kriechansätze dar. Auf dieser Grundlage konnte das kriechaffine Beanspruchungsniveau bestimmt werden. Bei Oberspannungsniveaus von bis $\mathrm{zu} 40 \%$ der Betondruckfestigkeit ergibt sich das kriechaffine Beanspruchungsniveau zum Mittelspannungsniveau des Ermüdungsversuchs. Bei höheren Oberspannungsniveaus führte die Überproportionalität des Kriechens zu einer Erhöhung des kriechaffinen Beanspruchungsniveaus. Es liegt damit zwischen Mittelspannungsniveau und Oberspannungsniveau des Ermüdungsversuchs. Der Zusammenhang wird in der Arbeit grafisch dargestellt und kann verschiedenen Diagrammen entnommen werden. Mithilfe des entwickelten Modells konnte nachgewiesen werden, dass die Belastungsfrequenz keinen Einfluss auf das kriechaffine Beanspruchungsniveau hat. Grundsätzlich ist die Proportionalität zwischen Kriechspannung und Kriechdehnung entscheidend für die Höhe des kriechaffinen Beanspruchungsniveaus. In dieser Arbeit wurde der Zusammenhang entsprechend Model Code 2010 zugrunde gelegt, der sich auch im Model Code 1990 und im Eurocode 2 wiederfindet. Bis $40 \%$ der Betondruckspannung wurde eine lineare Beziehung zwischen der Kriechspannung und der Kriechdehnung angenommen. Bei darüber hinausgehenden Kriechspannungen nehmen die Kriechdehnungen überproportional zu. Die Kriechkurve bzw. der Verlauf der Kriechfunktion hat keinen Einfluss auf das kriechaffine Beanspruchungsniveau.

Im zweiten Teil der Arbeit erfolgten experimentelle Untersuchungen an zylindrischen Betonproben. Das Verformungsverhalten des Betons unter monoton steigender, dauerhafter und zyklischer Belastung wurde ermittelt. Auf dieser Grundlage wurden die im Ermüdungsversuch auftretenden elastischen, viskosen, thermischen und schädigungsinduzierten Dehnungsanteile bestimmt und analysiert. Die thermischen und viskosen Dehnungen lagen jeweils in der Größenordnung von bis zu $11 \%$ der gemessenen Gesamtverformungen. Die Größe dieser Dehnungsanteile ist vom Belastungsszenario stark abhängig. Niedrigere Beanspruchungsniveaus und Belastungsfrequenzen führen zu längeren Versuchslaufzeiten und so zu größeren viskosen Verformungen. Im Gegenzug führen höhere Belastungsfrequenzen zu einer stärkeren Probekörpererwärmung und dementsprechend zu größeren Temperaturdehnungen sowie durch die kürzere Versuchsdauer zu kleineren viskosen Dehnungen. Durch die modellbasierte Trennung der einzelnen Dehnungsanteile konnte erstmals der schädigungsinduzierte Dehnungsanteil während eines Ermüdungsversuchs separiert werden. Es konnte festgestellt werden, dass der schädigungsinduzierte Dehnungsanteil von der logarithmierten Bruchlastwechselzahl linear abhängig ist. Damit spiegelt sich für den untersuchten Lastwechselbereich der lineare Verlauf der Wöhlerlinien, in der logarithmierten Darstellung, in dem schädigungsinduzierten Dehnungsanteil wider. 
Es hat sich bestätigt, dass sich die Dehnungen im Ermüdungsversuch aus den Anteilen elastisch, schädigungsinduziert, viskos und thermisch zusammensetzen. Dabei werden die elastischen und schädigungsinduzierten Anteile insbesondere durch die Lastwechselzahl beeinflusst, der viskose Anteil durch die Versuchsdauer und der thermische Anteil durch die Belastungsfrequenz. Darüber hinaus werden die Anteile vom Beanspruchungsniveau und von der Spannungsamplitude beeinflusst. Deren Wirkungen sind aber für alle Dehnungsanteile gleich. So führen höhere Spannungsniveaus und größere Amplituden zu einer stärkeren Materialdegradation, zu einer schnelleren schädigungsinduzierten Verformungsakkumulation und zu größeren viskosen und thermischen Dehnungen.

Die Untersuchungen zeigen, dass die „scheinbaren“ Streuungen der Ermüdungsbruchdehnungen im Wesentlichen aus den thermischen und viskosen Dehnungsanteilen des Betons resultieren. Durch die Korrektur der Ermüdungsbruchdehnungen um diese Anteile wird es möglich, die Aussagequalität der Versuchsergebnisse zu verbessern. Dies gilt insbesondere für Ermüdungsversuche, die auf unterschiedlichen Beanspruchungsniveaus und bei variierenden Belastungsfrequenzen durchgeführt werden. Die Handlungsanweisung im Anhang A.5 stellt den erforderlichen experimentellen Untersuchungsumfang zur Trennung der einzelnen Dehnungsanteile dar. Auf diese Weise wird eine neuartige Auswertemethodik zum Dehnungsverhalten von ermüdungsbeanspruchtem Beton geboten.

Die Versuchsdatenauswertung zeigt, dass bei dem untersuchten Beton die Ermüdungsbruchdehnungen, korrigiert um die thermischen und viskosen Dehnungen, die Bruchdehnungen unter monoton steigender Beanspruchung über- als auch unterschreiten. Der Mittelwert der Ermüdungsbruchdehnungen ist damit geringfügig größer als der Mittelwert der monotonen Bruchdehnungen. $\mathrm{Ob}$ diese beiden Werte grundsätzlich für alle Betone miteinander korrelieren, ist naheliegend, kann auf Grundlage der durchgeführten Versuche aber nicht abschließend beurteilt werden. Hierzu sind weitere Untersuchungen an unterschiedlichen Betonfestigkeitsklassen erforderlich. Dabei sind die aufzuzeichnenden Dehnungen, wie vorgestellt, um die auftretenden thermischen und viskosen Dehnungsanteile entsprechend der additiven Modellvorstellung zu korrigieren. Die Hypothesen nach dem Envelope-Konzept und dem energetischen Ermüdungsschädigungsmodell, vgl. Abschnitt 2.6, werden durch die Versuchsergebnisse nicht bestätigt.

\subsection{Ausblick}

In der vorliegenden Arbeit wurden die Dehnungsanteile eines Betonkörpers im Ermüdungsversuch untersucht. Es hat sich gezeigt, dass die getroffenen Annahmen einen ersten Schritt in Richtung einer differenzierten Verformungsbetrachtung darstellen. Die Betrachtung des viskosen Verformungsanteils stand im Fokus der Betrachtung. Die elastischen, thermischen und schädigungsinduzierten Verformungen müssen weiter betrachtet und untersucht werden.

Die elastische Dehnungsänderung stellt sich in erster Linie als Folge der Steifigkeitsdegradation dar. Diese wird durch Rissbildungsprozesse im Beton hervorgerufen. Weitergehende 
Untersuchungen zum Einfluss des Beanspruchungsniveaus und der Belastungsfrequenz auf die resultierende Steifigkeitsdegradation wären wünschenswert. Von größerem Interesse wäre jedoch, auf Grundlage der Gefügezerrüttung bzw. Rissbildung und Rissdichte auf die Steifigkeitsdegradation zu schließen. Es gilt somit, das Gefüge des Betons während des Versuchs genauer zu untersuchen. Dabei können einerseits Verfahren wie Schallemissionsanalysen und Ultraschallverfahren helfen, aber insbesondere bildgebende Verfahren wie Röntgen oder Computertomografie, wie sie in jüngster Zeit im Betonbau möglich werden, können hier entscheidende Impulse zum tieferen Verständnis liefern.

Der Ursprung für die Wärmefreisetzung im ermüdungsbeanspruchten Betonkörper wird in der Materialdämpfung und in Reibprozessen auf der Mikroebene gesehen. Die Erwärmung und die resultierenden Temperaturdehnungen sind eine Folge dessen. In Zukunft sollte die Wärmefreisetzung im Betonkörper genau untersucht und quantifiziert werden. Des Weiteren sind die Temperaturgradienten und die resultierenden Eigenspannungszustände sowie ihr Einfluss auf den Ermüdungswiderstand genauer zu analysieren. Zudem beeinflusst die Probeköpertemperatur auch die Festigkeit, den Elastizitätsmodul und das Kriechverhalten des Betons und somit auch dessen Dehnungsverhalten. Diese Interaktion ist im Rahmen der additiven Modellvorstellung zunächst vernachlässigt worden, stellt aber eine erforderliche Weiterentwicklung des Modells dar.

Es zeigte sich, dass der schädigungsinduzierte Dehnungsanteil von der logarithmierten Bruchlastwechselzahl linear abhängig ist. Diese Beobachtung ist im Rahmen von weiteren Untersuchungen zu überprüfen. Es stellt sich auch die Frage, ob die schädigungsinduzierten Dehnungen auf Grundlage von bildgebenden Verfahren zur Darstellung von Gefügeveränderungen visualisiert werden können. Damit könnten aufschlussreiche, weiterführende Informationen generiert werden. Ebenso stellt die Abbildung des schädigungsinduzierten Dehnungsanteils mithilfe rheologischer Grundelemente eine interessante Weiterentwicklung der additiven Modellvorstellung dar.

Die viskosen Verformungen sind im Rahmen dieser Arbeit als Summe aus irreversiblen und reversiblen Dehnungen betrachtet worden. Die Trennung dieser Anteile bedarf der Durchführung von Ermüdungsversuchen, die nicht bis zum Bruch belastet werden, sondern nach einer bestimmten Versuchsdauer beendet werden, um das Relaxationsverhalten des Betons zu erfassen. Auf dieser Basis können dann die reversiblen Anteile erfasst und analysiert werden um weiterführende Informationen zum viskosen Verformungsverhalten von ermüdungsbeanspruchtem Beton zu erhalten.

Der Anwendungshorizont des entwickelten additiven Dehnungsmodells erstreckt sich in dieser Arbeit auf den Ermüdungsversuch. Die Übertragung des Modells auf das reale Bauteil bietet die Möglichkeit das Tragverhalten bei Ermüdungsbeanspruchung genauer abschätzen zu können als mit dem derzeitigen, normativen Nachweiskonzept auf Grundlage von Bruchlastwechselzahlen. 


\section{Literaturverzeichnis}

Anders, I. (2012): Stoffgesetz zur Beschreibung des Kriech- und Relaxationsverhaltens junger normal- und hochfester Betone. Dissertation, Karlsruher Institut für Technologie (KIT).

Awad, M. E; Hilsdorf, H. K. (1971): Strength and Deformation Characteristics of Plain Concrete Subjected to High Repeated and Sustained Loads. University of Illinois.

Bahn, B. Y; Hsu, C.-T. T. (1998): Stress-Strain Behavior of Concrete under Cyclic Loading. ACI Material Journal 95 (1998) Heft 2, S. 178-193.

Ban, S. (1933): Der Ermüdungsvorgang von Beton. Der Bauingenieur (1933) Heft 13/14, S. 188192.

Bazant, Z. P. (1968): Langzeitliche Durchbiegung von Spannbetonbrücken infolge des Schwingkriechens unter Verkehrslasten. Beton- und Stahlbetonbau 12 (1968), S. 282-285.

Bazant, Z. P; Hauggaard, A. B; Baweja, S; Ulm, F.-J. (1997): Microprestress-Solidification Therory for Concrete Creep. I: Aging and Drying Effects II: Algorithm and Verification. Journal of Engineering Mechanics 123 (1997), S. 1188-1201.

Bazant, Z. P; Kim, J.-K. (1992): Improved Prediction Model for Time-Dependent Deformations of Concrete. Part 5 - Cyclic Load and Cyclic Humidity. Materials and Structures 25 (1992), S. $163-169$.

Bazant, Z. P; Osman, E. (1976): Double Power Law for Basic Creep of Concrete. Materials and Structures 49 (1976) Heft 9, S. 3-11.

Bazant, Z. P; Prasannan, S. (1989): Solidification Theory for Concrete Creep. I: Formulation II: Verification and Application. Journal of Engineering Mechanics 115 (1989) Heft 8, S. $1691-1725$.

Bockhold, J; Stangenberg, F. (2004): Modellierung des nichtlinearen Kriechens von Beton. Beton- und Stahlbetonbau 99 (2004) Heft 3, S. 209-216.

Boes, A. (1971): Über das Kriechen von Zementstein, Mörtel und Beton. Dissertation, RheinischWestfälischen Technischen Hochschule (RWTH) Aachen.

Brecht, J; Eisenblätter, J; Jax, P. (1973): Werkstoffprüfung mit der Schallemissionsanalyse (SEA). Zeitschrift für Werkstofftechnik 4 (1973) Heft 6, S. 306-314.

Breitenbücher, R; Ibuk, H; Osterminski, K. (2007): Veränderung der Steifigkeit und des Dehnungsverhaltens von Normalbeton bei zyklischer Druckschwellbeanspruchung mit Ruhephasen. Beton- und Stahlbetonbau 102 (2007) Heft 2, S. 80-87.

Breitenbücher, R; Ibuk, H; Yüceoglu, S. (2008): Beeinflusst die Kornsteifigkeit der Gesteinskörnung im Beton den Degradationsprozess infolge zyklischer Druckbeanspruchung? Beton- und Stahlbetonbau 103 (2008) Heft 5, S. 318-323.

CEB (1988): Fatigue of Concrete Structures - State of the Art Report. Comité Euro - International du Béton, Bulletin D’Information No. 188, Lausanne, Schweiz. 
CEB (1990): Evaluation of the Time Dependent Behavior of Concrete. Comite Euro International du Béton, Bulletin D’Information No. 199, Lausanne, Schweiz.

DAfStb (2012): Erläuterungen zu DIN EN 1992-1-1 und DIN EN 1992-1-1/NA (Eurocode 2). Deutscher Ausschuss für Stahlbeton, Heft 600.

DAfStb (1991): Prüfung von Beton. Empfehlungen und Hinweise als Ergänzung zu DIN 1048. Deutscher Ausschuss für Stahlbeton (DAfStb), Heft 422.

DIN 1048-5 (1991): Prüfverfahren für Beton - Festbeton, gesondert hergestellte Probekörper. Deutsches Institut für Normung e.V., Beuth Verlag.

DIN 50100 (1978): Dauerschwingversuch. Deutsches Institut für Normung e.V.

DIN EN 12390-3 (2009): Prüfung von Beton - Teil 3: Druckfestigkeit von Prüfkörpern. Deutsches Institut für Normung e.V., Beuth Verlag.

DIN EN 12390-13 (2014): Prüfung von Beton - Teil 13: Bestimmung des Elastizitätsmoduls unter Druckbelastung (Sekantenmodul). Deutsches Institut für Normung e.V., Beuth Verlag.

Dischinger, F. (1937): Verformung und Kriechen des Betons bei Bogenbrücken. Bauingenieur 18 (1937) Heft 33/34, 35/36, 39/40, S. 487-520, 539-552, 595-621.

Do, M. T; Chaallal, O; Aitcin, P. C. (1993): Fatigue Behavior of High-Performance Concrete. Journal of Material Civil Engineering 5 (1993) Heft 1, S. 96-111.

Elsmeier, K. (2015): Influence of Temperature on the Fatigue Behaviour of concrete. In: fib Symposium, 18.-20. Mai 2015, Kopenhagen, Dänemark.

Elsmeier, K; Hümme, J; Oneschkow, N; Lohaus, L. (2016): Prüftechnische Einflüsse auf das Ermüdungsverhalten hochfester feinkörniger Vergussbetone. Beton- und Stahlbetonbau 111 (2016) Heft 4, S. 233-240.

Elsmeier, K; Lohaus, L. (2014): Temperature Development of Concrete due to Fatigue Loading. In: Proc. of the $10^{\text {th }}$ fib International PhD Symposium in Civil Engineering, 21.-23. Juli 2014, Québec, Kanada, S. 137-142.

Eurocode 2: Bemessung und Konstruktion von Stahlbeton- und Spannbetontragwerken: DIN EN 1992-1-1 (2011): Allgemeine Bemessungsregeln und Regeln für den Hochbau. DIN EN 1992-2 (2010): Betonbrücken. Deutsches Institut für Normung e.V., Beuth Verlag.

Finck, F. (2005): Untersuchungen von Bruchprozessen in Beton mit Hilfe der Schallemissionsanalyse. Dissertation, Universität Stuttgart.

Fischer, L. (1999): Sicherheitskonzept für neue Normen - ENV und DIN-neu. Grundlagen und Hintergrundinformationen Teil 3: Statistische Auswertung von Stichproben im eindimensionalen Fall. Bautechnik 76 (1999) Heft 2, S. 167-179.

Freudenthal, A. M. (1955): Inelastisches Verhalten von Werkstoffen. VEB Verlag Technik, Berlin.

Gaede, K. (1962): Versuche über die Festigkeit und Verformung von Beton bei Druckschwellbeanspruchung. Deutscher Ausschuss für Stahlbeton (DAfStb), Heft 144. 
Göhlmann, J. (2009): Zur Schädigungsberechnung an Betonkonstruktionen für Windenergieanlagen unter mehrstufiger und mehraxialer Ermüdungsbeanspruchung. Dissertation, Leibniz Universität Hannover.

Grünberg, J; Göhlmann, J. (2013): Concrete Structures for Wind Turbines. Ernst \& Sohn.

Hanson, J. A. (1953): A Ten-Year Study of Creep Properties of Concrete, Concrete Laboratory Report Sp-38.

Hilsdorf, H. K; Müller, H. S. (1987): Stoffgesetze für das Kriechen und Schwinden von Dischinger bis heute. In: Specht, M. (Hrsg.): Spannweite der Gedanken - Zur 100. Wiederkehr des Geburtstages von Franz Dischinger. Springer, Berlin, Heidelberg, S. 193212.

Holmen, J. O. (1979): Fatigue of Concrete by Constant and Variable Amplitude Loading. Dissertation, Universität von Trondheim.

Hsu, T. T. C. (1981): Fatigue of Plain Concrete. ACI Journal 78 (1981) Heft 27, S. 292-305.

Ibuk, H. (2008): Ermüdungsverhalten von Beton unter Druckschwellbelastung. Dissertation, Ruhr-Universität Bochum.

Jinawath, P. (1974): Cumulative Fatigue Damage of Plain Concrete in Compression. Dissertation, University of Leeds.

Karsan, I. D; Jirsa, J. O. (1969): Behavior of Concrete under Compressive Loadings. Journal of Structural Division (ASCE) 95 (1969) Heft 12, S. 2543-2563.

Klausen, D; Weigler, H. (1979): Betonfestigkeit bei konstanter und veränderlicher Dauerschwellbeanspruchung. Betonwerk + Fertigteil-Technik (1979) Heft 3, S. 158-163.

König, G; Danielewicz, I. (1994): Ermüdungsfestigkeit von Stahlbeton- und Spannbetonbauteilen mit Erläuterungen zu den Nachweisen gemäß CEB-FIP Model Code 1990. Deutscher Ausschuss für Stahlbeton (DAfStb), Heft 439.

Kono, S; Hasegawa, H; Mori, K; Ichioka, Y., et al. (2008): Low Cycle Fatigue Characteristics of High Strength Concrete. In: $8^{\text {th }}$ International Symposium on utilization of high-strength and high-performance concrete, S. 616-621.

Krümmeling, S. (1999): Schallemissionsanalyse zum Nachweis der Materialermüdung im Baustahl. Dissertation, Technische Universität Carola-Wilhelmina zu Braunschweig.

Kuryllo, A; Kwascha, W; Lewschitsch, W. (1976): Versuche über das Verhalten auf Biegung beanspruchter Stahlbeton-Bauteile unter häufig wiederholter Belastung. Beton- und Stahlbetonbau (1976) Heft 4, S. 103-106.

Lorman, W. R. (1940): The Theory of Concrete Creep. Proceedings of ASTM 40 (1940), S. $1082-1102$.

Maher, A; Darwin, D. (1982): Mortar Constituent of Concrete in Compression. ACI Journal 79 (1982) Heft 2, S. 100-109.

Markeset, G. (1993): Failure of Concrete under Compressive Strain Gradients. Dissertation, Universität von Trondheim. 
Marx, S; Grünberg, J; Hansen, M; Schneider, S. (2013): Über den Stand der Forschung zu Grenzzuständen der Ermüdung von dynamisch hoch beanspruchten Tragwerken aus Beton. Abschlussbericht zum DAfStb-Forschungsvorhaben V474, Institut für Massivbau, Leibniz Universität Hannover.

Mehlhorn, G; Kollegger, J. (1995): Anwendung der Finite Element Methode im Stahlbetonbau. In: Mehlhorn, G. (Hrsg.): Der Ingenieurbau. Rechnerorientierte Baumechanik. Ernst \& Sohn, S. 293-425.

Mehmel, A; Kern, E. (1962): Elastische und plastische Stauchungen von Beton infolge Druckschwell- und Standbelastung. Deutscher Ausschuss für Stahlbeton (DAfStb), Heft 153.

Miner, M. A. (1945): Cumulative Damage in Fatigue. Journal of Applied Mechanics (1945) Heft Nr. 12, S. A159-A164.

Model Code 1990: CEB-fib Model Code 1990 - Design Code. Comité Euro - International du Béton, 1993, Thomas Telford Services Ltd.

Model Code 2010: fib Model Code for Concrete Structures 2010. Fédération International du Béton, 2013, Ernst \& Sohn.

Müller, H. S. (1986): Zur Vorhersage des Kriechens von Konstruktionsbeton. Dissertation, Universität Fridericiana zu Karlsruhe.

Newmann, K. (1965): Criteria for the Behaviour of Plain Concrete under Complex States of Stress. Proceedings of an International Conference on the Structure of Concrete. September 1965, London, Großbritannien.

Ondracek, G. (1992): Werkstoffkunde. Leitfaden für Studium und Praxis. Expert Verlag.

Oneschkow, N. (2016): Fatigue Behaviour of High-Strength Concrete with Respect to Strain and Stiffness. International Journal of Fatigue 87 (2016), S. 38-49.

Oneschkow, N. (2014): Analyse des Ermüdungsverhaltens von Beton anhand der Dehnungsentwicklung. Dissertation, Leibniz Universität Hannover.

Palmgren, A. (1924): Die Lebensdauer von Kugellagern. Zeitschrift des Vereins Deutscher Ingenieure 68 (1924) Heft 14, S. 339-341.

Papa, E. (1993): A Damage Model for Concrete Subjected to Fatigue Loading. European Journal of Mechanics - A/Solids 12 (1993) Heft 3, S. 429-440.

Park, Y. J. (1990): Fatigue of Concrete Under Random Loadings. Journal of Structural Engineering (ASCE) 116 (1990), S. 3228-3235.

Petkovic, G; Rosseland, S; Stemland, H; Lenschow, R. (1992): High Strength Concrete SP3 Fatigue. Report 3.2: Fatigue of High Strength Concrete. SINTEF Structural Engineering FCB.

Pfanner, D. (2003): Zur Degradation von Stahlbetonbauteilen unter Ermüdungsbeanspruchung. Dissertation, Ruhr-Universität Bochum.

Pfefferle, R. (1971): Zur Theorie des Betonkriechens. Dissertation, Universität Karlsruhe.

Pfister, T. R. (2007): Ein Ermüdungsschädigungsmodell für Zuverlässigkeitsanalysen von Stahlbetontragwerken. Dissertation, Ruhr-Universität Bochum. 
Plähn, J. (1969): Zur Frage der bleibenden Verformungen des Betons bei Dauerschwingbeanspruchung. Beton- und Stahlbetonbau 64 (1969) Heft 12, S. 292-295.

Pölling, R. (2000): Eine praxisnahe, schädigungsorientierte Materialbeschreibung von Stahlbeton für Strukturanalysen. Dissertation, Ruhr-Universität Bochum.

Rasch, C. (1962): Spannungs-Dehnungs-Linien des Betons und Spannungsverteilung in der Biegedruckzone bei konstanter Dehngeschwindigkeit. Deutscher Ausschuss für Stahlbeton (DAfStb), Heft 154.

Rehm, G; Diem, P; Zimbelmann, R. (1977): Technische Möglichkeiten zur Erhöhung der Zugfestigkeit von Beton. Deutscher Ausschuss für Stahlbeton (DAfStb), Heft 283.

Reinhardt, H. W; Stroeven, P; den Uijl, J. A; Kooistra, T. R; Vrencken, J. H. (1978): Einfluss von Schwingbreite, Belastungshöhe und Frequenz auf die Schwingfestigkeit von Beton bei niedrigen Bruchlastwechselzahlen. Betonwerk + Fertigteil-Technik (1978) Heft 9, S. 498503.

Reinhardt, H.-W; Hilsdorf, H. K. (2001): Beton. In: Eibl, J. (Hrsg.): Betonkalender 2001 Teil 1. Ernst \& Sohn, S. 1-144.

Ritter, R. (2014): Verformungsverhalten und Grenzflächen von Ultrahochleistungsbeton unter mehraxialer Beanspruchung. Dissertation, Technische Universität Dresden.

Ross, A. D. (1937): Concrete Creep Data. The Structural Engineer 15 (1937) Heft 8, S. 314-326.

Rostásy, F. S; Alda, W; Teichen, K.-T. (1975): Kriechen und Schwingkriechen von Leichtbeton. Bauingenieur 50 (1975) Heft 12, S. 455-459.

Rüsch, H. (1960): Researches Towards a General Flexural Theory for Structural Concrete. Journal of the American Concrete Institute 57 (1960) Heft 1, S. 1-28.

Schläfli, M. (1999): Ermüdung von Brückenfahrbahnplatten aus Stahlbeton. Dissertation, Ecole Polytechnique Fédérale de Lausanne.

Schneider, S; Vöcker, D; Marx, S. (2012): Zum Einfluss der Belastungsfrequenz und der Spannungsgeschwindigkeit auf die Ermüdungsfestigkeit von Beton. Beton- und Stahlbetonbau 107 (2012) Heft 12, S. 836-845.

Schwabach, E. (2005): Verformungs- und Degradationsverhalten von niederzyklisch uniaxial druckbeanspruchtem Beton. Dissertation, Bauhaus-Universität Weimar.

Shah, S. P; Fafitis, A; Arnold, R. (1983): Cyclic Loading of Spirally Reinforced Concrete. Journal of Structural Engineering 109 (1983) Heft 109, S. 1695-1710.

Shank, J. R. (1935): The Plastic Flow of Concrete. Ohio State University Exp. Sta., Bull. 91.

Shen, J.-H. (1992): Lineare und nichtlineare Theorie des Kriechens und der Relaxation von Beton unter Druckbeanspruchung. Deutscher Ausschuss für Stahlbeton (DAfStb), Heft 432.

Siebel, E. (1989): Verformungsverhalten, Energieaufnahme und Tragfähigkeit von Normal- und Leichtbeton im Kurzzeitdruckversuch. Forschungsinstitut der Zementindustrie, Heft 50/1989.

Sinha, B. P; Gerstle, K. H; Tulin, L. G. (1964): Stress-Strain Relations for Concrete under Cyclic Loading. Journal of the American Concrete Institute (1964) Heft 2, S. 195-211. 
Smadi, M. M; Slate, F. O. (1989): Microcracking of High and Normal Strength Concretes under Short- and Long-Term Loadings. ACI Material Journal 86 (1989) Heft 2, S. 117-127.

Sparks, P. R; Menzies, J. B. (1973): The Effect of Rate of Loading upon the Static and Fatigue Strength of Plain Concrete in Compression. Magazine of Concrete Research 25 (1973) Heft 83, S. 73-80.

Spooner, D. C; Dougill, J. W. (1975): A Quantitative Assessment of Damage Sustained in Concrete during Compressive Loading. Magazine of Concrete Research 27 (1975) Heft 92, S. 151-160.

Spooner, D. C; Pomeroy, C. D; Dougill, J. W. (1976): Damage and Energy Dissipation in Cement Pasts in Compression. Magazine of Concrete Research 28 (1976) Heft 94, S. 21-29.

Straub, L. G. (1931): Plastic Flow in Concrete Arches. American Society of Civil Engineers 95 (1931).

Streit, W. (1991): Zeitabhängige Verformungen von Beton infolge hoher Druckdauerbeanspruchung. Dissertation, Technische Universität München.

Teichen, K.-T. (1968): Über die innere Dämpfung von Beton. Dissertation, Universität Stuttgart.

Thiele, M; Pirskawetz, S; Baeßler, M; Rogge, A. (2011): Untersuchung der Schädigungsevolution in Beton unter Ermüdungsbeanspruchung mit Hilfe der Schallemissionsanalyse. 18. Kolloquium Schallemission, Deutsche Gesellschaft für Zerstörungsfreie Prüfung e.V. (DGZfP).

Tue, N. V; Mucha, S. (2006): Ermüdungsfestigkeit von hochfestem Beton unter Druckbeanspruchung. Bautechnik 83 (2006) Heft 7, S. 497-504.

Urban, S; Wagner, R; Strauss, A; Dallinger, S., et al. (2014): Monitoring des realen Grades der Ermüdungsschädigung in Betonstrukturen. Beton- und Stahlbetonbau 109 (2014) Heft 7, S. $473-485$.

van Leeuwen, J; Siemes, A. J. M. (1979): Miner's Rule with Respect to Plain Concrete. Heron 24 (1979) Heft 1, S. 1-34.

van Mier, J. G. M; Reinhardt, H. W; van der Vlugt, B. W. (1987): Ergebnisse dreiachsiger verformungsgesteuerter Belastungsversuche an Beton. Bauingenieur 62 (1987) Heft 8, S. $353-361$.

von der Haar, C; Hümme, J; Marx, S; Lohaus, L. (2015a): Untersuchungen zum Ermüdungsverhalten eines höherfesten Normalbetons. Beton- und Stahlbetonbau 110 (2015) Heft 10, S. 699-709.

von der Haar, C; Marx, S; Krompholz, R. (2015b): Ultraschalluntersuchungen an statisch beanspruchten Betonproben. Beton- und Stahlbetonbau 110 (2015) Heft 11, S. 759-766.

von der Haar, C; Marx, S. (2016a): Development of Stiffness and Ultrasonic Pulse Velocity of Fatigue Loaded Concrete. Structural Concrete 17 (2016) Heft 4.

von der Haar, C; Marx, S. (2016b): Untersuchungen zur Steifigkeit und Ultraschallgeschwindigkeit dynamisch beanspruchter Betonproben. Beton- und Stahlbetonbau 111 (2016) Heft 3, S. 141-148. 
von der Haar, C.; Wedel, F.; Marx, S. (2016): Numerical and Experimental Investigations of the Warming of Fatigue-Loaded Concrete. In: fib Symposium 2016, Kapstadt, Süd Afrika, 21.-23. November 2016.

Wefer, M. (2010): Materialverhalten und Bemessungswerte von ultrahochfestem Beton unter einaxialer Ermüdungsbeanspruchung. Dissertation, Leibniz Universität Hannover.

Whaley, C. P; Neville, A. M. (1973): Non-elastic deformation of concrete under cyclic compression. Magazine of Concrete Research 25 (1973) Heft 84, S. 145-154.

Wischers, G. (1978a): Aufnahme und Auswirkungen von Druckbeanspruchung auf Beton. Beton (1978) Heft 2, S. 63-67.

Wischers, G. (1978b): Aufnahme und Auswirkungen von Druckbeanspruchung auf Beton. Beton (1978) Heft 3, S. 98-103.

Wischers, G; Lusche, M. (1972a): Einfluss der inneren Spannungsverteilung auf das Tragverhalten von druckbeanspruchtem Normal- und Leichtbeton. Beton (1972) Heft 8, S. $343-347$.

Wischers, G; Lusche, M. (1972b): Einfluss der inneren Spannungsverteilung auf das Tragverhalten von druckbeanspruchtem Normal- und Leichtbeton. Beton (1972) Heft 9, S. $397-403$.

Wittmann, F. H. (1986): Structure of Concrete and Crack Formation. In: Herrmann, K. P., Larsson, L. H. (Hrsg.): Fracture of Non-Metallic Materials. Springer Netherlands, Dordrecht, S. 309-340.

Wriggers, P; Nackenhorst, U; Beuermann, S; Spiess, H; Löhnert, S. (2005): Technische Mechanik kompakt. Starrkörperstatik, Elastostatik, Kinetik. B. G. Teubner Verlag, Wiesbaden.

Yankelevsky, D. Z; Reinhardt, W. (1987): Model for Cyclic Compressive Behavior of Concrete. Journal of Structural Engineering (ASCE) 113 (1987) Heft 2, S. 228-240.

Zilch, K; Zehetmaier, G. (2010): Bemessung im konstruktiven Betonbau. Springer, Berlin, Heidelberg. 


\section{Abbildungsverzeichnis}

Abbildung 2.1: Betrachtungsskalen von Beton aus (Wittmann, 1986) ........................................ 7

Abbildung 2.2: Modellvorstellung zur Rissbildung aus (Zilch \& Zehetmaier, 2010) .................... 8

Abbildung 2.3: Spannungsdehnungslinie von druckbeanspruchtem Beton mit schematischer Darstellung der Rissbildung nach (Mehlhorn \& Kollegger, 1995) ........................................... 8

Abbildung 2.4: Spannungsdehnungslinie in Abhängigkeit von der Belastungsgeschwindigkeit nach (Rüsch, 1960)

Abbildung 2.5: Dauerstandgrenze und Verformungen in Abhängigkeit von der Beanspruchungsgeschwindigkeit nach (Rasch, 1962) ........................................................ 9

Abbildung 2.6: Dehnungskomponenten von Beton unter einer zeitlich begrenzten Last..... 10

Abbildung 2.7: Kriechphasen bei Dauerlasten oberhalb der Dauerstandfestigkeit nach (Streit, 1991)

Abbildung 2.8: Lineares und nichtlineares Kriechen nach (Zilch \& Zehetmaier, 2010)

Abbildung 2.9: Verformungsansätze nach (Dischinger, 1937) und (Pfefferle, 1971)

Abbildung 2.10: Poynting-Thomson-Modell (links) und Burgers-Modell (rechts)...

Abbildung 2.11: Theorie des Alterns (links) und Theorie der linearen Viskoelastizität (rechts) nach (Zilch \& Zehetmaier, 2010).

Abbildung 2.12: Horizontale (links) und vertikale Superpositionsmethode (rechts) bei einem veränderlichen Spannungsverlauf nach (Anders, 2012) .....

Abbildung 2.13: Spannungsdehnungslinie von Beton bei niederzyklischer Druckbeanspruchung nach (Karsan \& Jirsa, 1969).

Abbildung 2.14: Entwicklung des „Stability Points“ nach (Maher \& Darwin, 1982)

Abbildung 2.15: Kennwerte und Beanspruchungsbereiche von Ermüdungsversuchen in Anlehnung an (DIN 50100, 1978)

Abbildung 2.16: Wöhlerlinien für ermüdungsbeanspruchtem Beton nach Model Code 2010 ...... 26

Abbildung 2.17: Wöhlerlinien mit unterschiedlicher Versagenswahrscheinlichkeiten nach (Holmen, 1979)

Abbildung 2.18: Entwicklung der Ultraschallgeschwindigkeit in Abhängigkeit von der

Lastwechselzahl (von der Haar \& Marx, 2016b)

Abbildung 2.19: Dehnungsentwicklung mit schematischer Darstellung der Rissbildung nach (Göhlmann, 2009)

Abbildung 2.20: Parameterdefinition für den Dehnungsverlauf auf dem Oberspannungsniveau.. 28 
Abbildung 2.21: Entwicklung des Sekantenmoduls nach (Holmen, 1979)................................. 30

Abbildung 2.22: Zyklische Spannungsdehnungsbeziehung nach (Jinawath, 1974) ..................... 30

Abbildung 2.23: Dehnungsverlauf unter monoton steigender Beanspruchung und Ermüdungsbruchdehnungen bei zyklischer Beanspruchung aus Versuchen von (Awad \&

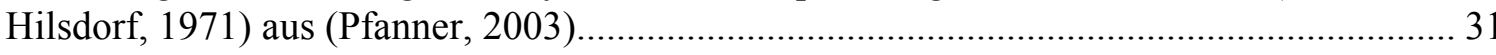

Abbildung 2.24: Bruchdehnungen und Ermüdungsbruchdehnungen aus (Holmen, 1979)............ 32

Abbildung 2.25: Ermüdungsbruchdehnung in Abhängigkeit der Zeit aus (Holmen, 1979) .......... 32

Abbildung 2.26: Temperaturbilder eines ermüdungsbeanspruchten Betonprobekörpers aus (Elsmeier, 2015)

Abbildung 2.27: Spannungsdehnungslinie und Energieverteilung eines ideal-elastischen und elasto-plastischen Werkstoffs sowie für Beton nach (Wischers, 1978a) ............................... 38

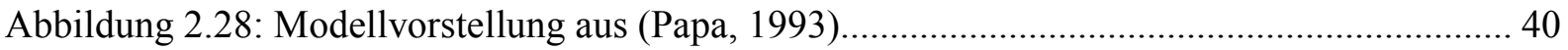

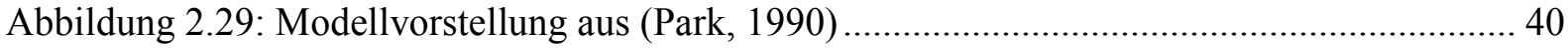

Abbildung 2.30: Spannungsdehnungslinie von (Pölling, 2000) nach (Pfanner, 2003) .................. 41

Abbildung 2.31: Definition des Ermüdungsbruchs nach (Pfanner, 2003) .................................... 42

Abbildung 3.1: Modellvorstellung zum elastischen Dehnungsanteil........................................... 45

Abbildung 3.2: Dehnungsanteile im Ermüdungsversuch .......................................................... 48

Abbildung 3.3: Messbare Probekörperdehnung im Ermüdungsversuch ....................................... 48

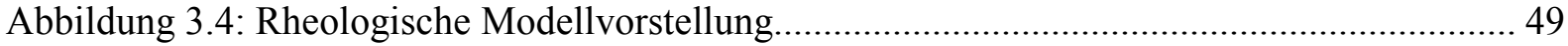

Abbildung 4.1: Modelle zur Betrachtung der viskosen Verformungen Links: Reversibles Verformungsmodell Rechts: Irreversibles Verformungsmodell .......................................... 53

Abbildung 4.2: Schaubild zur Bestimmung des kriechaffinen Beanspruchungsniveaus ............... 55

Abbildung 4.3: Superpositionsprinzip und Parameterdefinition ............................................... 57

Abbildung 4.4: Kriechverläufe nach Model Code 2010 (durchgezogene Linien) und Nachrechnung mit dem reversiblen und irreversiblen Modell (Punkte).....

Abbildung 4.5: Feder- und Dämpfereigenschaften in Abhängigkeit des Spannungsniveaus und des Betonalters

Abbildung 4.6: Verformungen des reversiblen und irreversiblen Modells unter einer zeitlich begrenzten Beanspruchung

Abbildung 4.7: Belastungsverlauf (oben) und Verformungsverlauf des reversiblen und irreversiblen Modells (unten) infolge einer periodischen, rechteckförmigen Belastung ........ 64

Abbildung 4.8: Verformungsverlauf des irreversiblen Modells im Detail.................................... 65

Abbildung 4.9: Einfluss des Spannungsniveaus auf die Kriechverformung ................................. 66 
Abbildung 4.10: Verformungsverlauf des reversiblen Modells im Detail

Abbildung 4.11: Kriechaffines Spannungsniveau in Abhängigkeit vom Mittelspannungsniveau und der Spannungsamplitude für einen rechteckförmigen, periodischen

Beanspruchungsverlauf.

Abbildung 4.12: Kriechaffines Spannungsniveau in Abhängigkeit vom Mittelspannungsniveau und der Spannungsamplitude für einen sinusförmigen Beanspruchungsverlauf.

Abbildung 4.13: Betrachteter Lastwechsel zur Bestimmung des kriechaffinen Beanspruchungsniveaus

Abbildung 4.14: Viskoser Verformungsverlauf infolge einer zyklischen Beanspruchung bei einer Belastungsfrequenz von $f_{p}=0,1 \mathrm{~Hz}$

Abbildung 4.15: Viskoser Verformungsverlauf infolge einer zyklischen Beanspruchung bei einer Belastungsfrequenz von $f_{p}=0,0028 \mathrm{~Hz}$.

Abbildung 4.16: Kriechaffines Spannungsniveau in Abhängigkeit vom Mittelspannungsniveau

und der Spannungsamplitude

Abbildung 5.1: Prüf- und Messaufbau für die Ermüdungsversuche der Serie II ....

Abbildung 5.2: Prüf- und Messaufbau der Kriechversuche (links) und schematischer

Versuchsaufbau (rechts)

Abbildung 5.3: Spannungsdehnungsbeziehung des untersuchten Betons

Abbildung 5.4: Bruchlastwechselzahlen der Ermüdungsversuche

Abbildung 5.5: Probekörpererwärmung im Ermüdungsversuch auf halber Probenhöhe in Abhängigkeit von der Lastwechselzahl

Abbildung 5.6: Probekörpererwärmung im Ermüdungsversuch auf halber Probenhöhe in Abhängigkeit von der Versuchsdauer

Abbildung 5.7: Entwicklung des Sekantenmoduls in Abhängigkeit von dem

Beanspruchungsniveau.

Abbildung 5.8: Bezogener Sekantenmodul in Abhängigkeit von der Bruchlastwechselzahl ........ 85

Abbildung 5.9: Sekantenmodul des ersten und letzten Entlastungsastes ..................................... 85

Abbildung 5.10: Steigung in der zweiten Phase auf dem Oberspannungsniveau ......................... 86

Abbildung 5.11: Schematische Definition der Ermüdungsbruchdehnung ................................... 87

Abbildung 5.12: Ermüdungsbruchdehnungen auf dem Oberspannungsniveau in Abhängigkeit

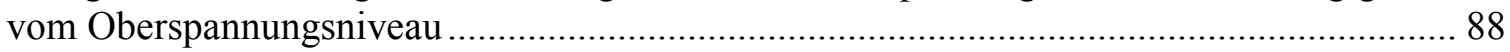

Abbildung 5.13: Ermüdungsbruchdehnungen auf dem Unterspannungsniveau in Abhängigkeit vom Oberspannungsniveau

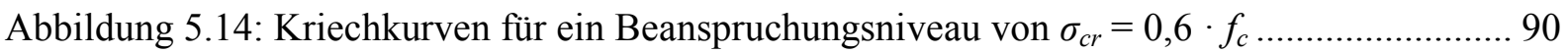

Abbildung 5.15: Verhältnis reales und rechnerisches Betonalter 
Abbildung 6.1: Ausgewählte Lastzyklen eines ermüdungsbeanspruchten Probekörpers 94

Abbildung 6.2: Elastische Dehnungsanteile zu Versuchsbeginn und Versuchsende 95

Abbildung 6.3: Prozentualer Anteil der elastischen Dehnungen an den gemessenen Ermüdungsbruchdehnungen.

Abbildung 6.4: Angenommenes Temperaturprofil zur Berechnung der mittleren Probekörpertemperatur... 96

Abbildung 6.5: Temperaturdehnungen der Ermüdungsproben bei Versuchsende. 97

Abbildung 6.6: Prozentualer Anteil der Temperaturdehnungen an den gemessenen Ermüdungsbruchdehnungen.

Abbildung 6.7: Ermüdungsbruchdehnungen korrigiert um die Temperaturdehnungen. 98

Abbildung 6.8: Viskose Dehnungen der Versuchsserie I. 100

Abbildung 6.9: Viskose Dehnungen der Versuchsserie II 100

Abbildung 6.10: Prozentualer Anteil der viskosen Dehnungen an den gemessenen Ermüdungsbruchdehnungen.

Abbildung 6.11: Ermüdungsbruchdehnungen nach der Korrektur um die thermischen und viskosen Dehnungen

Abbildung 6.12: Schädigungsinduzierter Dehnungsanteil in Abhängigkeit vom Oberspannungsniveau

Abbildung 6.13: Schädigungsinduzierte Dehnungen in Abhängigkeit der logarithmierten Bruchlastwechselzahl.

Abbildung 6.14: Schädigungsinduzierte Dehnungen in Abhängigkeit der Sekantensteifigkeit .. 104

Abbildung 6.15: Gemittelte Dehnungsanteile für die Ermüdungsversuche der Serie I mit $S_{o}=0,70, S_{u}=0,05$ und $f_{p}=1 \mathrm{~Hz}$

Abbildung 6.16: Gemittelte Dehnungsanteile für die Ermüdungsversuche der Serie I mit $S_{o}=0,70, S_{u}=0,05$ und $f_{p}=10 \mathrm{~Hz}$.

Abbildung 6.17: Prozentuale Zusammensetzung der Ermüdungsbruchdehnungen. 106

Abbildung 6.18: Verteilungsdichte der Ermüdungsbruchdehnungen 


\section{Tabellenverzeichnis}

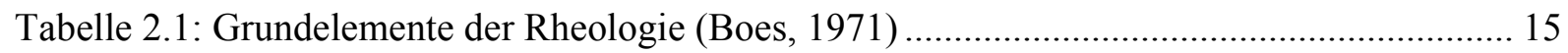

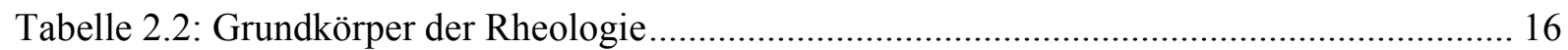

Tabelle 3.1: Zu untersuchende Spannungsniveaus im Ermüdungsversuch................................... 51

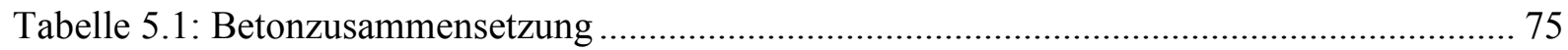

Tabelle 5.2: Beanspruchungskombinationen der durchgeführten Ermüdungsversuche ................ 76

Tabelle 5.3: Mittlere Bruchlastwechselzahlen der Ermüdungsversuche....................................... 82

Tabelle 6.1: Kriechaffine Spannungsniveaus der Ermüdungsversuche ....................................... 99 
Anhang 


\section{A.1 Ergebnisse der Druckfestigkeitsuntersuchungen}

\section{A.1.1 Würfeldruckfestigkeit}

Probenform: Betonwürfel $150 \mathrm{~mm}$ x $150 \mathrm{~mm}$ x $150 \mathrm{~mm}$

Lagerung: Wasserlagerung entsprechend DIN EN 12390-3

\begin{tabular}{|l|c|c|}
\hline Probekörper & $\begin{array}{c}\text { Prüfalter } \\
{[\mathbf{d}]}\end{array}$ & $\begin{array}{c}\text { Bruchspannung } \boldsymbol{f}_{\text {c,cube }} \\
{\left[\mathbf{N} / \mathbf{m m}^{\mathbf{2}}\right]}\end{array}$ \\
\hline 1 & 28 & $-65,86$ \\
\hline 2 & 28 & $-67,73$ \\
\hline 3 & 28 & $-63,07$ \\
\hline Minimalwert & $-67,73$ \\
\hline Maximalwert & $-63,07$ \\
\hline Mittelwert & $\mathbf{- 6 5 , 5 5}$ \\
\hline
\end{tabular}

\section{A.1.2 Zylinderdruckfestigkeit}

Probenform: Betonzylinder $\varnothing=103 \mathrm{~mm}, \mathrm{H}=280 \mathrm{~mm}$

Lagerung: $\quad$ Entformen nach 48 Stunden, anschließend Lagerung für mindestens 14 Tage bei $20{ }^{\circ} \mathrm{C}$ und $65 \%$ rel. Luftfeuchtigkeit

\begin{tabular}{|l|c|c|c|}
\hline Probekörper & $\begin{array}{c}\text { Prüfalter } \\
{[\mathbf{d}]}\end{array}$ & $\begin{array}{c}\text { Bruchspannung } \boldsymbol{f}_{\boldsymbol{c}} \\
{\left[\mathbf{N} / \mathbf{m m}^{\mathbf{2}}\right]}\end{array}$ & $\begin{array}{c}\text { Bruchdehnung } \boldsymbol{\varepsilon}_{\boldsymbol{c}} \\
{[\mathbf{\% o}]}\end{array}$ \\
\hline PK 12 & 44 & $-70,00$ & $-3,649$ \\
\hline PK 17 & 44 & $-71,93$ & $-3,717$ \\
\hline PK 003 & 44 & $-68,77$ & $-3,599$ \\
\hline PK 019 & 132 & $-70,36$ & $-3,405$ \\
\hline PK 020 & 132 & $-68,23$ & $-3,303$ \\
\hline PK 028 & 132 & $-74,36$ & $-3,498$ \\
\hline Minimalwert & & $-74,36$ & $-3,717$ \\
\hline Maximalwert & & $-68,23$ & $-3,303$ \\
\hline Mittelwert & & $\mathbf{- 7 0 , 6 1}$ & $\mathbf{- 3 , 5 2 8}$ \\
\hline
\end{tabular}




\section{A.2 Ermüdungsversuche}

\section{A.2.1 Probekörperübersicht und Bruchlastwechselzahlen}

\begin{tabular}{|c|c|c|c|c|c|c|c|}
\hline $\begin{array}{l}\text { Probe- } \\
\text { körper }\end{array}$ & Serie & $S_{o}$ & $S_{u}$ & $f_{p}$ & $N_{f}$ & $\log N_{f}$ & Bemerkung \\
\hline & & - & - & $\mathrm{Hz}$ & - & - & \\
\hline PK 001 & 1 & 0,80 & 0,05 & 10 & 4.803 & 3,682 & \\
\hline PK 002 & 1 & 0,80 & 0,05 & 10 & 3.912 & 3,592 & $\mathrm{~T}$ \\
\hline PK 11 & 1 & 0,80 & 0,05 & 10 & 2.137 & 3,330 & $\mathrm{~T}$ \\
\hline PK 10 & 1 & 0,80 & 0,05 & 10 & 793 & 2,899 & \\
\hline PK 20 & 1 & 0,80 & 0,05 & 10 & 5.450 & 3,736 & \\
\hline PK 004 & 1 & 0,70 & 0,05 & 10 & 28.807 & 4,459 & \\
\hline PK 14 & 1 & 0,70 & 0,05 & 10 & 41.748 & 4,621 & $\mathrm{~T}$ \\
\hline PK 007 & 1 & 0,70 & 0,05 & 10 & 27.215 & 4,435 & $\mathrm{~T}$ \\
\hline PK 19 & 1 & 0,70 & 0,05 & 10 & 32.622 & 4,514 & \\
\hline PK 24 & 1 & 0,60 & 0,05 & 10 & 94.223 & 4,974 & $\mathrm{~T}$ \\
\hline PK 21 & 1 & 0,60 & 0,05 & 10 & 306.326 & 5,486 & \\
\hline PK 23 & 1 & 0,60 & 0,05 & 10 & 105.574 & 5,024 & $\mathrm{~T}$ \\
\hline PK 016 & 1 & 0,60 & 0,05 & 10 & 146.527 & 5,166 & \\
\hline PK 012 & 1 & 0,80 & 0,05 & 1 & 2.507 & 3,399 & $\mathrm{~T}$ \\
\hline PK 009 & 1 & 0,80 & 0,05 & 1 & 2.482 & 3,395 & $\mathrm{~T}$ \\
\hline PK 014 & 1 & 0,80 & 0,05 & 1 & 1.192 & 3,076 & \\
\hline PK 16 & 1 & 0,70 & 0,05 & 1 & 92.228 & 4,965 & $\mathrm{~T}$ \\
\hline PK 018 & 1 & 0,70 & 0,05 & 1 & 118.044 & 5,072 & $\mathrm{~T}$ \\
\hline PK 015 & 1 & 0,60 & 0,05 & 1 & 290.500 & 5,463 & $\mathrm{~T}, \mathrm{D}$ \\
\hline PK 013 & 1 & 0,60 & 0,05 & 1 & 306.200 & 5,486 & $\mathrm{D}$ \\
\hline PK 26 & 2 & 0,70 & 0,05 & 10 & 47.677 & 4,678 & \\
\hline PK 039 & 2 & 0,70 & 0,05 & 10 & 44.010 & 4,644 & \\
\hline PK 046 & 2 & 0,70 & 0,05 & 10 & 41.913 & 4,622 & \\
\hline PK 22 & 2 & 0,70 & 0,05 & 10 & 38.238 & 4,582 & $\mathrm{~T}$ \\
\hline PK 040 & 2 & 0,70 & 0,05 & 10 & 42.492 & 4,628 & $\mathrm{~T}$ \\
\hline PK 13 & 2 & 0,60 & 0,05 & 10 & 262.878 & 5,420 & \\
\hline PK 047 & 2 & 0,60 & 0,05 & 10 & 636.601 & 5,804 & \\
\hline PK 011 & 2 & 0,60 & 0,05 & 10 & 206.069 & 5,314 & $\mathrm{~T}$ \\
\hline PK 043 & 2 & 0,60 & 0,05 & 10 & 288.333 & 5,460 & $\mathrm{~T}$ \\
\hline PK 041 & 2 & 0,60 & 0,05 & 10 & 2.085 .780 & 6,319 & $\mathrm{~A}$ \\
\hline
\end{tabular}
A: Ausreißer
D: Durchläufer
T: Temperaturmessung durchgeführt 


\section{A.2.2 Probekörpererwärmung}

Die folgende Tabelle listet die Temperaturänderungen der ermüdungsbeanspruchten Probeköper im oberen und unteren Bereich der Probekörper sowie auf halber Probenhöhen auf. Dargestellt sind die Oberflächentemperaturänderungen zwischen Versuchsbeginn und Versuchsende an den genannten Messstellen. Des Weiteren sind die mittleren Temperaturänderungen $\Delta T_{\text {dehn }}$ (Gleichung 6.1) und die resultierenden Temperaturdehnungen $\varepsilon_{t}^{B}$ (Gleichung 6.2) aufgeführt. Auf den folgenden Seiten werden die Temperaturverläufe der Probekörper abgebildet. Für einige Beanspruchungsgruppen konnten Ausgleichskurven bestimmt werden, die in den Grafiken enthalten sind. Auf Grundlage dieser Ausgleichskurven wurden für die Probekörper, bei denen keine Temperaturmessungen erfolgten, die Oberflächentemperaturen an den genannten Messstellen berechnet und in der Tabelle gräulich dargestellt. Bei der Beanspruchungsgruppe $S_{o}=0,60 / S_{u}=0,05 / f_{p}=10 \mathrm{~Hz}$ entsprechen die rechnerisch angesetzten Temperaturen den mittleren Temperaturänderungen der Beanspruchungsgruppe.

\begin{tabular}{|c|c|c|c|c|c|c|c|}
\hline$S_{o} / S_{u} / f_{p}$ & $\begin{array}{l}\text { Probe- } \\
\text { körper }\end{array}$ & $\begin{array}{l}\text { Versuchs } \\
\text {-dauer }\end{array}$ & $\Delta \boldsymbol{T}_{\text {oben }}$ & $\Delta \boldsymbol{T}_{\text {mitte }}$ & $\Delta \boldsymbol{T}_{\text {unten }}$ & $\Delta \boldsymbol{T}_{\boldsymbol{d e h n}}$ & $\varepsilon_{t}^{B}$ \\
\hline & & $\mathrm{s}$ & $\mathrm{K}$ & $\mathrm{K}$ & $\mathrm{K}$ & $\mathrm{K}$ & $\%$ \\
\hline \multirow{5}{*}{$0,80 / 0,05 / 10$} & PK 001 & 480 & 4,95 & 6,96 & 4,95 & 7,15 & 0,072 \\
\hline & PK 002 & 391 & 3,19 & 5,66 & 3,63 & 5,59 & 0,056 \\
\hline & PK 11 & 214 & 2,11 & 3,35 & 1,79 & 3,29 & 0,033 \\
\hline & PK 10 & 79 & 0,82 & 1,15 & 0,82 & 1,18 & 0,012 \\
\hline & PK 20 & 545 & 5,61 & 7,90 & 5,61 & 8,12 & 0,081 \\
\hline \multirow{9}{*}{$0,70 / 0,05 / 10$} & PK 004 & 2.881 & 7,14 & 22,18 & 7,14 & 19,62 & 0,196 \\
\hline & PK 14 & 4.175 & 11,58 & 34,30 & 12,50 & 30,71 & 0,307 \\
\hline & PK 007 & 2.722 & 7,94 & 24,88 & 6,00 & 21,63 & 0,216 \\
\hline & PK 19 & 3.262 & 7,70 & 25,12 & 7,70 & 22,08 & 0,221 \\
\hline & PK 26 & 4.768 & 9,66 & 36,71 & 9,66 & 31,69 & 0,317 \\
\hline & PK 039 & 4.401 & 9,21 & 33,89 & 9,21 & 29,35 & 0,294 \\
\hline & PK 046 & 4.191 & 8,94 & 32,27 & 8,94 & 28,02 & 0,280 \\
\hline & PK 22 & 3.824 & 5,98 & 28,42 & 6,20 & 24,02 & 0,240 \\
\hline & PK 040 & 4.249 & 6,67 & 31,13 & 7,40 & 26,44 & 0,264 \\
\hline \multirow{8}{*}{$0,60 / 0,05 / 10$} & PK 24 & 9.422 & 10,81 & 37,34 & 7,90 & 32,05 & 0,321 \\
\hline & PK 21 & 30.633 & 10,80 & 43,20 & 10,80 & 37,07 & 0,371 \\
\hline & PK 23 & 10.557 & 11,74 & 46,25 & 11,95 & 39,79 & 0,398 \\
\hline & PK 016 & 14.653 & 10,80 & 43,20 & 10,80 & 37,07 & 0,371 \\
\hline & PK 13 & 26.288 & 10,80 & 43,20 & 10,80 & 37,07 & 0,371 \\
\hline & PK 047 & 63.660 & 10,80 & 43,20 & 10,80 & 37,07 & 0,371 \\
\hline & PK 011 & 20.607 & 6,35 & 47,53 & 12,67 & 39,93 & 0,399 \\
\hline & PK 043 & 28.833 & 10,32 & 41,68 & 14,65 & 36,53 & 0,365 \\
\hline \multirow{3}{*}{$0,80 / 0,05 / 1$} & PK 012 & 2.507 & 1,08 & 3,63 & 1,32 & 3,22 & 0,032 \\
\hline & PK 009 & 2.482 & 1,92 & 3,55 & 1,85 & 3,41 & 0,034 \\
\hline & PK 014 & 1.192 & 0,93 & 1,61 & 0,93 & 1,57 & 0,016 \\
\hline \multirow{2}{*}{$0,70 / 0,05 / 1$} & PK 16 & 92.228 & 3,57 & 9,99 & 4,12 & 9,07 & 0,091 \\
\hline & PK 018 & 118.044 & 2,13 & 9,64 & 3,67 & 8,45 & 0,085 \\
\hline $0,60 / 0,05 / 1$ & PK 015 & 290.500 & 1,23 & 5,66 & 2,46 & 5,02 & 0,050 \\
\hline
\end{tabular}


$S_{o}=0,80 / S_{u}=0,05 / f_{p}=10 \mathrm{~Hz}$

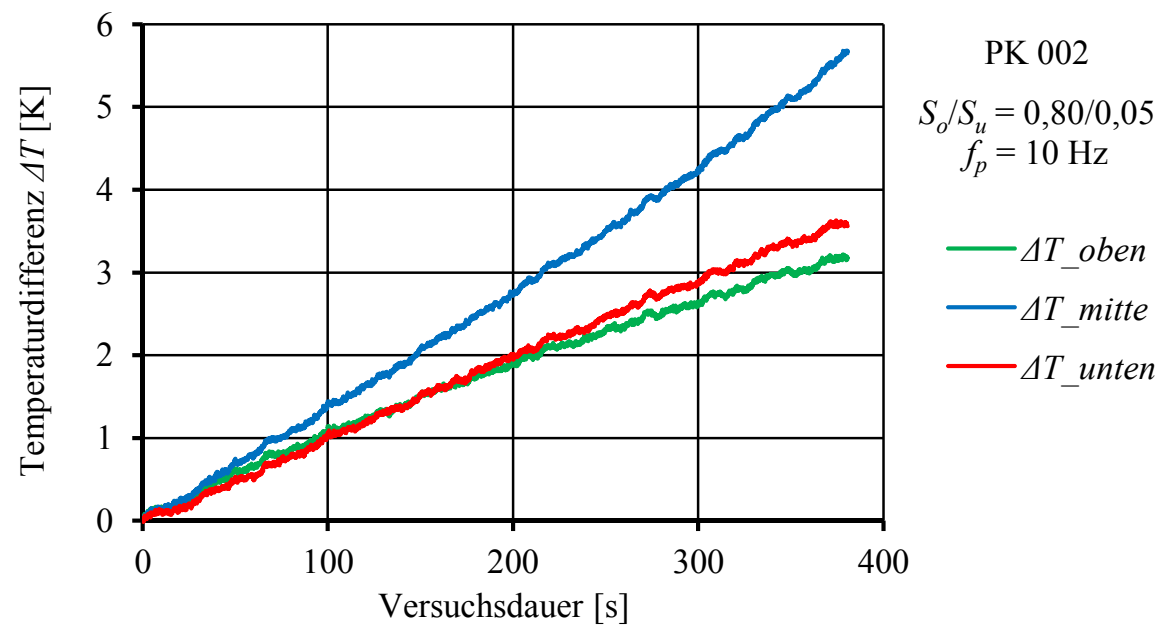

Abbildung A. 2.1: Probekörpererwärmung PK 002, $S_{o}=0,80 / S_{u}=0,05 / f_{p}=10 \mathrm{~Hz}$

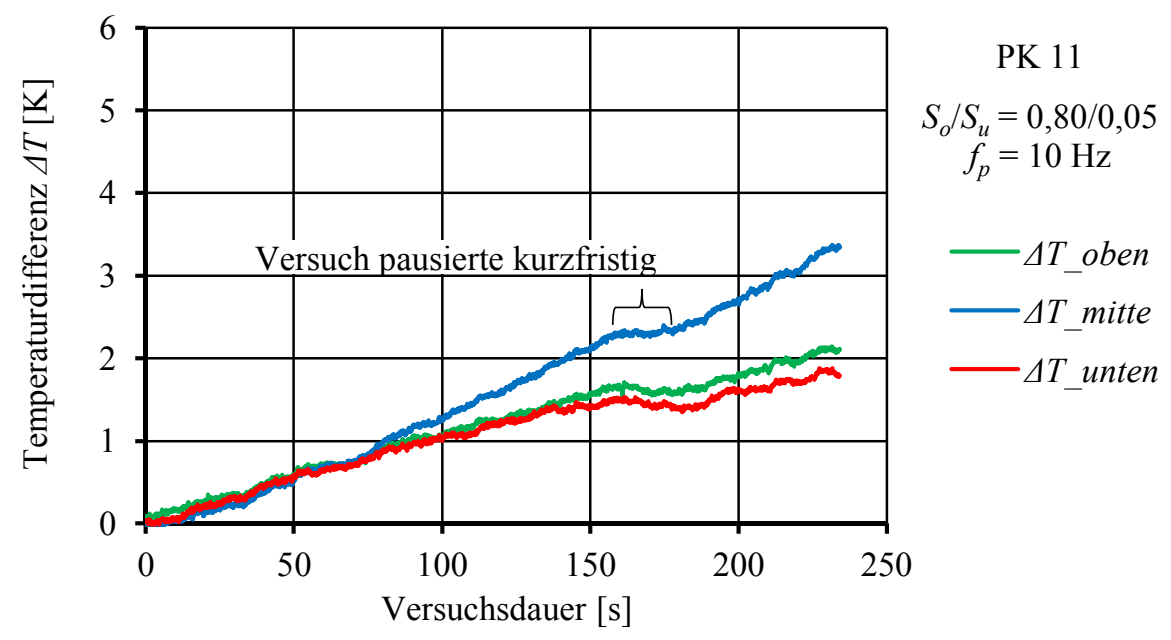

Abbildung A. 2.2: Probekörpererwärmung PK 11, $S_{o}=0,80 / S_{u}=0,05 / f_{p}=10 \mathrm{~Hz}$

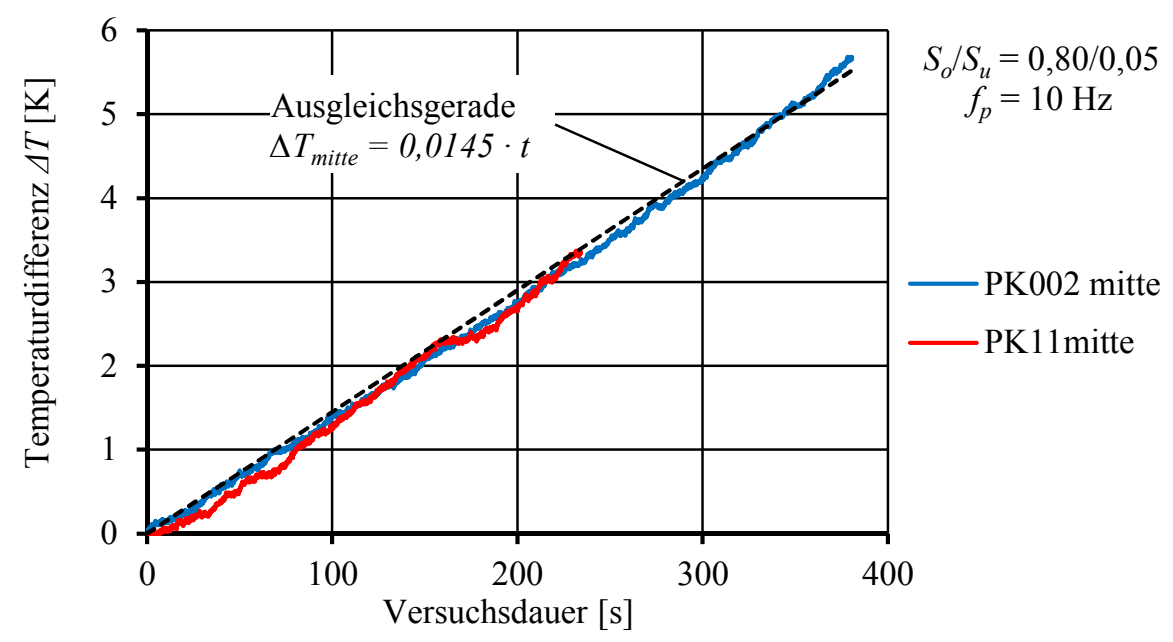

Abbildung A. 2.3: Probekörpererwärmung auf halber Probenhöhe, $S_{o}=0,80 / S_{u}=0,05 / f_{p}=10 \mathrm{~Hz}$ 


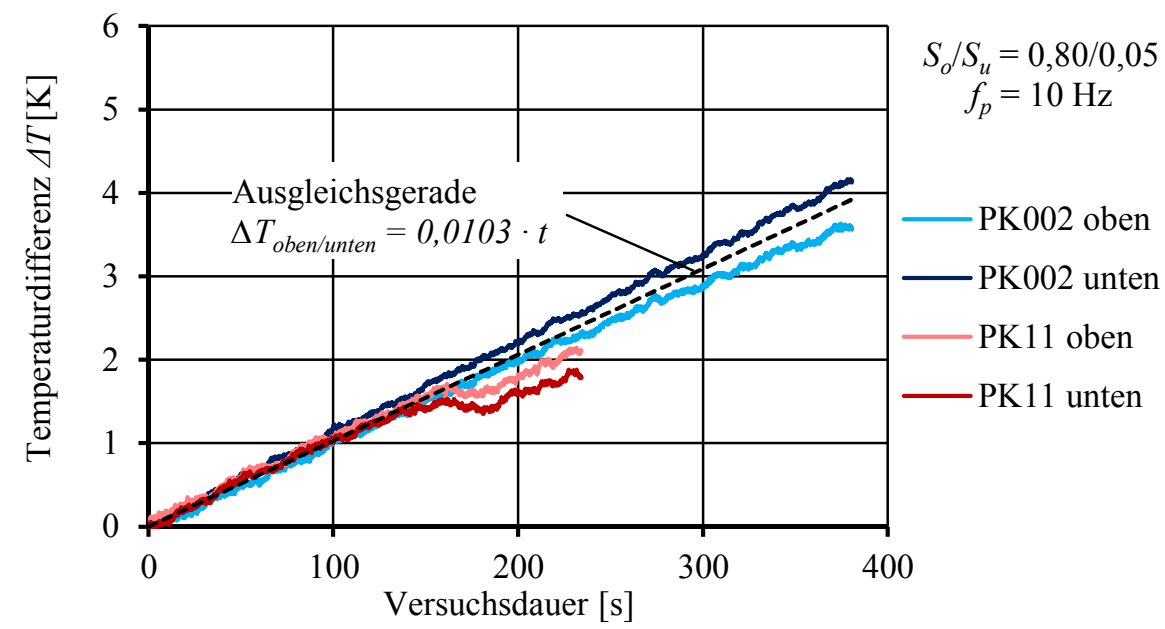

Abbildung A. 2.4: Probekörpererwärmung am oberen und unteren Probenrand, $S_{o}=0,80 / S_{u}=0,05 / f_{p}=10 \mathrm{~Hz}$ 
$S_{o}=0,70 / S_{u}=0,05 / f_{p}=10 \mathrm{~Hz}$

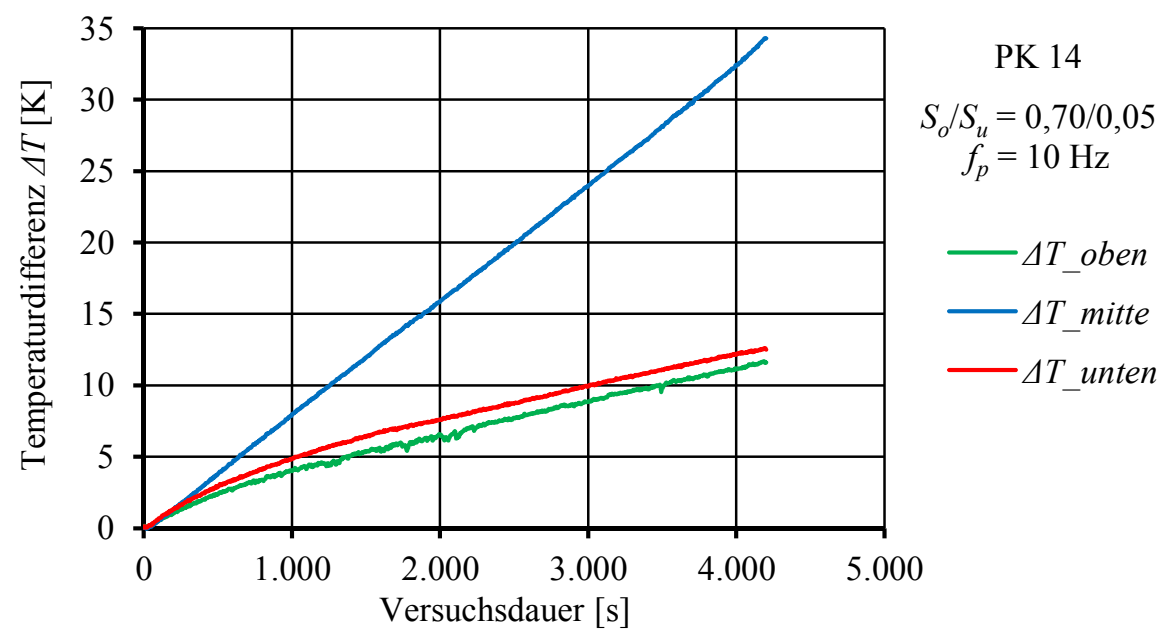

Abbildung A. 2.5: Probekörpererwärmung PK 14, $S_{o}=0,70 / S_{u}=0,05 / f_{p}=10 \mathrm{~Hz}$

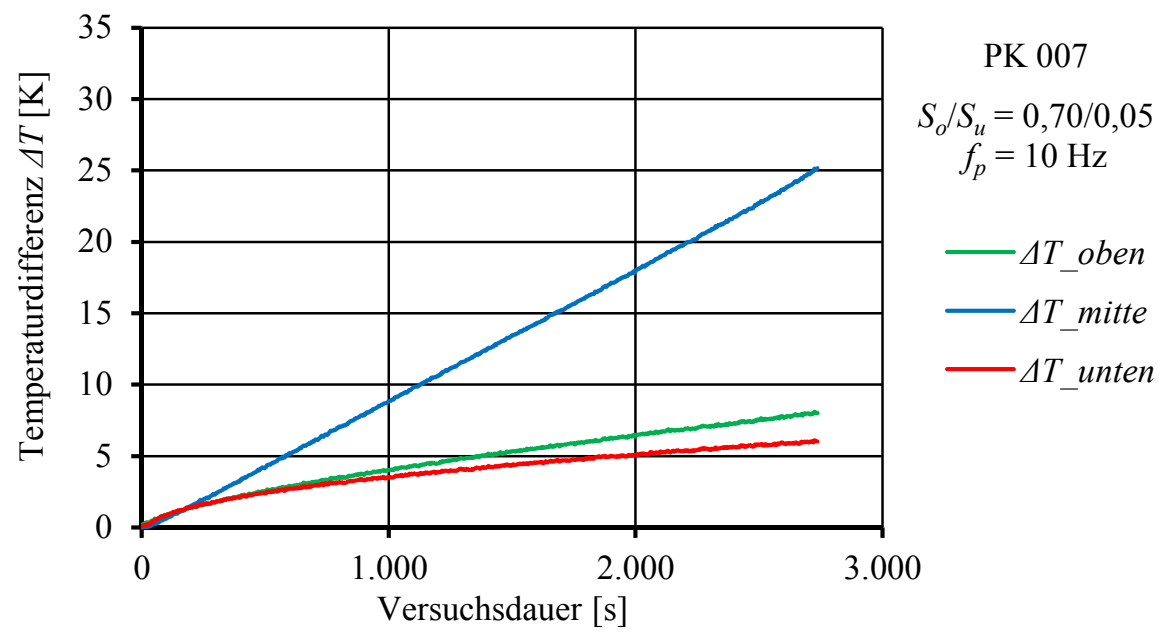

Abbildung A. 2.6: Probekörpererwärmung PK 007, $S_{o}=0,70 / S_{u}=0,05 / f_{p}=10 \mathrm{~Hz}$

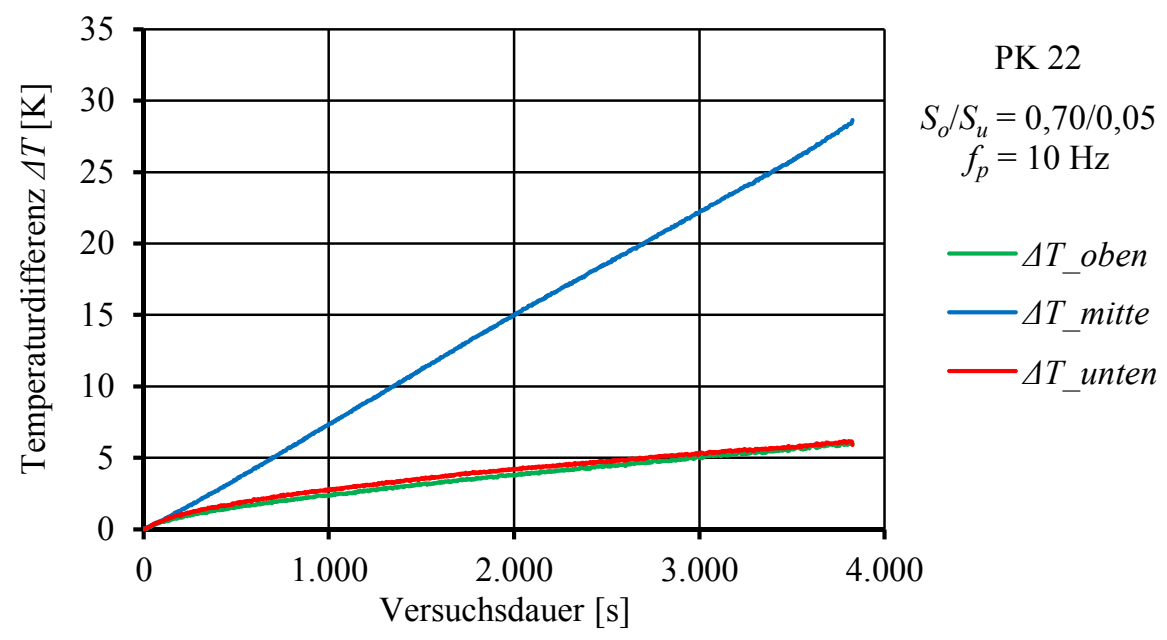

Abbildung A. 2.7: Probekörpererwärmung PK 22, $S_{o}=0,70 / S_{u}=0,05 / f_{p}=10 \mathrm{~Hz}$ 


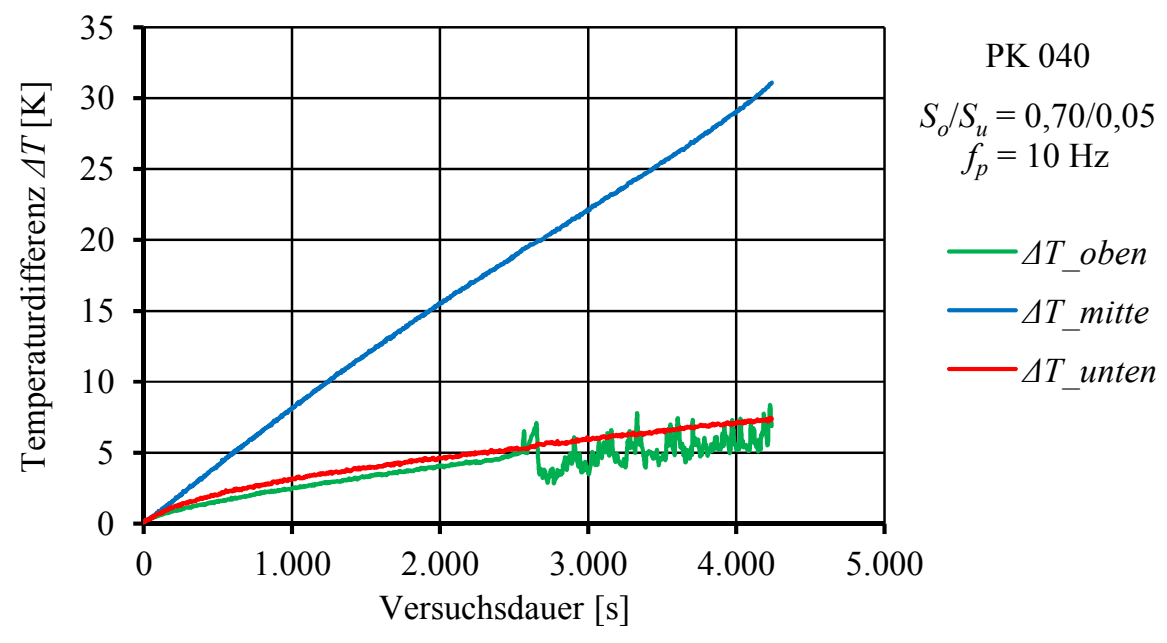

Abbildung A. 2.8: Probekörpererwärmung PK 040, $S_{o}=0,70 / S_{u}=0,05 / f_{p}=10 \mathrm{~Hz}$

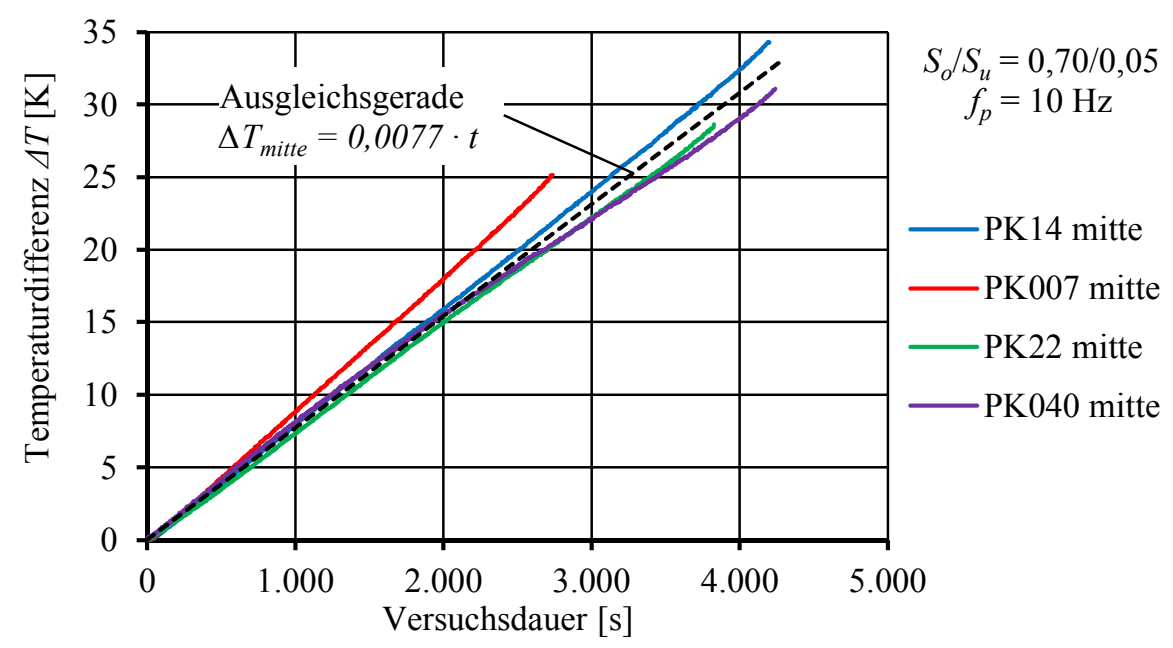

Abbildung A. 2.9: Probekörpererwärmung auf halber Probenhöhe, $S_{o}=0,70 / S_{u}=0,05 / f_{p}=10 \mathrm{~Hz}$

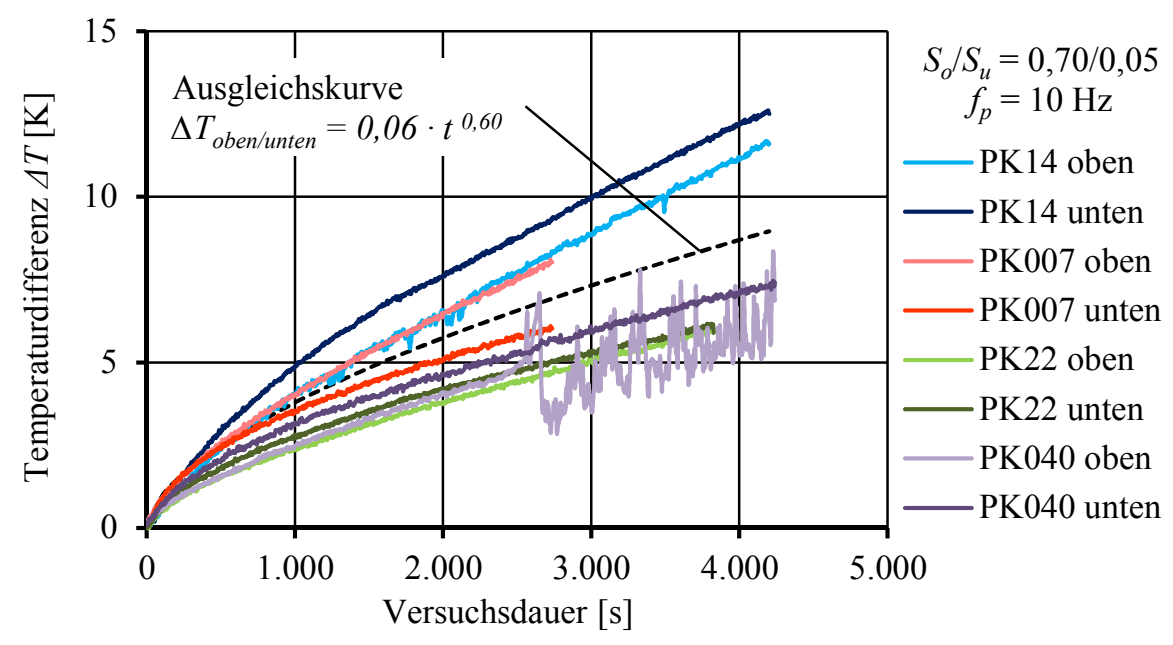

Abbildung A. 2.10: Probekörpererwärmung am oberen und unteren Probenrand, $S_{o}=0,70 / S_{u}=0,05 / f_{p}=10 \mathrm{~Hz}$ 
$S_{o}=0,60 / S_{u}=0,05 / f_{p}=10 \mathrm{~Hz}$

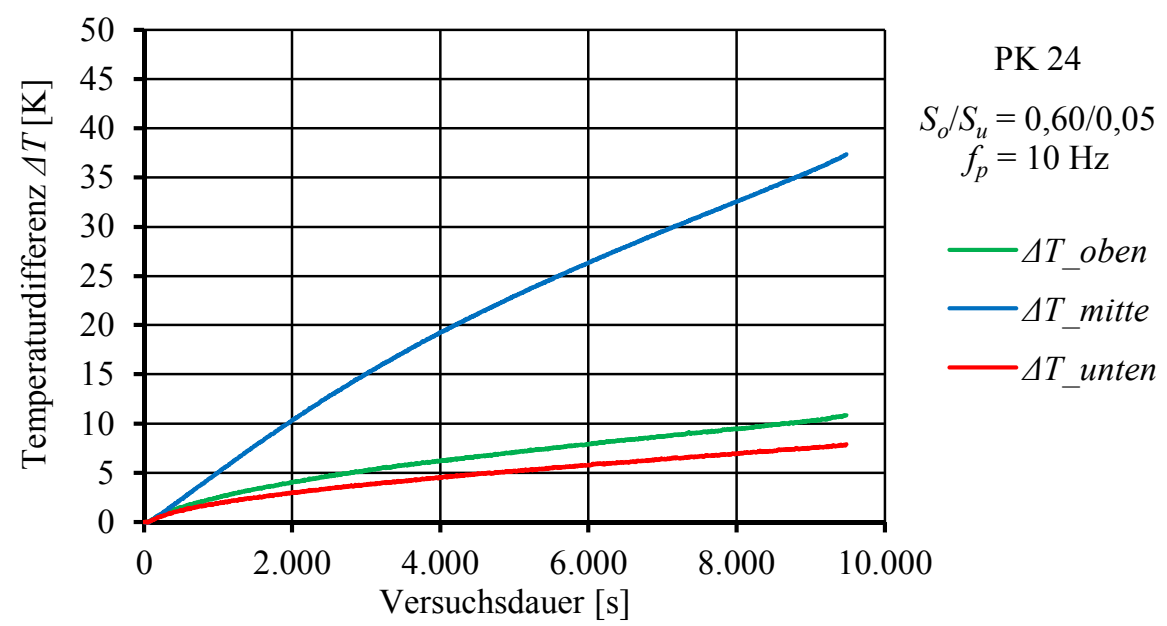

Abbildung A. 2.11: Probekörpererwärmung PK 24, $S_{o}=0,60 / S_{u}=0,05 / f_{p}=10 \mathrm{~Hz}$

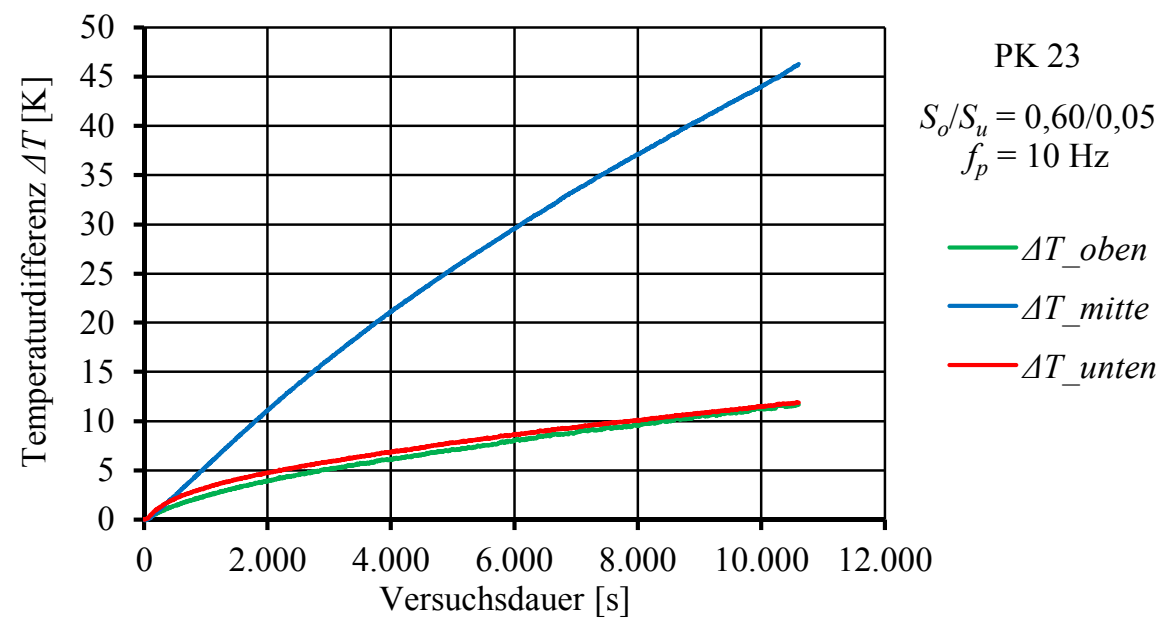

Abbildung A. 2.12: Probekörpererwärmung PK 23, $S_{o}=0,60 / S_{u}=0,05 / f_{p}=10 \mathrm{~Hz}$

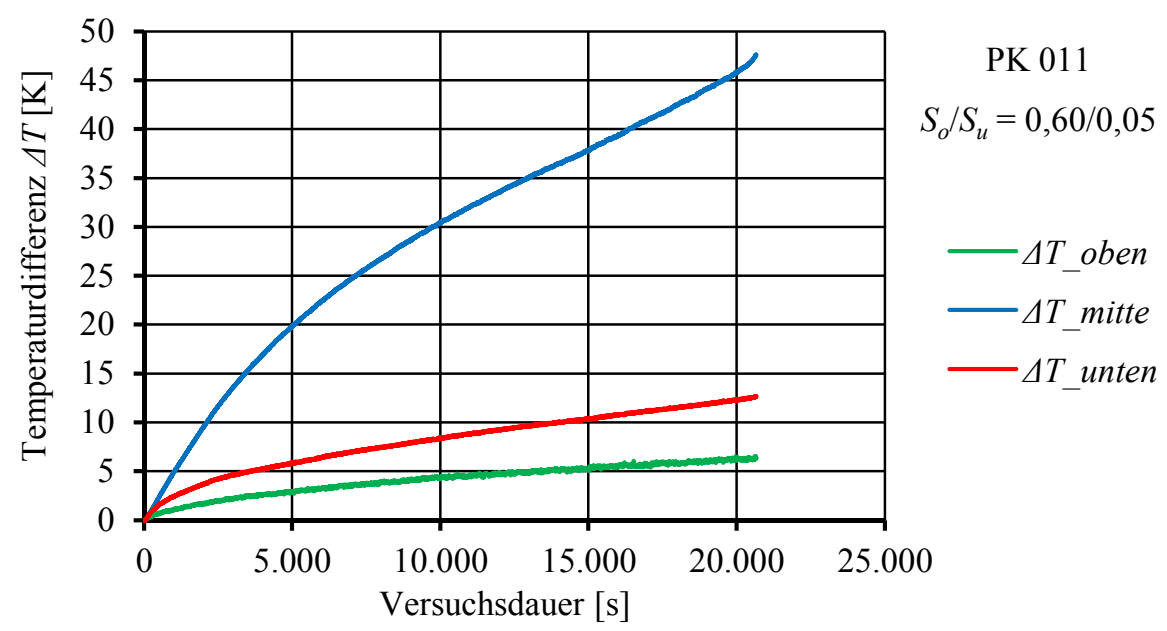

Abbildung A. 2.13: Probekörpererwärmung PK 011, $S_{o}=0,60 / S_{u}=0,05 / f_{p}=10 \mathrm{~Hz}$ 


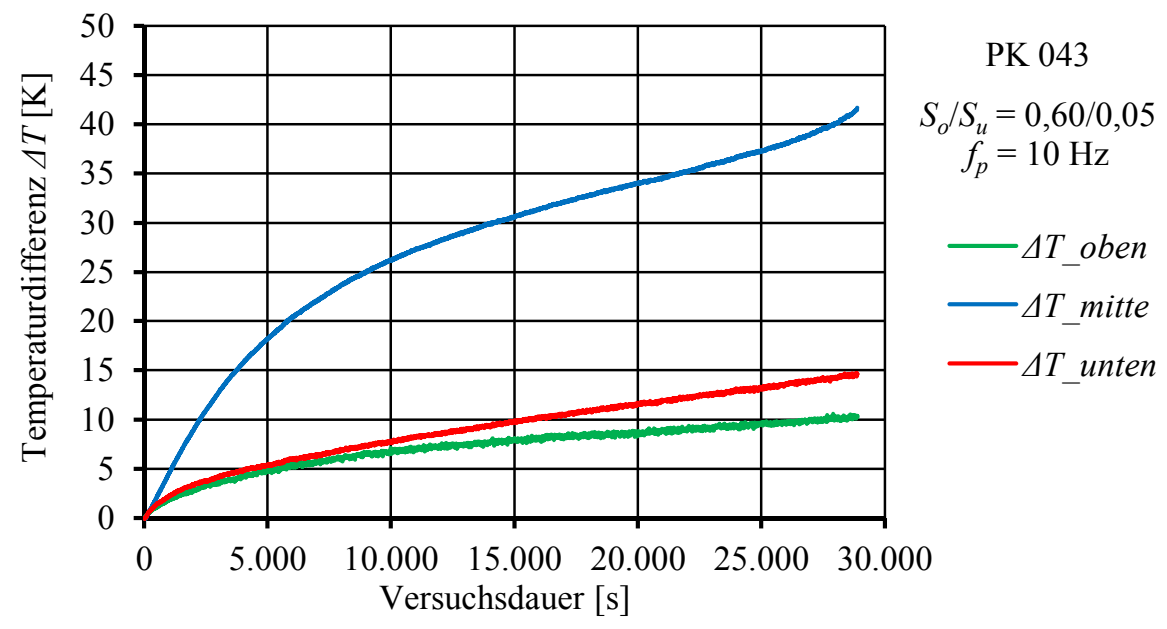

Abbildung A. 2.14: Probekörpererwärmung PK 043, $S_{o}=0,60 / S_{u}=0,05 / f_{p}=10 \mathrm{~Hz}$

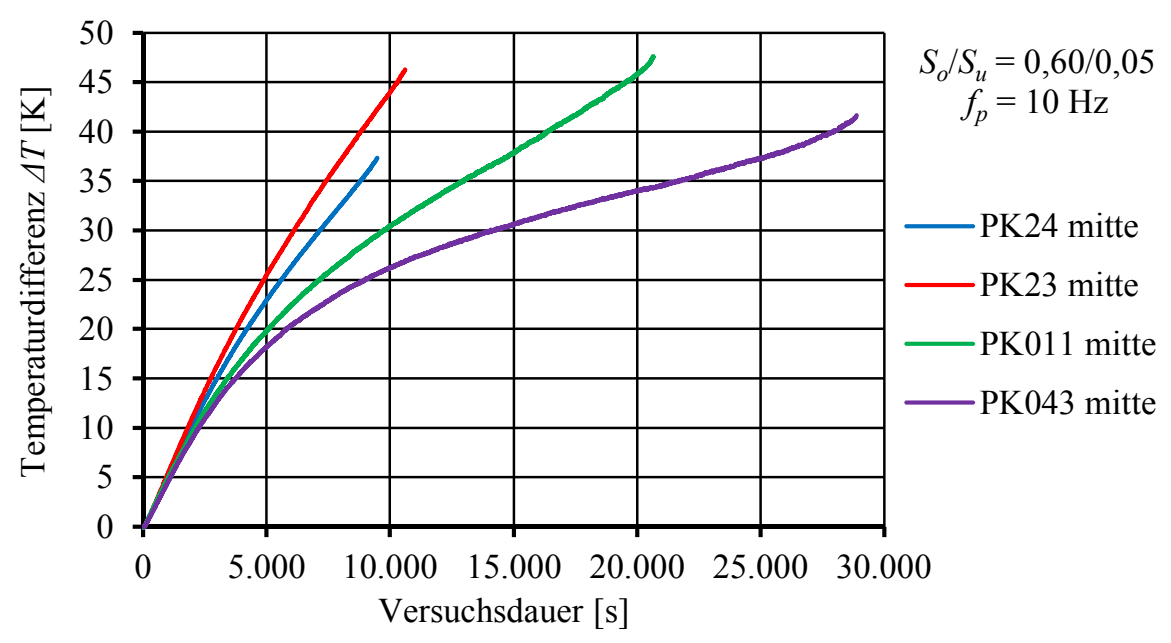

Abbildung A. 2.15: Probekörpererwärmung auf halber Probenhöhe, $S_{o}=0,60 / S_{u}=0,05 / f_{p}=10 \mathrm{~Hz}$

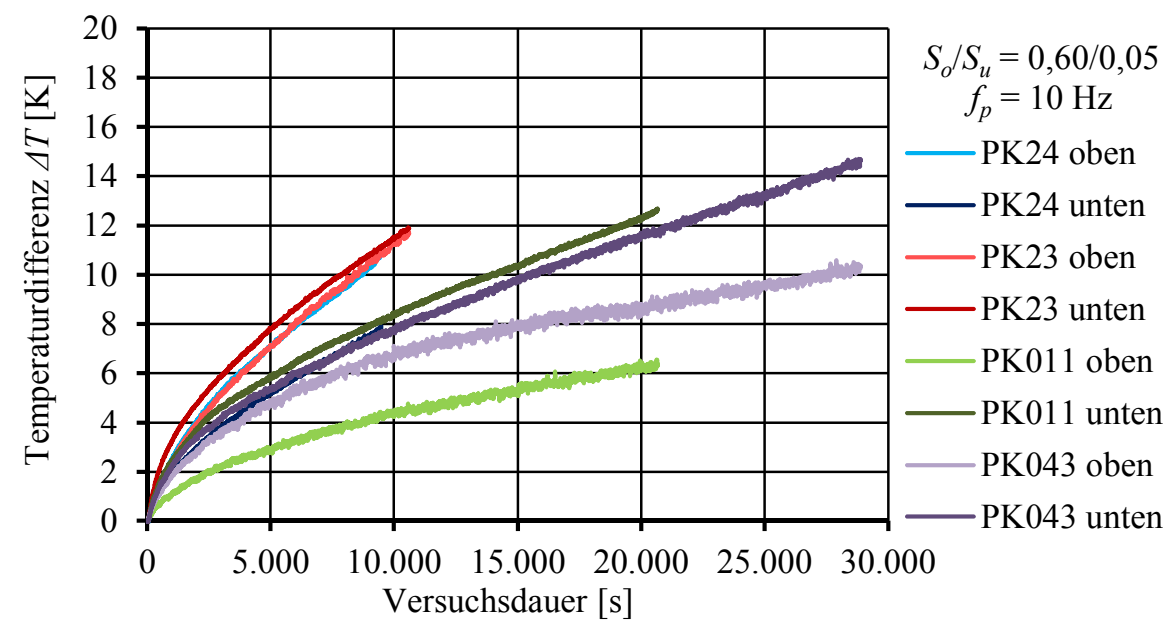

Abbildung A. 2.16: Probekörpererwärmung am oberen und unteren Probenrand, $S_{o}=0,60 / S_{u}=0,05 / f_{p}=10 \mathrm{~Hz}$ 
$S_{o}=0,80 / S_{u}=0,05 / f_{p}=1 \mathrm{~Hz}$

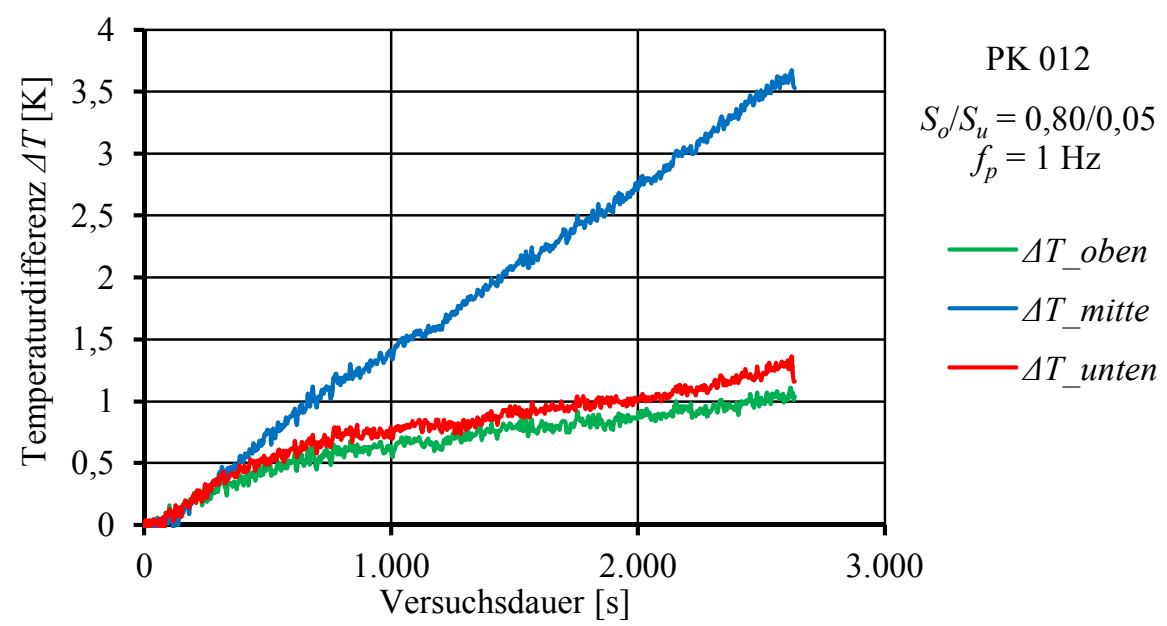

Abbildung A. 2.17: Probekörpererwärmung PK 012, $S_{o}=0,80 / S_{u}=0,05 / f_{p}=1 \mathrm{~Hz}$

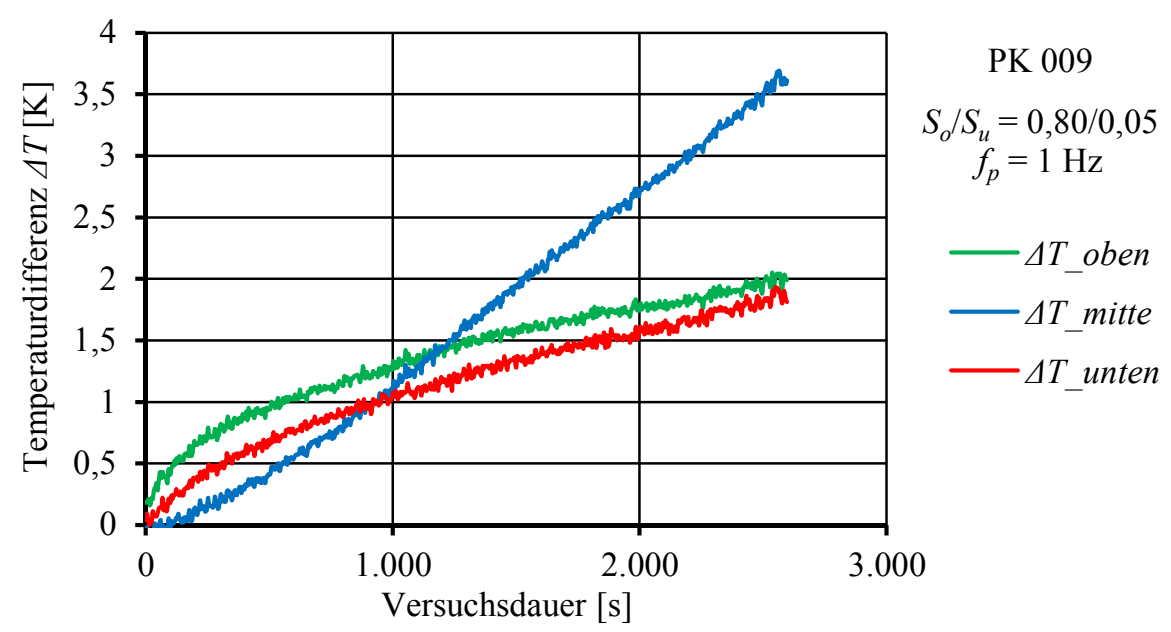

Abbildung A. 2.18: Probekörpererwärmung PK 009, $S_{o}=0,80 / S_{u}=0,05 / f_{p}=1 \mathrm{~Hz}$

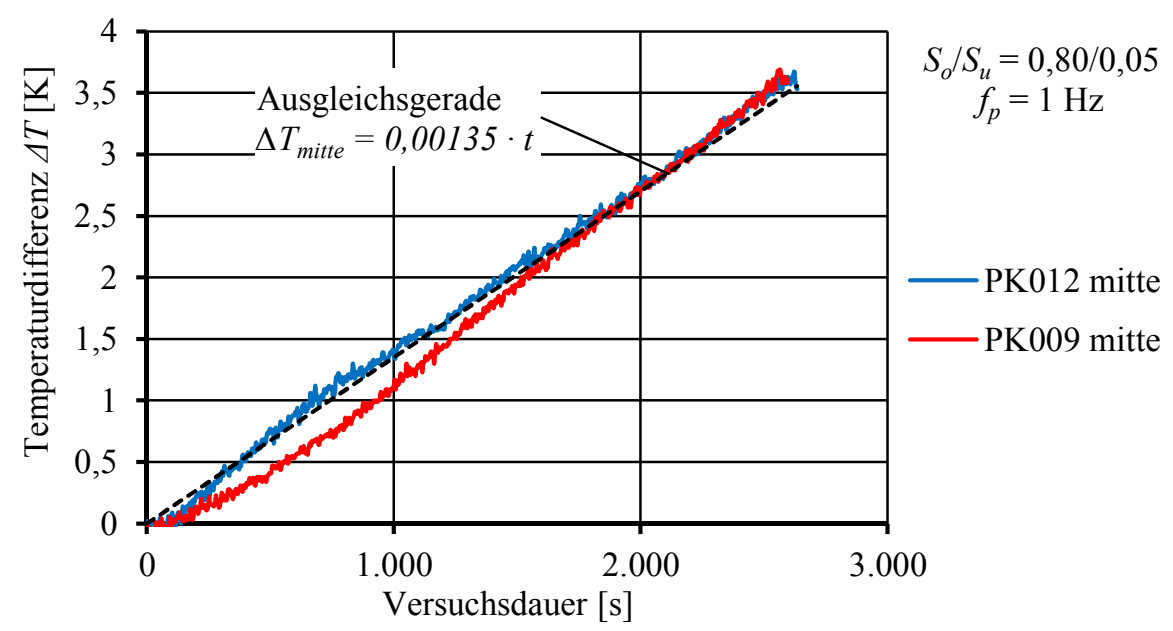

Abbildung A. 2.19: Probekörpererwärmung auf halber Probenhöhe, $S_{o}=0,80 / S_{u}=0,05 / f_{p}=1 \mathrm{~Hz}$ 


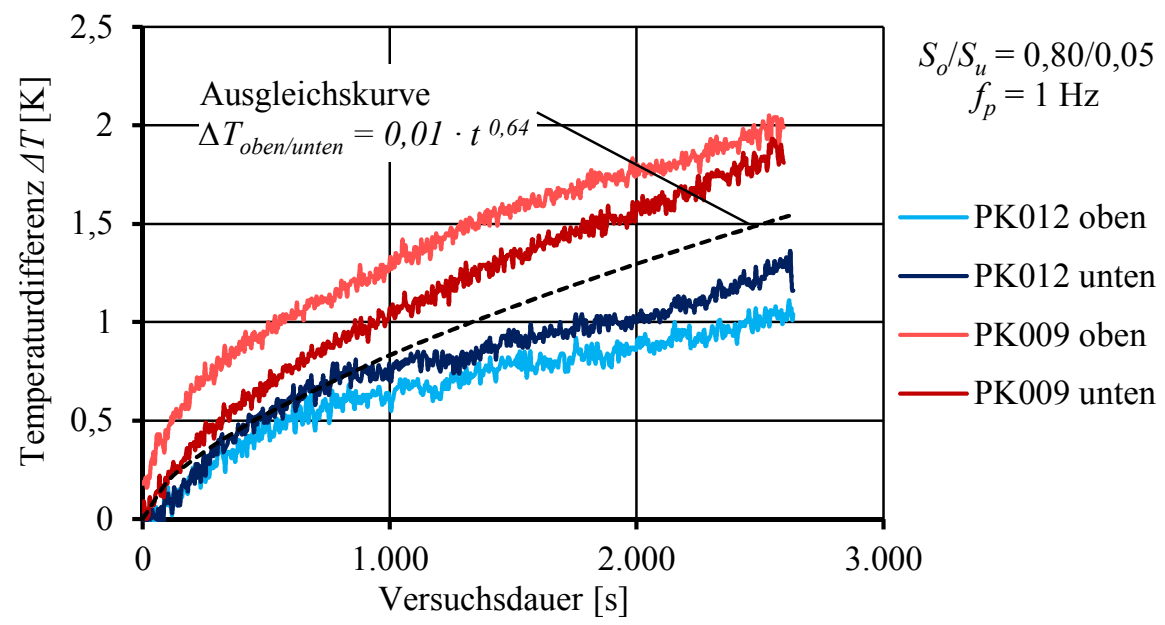

Abbildung A. 2.20: Probekörpererwärmung am oberen und unteren Probenrand, $S_{o}=0,80 / S_{u}=0,05 / f_{p}=1 \mathrm{~Hz}$ 
$S_{o}=0,70 / S_{u}=0,05 / f_{p}=1 \mathrm{~Hz}$

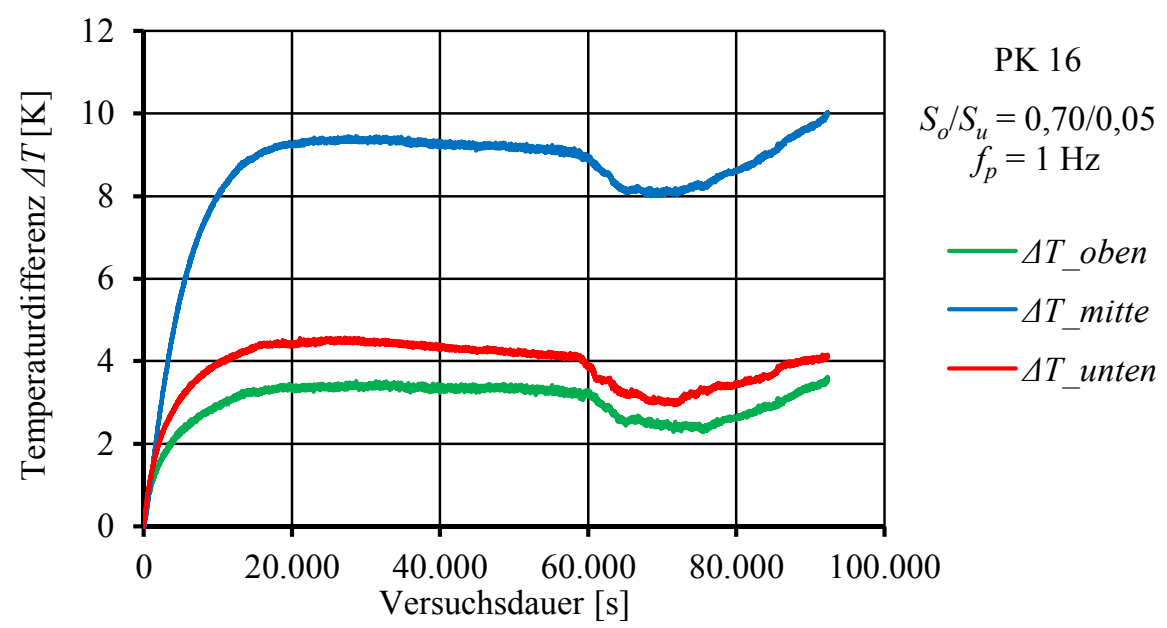

Abbildung A. 2.21: Probekörpererwärmung PK 16, $S_{o}=0,70 / S_{u}=0,05 / f_{p}=1 \mathrm{~Hz}$

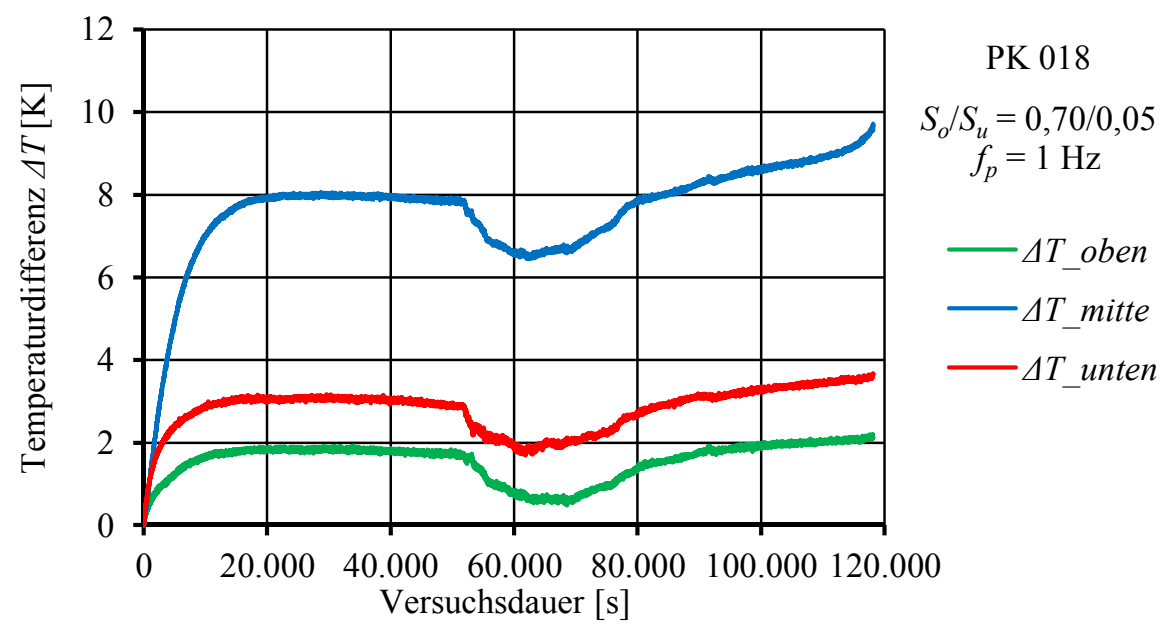

Abbildung A. 2.22: Probekörpererwärmung PK 018, $S_{o}=0,70 / S_{u}=0,05 / f_{p}=1 \mathrm{~Hz}$

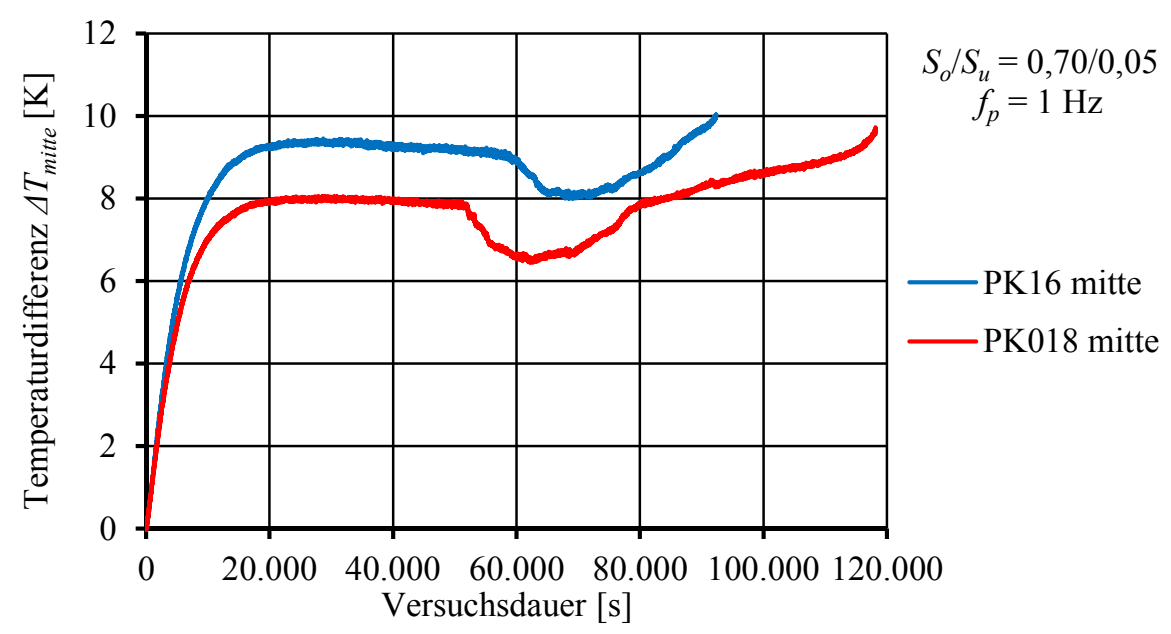

Abbildung A. 2.23: Probekörpererwärmung auf halber Probenhöhe, $S_{o}=0,70 / S_{u}=0,05 / f_{p}=1 \mathrm{~Hz}$ 


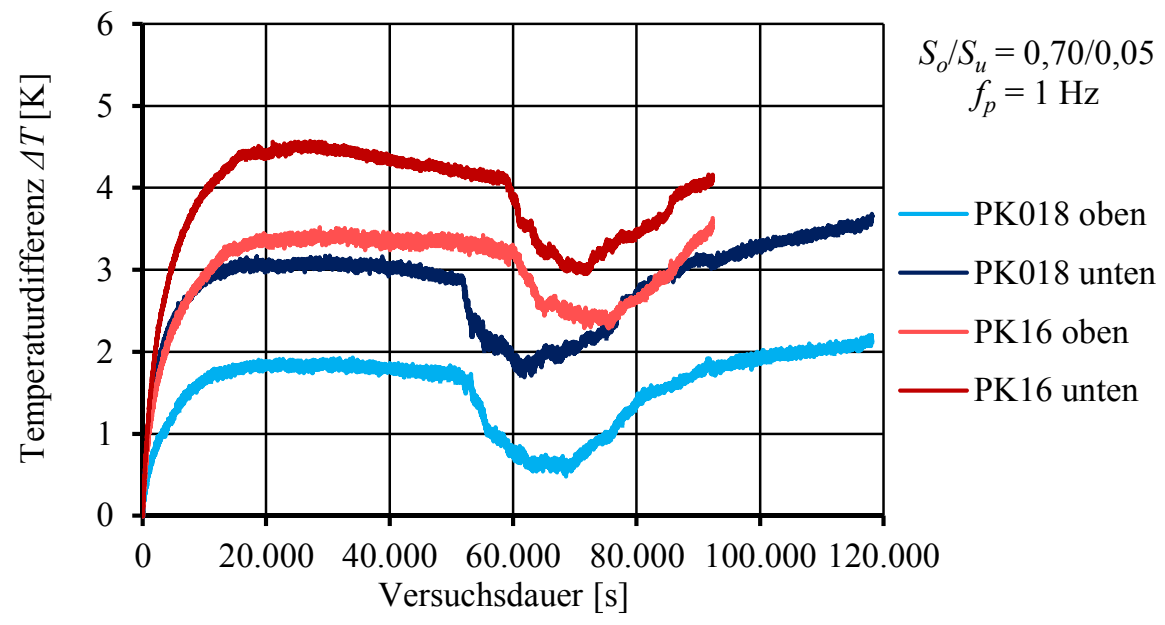

Abbildung A. 2.24: Probekörpererwärmung auf halber Probenhöhe, $S_{o}=0,70 / S_{u}=0,05 / f_{p}=1 \mathrm{~Hz}$ 
$S_{o}=0,60 / S_{u}=0,05 / f_{p}=1 \mathrm{~Hz}$

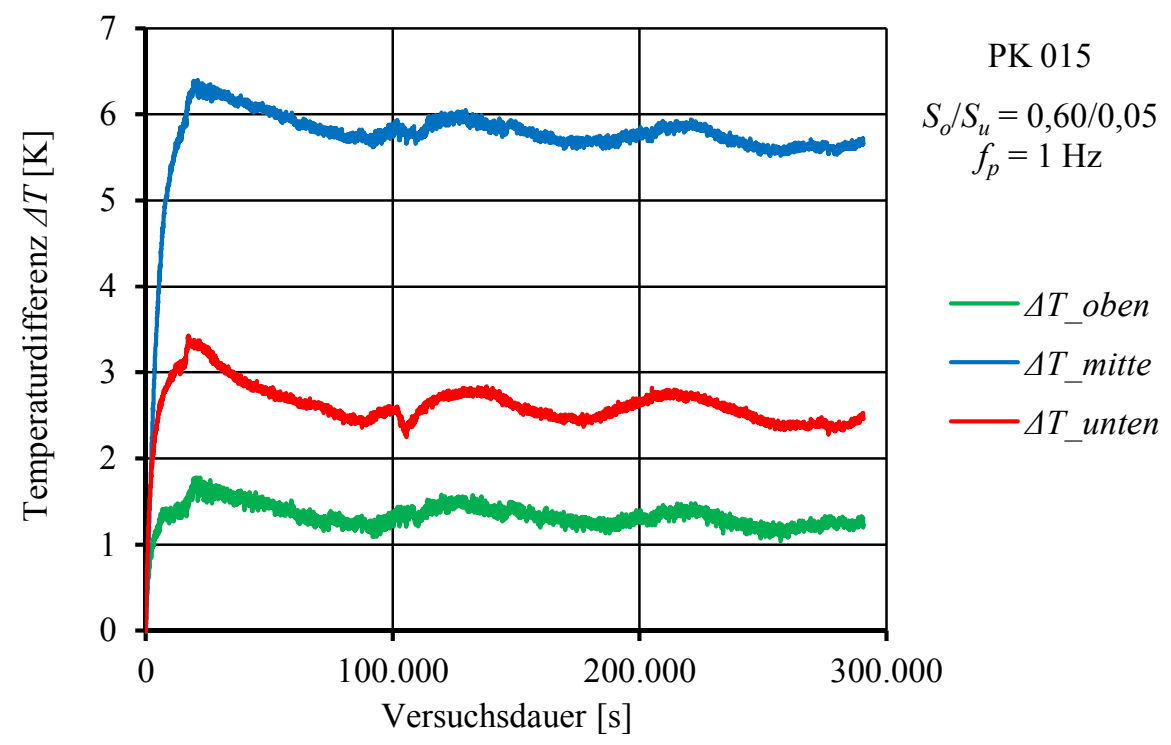

Abbildung A. 2.25: Probekörpererwärmung PK 015, $S_{o}=0,60 / S_{u}=0,05 / f_{p}=1 \mathrm{~Hz}$ 


\section{A.2.3 Steifigkeitsentwicklung}

\begin{tabular}{|c|c|c|c|c|c|}
\hline$S_{o} / S_{u} / f_{p}$ & Probekörper & $E_{s}{ }^{0}$ & $E_{s}^{B}$ & $E_{s}^{B} / E_{s}{ }^{0}$ & $\begin{array}{c}\text { Mittelwert } \\
E_{s}{ }^{B} / E_{s}{ }^{0}\end{array}$ \\
\hline & & $\mathrm{N} / \mathrm{mm}^{2}$ & $\mathrm{~N} / \mathrm{mm}^{2}$ & - & - \\
\hline \multirow{5}{*}{$0,80 / 0,05 / 10$} & PK 001 & 25.435 & 19.895 & 0,78 & \multirow{5}{*}{0,79} \\
\hline & PK 002 & 24.549 & 17.909 & 0,73 & \\
\hline & PK 11 & 24.298 & 19.359 & 0,80 & \\
\hline & PK 10 & 24.414 & 19.649 & 0,80 & \\
\hline & PK 20 & 21.488 & 17.543 & 0,82 & \\
\hline \multirow{9}{*}{$0,70 / 0,05 / 10$} & PK 004 & 24.673 & 17.385 & 0,70 & \multirow{9}{*}{0,73} \\
\hline & PK 14 & 24.804 & 17.840 & 0,72 & \\
\hline & PK 007 & 25.416 & 18.106 & 0,71 & \\
\hline & PK 19 & 23.142 & 17.875 & 0,77 & \\
\hline & PK 26 & 23.411 & 17.435 & 0,74 & \\
\hline & PK 039 & 25.145 & 18.203 & 0,72 & \\
\hline & PK 046 & 24.969 & 19.557 & 0,78 & \\
\hline & PK 22 & 26.101 & 17.992 & 0,69 & \\
\hline & PK 040 & 27.365 & 19.693 & 0,72 & \\
\hline \multirow{8}{*}{$0,60 / 0,05 / 10$} & PK 24 & 24.000 & 17.745 & 0,74 & \multirow{8}{*}{0,66} \\
\hline & PK 21 & 23.511 & 14.014 & 0,60 & \\
\hline & PK 23 & 24.402 & 16.002 & 0,66 & \\
\hline & PK 016 & 25.407 & 18.947 & 0,75 & \\
\hline & PK 13 & 23.337 & 16.649 & 0,71 & \\
\hline & PK 047 & 25.046 & 15.218 & 0,61 & \\
\hline & PK 011 & 26.906 & 15.420 & 0,57 & \\
\hline & PK 043 & 27.688 & 17.408 & 0,63 & \\
\hline \multirow{3}{*}{$0,80 / 0,05 / 1$} & PK 012 & 25.136 & 19.291 & 0,77 & \multirow{3}{*}{0,79} \\
\hline & PK 009 & 24.847 & 20.275 & 0,82 & \\
\hline & PK 014 & 25.062 & 20.040 & 0,80 & \\
\hline \multirow{2}{*}{$0,70 / 0,05 / 1$} & PK 16 & 24.842 & 18.804 & 0,76 & \multirow{2}{*}{0,75} \\
\hline & PK 018 & 25.812 & 19.111 & 0,74 & \\
\hline
\end{tabular}


$S_{o}=0,80 / S_{u}=0,05 / f_{p}=10 \mathrm{~Hz}$

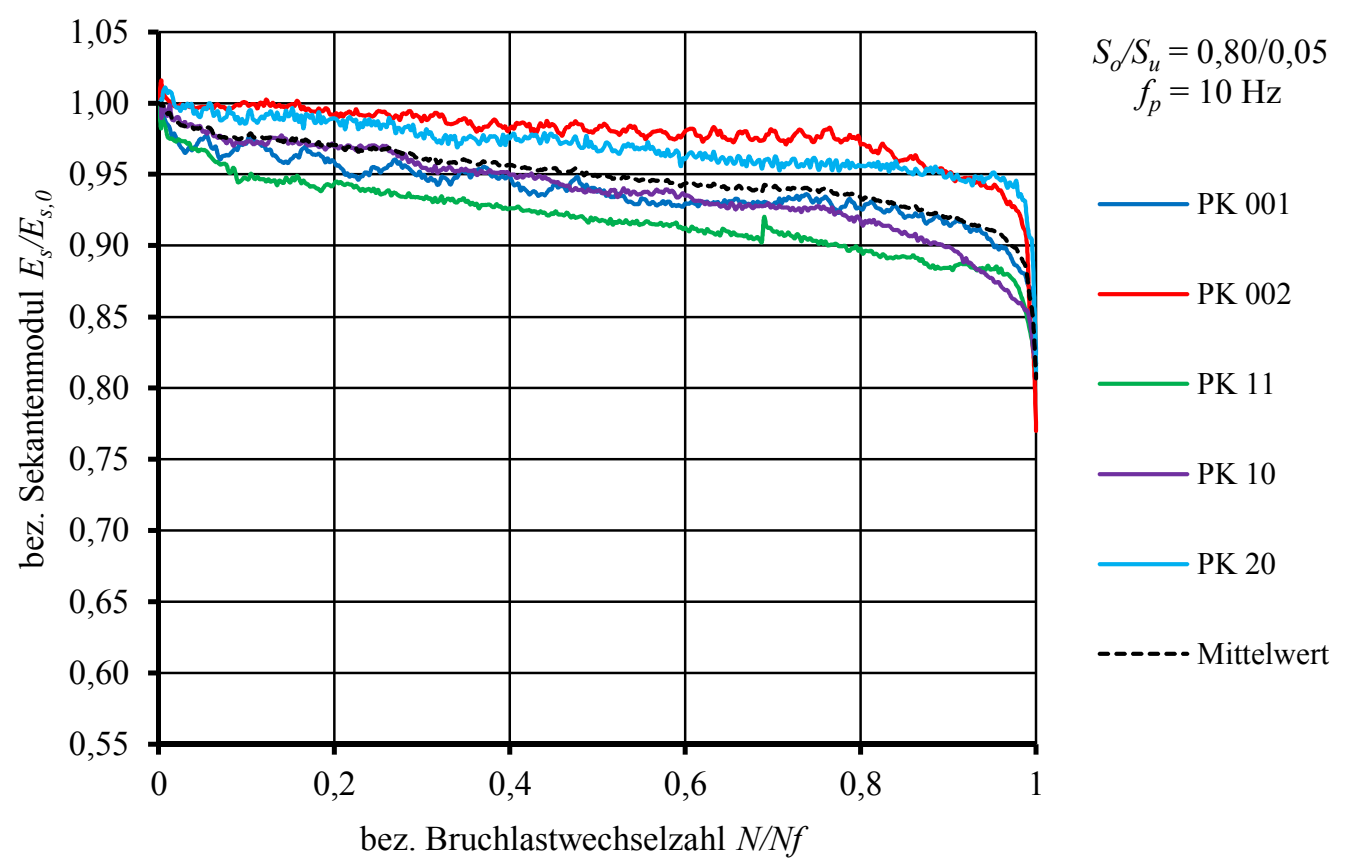

Abbildung A. 2.26: Entwicklung der Sekantensteifigkeit in Abhängigkeit von der bezogenen Bruchlastwechselzahl $S_{o}=0,80 / S_{u}=0,05 / f_{p}=10 \mathrm{~Hz}$

$S_{o}=0,80 / S_{u}=0,05 / f_{p}=1 \mathrm{~Hz}$

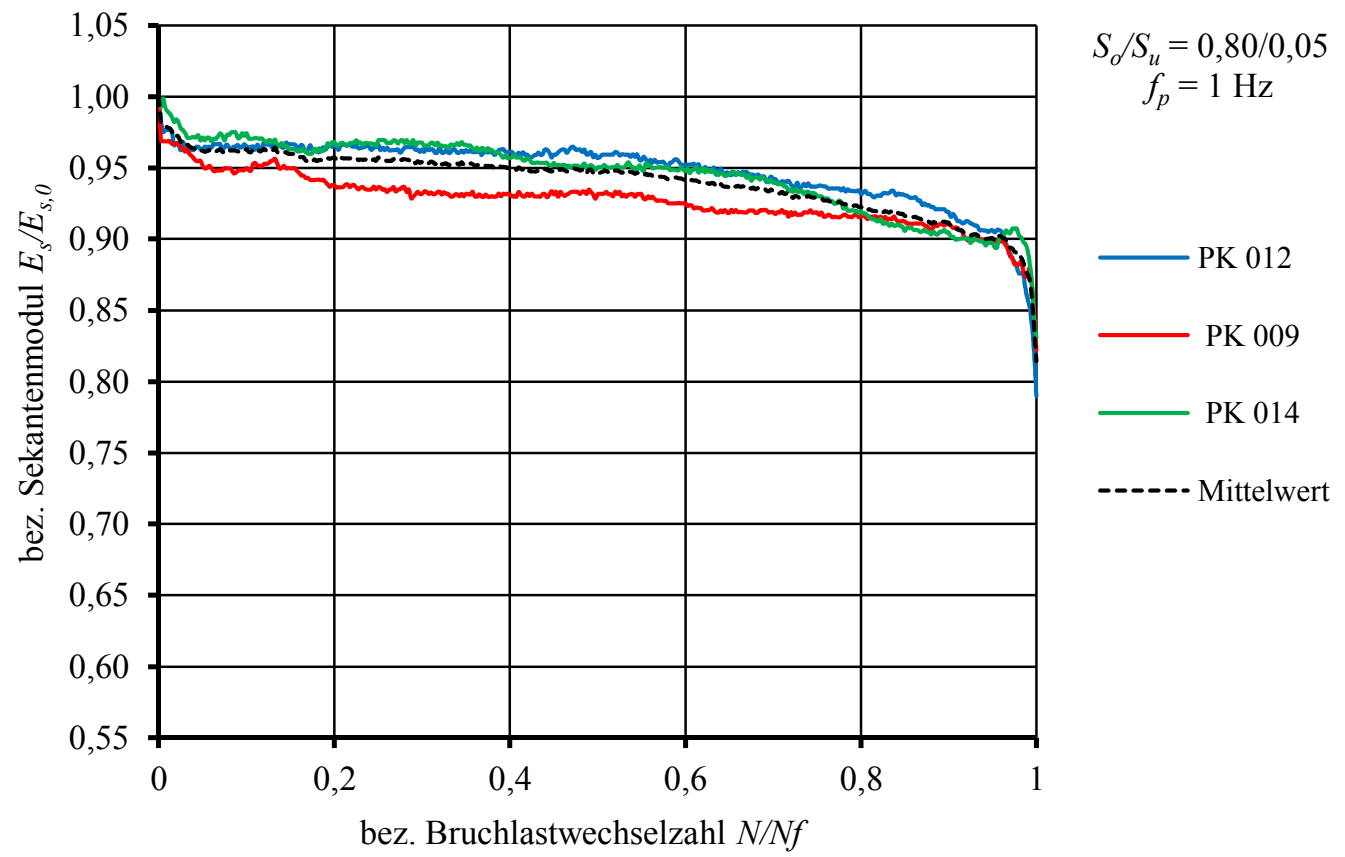

Abbildung A. 2.27: Entwicklung der Sekantensteifigkeit in Abhängigkeit von der bezogenen Bruchlastwechselzahl $S_{o}=0,80 / S_{u}=0,05 / f_{p}=1 \mathrm{~Hz}$ 
$S_{o}=0,70 / S_{u}=0,05 / f_{p}=10 \mathrm{~Hz}$

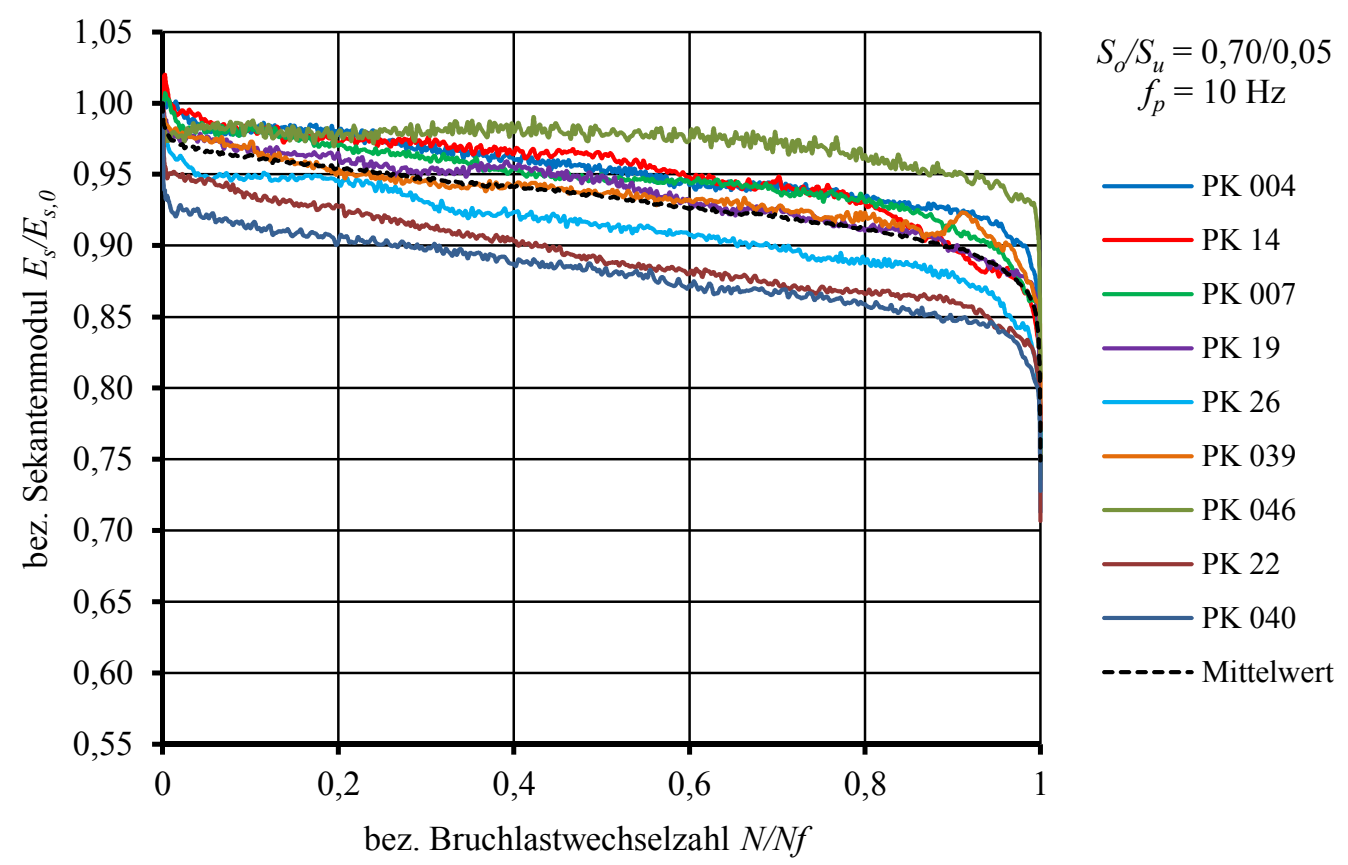

Abbildung A. 2.28: Entwicklung der Sekantensteifigkeit in Abhängigkeit von der bezogenen Bruchlastwechselzahl $S_{o}=0,70 / S_{u}=0,05 / f_{p}=10 \mathrm{~Hz}$

$S_{o}=0,70 / S_{u}=0,05 / f_{p}=1 \mathrm{~Hz}$

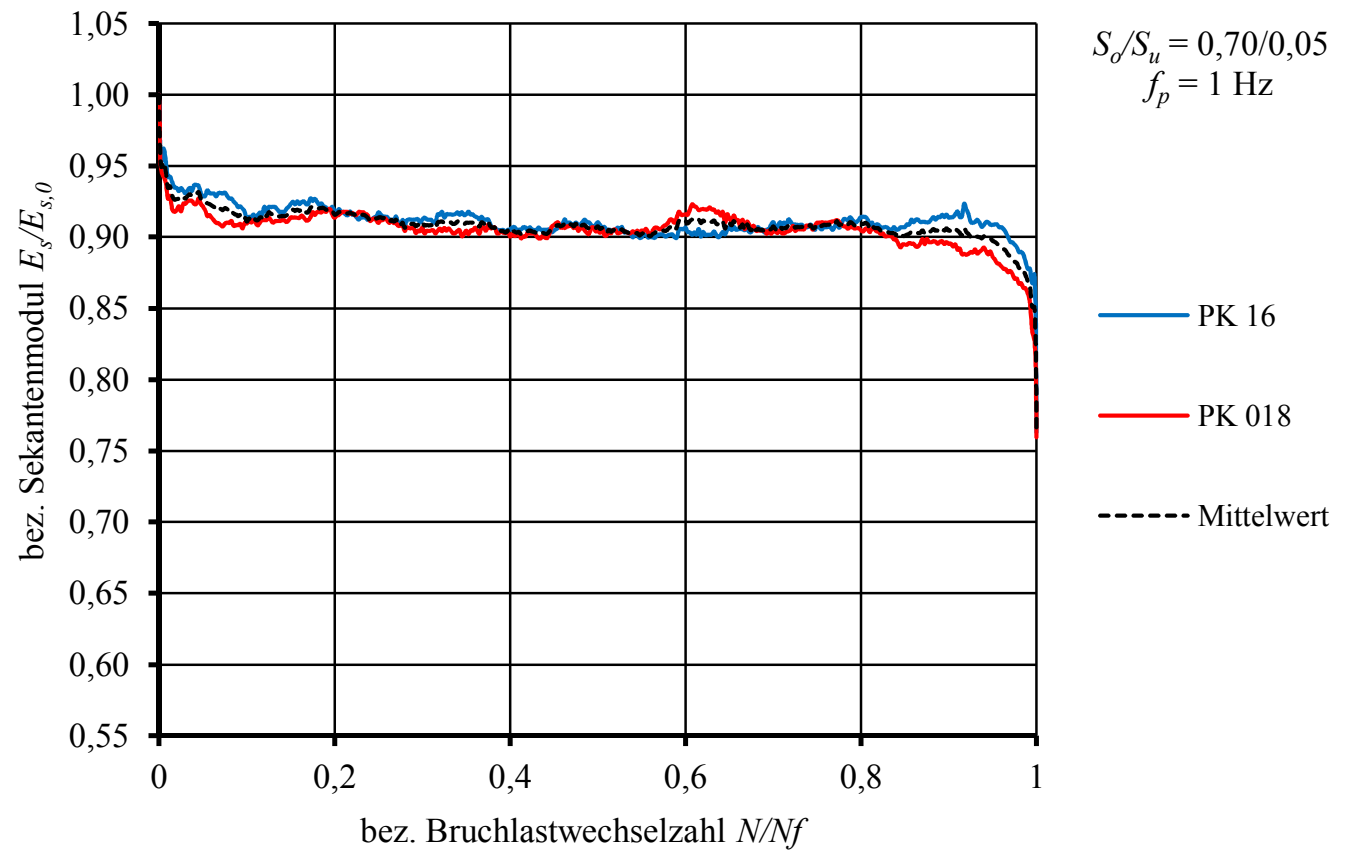

Abbildung A. 2.29: Entwicklung der Sekantensteifigkeit in Abhängigkeit von der bezogenen Bruchlastwechselzahl $S_{o}=0,70 / S_{u}=0,05 / f_{p}=1 \mathrm{~Hz}$ 
$S_{o}=0,60 / S_{u}=0,05 / f_{p}=10 \mathrm{~Hz}$

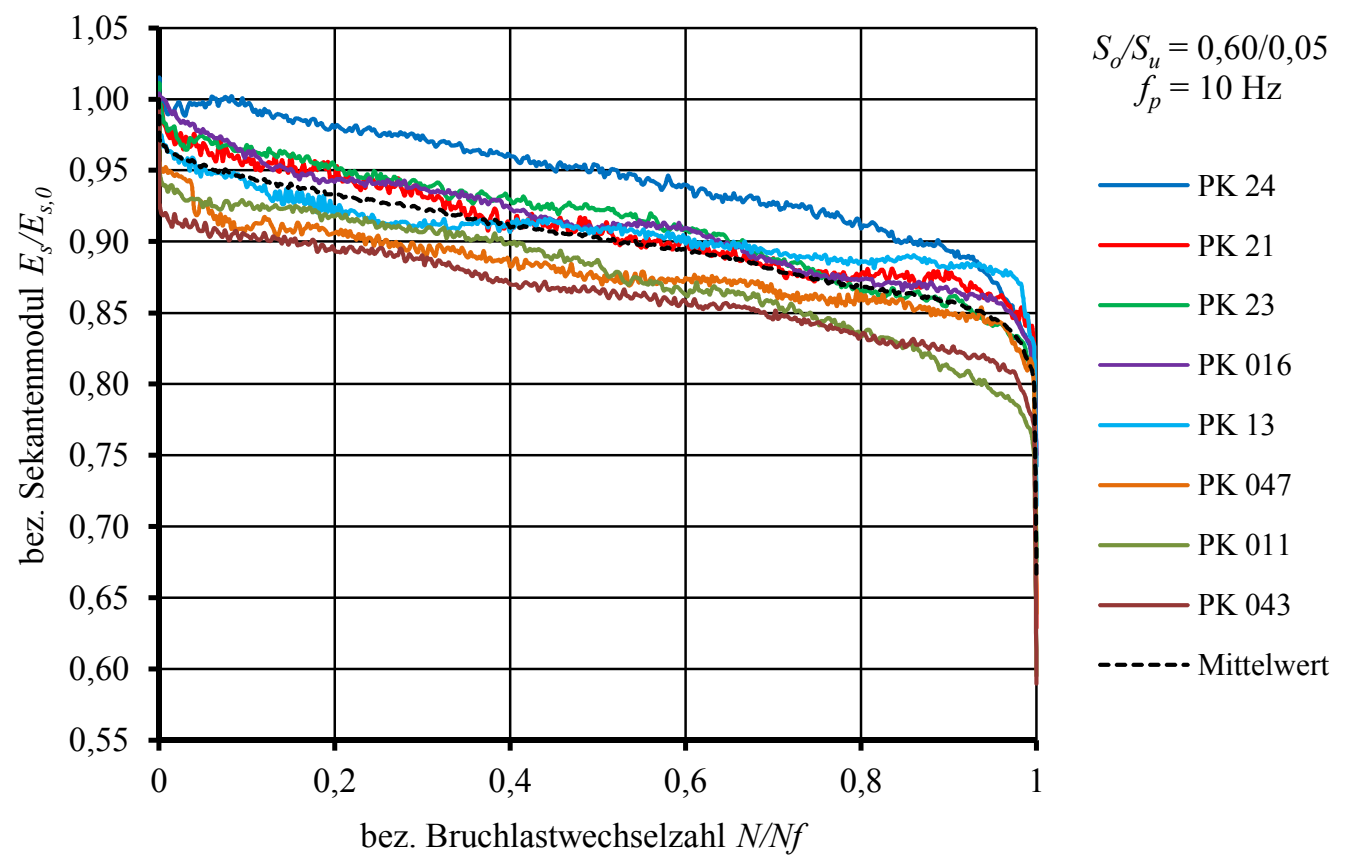

Abbildung A. 2.30: Entwicklung der Sekantensteifigkeit in Abhängigkeit von der bezogenen Bruchlastwechselzahl $S_{o}=0,60 / S_{u}=0,05 / f_{p}=10 \mathrm{~Hz}$ 


\section{A.2.4 Dehnungsentwicklung}

\begin{tabular}{|c|c|c|c|c|c|c|c|}
\hline$S_{o} / S_{u} / f_{p}$ & $\begin{array}{l}\text { Probe- } \\
\text { körper }\end{array}$ & $\log . \bar{\varepsilon}_{\mathbf{o}}{ }^{I I}$ & $\log . \bar{\varepsilon}_{\mathrm{u}} \mathrm{II}$ & $\varepsilon_{o}{ }^{0}$ & $\varepsilon_{o}^{B}$ & $\varepsilon_{e l}{ }^{0}$ & $\varepsilon_{e l}^{B}$ \\
\hline & & $\mathrm{LW}^{-1}$ & $\mathrm{LW}^{-1}$ & $\%$ & $\%$ & $\%$ & $\%$ \\
\hline \multirow{5}{*}{$\begin{array}{c}0,80 / 0,05 / 10 \\
\text { Serie I }\end{array}$} & PK 001 & $-7,110$ & $-7,269$ & $-3,325$ & $-1,020$ & $-2,287$ & $-2,822$ \\
\hline & PK 002 & $-7,194$ & $-7,326$ & $-3,666$ & $-1,048$ & $-2,287$ & $-3,134$ \\
\hline & PK 11 & $-6,747$ & $-6,875$ & $-3,634$ & $-1,196$ & $-2,310$ & $-2,900$ \\
\hline & PK 10 & $-6,315$ & $-6,573$ & $-3,311$ & $-0,910$ & $-2,299$ & $-2,857$ \\
\hline & PK 20 & $-7,124$ & $-7,271$ & $-3,981$ & $-1,309$ & $-2,612$ & $-3,200$ \\
\hline \multirow{4}{*}{$\begin{array}{c}0,70 / 0,05 / 10 \\
\text { Serie I }\end{array}$} & PK 004 & $-7,930$ & $-8,144$ & $-3,543$ & $-1,210$ & $-1,991$ & $-2,825$ \\
\hline & PK 14 & $-8,002$ & $-8,173$ & $-3,525$ & $-1,280$ & $-1,980$ & $-2,753$ \\
\hline & PK 007 & $-7,945$ & $-8,104$ & $-3,243$ & $-1,000$ & $-1,933$ & $-2,713$ \\
\hline & PK 19 & $-7,874$ & $-8,081$ & $-3,313$ & $-1,032$ & $-2,123$ & $-2,748$ \\
\hline \multirow{4}{*}{$\begin{array}{c}0,60 / 0,05 / 10 \\
\text { Serie I }\end{array}$} & PK 24 & $-8,226$ & $-8,396$ & $-3,266$ & $-1,317$ & $-1,754$ & $-2,373$ \\
\hline & PK 21* & $-8,298$ & $-8,363$ & $-4,534$ & $-2,112$ & $-1,791$ & $-3,004$ \\
\hline & PK 23 & $-8,124$ & $-8,252$ & $-3,634$ & $-1,491$ & $-1,725$ & $-2,631$ \\
\hline & PK 016 & $-8,305$ & $-8,441$ & $-3,139$ & $-1,324$ & $-1,657$ & $-2,222$ \\
\hline \multirow{3}{*}{$\begin{array}{c}0,80 / 0,05 / 1 \\
\text { Serie I }\end{array}$} & PK 012 & $-6,839$ & $-6,984$ & $-3,650$ & $-1,197$ & $-2,233$ & $-2,910$ \\
\hline & PK 009 & $-6,780$ & $-6,871$ & $-3,525$ & $-1,194$ & $-2,259$ & $-2,769$ \\
\hline & PK 014 & $-6,424$ & $-6,585$ & $-3,464$ & $-1,103$ & $-2,240$ & $-2,801$ \\
\hline \multirow{2}{*}{$\begin{array}{c}0,70 / 0,05 / 1 \\
\text { Serie I }\end{array}$} & PK 16 & $-8,043$ & $-8,064$ & $-4,313$ & $-2,136$ & $-1,977$ & $-2,612$ \\
\hline & PK 018 & $-8,185$ & $-8,173$ & $-4,128$ & $-1,982$ & $-1,903$ & $-2,570$ \\
\hline \multirow{5}{*}{$\begin{array}{c}0,80 / 0,05 / 10 \\
\text { Serie II }\end{array}$} & PK 26 & $-8,067$ & $-8,300$ & $-3,648$ & $-1,327$ & $-2,098$ & $-2,817$ \\
\hline & PK 039 & $-8,194$ & $-8,365$ & $-3,366$ & $-1,154$ & $-1,953$ & $-2,698$ \\
\hline & PK 046 & $-8,121$ & $-8,176$ & $-3,183$ & $-1,108$ & $-1,967$ & $-2,512$ \\
\hline & PK 22 & $-8,090$ & $-8,537$ & $-3,211$ & $-1,025$ & $-1,882$ & $-2,730$ \\
\hline & PK 040 & $-8,149$ & $-8,457$ & $-2,970$ & $-0,906$ & $-1,795$ & $-2,494$ \\
\hline \multirow{4}{*}{$\begin{array}{c}0,70 / 0,05 / 10 \\
\text { Serie II }\end{array}$} & PK 13 & $-8,490$ & $-8,541$ & $-3,638$ & $-1,546$ & $-1,804$ & $-2,529$ \\
\hline & PK 047 & $-8,885$ & $-8,959$ & $-3,926$ & $-1,671$ & $-1,681$ & $-2,767$ \\
\hline & PK 011 & $-8,538$ & $-8,739$ & $-3,449$ & $-1,333$ & $-1,565$ & $-2,730$ \\
\hline & PK 043 & $-8,744$ & $-8,896$ & $-3,583$ & $-1,786$ & $-1,521$ & $-2,419$ \\
\hline
\end{tabular}

* Der Probekörper PK 21 weist im Vergleich zu den übrigen Probekörpern der Beanspruchungsgruppe ein ungewöhnliches Dehnungsverhalten auf, vgl. Abbildung A. 2.36. Die Dehnungen wachsen im Vergleich zu denen der übrigen Versuchskörper übermäßig an. Die Ursache ist unbekannt. Der Versuchskörper wurde als Ausreißer gewertet und nicht weiter betrachtet. 
$S_{o}=0,80 / S_{u}=0,05 / f_{p}=10 \mathrm{~Hz}-$ Serie I

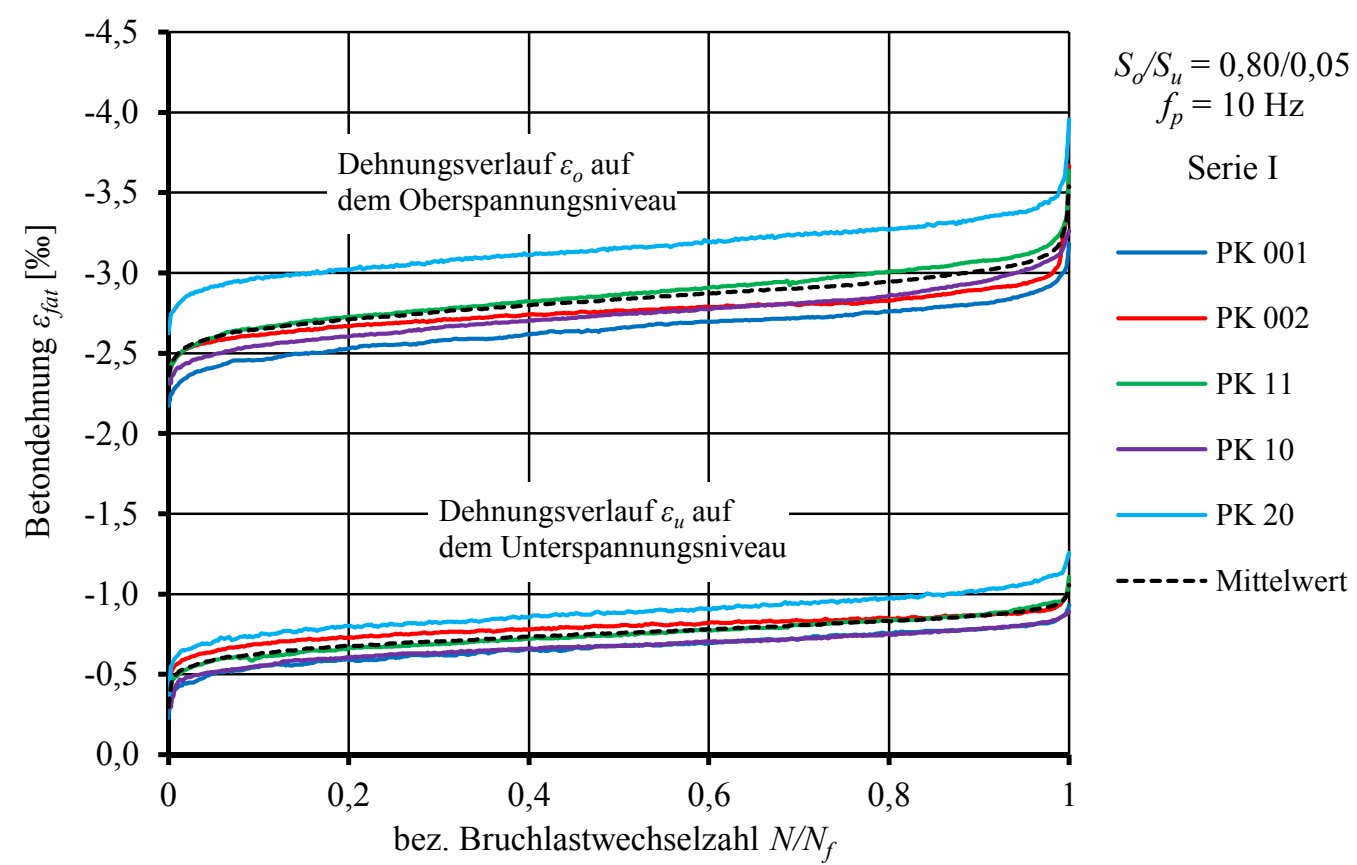

Abbildung A. 2.31: Dehnungsentwicklung auf dem Ober- und Unterspannungsniveau Serie I, $S_{o}=0,80 / S_{u}=0,05 / f_{p}=10 \mathrm{~Hz}$

$S_{o}=0,80 / S_{u}=0,05 / f_{p}=1 \mathrm{~Hz}-$ Serie I

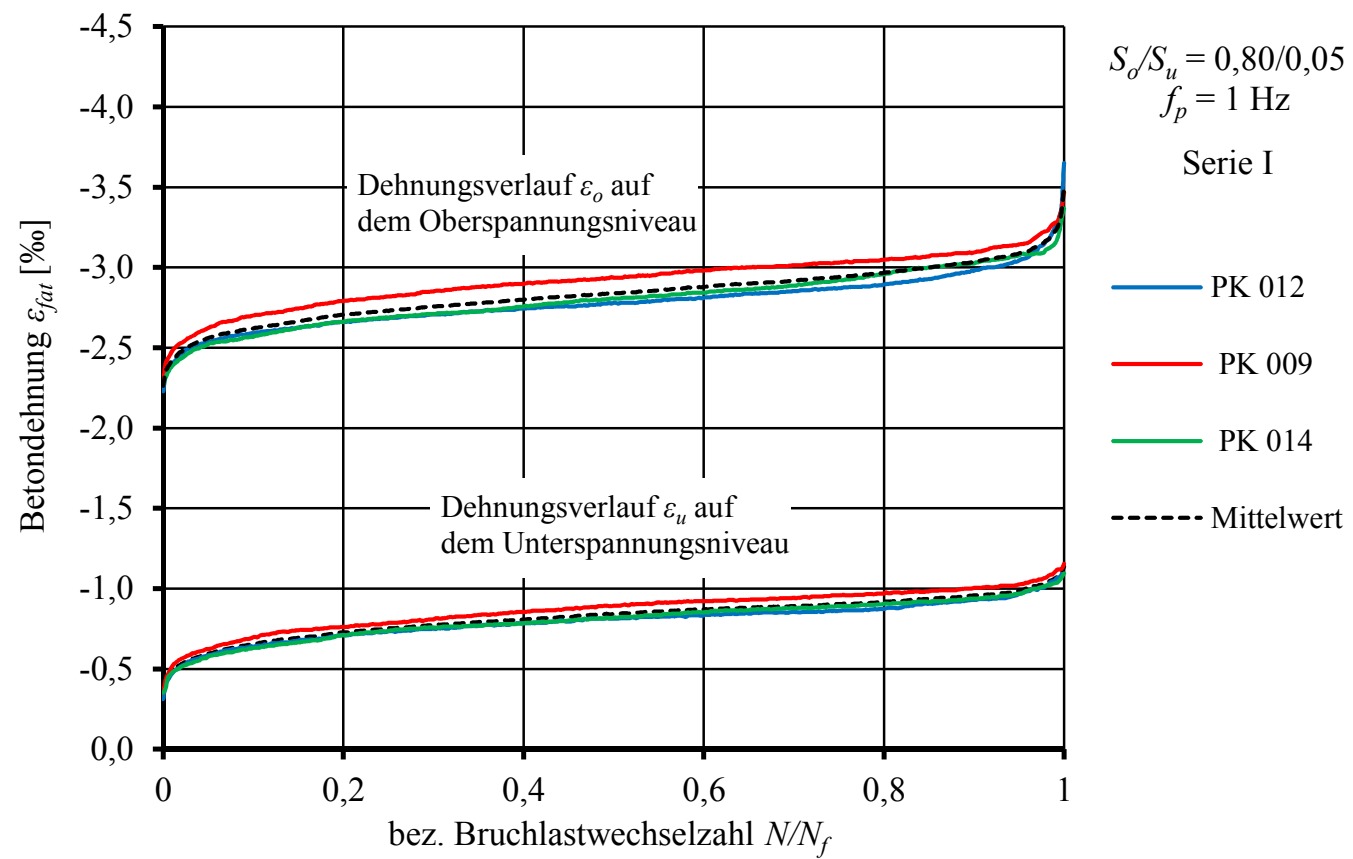

Abbildung A. 2.32: Dehnungsentwicklung auf dem Ober- und Unterspannungsniveau Serie I, $S_{o}=0,80 / S_{u}=0,05 / f_{p}=1 \mathrm{~Hz}$ 
$S_{o}=0,70 / S_{u}=0,05 / f_{p}=10 \mathrm{~Hz}-$ Serie I

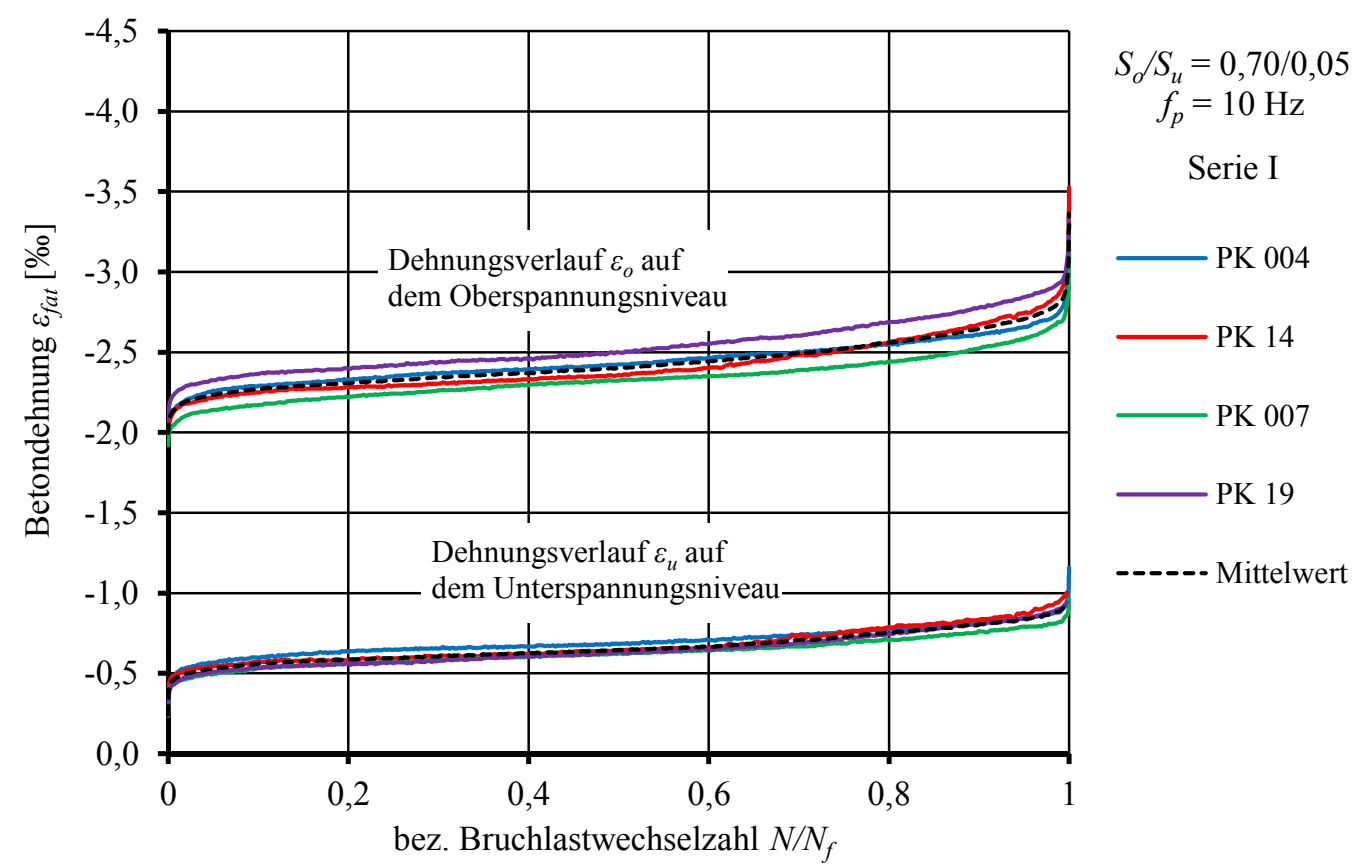

Abbildung A. 2.33: Dehnungsentwicklung auf dem Ober- und Unterspannungsniveau Serie I, $S_{o}=0,70 / S_{u}=0,05 / f_{p}=10 \mathrm{~Hz}$

$S_{o}=0,70 / S_{u}=0,05 / f_{p}=10 \mathrm{~Hz}-$ Serie II

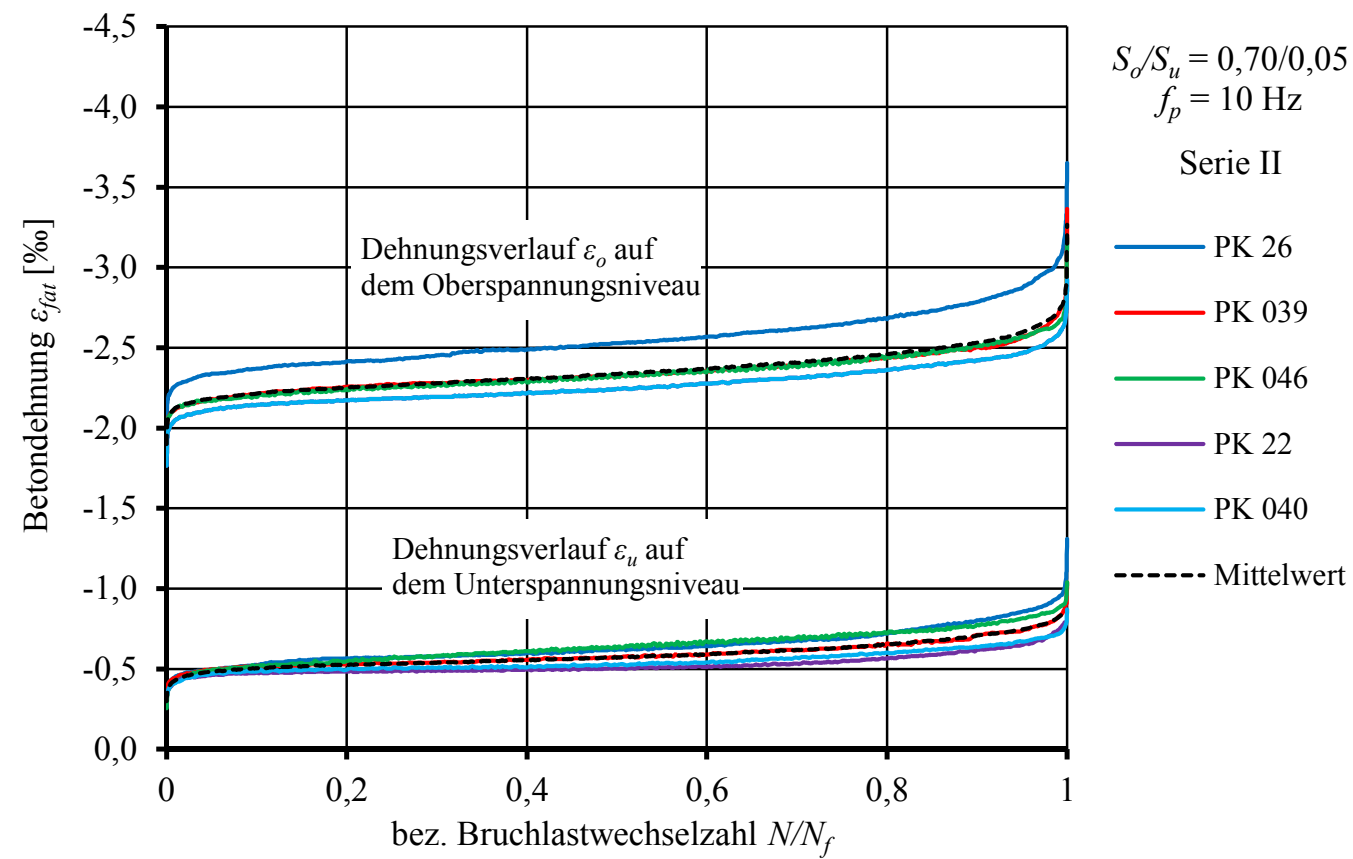

Abbildung A. 2.34: Dehnungsentwicklung auf dem Ober- und Unterspannungsniveau Serie II, $S_{o}=0,70 / S_{u}=0,05 / f_{p}=10 \mathrm{~Hz}$ 
$S_{o}=0,70 / S_{u}=0,05 / f_{p}=1 \mathrm{~Hz}-$ Serie I

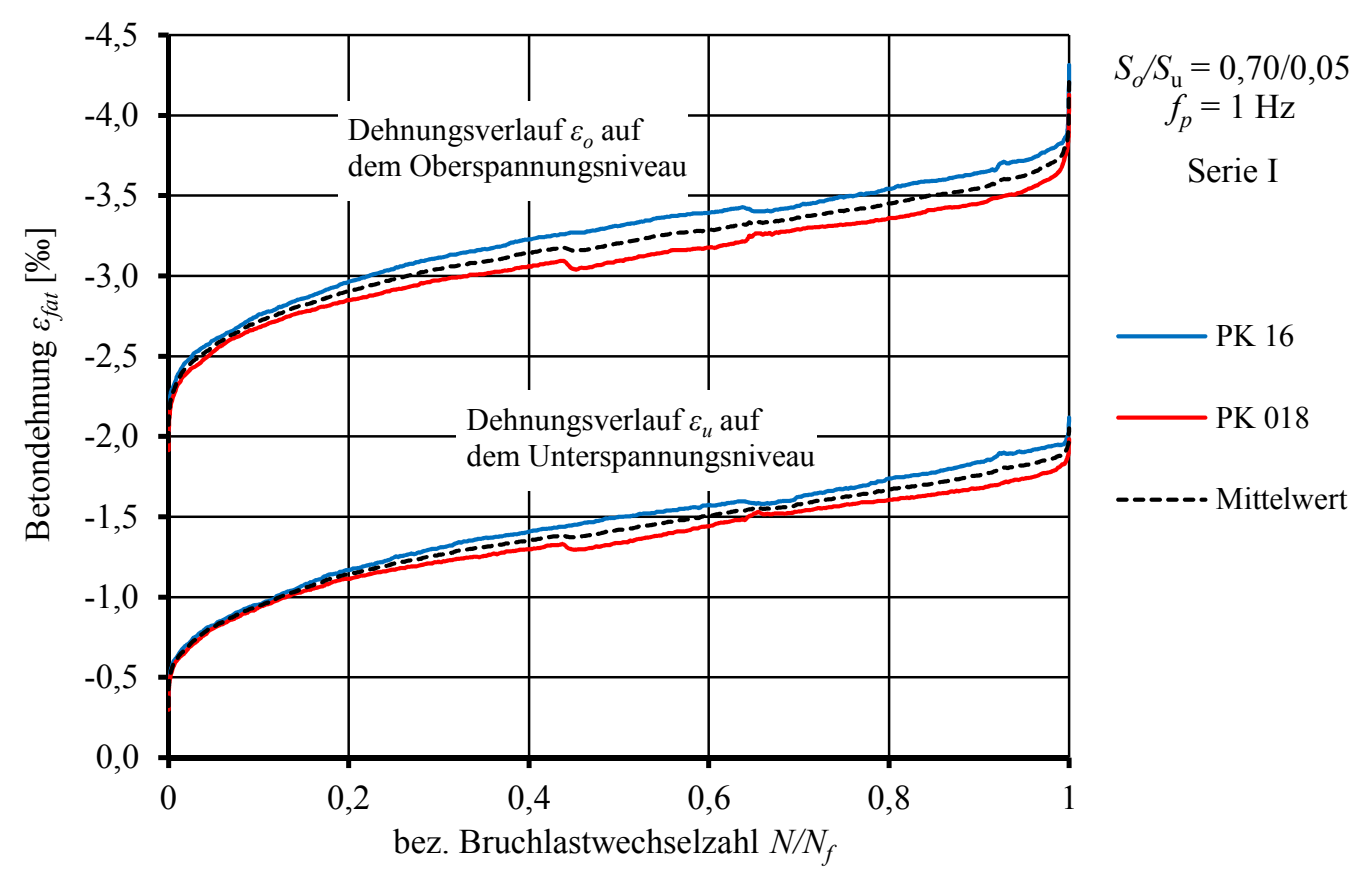

Abbildung A. 2.35: Dehnungsentwicklung auf dem Ober- und Unterspannungsniveau Serie I, $S_{o}=0,70 / S_{u}=0,05 / f_{p}=1 \mathrm{~Hz}$ 
$S_{o}=0,60 / S_{u}=0,05 / f_{p}=10 \mathrm{~Hz}-$ Serie I

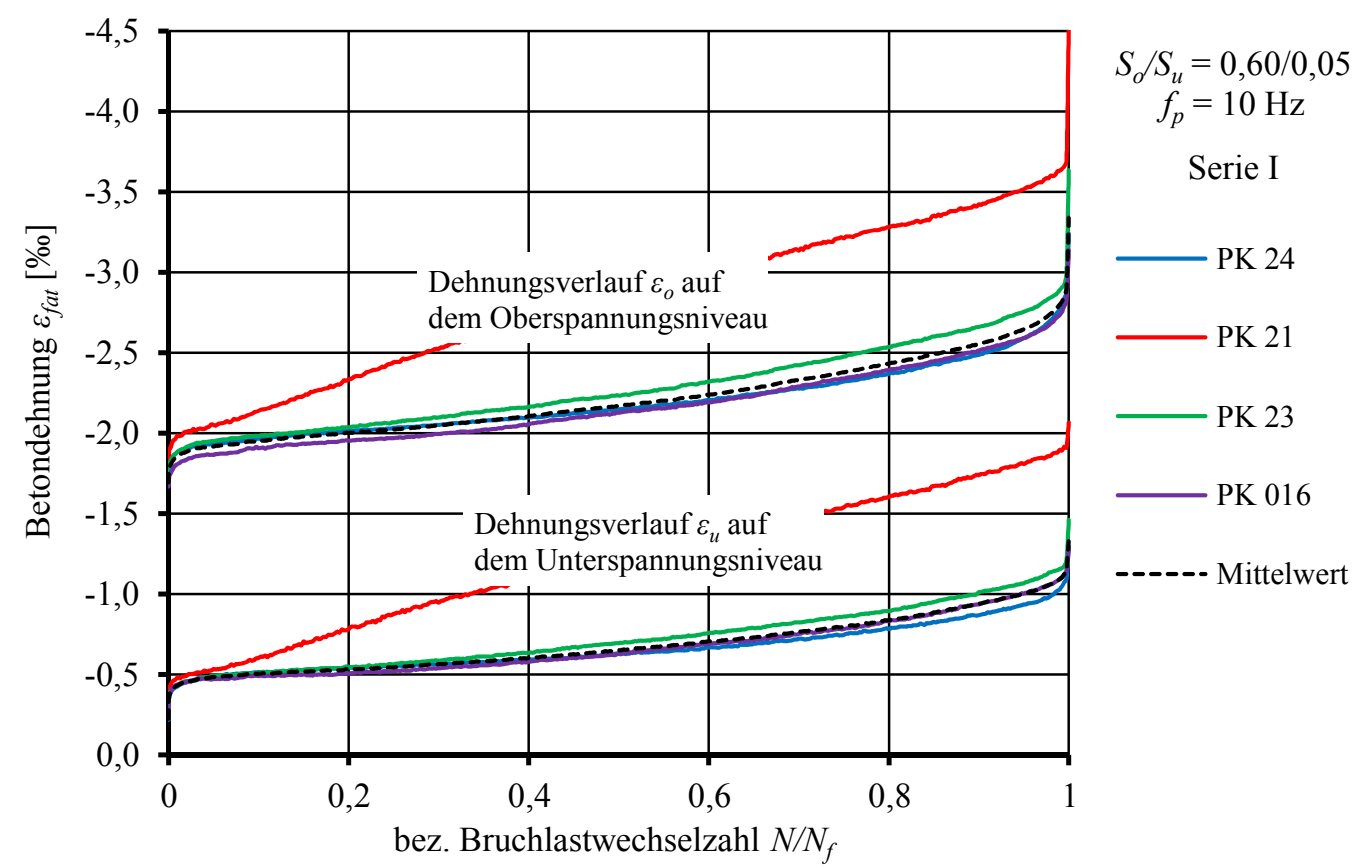

Abbildung A. 2.36: Dehnungsentwicklung auf dem Ober- und Unterspannungsniveau Serie I, $S_{o}=0,60 / S_{u}=0,05 / f_{p}=10 \mathrm{~Hz}$

$S_{o}=0,60 / S_{u}=0,05 / f_{p}=10 \mathrm{~Hz}-$ Serie II

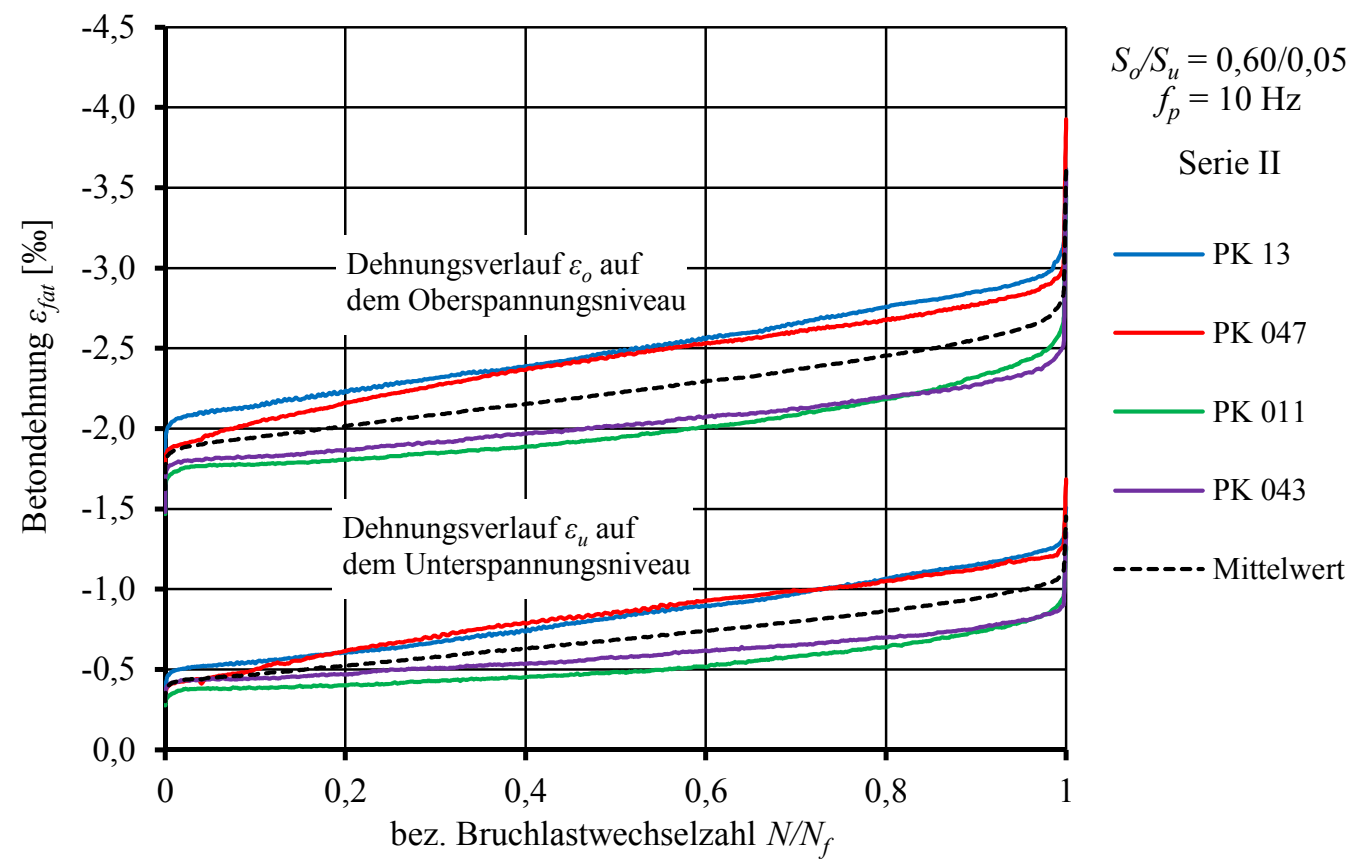

Abbildung A. 2.37: Dehnungsentwicklung auf dem Ober- und Unterspannungsniveau Serie II, $S_{o}=0,60 / S_{u}=0,05 / f_{p}=10 \mathrm{~Hz}$ 


\section{A.2.5 Ermüdungsbruchdehnungen in Abhängigkeit der Bruchlastwechselzahl}

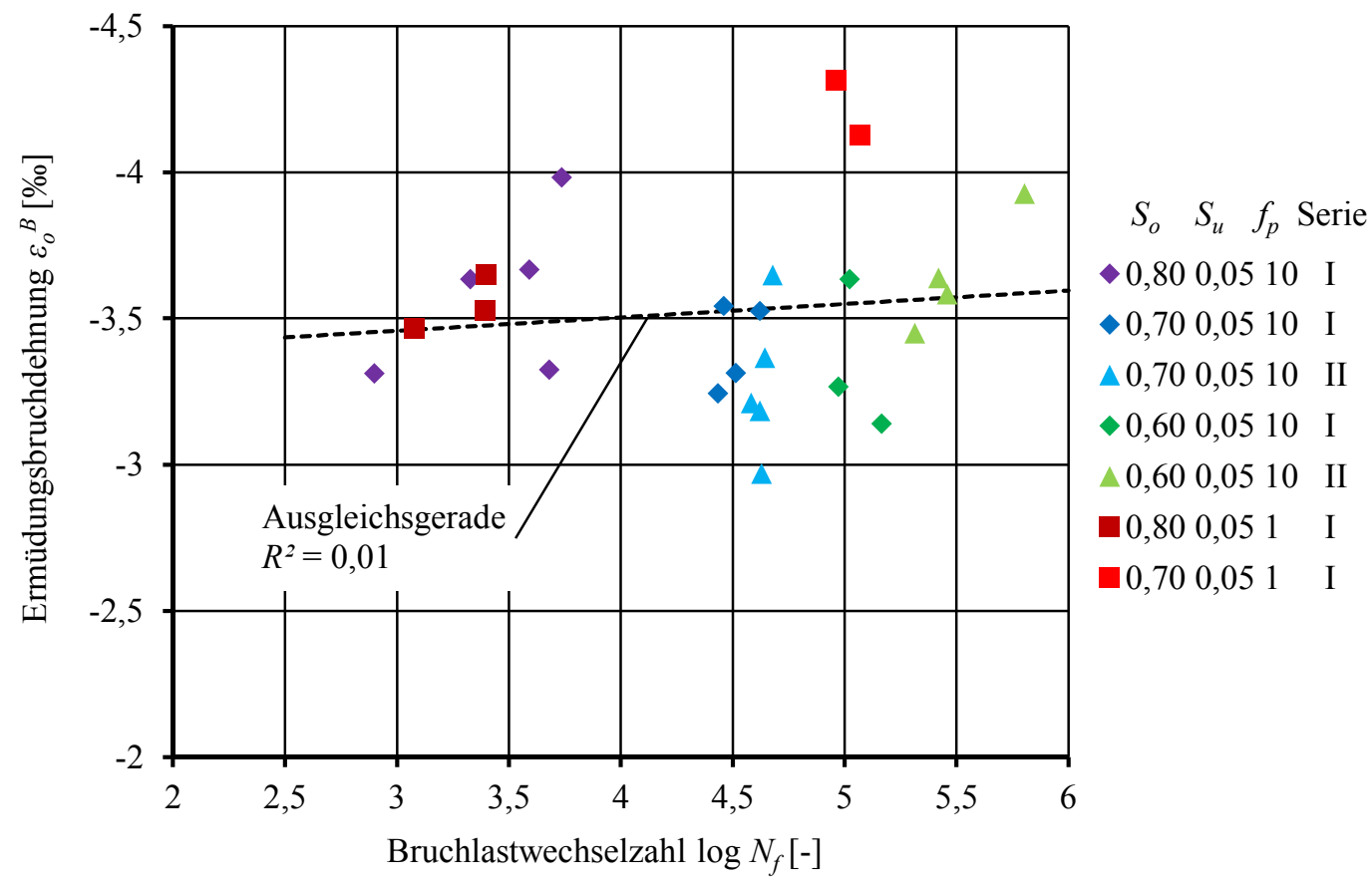

Abbildung A. 2.38: Ermüdungsbruchdehnungen auf dem Oberspannungsniveau in Abhängigkeit der logarithmierten Bruchlastwechselzahl

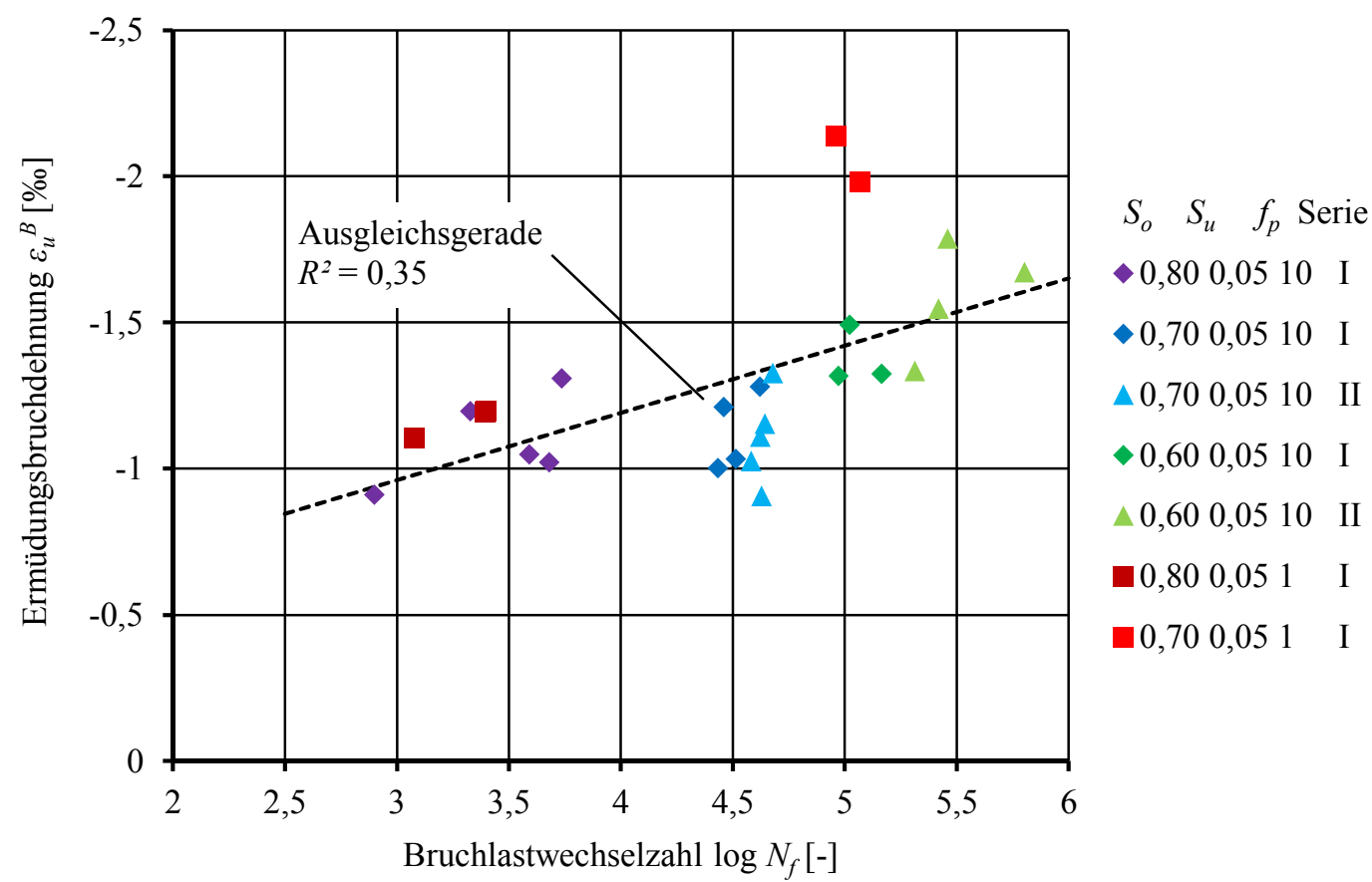

Abbildung A. 2.39: Ermüdungsbruchdehnungen auf dem Unterspannungsniveau in Abhängigkeit der logarithmierten Bruchlastwechselzahl 


\section{A.3 Kriechkurven}

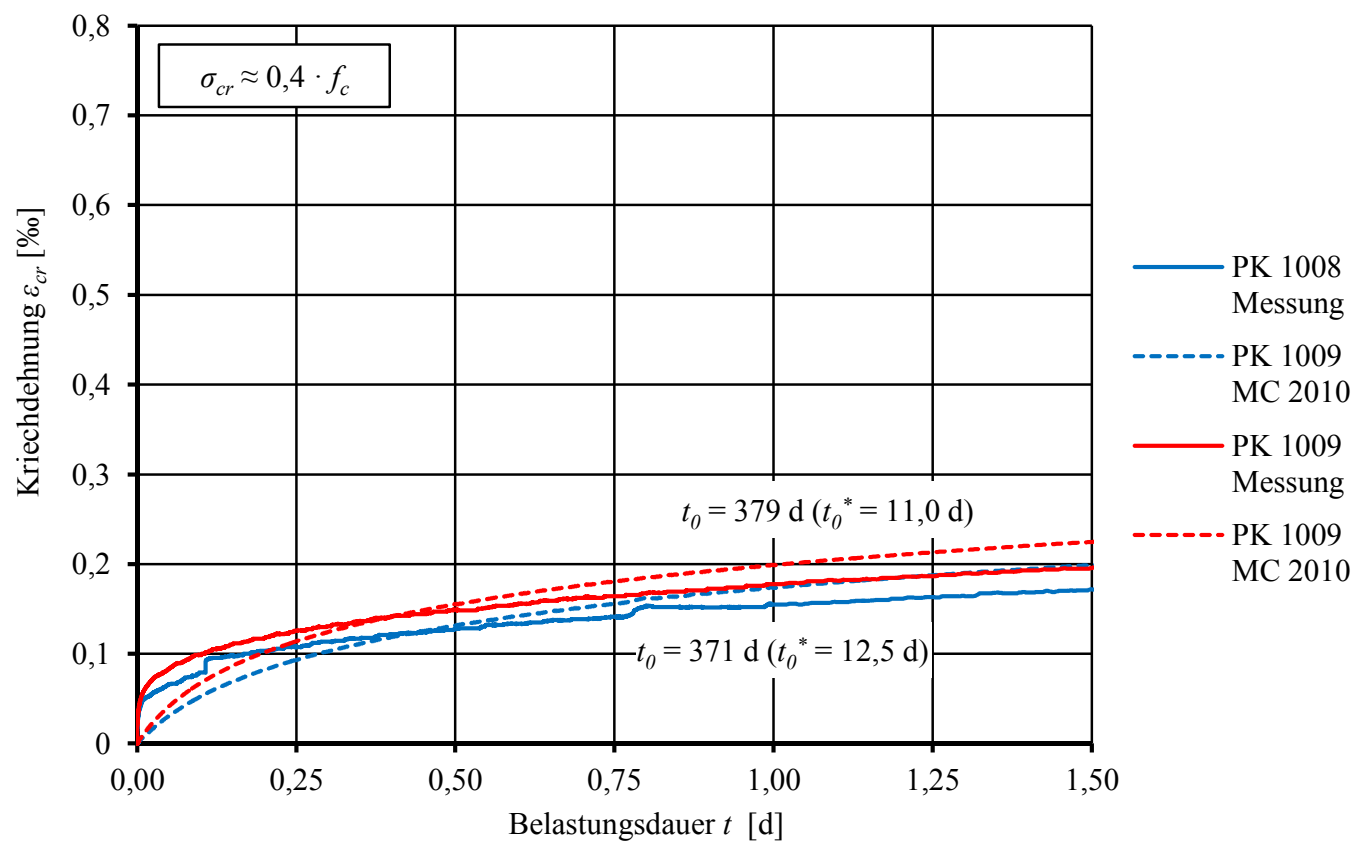

Abbildung A. 3.1: Experimentelle und rechnerische Kriechverläufe für $\sigma_{c r}=0,4 \cdot f_{c}$

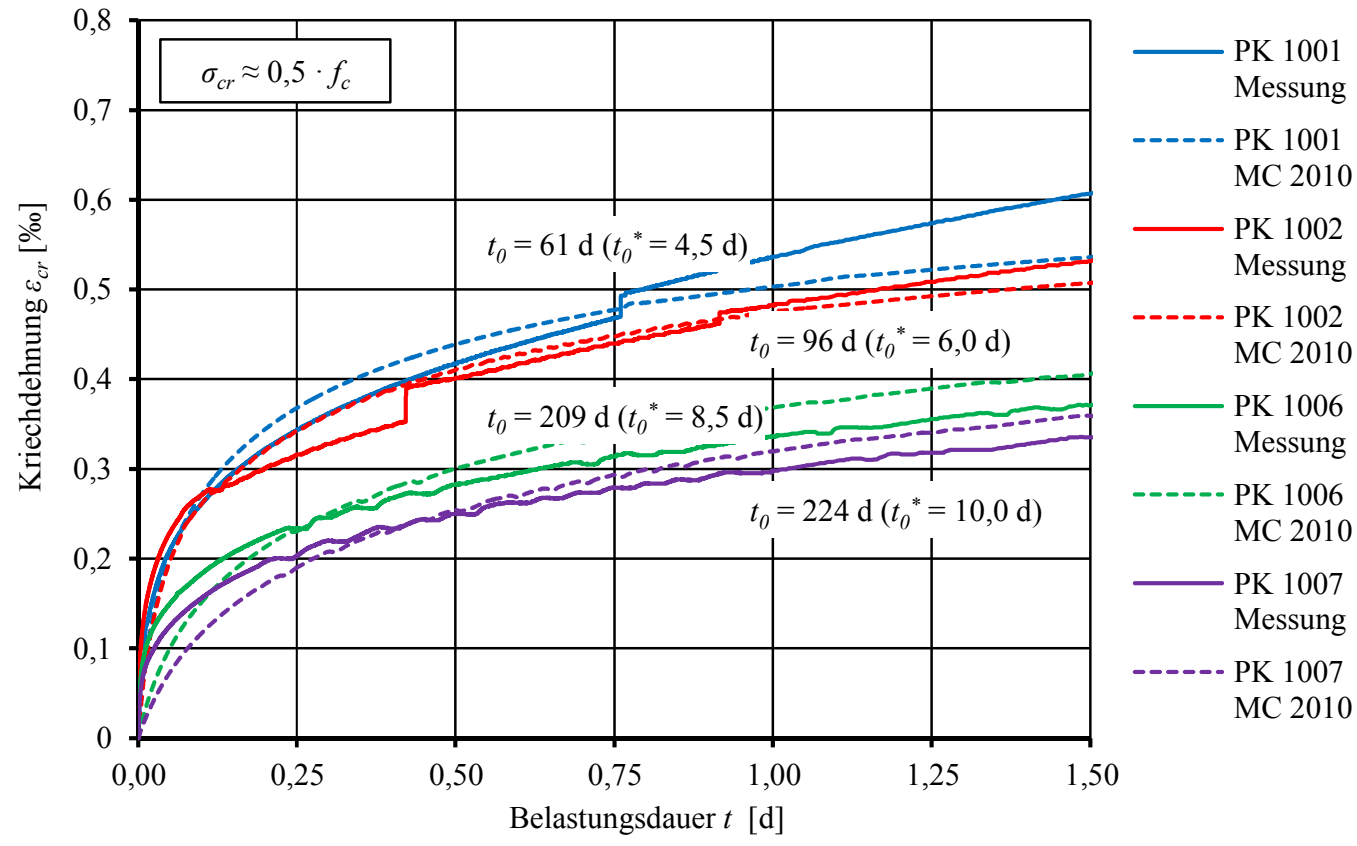

Abbildung A. 3.2: Experimentelle und rechnerische Kriechverläufe für $\sigma_{c r}=0,5 \cdot f_{c}$ 


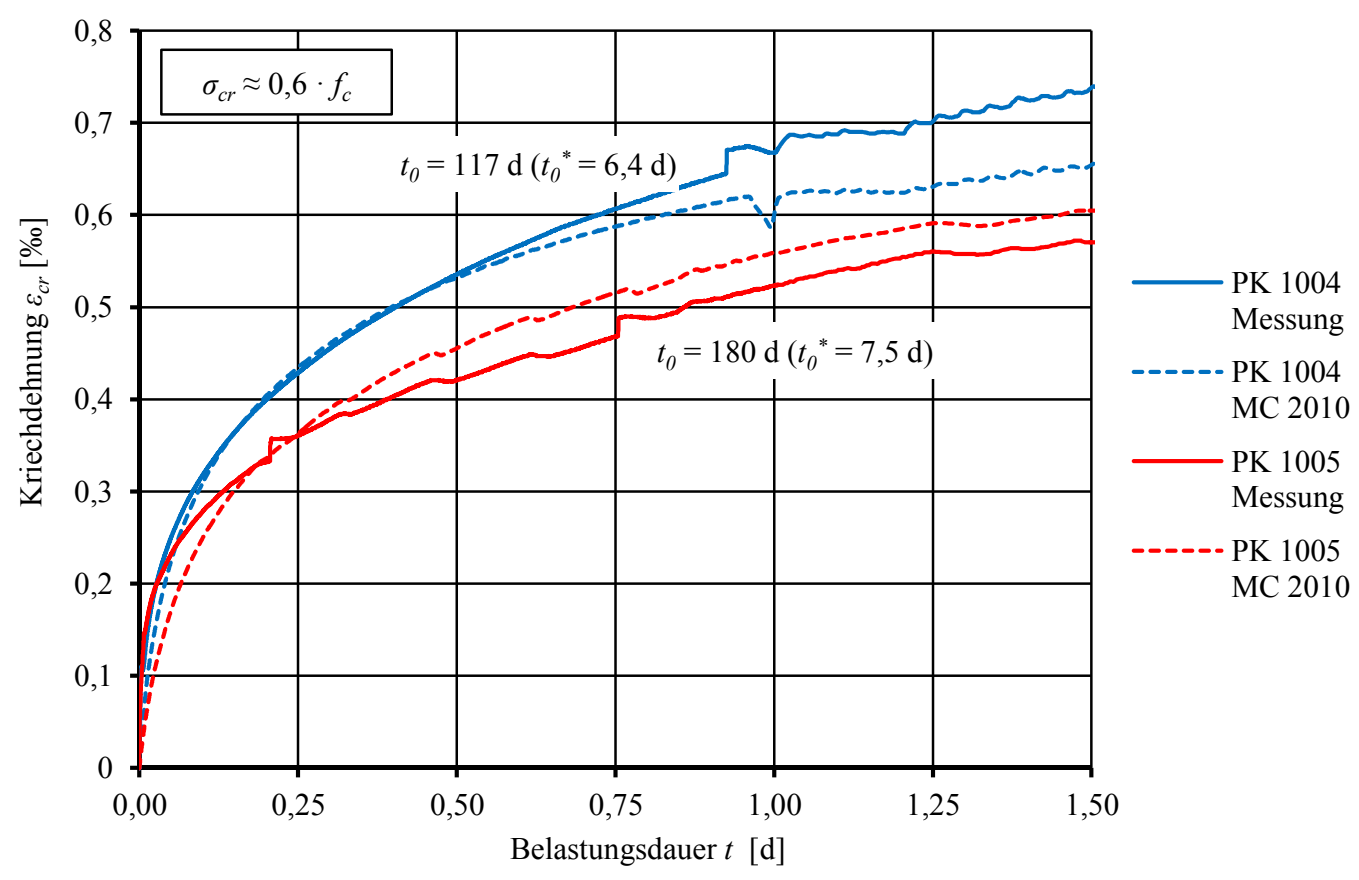

Abbildung A. 3.3: Experimentelle und rechnerische Kriechverläufe für $\sigma_{c r}=0,6 \cdot f_{c}$ 


\section{A.4 Dehnungsanteile im Ermüdungsversuch}

$S_{o}=0,80 / S_{u}=0,05 / f_{p}=10 \mathrm{~Hz}-$ Serie I

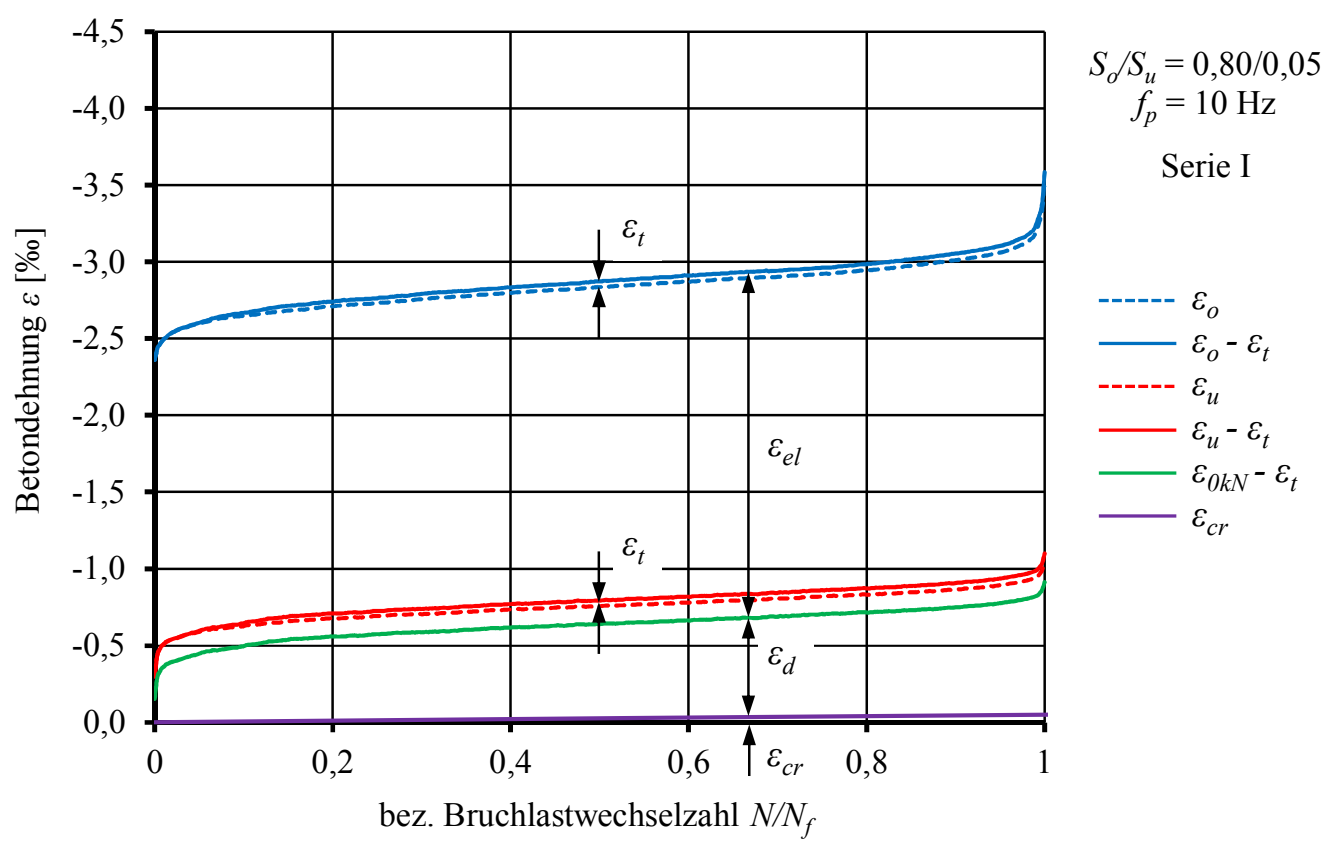

Abbildung A. 4.1: Gemittelte Dehnungsanteile in Abhängigkeit von der bezogenen Bruchlastwechselzahl Serie I, $S_{o}=0,80 / S_{u}=0,05 / f_{p}=10 \mathrm{~Hz}$

$S_{o}=0,80 / S_{u}=0,05 / f_{p}=1 \mathrm{~Hz}-$ Serie I

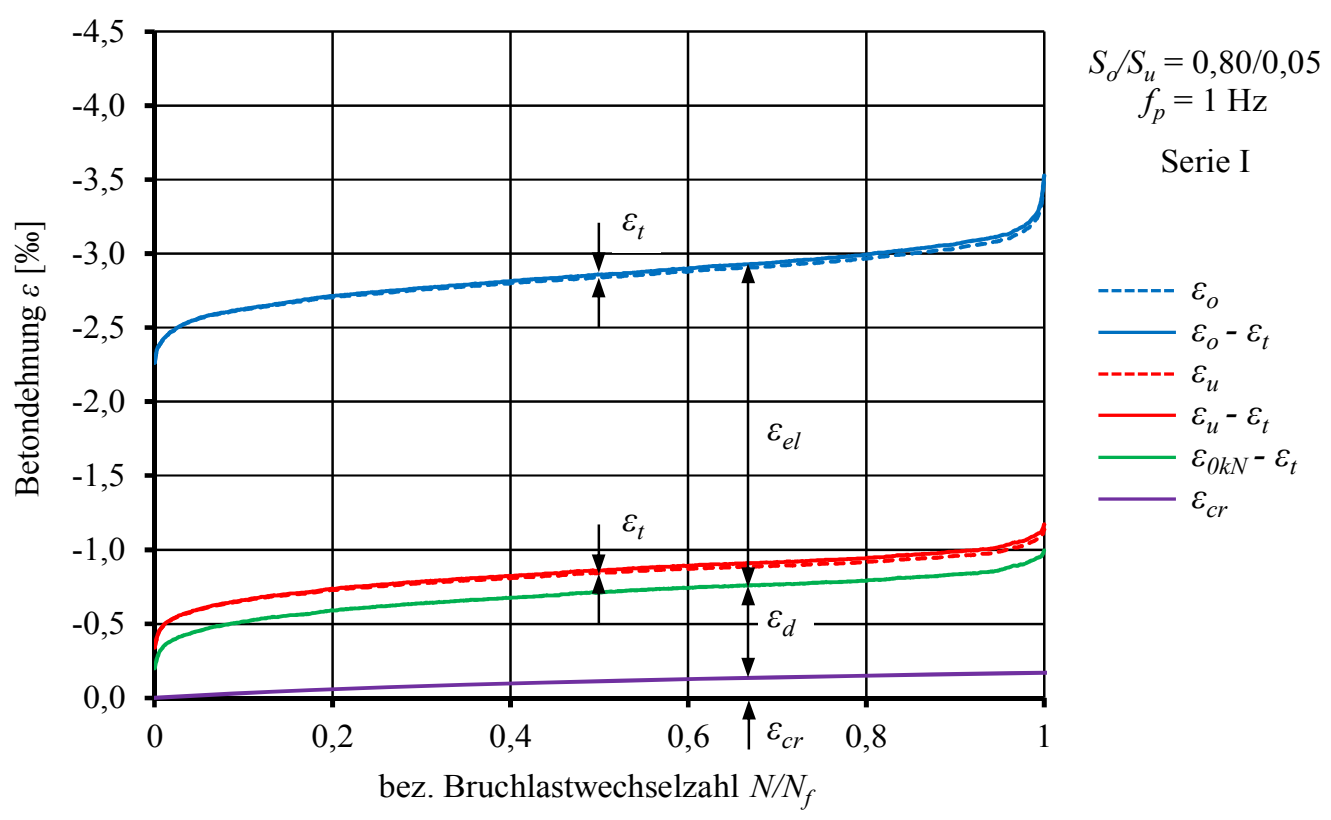

Abbildung A. 4.2: Gemittelte Dehnungsanteile in Abhängigkeit von der bezogenen Bruchlastwechselzahl Serie I, $S_{o}=0,80 / S_{u}=0,05 / f_{p}=1 \mathrm{~Hz}$ 
$S_{o}=0,70 / S_{u}=0,05 / f_{p}=10 \mathrm{~Hz}-$ Serie I

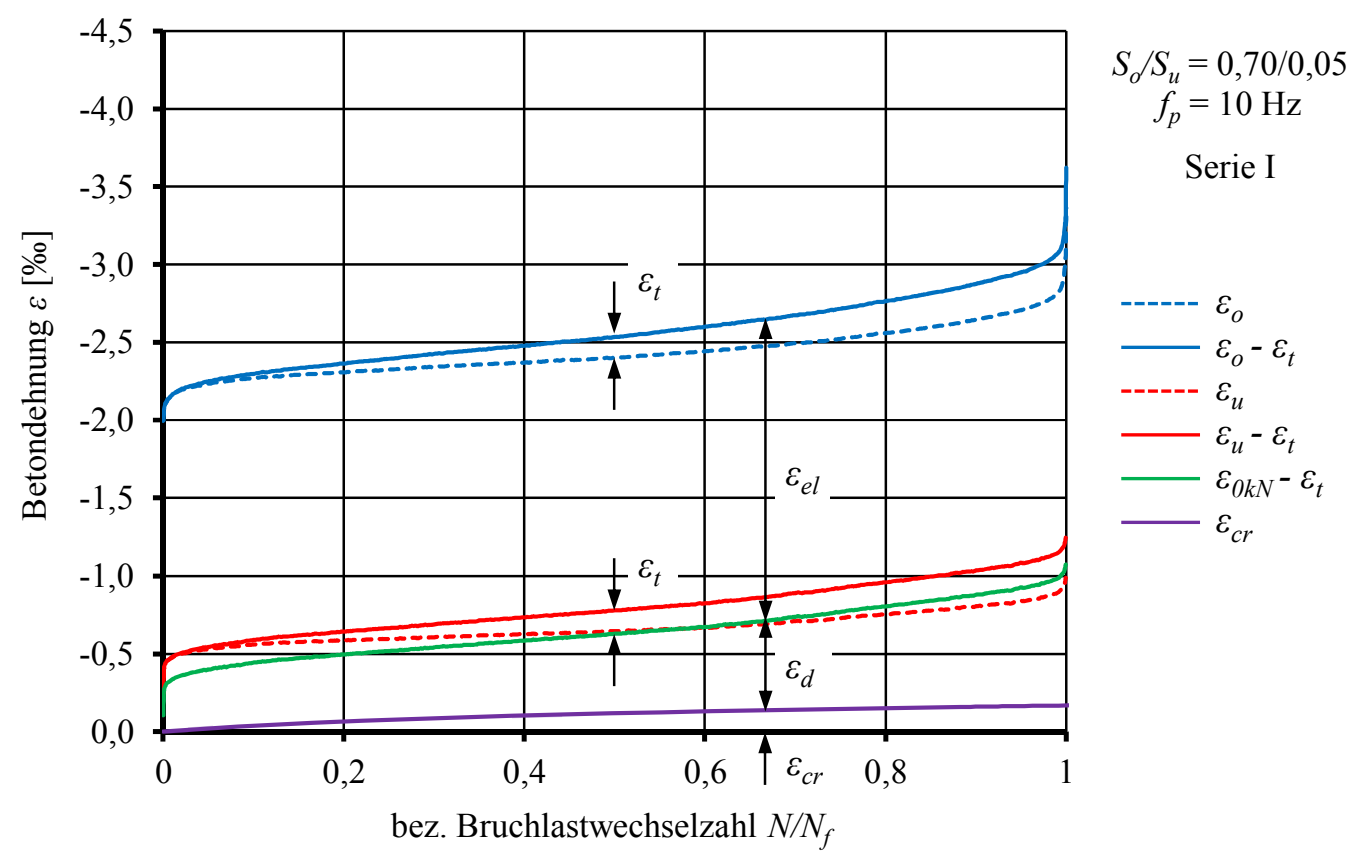

Abbildung A. 4.3: Gemittelte Dehnungsanteile in Abhängigkeit von der bezogenen Bruchlastwechselzahl Serie I, $S_{o}=0,70 / S_{u}=0,05 / f_{p}=10 \mathrm{~Hz}$

$S_{o}=0,70 / S_{u}=0,05 / f_{p}=10 \mathrm{~Hz}-$ Serie II

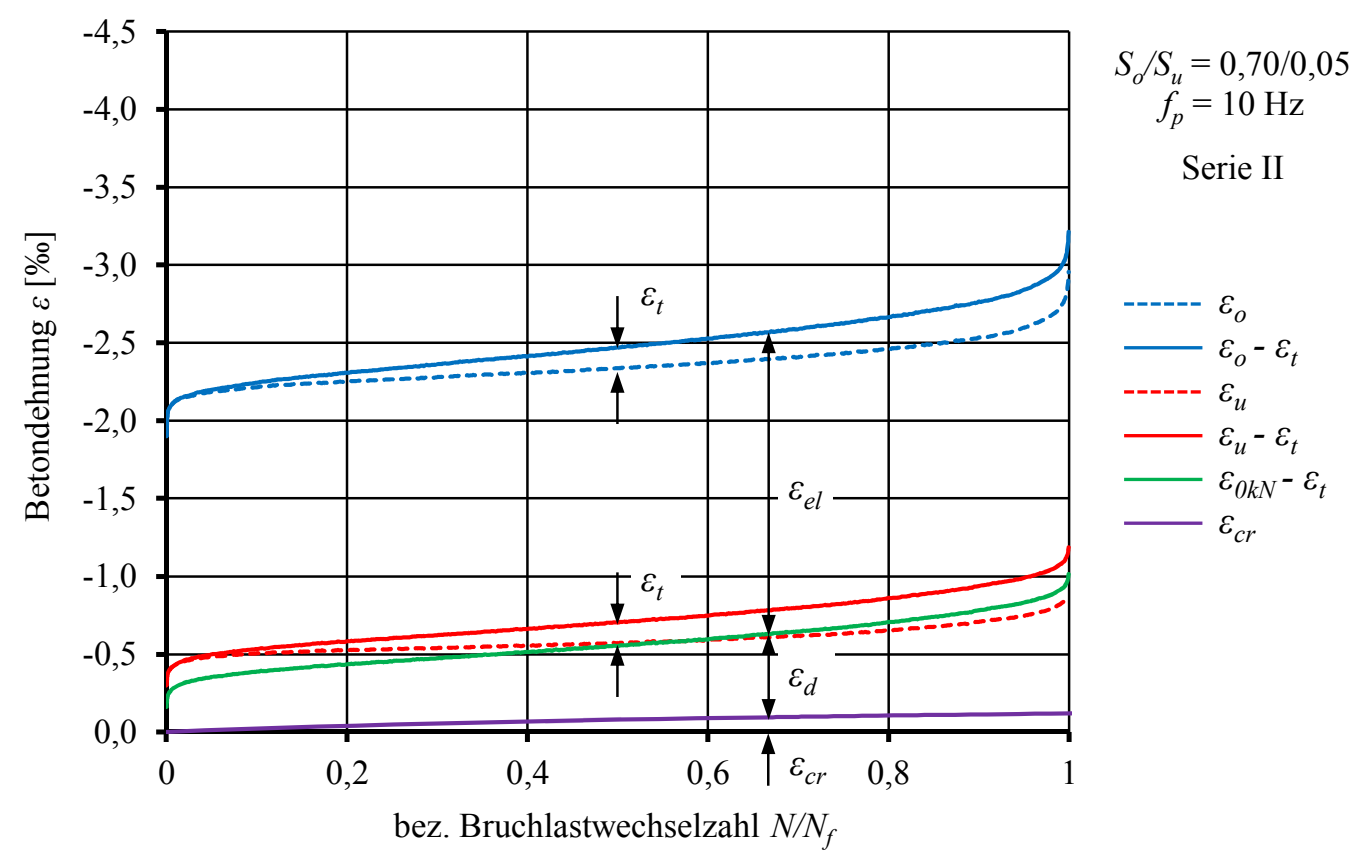

Abbildung A. 4.4: Gemittelte Dehnungsanteile in Abhängigkeit von der bezogenen Bruchlastwechselzahl Serie II, $S_{o}=0,70 / S_{u}=0,05 / f_{p}=10 \mathrm{~Hz}$ 
$S_{o}=0,70 / S_{u}=0,05 / f_{p}=1 \mathrm{~Hz}-$ Serie I

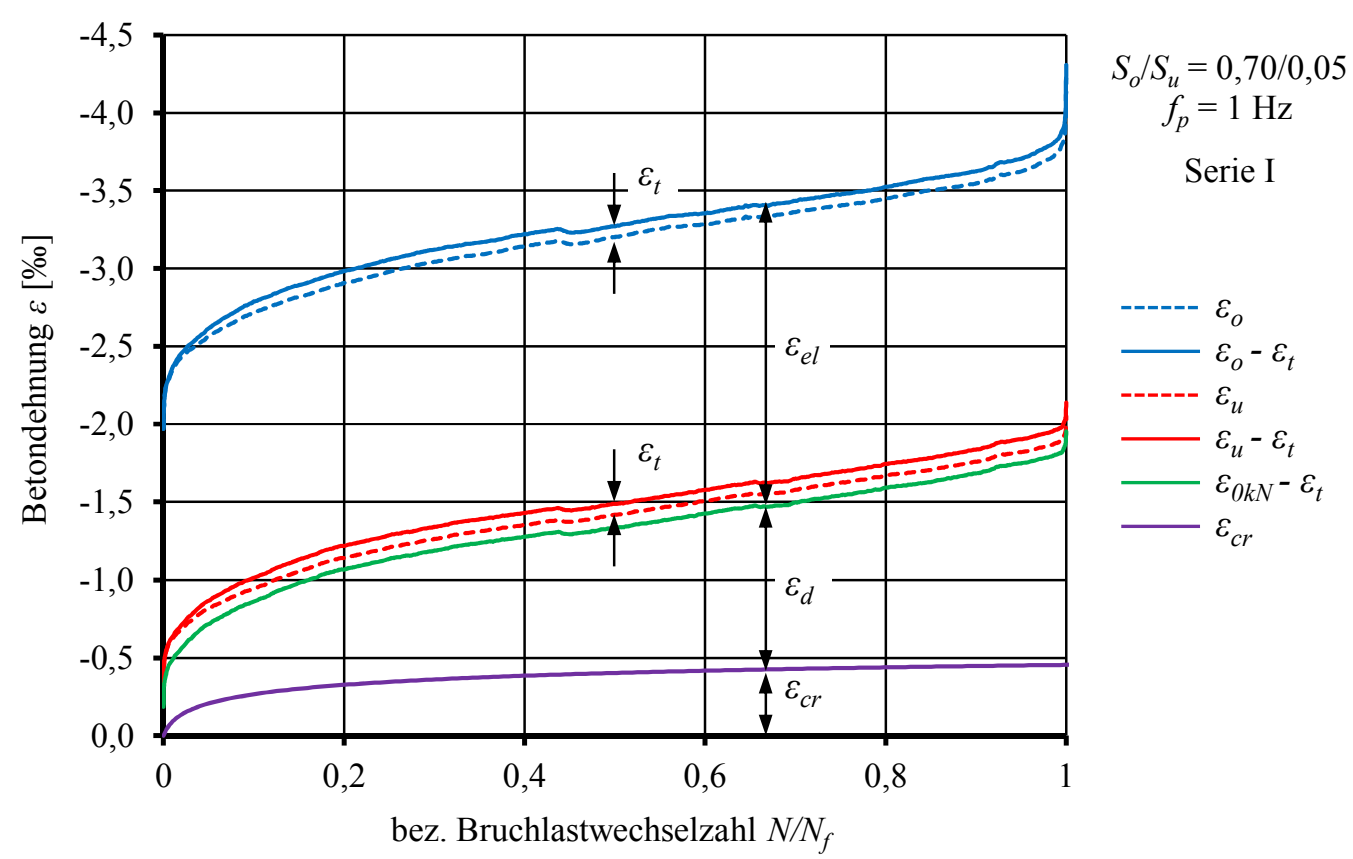

Abbildung A. 4.5: Gemittelte Dehnungsanteile in Abhängigkeit von der bezogenen Bruchlastwechselzahl Serie I, $S_{o}=0,70 / S_{u}=0,05 / f_{p}=1 \mathrm{~Hz}$ 
$S_{o}=0,60 / S_{u}=0,05 / f_{p}=10 \mathrm{~Hz}-$ Serie I

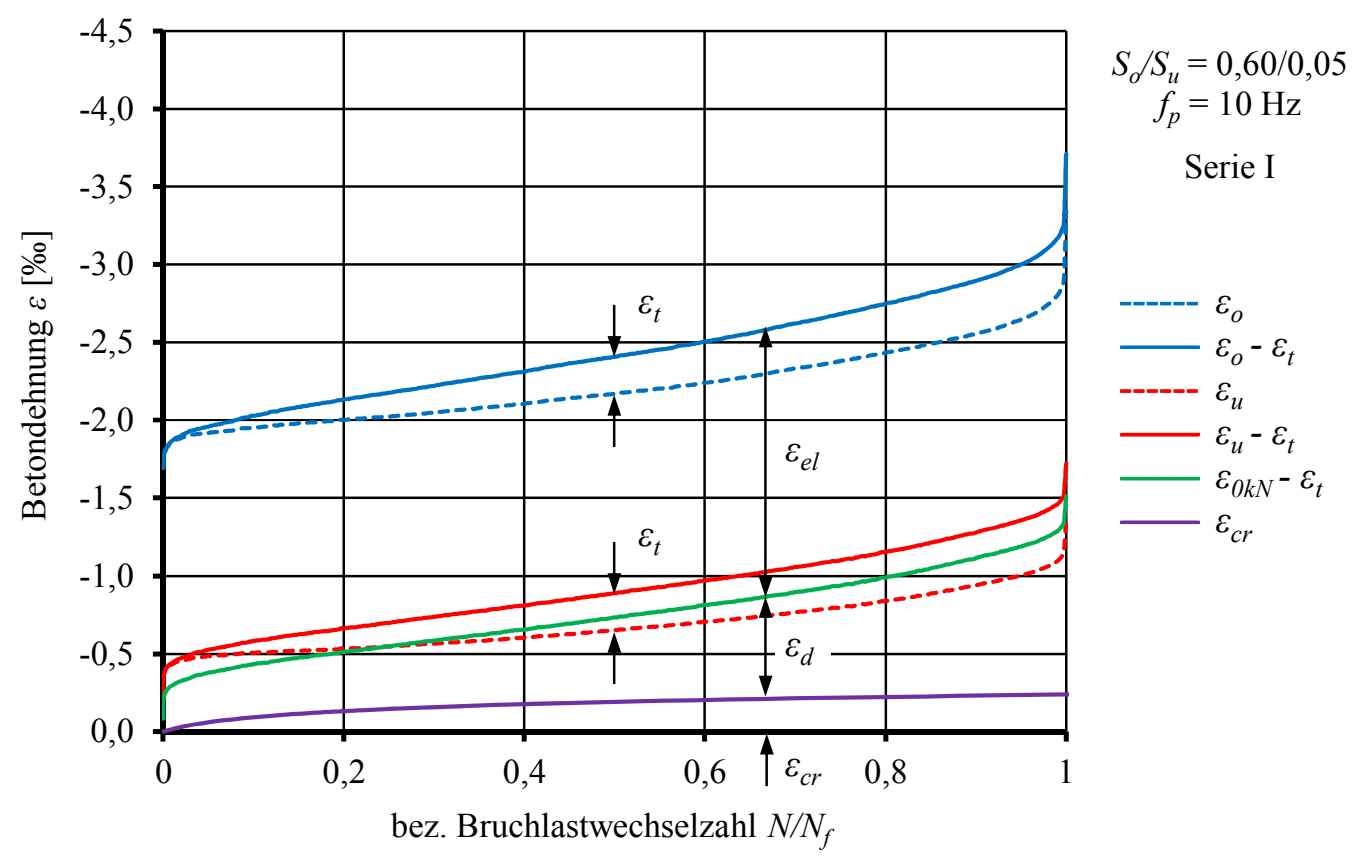

Abbildung A. 4.6: Gemittelte Dehnungsanteile in Abhängigkeit von der bezogenen Bruchlastwechselzahl Serie I, $S_{o}=0,60 / S_{u}=0,05 / f_{p}=10 \mathrm{~Hz}$

$S_{o}=0,60 / S_{u}=0,05 / f_{p}=10 \mathrm{~Hz}-$ Serie II

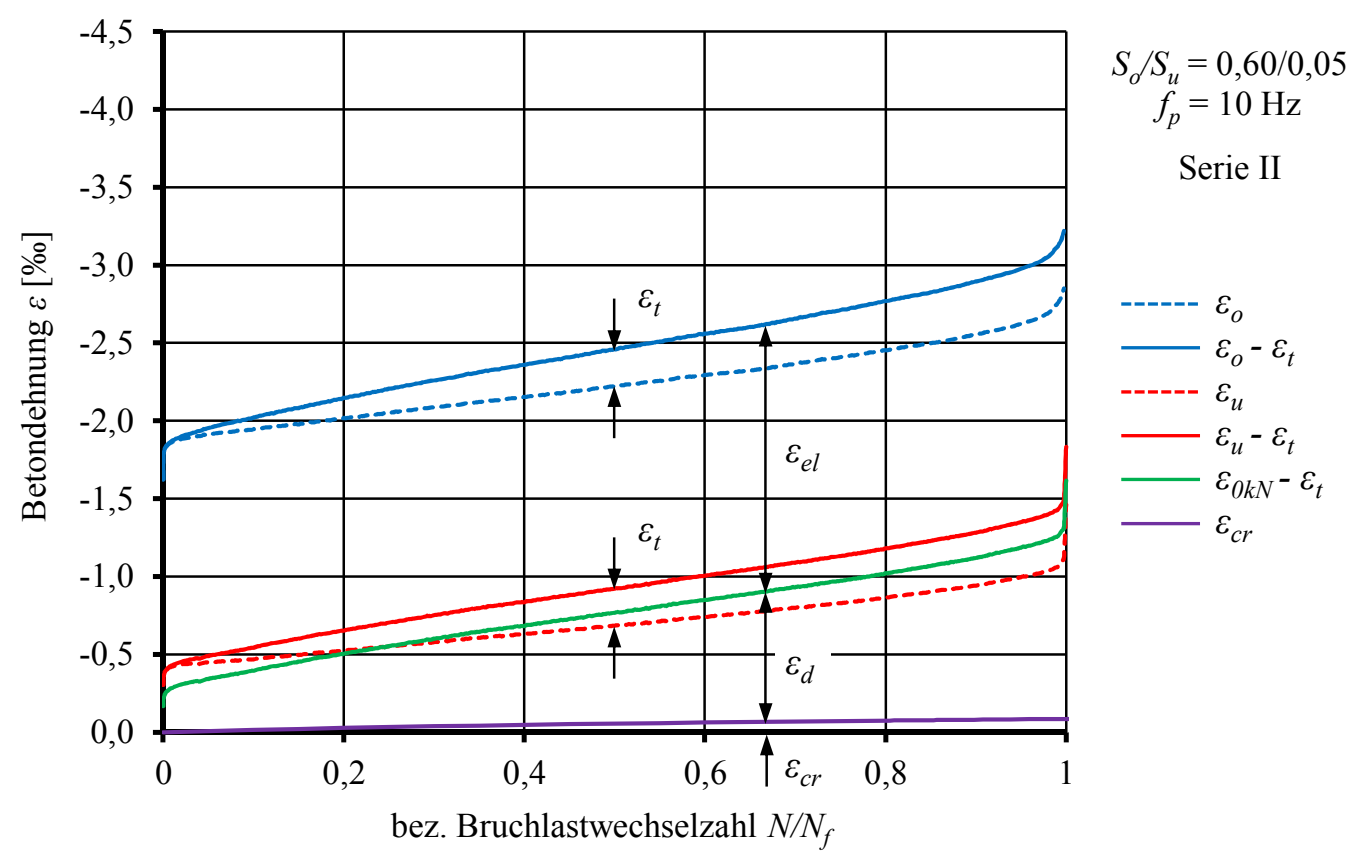

Abbildung A. 4.7: Gemittelte Dehnungsanteile in Abhängigkeit von der bezogenen Bruchlastwechselzahl Serie II, $S_{o}=0,60 / S_{u}=0,05 / f_{p}=10 \mathrm{~Hz}$ 


\section{A.5 Handlungsanweisung zur Bestimmung der Dehnungsanteile von ermüdungsbeanspruchten Betonproben}

Für die Separierung der elastischen, thermischen, viskosen und schädigungsinduzierten Dehnungen bei einem ermüdungsbeanspruchten Betonprobekörper ist ein spezielles, experimentelles Vorgehen erforderlich, welches im Folgenden zusammenfassend geschildert wird.

Während des Ermüdungsversuchs sind die Verformungen des Betonprobekörpers und die aufgebrachte Prüflast kontinuierlich aufzuzeichnen. Die Verformungen sind an drei Messstellen $\mathrm{zu}$ erfassen, die um $120^{\circ}$ zueinander versetzt in Umfangsrichtung um den Probekörper herum angeordnet sind, vgl. Abbildung A. 5.1 (rechts). Den Messwerten kann der elastische Dehnungsanteil zwischen Oberspannungsniveau und Unterspannungsniveau direkt entnommen werden; der Dehnungsanteil zwischen Unterspannungsniveau und Entlastungsniveau ist rechnerisch zu bestimmen, beispielsweise anhand des Sekantenmoduls gemäß Abschnitt 6.1. Der Sekantenmodul errechnet sich entsprechend Gleichung 2.29 aus der Prüflast und den Probekörperverformungen zwischen Oberspannungsniveau und Unterspannungsniveau. Das Unterspannungsniveau des Ermüdungsversuchs ist möglichst niedrig zu wählen, um Ungenauigkeiten bei der rechnerischen Bestimmung des elastischen Dehnungsanteils zwischen Unterspannungsniveau und Entlastungsniveau zu begrenzen. Es empfiehlt sich ein Unterspannungsniveau von $S_{u} \leq 5 \%$ der Betondruckfestigkeit.

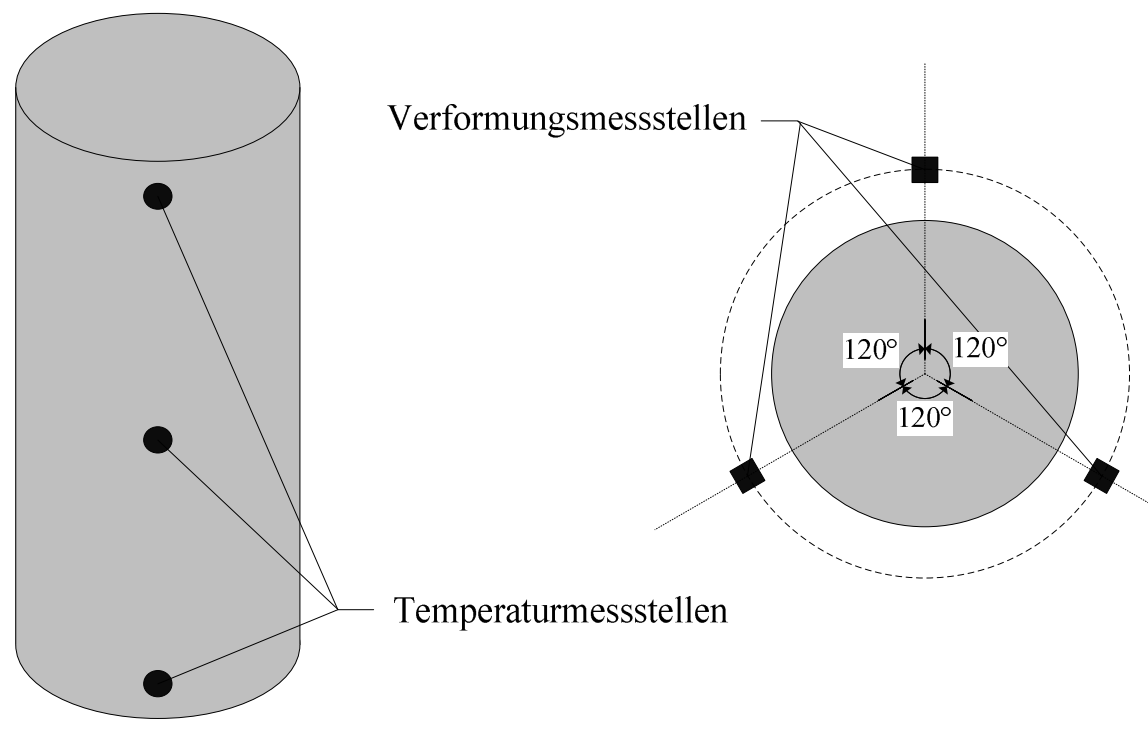

Abbildung A. 5.1: Verformungs- und Temperaturmessungen im Ermüdungsversuch

Zur Bestimmung des thermischen Dehnungsanteils ist die Temperaturänderung des Betonprobekörpers zwischen Versuchsbeginn und Versuchsende $\mathrm{zu}$ erfassen. Hierzu ist die Oberflächentemperatur an mindestens drei Stellen über die Probenhöhe aufzuzeichnen, vgl. Abbildung A. 5.1 (links). Anhand dieser Messwerte sind das Temperaturprofil des Probekörpers und eine mittlere Probekörpererwärmung entsprechend Abbildung 6.4 bzw. Gleichung 6.1 abzuschätzen. Durch weitere Messstellen kann die Abschätzung verbessert werden. Aus der 
mittleren Probekörpererwärmung kann der thermische Dehnungsanteil mithilfe des Wärmeausdehnungskoeffizienten des Betons entsprechend Gleichung 6.2 berechnet werden. Der Wärmeausdehnungskoeffizient ist abzuschätzen oder experimentell zu bestimmen.

Für die Bestimmung des viskosen Verformungsanteils sind Kriechversuche durchzuführen. Das Probenalter, die Probenlagerung bis zur Prüfung, die Probenvorbereitung und die Probenform sollten den Proben der Ermüdungsversuche entsprechen. In Anlehnung an (DAfStb, 1991) können auch längere bzw. höhere Proben verwendet werden. In den Kriechversuchen sind die Kriechverformungen und die Kriechlast zu erfassen. Die Verformungsmessung kann in Anlehnung an Abbildung A. 5.1 (rechts) erfolgen. Idealerweise werden die Kriechversuche und die Ermüdungsversuche bei gleichen Umgebungsbedingungen (Temperatur, Luftfeuchte) durchgeführt. Die Kriechversuche sind auf unterschiedlichen Spannungsniveaus durchzuführen. Ein Spannungsniveau sollte im Bereich des linearen Kriechens $\left(\sigma_{c r} \leq 0,4 \cdot f_{c}\right)$ und zwei im Bereich des nichtlinearen Kriechens durchgeführt werden $\left(\sigma_{c r}=0,5 \cdot f_{c}\right.$ und $\left.\sigma_{c r}=0,6 \cdot f_{c}\right)$, um die proportionale bzw. überproportionale Beziehung zwischen Kriechspannung und Kriechdehnung zu quantifizieren. Die Kriechspannungsniveaus sollten zudem so gewählt sein, dass die zu erwartenden kriechaffinen Beanspruchungsniveaus zwischen dem geringsten und den höchsten Kriechspannungsniveau liegen. Stimmt die proportionale bzw. überproportionale Beziehung zwischen Kriechspannung und Kriechdehnung mit der Beziehung des Model Code 2010 überein, kann das kriechaffine Spannungsniveau in Abhängigkeit des sinusförmigen Beanspruchungsverlaufs entsprechend den Abbildungen 4.12 und 4.16 bestimmt werden. Andernfalls ist das kriechaffine Spannungsniveau auf Grundlage von Gleichung $4.24 \mathrm{zu}$ ermitteln. Anhand des kriechaffinen Beanspruchungsniveaus, der proportionalen bzw. überproportionalen Beziehung zwischen Kriechspannung und Kriechdehnung sowie den experimentell aufgezeichneten Kriechkurven kann der viskose Dehnungsanteil für das kriechaffine Beanspruchungsniveau interpoliert werden.

Der schädigungsinduzierte Dehnungsanteil ergibt sich aus der Differenz zwischen den experimentell gemessenen Gesamtverformungen und den elastischen, thermischen und viskosen Verformungen entsprechend Gleichung 3.5. 


\section{Beruflicher und wissenschaftlicher Werdegang}

\section{Persönliche Daten}

Name

Christoph von der Haar

Geburtsdatum

06.06 .1984

Geburtsort

Münster

\section{Beruflicher Werdegang}

seit $10 / 2016$

$06 / 2015-09 / 2016$

$03 / 2011-09 / 2016$

$08 / 2009-02 / 2011$

$2004-2009$
Stellvertretender Institutsleiter am Institut für Massivbau der

Leibniz Universität Hannover

Forschungsgruppenleiter am Institut für Massivbau der Leibniz Universität Hannover

Wissenschaftlicher Mitarbeiter am Institut für Massivbau der Leibniz Universität Hannover

Projektingenieur/-leiter bei der grbv Ingenieure im Bauwesen GmbH \& Co. KG, Hannover

Bauingenieurstudium an der Leibniz Universität Hannover, Studienschwerpunkt: Bauwerksplanung und -konstruktion, Studienabschluss: Diplomingenieur

\section{Zeitschriftenbeiträge (mit wissenschaftlicher Qualitätssicherung)}

von der Haar, C.; Marx, S.: Development of Stiffness and Ultrasonic Pulse Velocity of Fatigue Loaded Concrete. Structural Concrete 17 (2016) Heft 4. DOI: 10.1002/suco.201600007

Hümme, J.; von der Haar, C.; Lohaus, L.; Marx, S.: Fatigue Behaviour of a Normal-Strength Concrete - Number of Cycles to Failure and Strain Development. Structural Concrete 17 (2016) Heft 4. DOI: 10.1002/suco.201500139

Stümpel, M; von der Haar, C.; Marx, S.: Zum Tragverhalten von Stützen semi-integraler Brücken - Realitätsnahe Berechnung von Stützen semi-integraler Brücken im Grenzzustand der Tragfähigkeit. Beton- und Stahlbetonbau 111 (2016) Heft 8, S. 522-531.

DOI: 10.1002/best.201600023

von der Haar, C.; Marx, S.: Entwicklung von Steifigkeit und Ultraschallgeschwindigkeit dynamisch beanspruchten Betonproben. Beton- und Stahlbetonbau 111 (2016) Heft 3, S. 141 - 148. DOI: 10.1002/best.201500059 
von der Haar, C.; Marx, S.: Design Aspects of Concrete Towers for Wind Turbines. Journal of the South African Institute of Civil Engineering (SAICE) 57 (2015) Heft 4, S. 30 - 37.

DOI: $10.17159 / 2309-8775 / 2015 / v 57 n 4 a 4$

von der Haar, C.; Marx, S.; Krompholz, R.: Ultraschalluntersuchungen an statisch beanspruchten Betonproben. Beton- und Stahlbetonbau 110 (2015) Heft 11, S. 759 - 766.

DOI: 10.1002/best.201500037

von der Haar, C.; Hümme, J.; Marx, S.; Lohaus, L.: Untersuchungen zum Ermüdungsverhalten eines höherfesten Normalbetons. Beton- und Stahlbetonbau 110 (2015) Heft 10, S. 699 - 709. DOI: $10.1002 /$ best.201500034

von der Haar, C.; Marx, S.; Bolle, G.: Base-Line-gestützte Verformungsmessungen an einer semiintegralen Hohlkastenbrücke. Bautechnik 91 (2014) Heft 2, S. 107 - 113.

DOI: 10.1002/bate.201300075

Marx, S.; von der Haar, C.; Liebig, J.P.; Grünberg, J.: Bestimmung der Verkehrseinwirkung auf Brückentragwerke aus Messungen an Fahrbahnübergangskonstruktionen. Bautechnik 90 (2013) Heft 8, S. 466 - 474. DOI: 10.1002 / bate.201300015

\section{Konferenzbeiträge (mit wissenschaftlicher Qualitätssicherung)}

von der Haar, C.; Wedel, F.; Marx, S.: Numerical and Experimental Investigations of the Warming of Fatigue-Loaded Concrete. In: fib Symposium 2016, Kapstadt, Süd Afrika, 21.-23. November 2016.

von der Haar, C.; Marx, S.: Strain Development of Plain High Strength Concrete under Fatigue Loading. In: $4^{\text {th }}$ International Symposium on Ultra-High Performance Concrete and High Performance Materials (HiPerMat 2016), Kassel, Germany, 9.-11. März 2016.

von der Haar, C.; Marx, S.; Hansen, M.; Neuner, H.: Detection of Traffic Loads by Structural and Geodetic Measurements. In: $3^{\text {rd }}$ International Symposium on Life-Cycle Civil Engineering (IALCCE 2012), Wien, Österreich, 3.-6. Oktober 2012. S. 327 - 334.

von der Haar, C.; Marx, S.; Hansen, M.: Monitoring of Bridges - Detection of Traffic Loads. In: $6^{\text {th }}$ International Conference on Bridge Maintenance, Safety and Management (IABMAS 2012), Stresa, Italien, 8.-12. Juli 2012. S. 3942 - 3947.

\section{Sonstige}

von der Haar, C.; Marx, S.: Auslegung von Suction Buckets in Betonbauweise. In: Achmus, M. (Hrsg): Suction Bucket Gründungen als innovatives und montageschallreduziertes Konzept für Offshore-Windenergieanlagen. Schlussbericht zum Forschungsvorhaben WindBucket, S. $151-172$. 
Marx, S.; von der Haar, C: Stahlbeton- und Spannbetonkonstruktionen für Offshore Windenergieanlagen. In: Design und Installation für Offshore-Windenergieanlagen und Konverterplattformen, ForWind Academy \& Haus der Technik, Hannover, 3.-4. November 2014.

von der Haar, C. (2014): Design Aspects of Concrete Towers for Wind Turbines. In: International Seminar on Design of Wind Turbine Support Structures, Stellenbosch University, Stellenbosch, Süd Afrika, 3. September 2014.

Marx, S.; von der Haar, C; Schmidt, B.: Bauwerk Offshore Windenergieanlage - wie baut man das aus Beton? In: ForWind Vortragsreihe, Oldenburg, 20. Februar 2014.

Schmitt, C.; Neuner, H.; Neumann, I.; von der Haar, C.; Hansen, M.; Marx, S.: Überwachung von Brückentragwerken mit ingenieurgeodätischen Verfahren und Sensoren der Baumesstechnik. In: 3. Tagung GeoMonitoring, Hannover, 14.-15. März 2013.

Hansen, M.; von der Haar, C.; Marx, S.: Assessment of Heavy Load Vehicles without Disability of Traffic. In: $8^{\text {th }}$ Central European Congress on Concrete Engineering (CCC 2012), Plitvicer Seen, Kroatien, 4.-6. Oktober 2012. S. 169 - 174.

Hansen, M.; von der Haar, C.; Marx, S.: Aktuelle Verkehrslastermittlungen ohne Eingriff in den fließenden Verkehr. In: VSVI-Seminar Nr. 9 "Brücken- und Ingenieurbau", Vereinigung der Straßenbau- und Verkehrsingenieure e.V., Braunschweig, 7. März 2012. S. 4-1 - 4-8.

von der Haar, C.; Helfers, B.: Neubau des Mittellandkanals über die zweigleisige DB-Strecke bei Elbeu unter Aufrechterhaltung des Bahnbetriebes. In: AIV-Bericht 2011, Architekten- und Ingenieur-Verein Hannover e.V., Hannover. S. 17 - 22. 